\title{
Development and characterization of aptamer-conjugated imaging tools for diagnostic applications
}

\author{
by
}

Anna Koudrina

A thesis submitted to the Faculty of Graduate and Postdoctoral Affairs in partial fulfillment of the requirements for the degree of

Doctor of Philosophy

in

Chemistry, Specialization in Chemical and Environmental Toxicology

Carleton University

Ottawa, Ontario

(C) 2020, Anna Koudrina 


\section{Abstract}

Magnetic resonance imaging (MRI) and computed tomography (CT) are imaging modalities commonly used to assess blood flow; however, neither can label a blood clot that may either disrupt flow, causing an ischemic stroke, or have the ability to demonstrate where a cerebral hemorrhage has occurred (hemorrhagic stroke). To improve the diagnosis and treatment of blood vessel diseases, such as stroke and aneurysms, we developed fibrinogen aptamer (FA)-functionalized contrast agents, enabling the identification and labelling of blood clots. Fibrin was chosen as the target of interest as it is involved in blood clot formation and is, therefore, a major constituent of aforementioned conditions.

Since FA was originally selected to bind fibrinogen, fibrin-binding validation was required. It was hypothesized that FA would retain some binding affinity towards the polymerized form, fibrin, given that most of the structural elements of fibrinogen remain unmodified in the final form. To assess the affinity and selectivity of FA towards nonsolution-based fibrin, FA was tagged with a green emitting fluorophore and fluorescence co-localization was monitored. FA was selective, and binding was immediate upon direct interaction, accumulating to a significant amount within minutes. Solubilized fibrin was also used in a number of binding validation studies, including microscale thermophoresis, isothermal titration calorimetry, and circular dichroism. These techniques were used to calculate the apparent $\mathrm{K}_{\mathrm{d}}$, which was found to be within the acceptable range when compared to the known $\mathrm{K}_{\mathrm{d}}$ of FA to fibrinogen.

Four different FA-targeted contrast materials were produced, including gadolinium conjugates (Gd(III)-DOTA/NOTA-FA) for MRI, iodinated-FA and FA-functionalized gold nanoparticles (FA-AuNPs) for CT, and FA-functionalized gold-coated iron-oxide 
nanoparticles ( $\mathrm{FA}-\mathrm{Fe}_{3} \mathrm{O}_{4}$-AuNPs) as dual-imaging agents for MRI and CT. All formulations were tested for their ability to produce significant contrast enhancement. In each case, when treated with aptamer-targeted formulations, contrast development was specific to the location of the clot. In MRI, T1- and T2-weighted scans were performed to visualize hyperintense contrast enhancement of fibrinogen in the blood pool and fibrin clots, respectively. In CT, both iodo-FA and FA-AuNPs showed high positive contrast enhancement, confirming that targeting minimizes dilution of the contrast material to undetectable concentrations in the presence of the blood pool. FA- $\mathrm{Fe}_{3} \mathrm{O}_{4}-\mathrm{AuNPs}$ were successful in producing clot-specific hypointense contrast in MRI, allowing for delineation of clot parameters. However, CT imaging demonstrated that higher concentrations of the nanoparticles is required, and the thin gold coating is not sufficient to produce contrast enhancement.

These novel conjugates can be used to deliver contrast materials to the site of blood clot formation, improving diagnosis and monitoring of vascular diseases that involve thrombosis. In addition, aptamer targeting allows for guided accumulation of the contrast material leading to high local concentrations, which can effectively lower the required injection concentration, thereby lowering associated toxicity of these materials. 


\section{Preface}

This preface provides bibliographical details for each article included in this thesis, as well as whether the article is reproduced in whole or in part. Use of copyrighted material is likewise acknowledged here. When citing material from this thesis, please cite the article relevant to the chapter, if the chapter is based on a publication. This thesis is written in the form of an integrated thesis, as such, it is important to note that some of the content within introductions, and materials and methods sections may be repeated.

Chapter 1 consists of a brief general introduction, providing necessary background for the research conducted in this thesis, and is in part reproduced from a review paper published in ACS Omega.

Chapter 2 is a methods chapter prepared for thesis purposes only. It consists of an overview of the methodological approach used in this thesis.

Chapter 3 is based on a manuscript submitted for publication in ACS Applied Materials \& Interfaces. The manuscript was reproduced in part and edited for formatting and clarity of presentation.

Chapter 4 is based on a manuscript submitted for publication in ACS Bioconjugate Chemistry. The manuscript was reproduced in part and edited for formatting and clarity of presentation.

Chapter 5 is based on a manuscript submitted for publication in ACS Nanoscale Advances. The manuscript reproduced in part and edited for formatting and clarity of presentation. 
Chapter 6 provides a summary and conclusions of the research topic, as well as offers a critical analysis of the currently understood limitations and suggests future directions for this research. This chapter was produced for thesis purposes only.

Pursuant to the Integrated Thesis policy of Carleton University, the "supervisor" (Maria C. DeRosa) and the "student" (Anna Koudrina) confirm that the student was fully involved in setting up and conducting the research, obtaining data and analyzing results, as well as preparing and writing the material presented in the co-authored article(s) integrated in the thesis. Additionally, the supervisor confirms the information provided by the student in this preface. 


\section{Acknowledgements}

First and foremost, I would like to thank my supervisor, Dr. Maria DeRosa. Her confidence in my potential never wavered, while her optimism and enthusiasm towards research inspired me to push myself to work harder and be a better researcher and person. I cannot begin to describe how much I have learned from her, and even as I write this, I am overwhelmed with emotion. I would also like to thank Dr. Eve Tsai at The Ottawa Hospital for her support and everything she has done throughout this project. To Dr. Greg Cron, thank you for spending countless hours exchanging ideas for optimization of our MRI imaging and for the wonderful chats that filled the time during the scans. To everyone else who has contributed to this work - Dr. Erin McConnell, Dr. Suzan Chen, Dr. Peter Kan, Dr. Robert Garcia, Jon O'Brien, Joey Zurakowski, and Celine Chartrand - none of this would have been possible without you. I would also like to thank the DeRosa lab members (current and past) for the endless laughter, and support (both when things worked out and when they did not). Finally, thank you to the whole Chemistry Department - your support throughout my graduate degree means the world.

Of course, I would not be where I am if it were not for my mother, who abandoned her life in Russia and brought me to Canada to give me the best opportunity for growth. To my whole family, thank you for believing in me and checking in with me to make sure I was happy and healthy. To all of my wonderful friends - thank you for listening to my stories and rants. I would also like to thank my boyfriend, who supported me through the toughest times by bringing me Reese's pieces and telling me that I've got this.

Finally, I would like to thank my cats - even though both of you could not be here, I'm sure you were my guardian angels to the end! 


\section{Table of Contents}

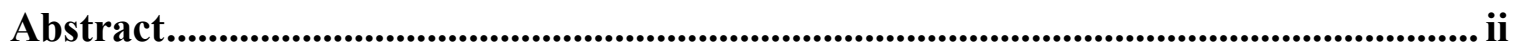

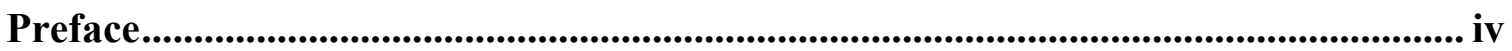

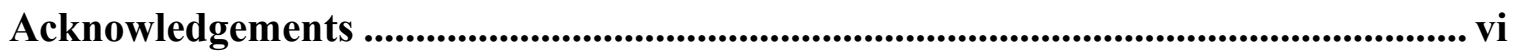

Table of Contents ....................................................................................................................... vii

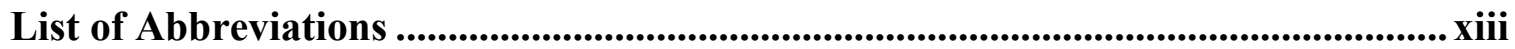

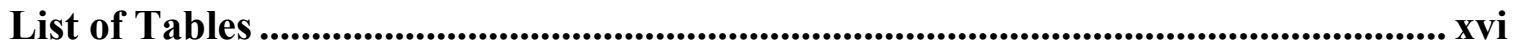

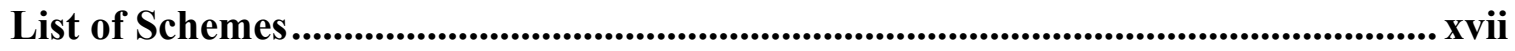

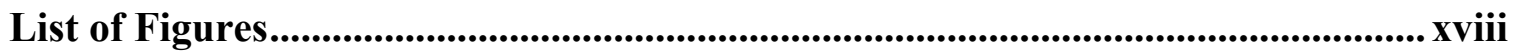

Chapter 1: An introduction to aptamers, their selection through Systematic

Evolution of Ligands by Exponential enrichment (SELEX), and a comprehensive

review of their use in MRI and CT imaging................................................................ 28

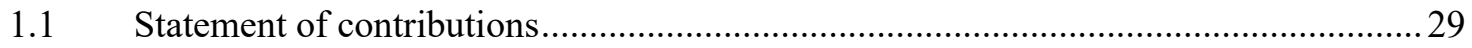

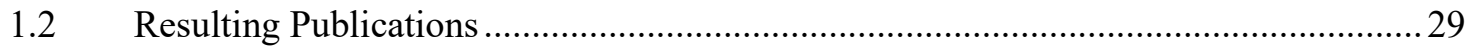

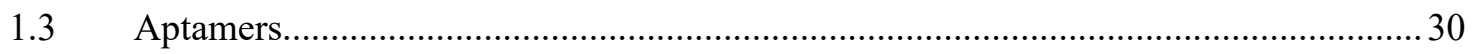

1.3.1 Systematic Evolution of Ligands by EXponential enrichment (SELEX) ................. 32

1.3.2 Considerations for the translation of aptamers to in vivo applications...................... 34

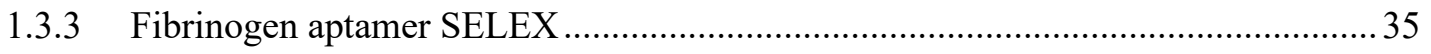

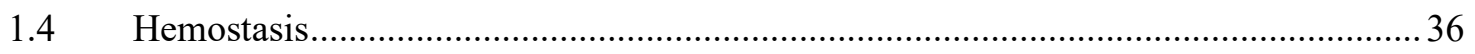

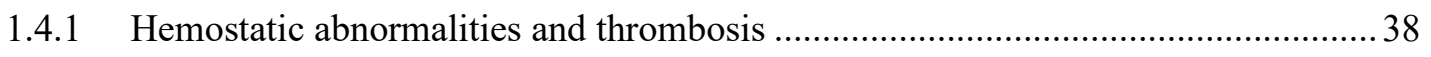

1.4.1.1 Blood clots in hemorrhagic and ischemic strokes ...................................... 40

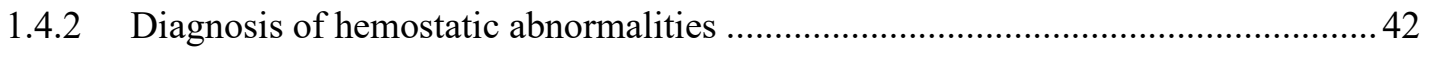

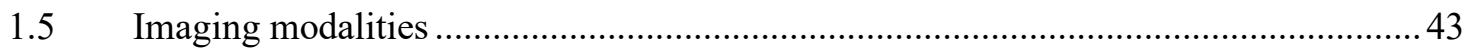

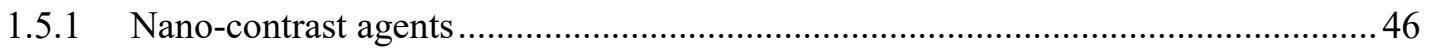




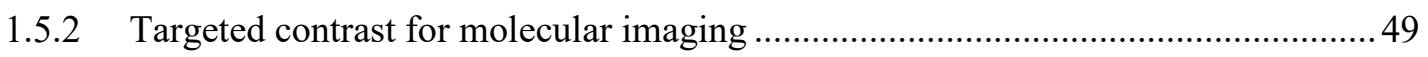

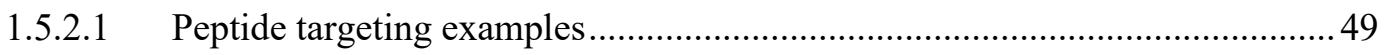

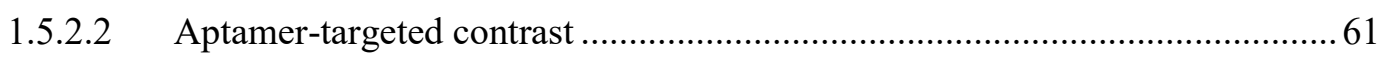

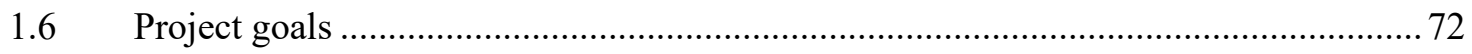

Chapter 2: Analysis Techniques .......................................................................................... 75

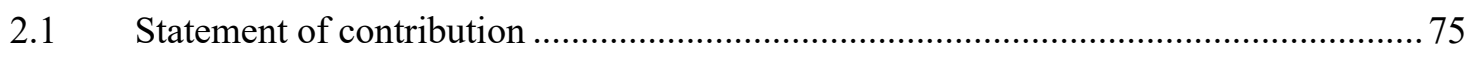

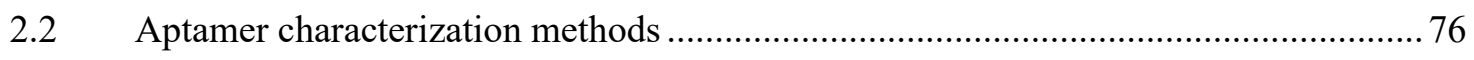

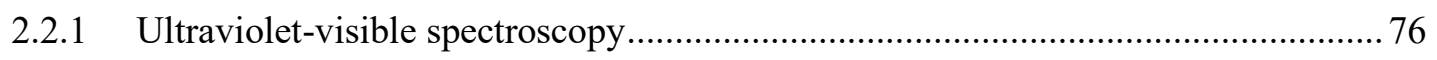

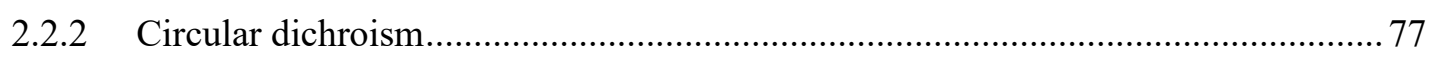

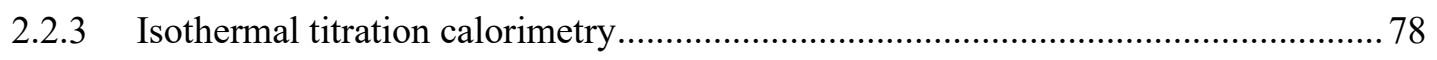

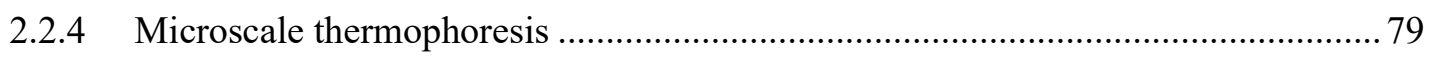

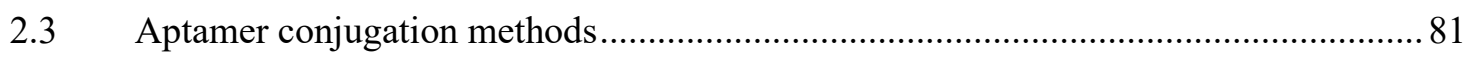

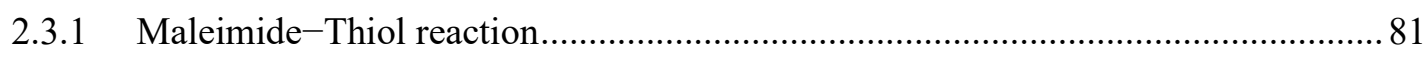

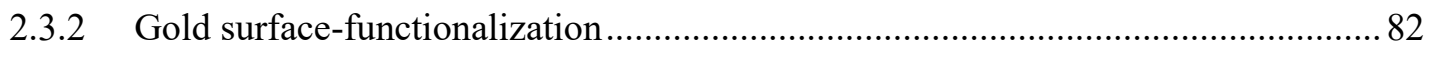

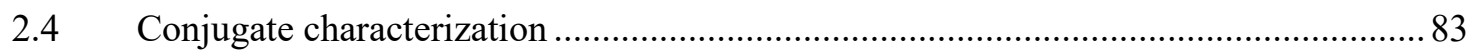

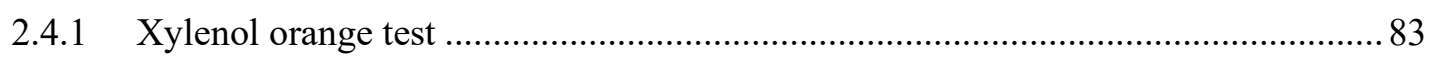

2.4.2 Transmission electron microscopy and energy-dispersive X-ray spectroscopy....... 84

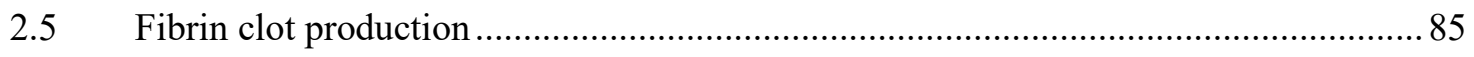

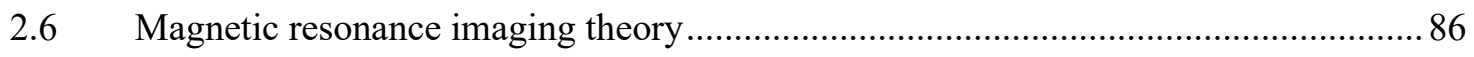

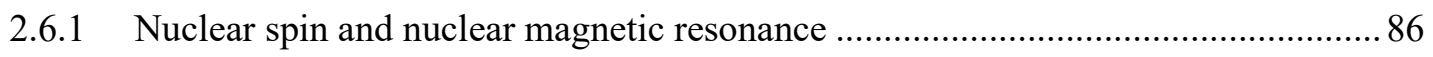

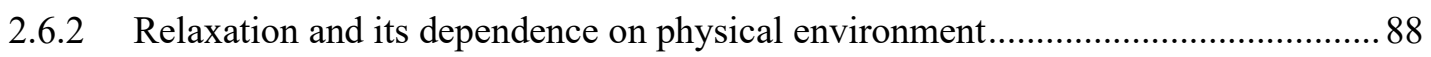

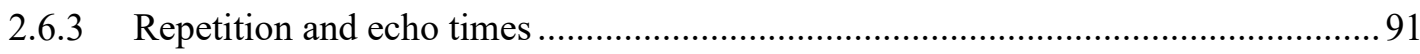

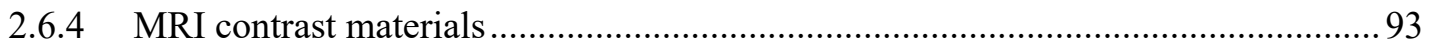

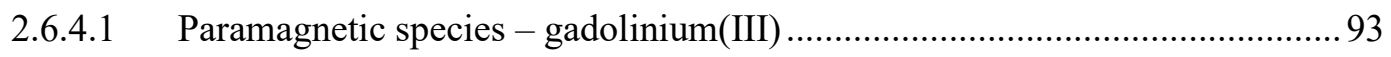

2.6.4.2 Superparamagnetic substances - iron oxide nanoparticles............................96

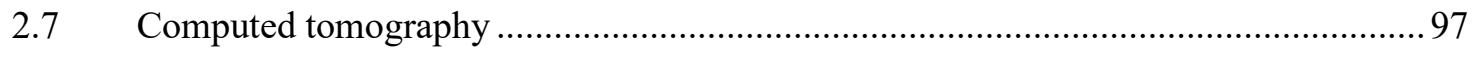




\section{Chapter 3: Developing aptamer-conjugated gadolinium contrast media to target} fibrin clots in magnetic resonance imaging ......................................................... 101

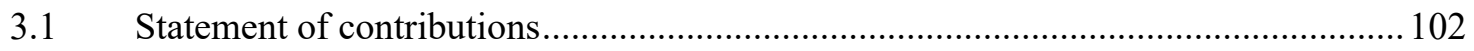

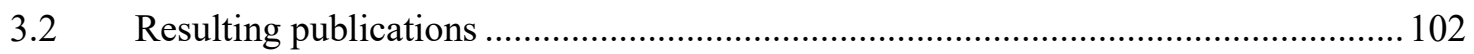

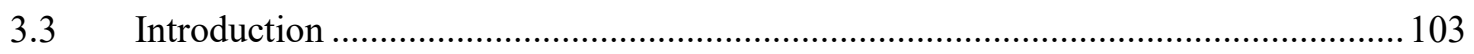

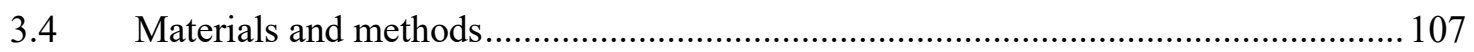

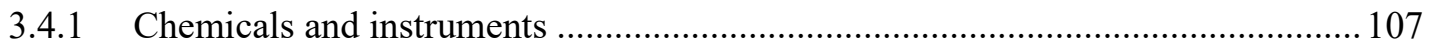

3.4.2 Preparation of Gd-DOTA/NOTA-FA to be used as an MRI probe ........................ 109

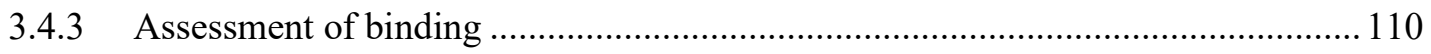

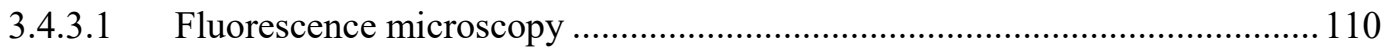

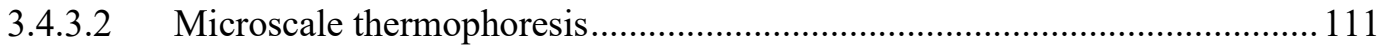

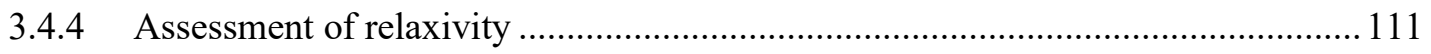

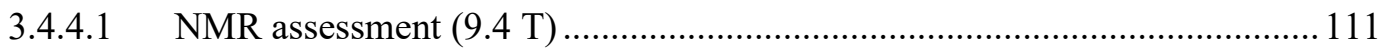

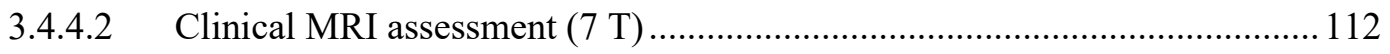

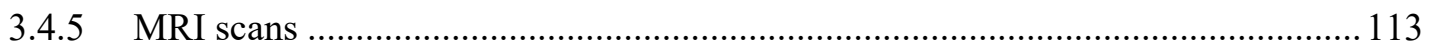

3.4.5.1 Relaxivity measurements in the presence of non-solution-based target........ 113

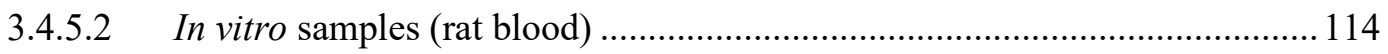

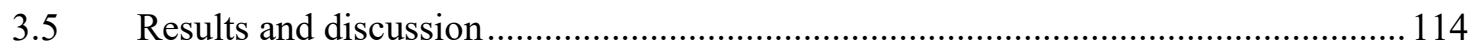

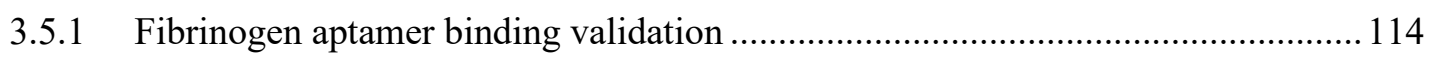

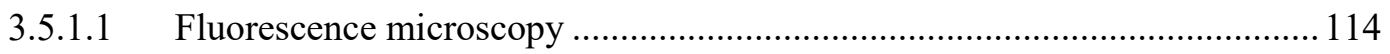

3.5.2 Synthesis and assessment of fibrinogen aptamer contrast conjugates.................... 119

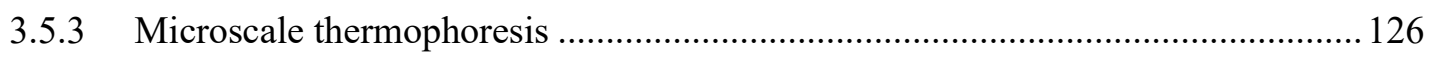

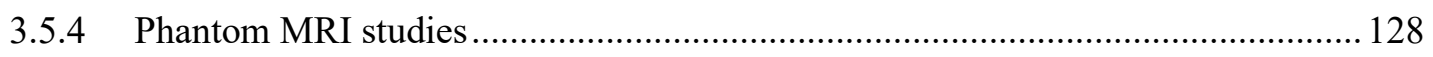

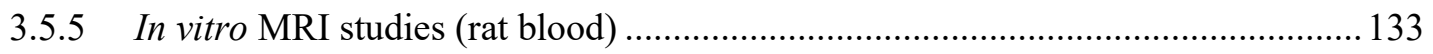




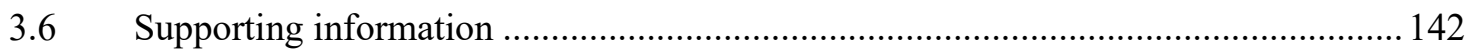

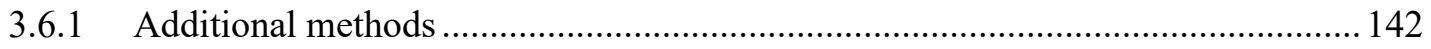

3.6.1.1 Assessment of binding kinetics and minimers ....................................... 142

3.6.1.2 Phantom MRI optimization ................................................................. 143

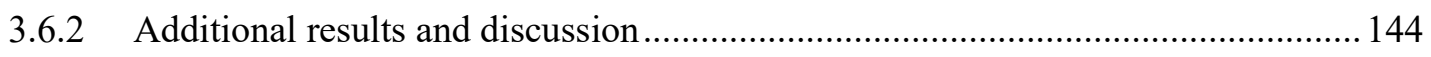

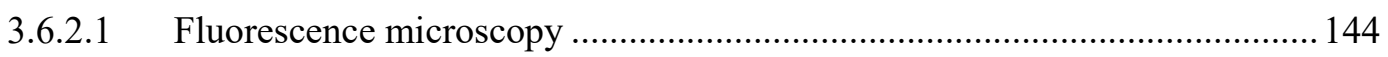

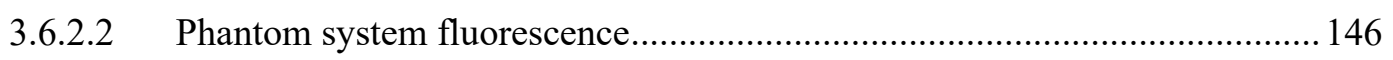

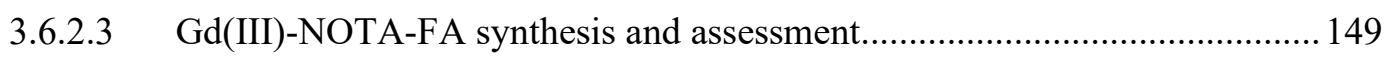

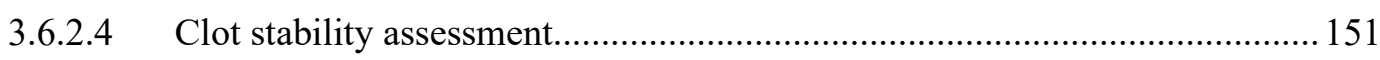

\section{Chapter 4: Assessment of aptamer-targeted contrast agents for monitoring of blood clots in computed tomography imaging ......................................................................... 153}

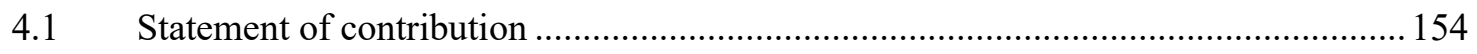

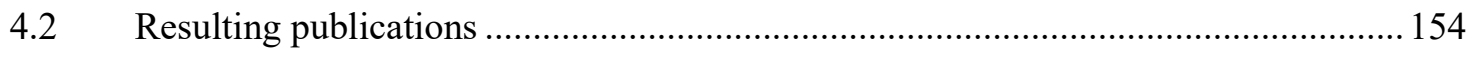

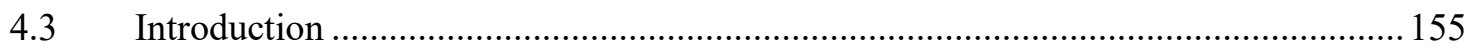

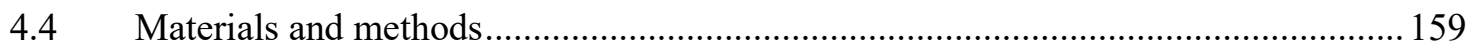

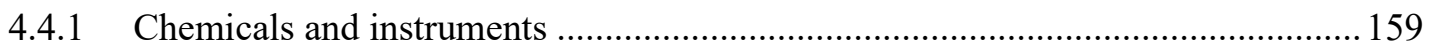

4.4.2 Preparation of aptamer-conjugated contrast materials ........................................ 161

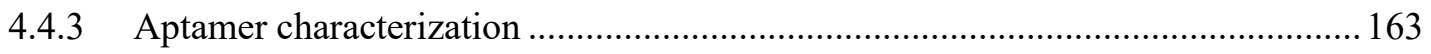

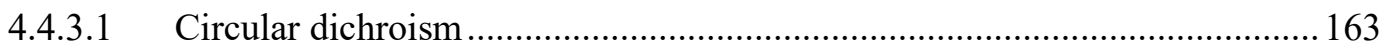

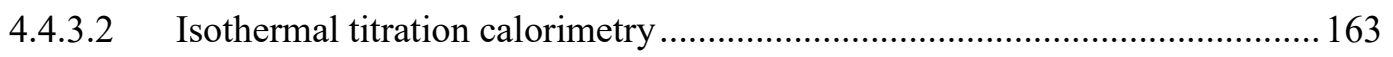

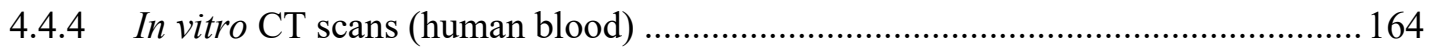

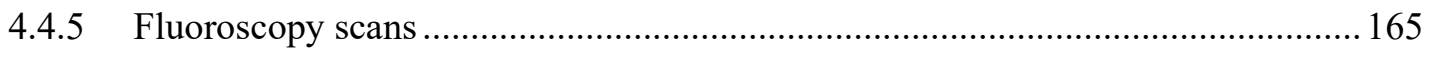

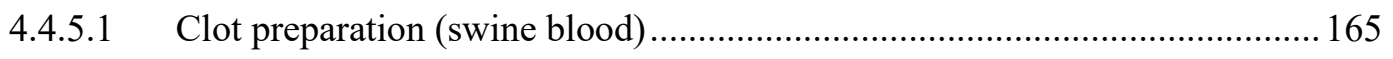

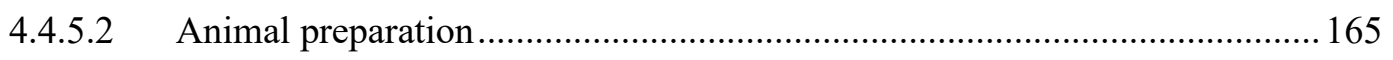

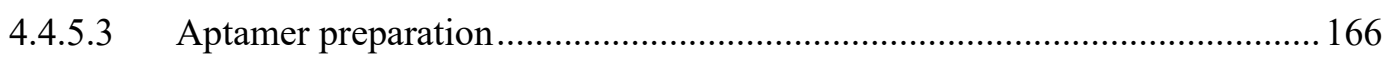

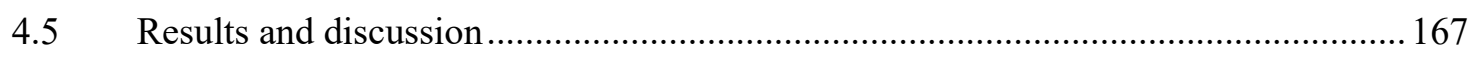


4.5.1 Preparation of aptamer-guided CT contrast materials........................................ 167

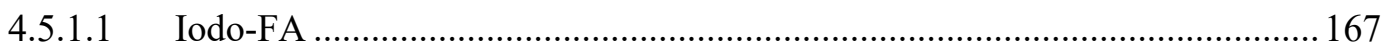

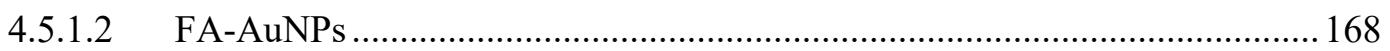

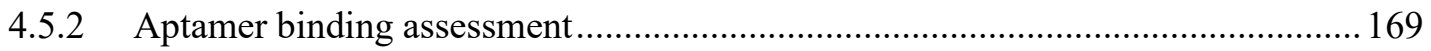

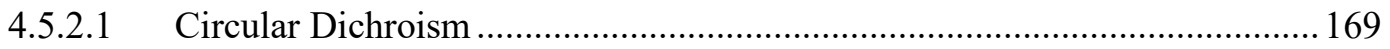

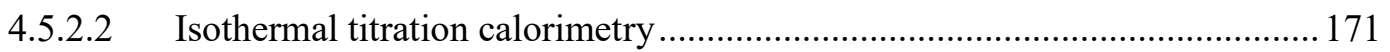

4.5.3 Assessment of CT contrast enhancement capabilities......................................... 172

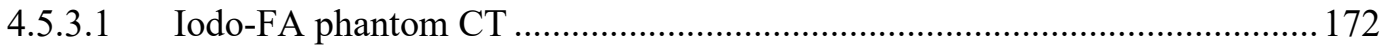

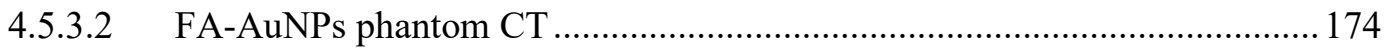

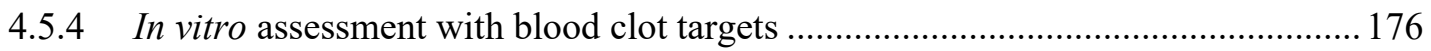

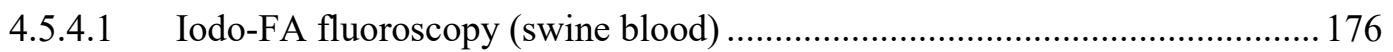

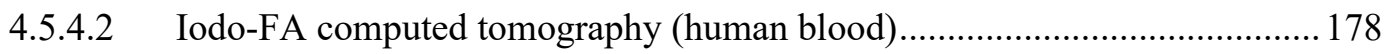

4.5.4.3 FA-AuNP computed tomography (human blood) .................................... 181

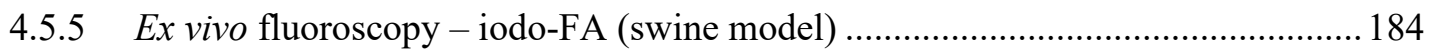

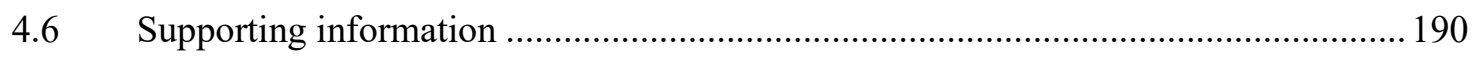

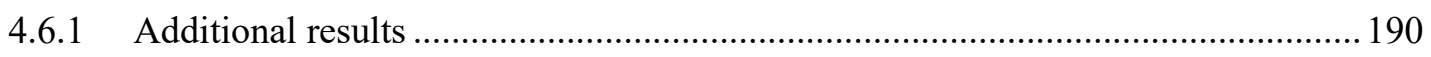

\section{Chapter 5: Aptamer functionalized gold-coated iron-oxide nanoparticles as dual}

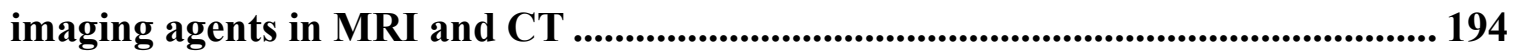

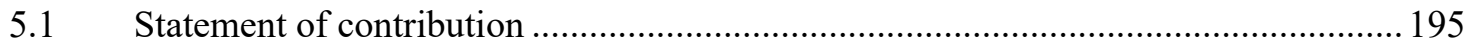

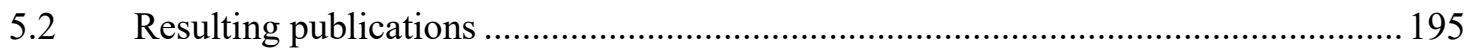

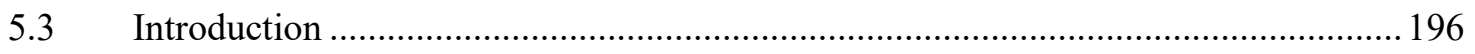

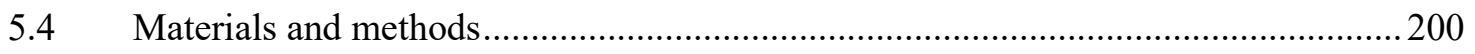

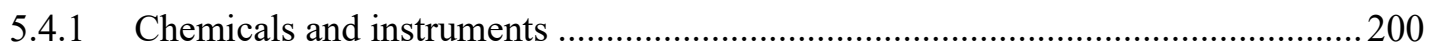

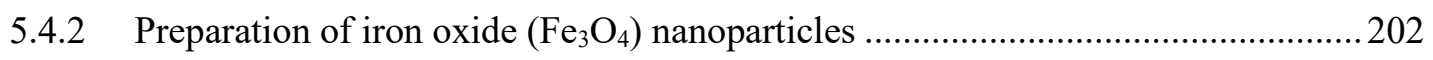

5.4.3 Direct coating of $\mathrm{Fe}_{3} \mathrm{O}_{4}$ nanoparticles to produce $\mathrm{Fe}_{3} \mathrm{O}_{4}$-AuNPs .......................... 202

5.4.4 Sodium citrate ligand exchange and conjugation with aptamer........................... 203 


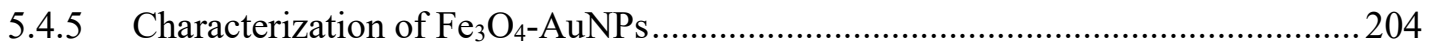

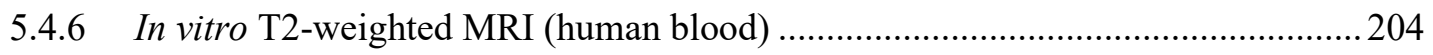

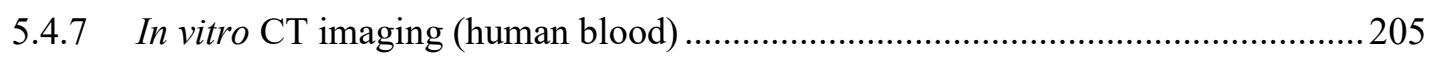

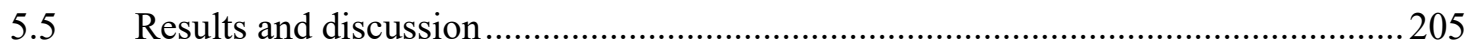

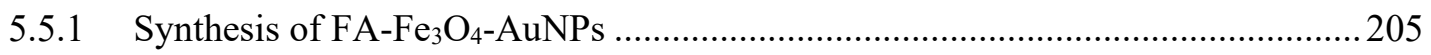

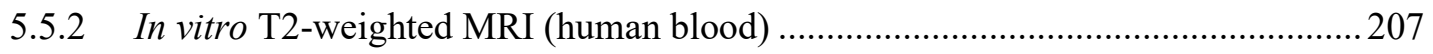

5.5.3 Phantom and in vitro CT imaging (human blood) ............................................211

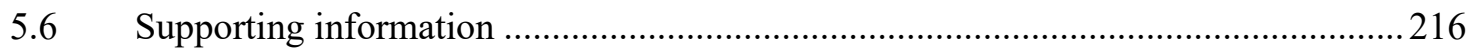

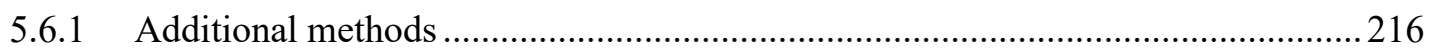

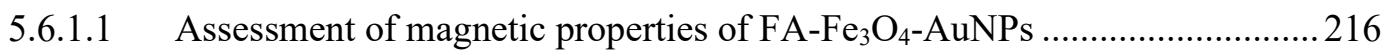

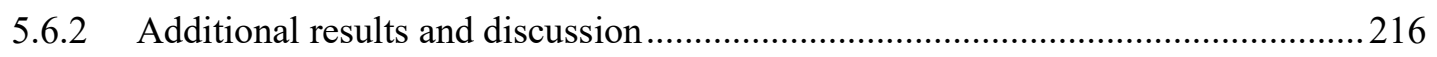

Chapter 6: Conclusions and future directions ...................................................... 224

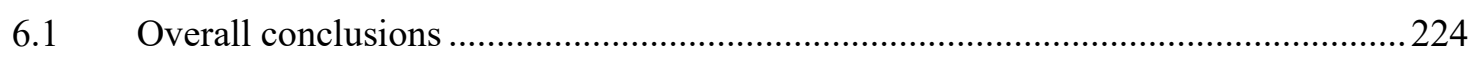

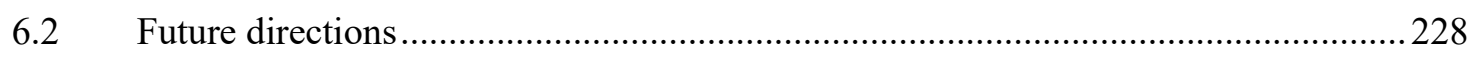

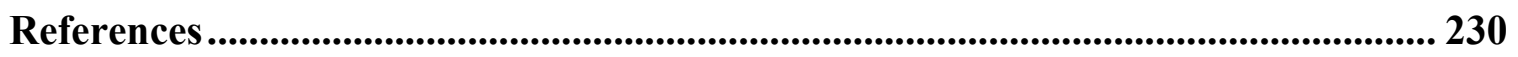




\section{List of Abbreviations}

6-FAM: $\quad$ 6-carboxyfluorescein phosphoramidite

A: $\quad$ adenine

ANOVA: analysis of variance

API: $\quad$ average pixel intensity

apt: $\quad$ aptamer

AuNPs: $\quad$ gold nanoparticles

BBB: blood-brain barrier

B-TTP: $\quad$ boronic-acid modified thymidine-5'-triphosphate

C: $\quad$ cytosine

CA: contrast agent

CCA: $\quad$ common carotid artery

CD: $\quad$ circular dichroism

CSN: $\quad$ core-shell nanoparticles

CT: $\quad$ computed tomography

DTT: dithothreitol

$\mathrm{diH}_{2} \mathrm{O}: \quad$ deionized water

DNA: deoxyribonucleic acid

DOTA: $\quad$ 1,4,7,10-tetraazacyclododecane-1,4,7,10-tetraacetic acid

DTPA: diethylenetriaminepentaacetic acid

ECA: external carotid artery

EDS: $\quad$ energy-dispersive X-ray spectroscopy

EPR: enhanced permeability and retention 
ESI-MS: electrospray ionization mass spectrometry

FA: fibrinogen aptamer

FB139: fumonisin B1 aptamer

FpA: fibrinopeptide A

FpB: fibrinopeptide B

G: guanine

Gd(III): $\quad$ gadolinium(III) ion

HU: $\quad$ Hounsfield Unit

IR: $\quad$ Infrared

ICA: internal carotid artery

ITC: $\quad$ isothermal titration calorimetry

i.v.: $\quad$ intravenous

$\mathrm{K}_{\mathrm{d}}: \quad$ dissociation constant

kVp: $\quad$ kilovoltage peak

MHz: megahertz

MIP: molecularly imprinted polymer

MRI: magnetic resonance imaging

MS: $\quad$ mass spectrometry

MST: microscale thermophoresis

mRNA: messenger ribonucleic acid

NMR: nuclear magnetic resonance

NOTA: 1,4,7-triazacyclononane-1,4,7-triacetic acid

oligo: oligonucleotide 


$\begin{array}{ll}\text { PBS: } & \text { phosphate buffered saline } \\ \text { PCR: } & \text { polymerase chain reaction } \\ \text { PEG: } & \text { polyethylene glycol } \\ \text { PET: } & \text { positron emission tomography } \\ \text { RBC: } & \text { red blood cells } \\ \text { RF: } & \text { radiofrequency } \\ \text { RNA: } & \text { ribonucleic acid } \\ \text { SAH: } & \text { subarachnoid hemorrhage } \\ \text { SAM: } & \text { self-assembled monolayers } \\ \text { SELEX: } & \text { systematic evolution of ligands by exponential enrichment } \\ \text { SPION: } & \text { superparamagnetic iron oxide nanoparticle } \\ \text { UV-Vis: } & \text { ultraviolet-visible spectroscopy } \\ \text { TEM: } & \text { single-photon emission computed tomography } \\ \text { T: } & \text { thymine } \\ \text { TE: } & \text { echo time } \\ \text { TREmission electron microscopy } & \end{array}$




\section{List of Tables}

Table 1.1: Comparison of characteristics of aptamers, antibodies, and MIPs.

Table 1.2: Summary of acquired and hereditary risk factors for primary and secondary hypercoagulable states. ${ }^{35,36}$ 40

Table 1.3: Summary of recently developed peptide-targeted contrast agents for applications in MRI, CT, and dual-imaging of various molecular markers of disease. 50

Table 1.4: Summary of the recently developed aptamer-targeted contrast agents for applications in MR and CT imaging of various molecular markers of disease. 63

Table 3.1: DNA aptamer sequences and their associated abbreviations 108

Table 3.2: Aptamer abbreviations and detailed component description. 109

Table 3.3: Average pixel intensities of each in vitro sample in T1- and T2-weighted scans, indicating significant difference between one or more treatments 137 Table S3.1: Xylenol orange test absorbance values of DOTA and NOTA complexes, with determination of concentration of free $\mathrm{Gd}^{3+}$ and loading efficiency, using $\mathrm{y}=0.0805 \mathrm{x}+$ 0.3897

Table 4.1: DNA aptamer sequences, their modifications, and associated abbreviations. Iodinated cytosine bases are denoted with * 161

Table S4.1: One-side total saturation fit results in calculating apparent dissociation constant for FA binding fibrin. 192

Table 5.1: Average contrast of blood clots associated with each treatment after a 45-minute incubation, measured in T2-weighted MRI. 210 Table 6.1: Summary of novel conjugates produced in this research, as well as their strengths and weaknesses identified at this time. 226 


\section{List of Schemes}

Scheme S3.1: Reaction scheme outlining the cleavage of the thiol-modifier C6 S-S and subsequent conjugation to maleimido-monoamide-NOTA to produce FA-NOTA conjugate. ${ }^{109}$

Scheme S4.1: Reaction scheme outlining the cleavage of the thiol-modifier C6 S-S and subsequent conjugation to the surface of AuNPs to produce FA-AuNPs.

Scheme S5.1: Reaction scheme outlining the cleavage of the disulfide bond to liberate a free thiol group and its subsequent conjugation to the surface of $\mathrm{Fe}_{3} \mathrm{O}_{4}$-AuNPs, using the

gold shell 


\section{List of Figures}

Figure 1.1: Aptamer selection through Systematic Evolution of Ligands by EXponential Enrichment (SELEX). First, a large randomized DNA pool is introduced to the target of interest. Bound sequences are partitioned from those that exhibit weak or no binding. Binders are amplified through PCR and the enriched library is reintroduced to the target. This is repeated for a number of subsequent rounds to select for sequences with the highest binding affinity and selectivity. Figure created in BioRender. 34

Figure 1.2: Visual representation of secondary hemostasis, where fibrin meshwork is formed through thrombin-catalyzed polymerization of fibrinogen upon blood vessel damage. Imaged prepared in BioRender. 38

Figure 1.3: (A) Targeted contrast agents developed towards MRI and CT applications make up more than $50 \%$ of the research in the field over the last 3 years. About a third of these utilize aptamers or peptides as their targeting moiety. Figure developed based on SciFinder search output. (B) Graphic illustration of aptamer- and peptide- targeted contrast agents for MRI and CT imaging. Image made in BioRender.

Figure 1.4: (A) Illustration of the Au:Gd@GSH nanoclusters, labelled with cyclic NGR peptide, used for dual mode imaging. (B) CT contrast phantom images of cNGRAu:Gd@GSH NMs compared to iohexol. (C) MR contrast phantom images of cNGRAu:Gd@GSH NMs compared to Magnevist. (D) MR image of tumor-bearing mouse at 24h post injection of Au:Gd@GSH-NGR, with contrast highlighting the location of the tumor in the right shoulder. (E) CT image of tumor-bearing mouse $24 \mathrm{~h}$ post injection of Au:Gd@GSH-NGR, with contrast highlighting the location of the tumor in the right shoulder. Adapted with permission from Elsevier. ${ }^{85}$ 
Figure 1.5: (A) Illustration of the dendrimer system, labelled with Gd chelate, a NIR fluorophore, and the endoglin-targeting aptamer, made in BioRender. (B) Sample T1- and T2-weighted MR imaging of hepatocellular carcinoma xenografts at $1 \mathrm{hr}$ (Middle) and 24 hrs post treatment with Den-Apt1 (Right) compared to conventional agent Gd-DTPA (Left). Adapted with permission from American Chemical Society. ${ }^{91}$

Figure 2.1: (A) MST instrumental set-up, demonstrating the simultaneous induction of a temperature gradient and excitation of the fluorophore located within the capillaries, which are scanned in sequence; (B) A sample fluorescence time-trace, indicating IR laser activation and de-activation, with thermophoresis dependent depletion of fluorophores; (C) A sample binding isotherm with ligand-target complex demonstrating a higher fluorescence plateau than the unbound species. Adapted with permission from Elsevier (Open Access). ${ }^{107}$ 81

Figure 2.2: Schematic representation of the magnetic moment of a proton and its precession about $\mathrm{B}_{0}$. Image produced in BioRender. ${ }^{55,127,128}$ 87 Figure 2.3: Schematic representation of nuclear spin of hydrogen from water molecules and its utilization in MRI, which is reconstructed in final image processing. Reprinted with permission from Springer Nature. ${ }^{55}$

Figure 2.4: Paramagnetic chelators diethylenetriaminepentaacetic acid (DTPA) (Magnevist), 1,4,7,10-tetraazacyclododecane-1,4,7,10-tetraacetic acid (DOTA) (Dotarem), 1,4,7-triazacyclononane-1,4,7-triacetic acid (NOTA). ${ }^{135}$ Structures drawn in ChemDraw. 95 
Figure 2.5: Structure of a commonly used CT contrast material, iohexol, or Omnipaque (5-[N-(2,3-Dihydroxypropyl)acetamido]-2,4,6-triiodo-N,N'-bis(2,3-

dihydroxypropyl)isophthalamide). ${ }^{143}$ Structure drawn with ChemDraw. 99

Figure 3.1: (A) Schematic representation of sample preparation for fluorescence microscopy, made in BioRender; (B) fluorescence microscopy images, under blue and green channels, with excitation wavelengths of $355 \mathrm{~nm}$ and $480 \mathrm{~nm}$, respectively: ( $1^{\text {st }}$ row) fibrin incubated with PBS, $\mathrm{pH} 7.4$, (2 ${ }^{\text {nd }}$ row) fibrin incubated with 6-FAM FB139, (3 ${ }^{\text {rd }}$ row) fibrin incubated with 6-FAM FA; (C) Average ratios of green to blue fluorescence of control, 6-FAM FB139, and 6-FAM FA, measured by ImageJ Software and compared using One-way ANOVA statistical analysis $(\mathrm{F}(2,27)=189.9, \mathrm{p}<0.0001)$, Tukey post-hoc analyses revealed a significant difference between groups $\left({ }^{*} p<0.01\right)$. Significance is indicated by $*$ 117

Figure 3.2: Longitudinal relaxivity plots of Gadovist, Gd(III)-NOTA-FA, and Gd(III)NOTA-FA incubated with fibrin at 1000:1 ratios, done at 9.4 T, pH 7.4. 123

Figure 3.3: (A) T1 map MR images of over an increasing range of concentrations of Gadovist, Gd(III)-NOTA-FA, and Gd(III)-NOTA-FA + fibrin; (B) Longitudinal relaxivity plots of Gadovist, Gd(III)-NOTA-FA, and Gd(III)-NOTA-FA incubated with fibrin at 1000:1 ratios, done at $7 \mathrm{~T}, \mathrm{pH} 7.4$...... 124

Figure 3.4: (A) T2 map MR images of over an increasing range of concentrations of Gadovist, Gd(III)-NOTA-FA, and Gd(III)-NOTA-FA + fibrin; (B) Transverse relaxivity plots of Gadovist, Gd(III)-NOTA-FA, and Gd(III)-NOTA-FA incubated with fibrin at 1000:1 ratios, done at $7 \mathrm{~T}, \mathrm{pH} 7.4$ 126 
Figure 3.5: Microscale thermophoresis analysis for: (A) FA in the presence of increasing concentrations of fibrinogen, and (B) FA in the presence of increasing concentrations of fibrin. Two technical runs were performed, and the constructed S-curve was used in determination of dissociation constants. 128

Figure 3.6: (A) T2 map MR images - fibrin and collagen proteins embedded in $0.6 \%$ agarose gel to produce phantom samples and imaged in triplicates, post incubation with Gd(III)-DOTA-FA or Gd(III)-NOTA-FA in PBS, pH 7.4 for experimental samples, and PBS, pH 7.4 or Gd(III)-DOTA-FB139 for control samples for 48 hours; (B) Average relaxation rate measurements of each sample, acquired through T2-map sequence, and compared using One-way ANOVA test $(\mathrm{F}(6,14)=42.33, \mathrm{p}<0.0001$ at $\alpha=0.05)$ and $(\mathrm{F}(6,14)=27.02, \mathrm{p}<0.0001$ at $\alpha=0.05)$; Tukey post-hoc analyses revealed a significant difference between treatments $(* \mathrm{p}<0.05, * * \mathrm{p}<0.01$, and $* * * * \mathrm{p}<0.0001)$

Figure 3.7: T2-weighted MR Image of fibrin clot incubated with PBS, pH 7.4 (left) and fibrin clot incubated with Gd(III)-NOTA-FA (right), post a 48-hour incubation. Different panels represent images taken through different depths of fibrin clot. 133

Figure 3.8: (A) Schematic representation of sample preparation for in vitro MRI imaging, made in BioRender; (B) T2-weighted MR images of clotted blood in heparinized blood pool treated with Gd(III)-NOTA-FA in PBS, pH 7.4, demonstrating the development of hyperintense signal at the location of the blood clots (blue to green, with panels representing zoomed images of 3 clots before and after treatment) over a 45-minute scan; (C) T1-weighted MR images of clotted blood in heparinized blood pool, treated with Gd(III)-NOTA-FA in PBS, pH 7.4, demonstrating the development of hyperintense signal throughout the blood pool (blue to green, with panels representing 3 different areas of the 
blood pool before and after treatment) over a 45-minute scan; (D) Average contrast enhancement of each sample, at 0 mins and 45 mins, measured by ImageJ Software and compared using One-way ANOVA test $(\mathrm{F}(15,92)=104.8$, $\mathrm{p}<0.0001)$; Tukey post-hoc analyses revealed a significant difference between treatments $(p<0.01)$. Significance is denoted by letters a through $\mathrm{d}$ and represents which treatment comparisons were made. 138

Figure S3.1: (A) Fluorescence microscopy images of 6-FAM FA incubated with fibrin, under blue and green channels, with excitation wavelengths of $355 \mathrm{~nm}$ and $480 \mathrm{~nm}$, respectively: 30 second, 1 minute, 5 minute, 10 minute incubation, and 30 minute incubations; (B) Average ratios of green to blue fluorescence at different time-points, measured by ImageJ and compared using One-way ANOVA statistical analysis $F(4,23)=$ 148.0, $\mathrm{p}<0.001$ ), Tukey post-hoc analyses revealed a significant difference between groups $* * \mathrm{p}<0.01)$. Significance is indicated by**.

Figure S3.2: (A) Predicted secondary structures of FA-m1, FA-m2, FA-m3, FA-m4 folded by Mfold; (B) Fluorescence microscopy images of 6-FAM FA-m1, 6-FAM FA-m2, 6FAM FA-m3, 6-FAM FA-m4 incubated with fibrin, under blue and green channels, with excitation wavelengths of $355 \mathrm{~nm}$ and $480 \mathrm{~nm}$, respectively. 145

Figure S3.3: Fibrin protein embedded in $0.6 \%$ agarose gel under white light (A); AlphaImager images, under excitation wavelength of $302 \mathrm{~nm}$ : (B) fibrin protein control, (C) fibrin incubated with 6-FAM FA, after 24 hours, (D) fibrin incubated with 6-FAM FA, after 48 hours, (E) fibrin incubated with 6-FAM FA, after the gel was covered with $\mathrm{H}_{2} \mathrm{O}$ for 1 week to remove unbound aptamer; (F) Average fluorescence at different time-points of 6-FAM FA incubations, measured by ImageJ and compared using One-way ANOVA 
statistical analysis $(\mathrm{F}(3,8)=592.1, \mathrm{p}<0.0001)$, Tukey post-hoc analyses revealed a significant difference between groups $(* * \mathrm{p}<0.01)$. Significance is indicated by **..... 148 Figure S3.4: Spectrophotomeric determination of $\mathrm{Gd}^{3+}$ complexed by Xylenol Orange. The increase in $\mathrm{Gd}(\mathrm{III})$ concentration causes a decrease of the band intensity at $433 \mathrm{~nm}$ and a consequent increase of the band at $573 \mathrm{~nm}$. The spectra were recorded in acetic buffer solution at $\mathrm{pH} 5.8$ in the presence of $0,5,10,15,20 \mathrm{nmol}$ of $\mathrm{Gd}^{3+}$ respectively. 150 Figure S3.5: Calibration curve obtained by spectrophotometric changes of Xylenol Orange absorptions in the presence of different amounts of $\mathrm{Gd}^{3+}$. 150 Figure S3.6: Left - human blood after clotting with thrombin, in the presence of $\mathrm{CaCl}_{2}$, for 24 hours; right - human blood with FA that was injected and incubated for 30 mins, then clotted with thrombin, in the presence of $\mathrm{CaCl}_{2}$, for 24 hours. 152 Figure 4.1: Structure of 5-I-dC-CE Phosphoramidite, used in the synthesis of Iodo-FA and -FB139. Structure drawn in ChemDraw. 162

Figure 4.2: $\mathrm{CD}$ spectrum of FA in the presence of increasing concentration of fibrin protein, illustrating the change in ellipticity at $245 \mathrm{~nm}$ and $275 \mathrm{~nm}$, associated with aptamer-target binding events. 171

Figure 4.3: (A) CT contrast phantom images of Isovue (iopamidol) and iodo-FA in the same concentration range at $100 \mathrm{kVp}$; (B) Plot of concentration-dependent contrast enhancement (HU), measured with software provided with the CT scanner, for Isovue (iopamidol) and iodo-FA. 174

Figure 4.4: (A) CT contrast phantom images of Isovue (iopamidol) and FA-AuNPs, both with increasing concentrations, at $100 \mathrm{kVp}$; (B) Plot of concentration-dependent contrast 
enhancement (HU), measured with software provided with the CT scanner, for FA-AuNPs.

Figure 4.5: (A) Left - clotted pig blood prior to treatment with iodo-FA, middle - in vitro fluoroscopy images of iodo-FA and clotted blood, with iodo-FA visualizable while the clot is not, right - in vitro fluoroscopy images (positive and negative settings) of the clot after treatment with iodo-FA and incubations overnight; (B) Average contrast enhancement (based on API) associated with iodo-FA, blood clot, and observed after treatment of the clot with iodo-FA, measured with Image J software and compared using One-way ANOVA test; Tukey post-hoc analyses revealed a significant difference between treatments $(* \mathrm{p}<0.05)$ 178

Figure 4.6: (A) In vitro CT scans of blood clots treated with iodo-FA in blood, iodo-FA in PBS, as well as control treatments with iodo-FB139, Isovue (iopamidol), and PBS, all done in triplicate, at $100 \mathrm{kVp}$; (B) Average contrast enhancement (HU) associated with blood clots and the pool of every treatment, measured with CT software provided with the scanner and compared using One-way ANOVA test $(\mathrm{F}(9,50)=55.2, \mathrm{p}<0.001$ at $\alpha=0.05)$; Tukey post-hoc analyses revealed a significant difference between treatments $(* * p<0.01) \ldots .181$ Figure 4.7: (A) In vitro CT scans of blood clots treated with FA-AuNPs in blood, FAAuNPs in PBS, as well as control treatments with FB139-AuNPs, Isovue (iopamidol), and PBS, all done in triplicate, at $100 \mathrm{kVp}$; (B) Average contrast enhancement (HU) associated with blood clots and the pool of every treatment, measured with CT software provided with the scanner and compared using One-way ANOVA test $(\mathrm{F}(9,50)=93.7, \mathrm{p}<0.0001$ at $\alpha=$ 0.05); Tukey post-hoc analyses revealed a significant difference between treatments

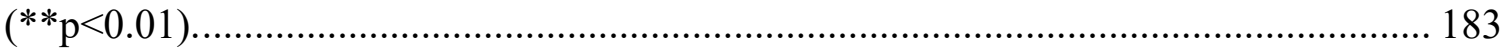


Figure 4.8: In vivo fluoroscopy scan of external carotid arteries with (left) and without (right) a large occlusion disrupting blood and contrast flow. Dashed line indicates the presence of a full occlusion in R ECA. 185

Figure 4.9: (A) Schematic representation of the workflow in preparation for ex vivo fluoroscopy assessment, made in BioRender; (B) Top - harvested external carotid arteries without (left) and with (right) a large occlusion, highlighted in red, bottom - fluoroscopy scan of external carotid arteries without (left) and with (right) a large occlusion, visualized with hyperintense contrast and highlighted in red; (C) Average contrast enhancement (API) measured with ImageJ and compared using One-way ANOVA test $(\mathrm{F}(2,12)=43.45$, $\mathrm{p}<0.0001$ at $\alpha=0.01$ ). Tukey post-hoc analyses revealed a significant difference between treatments $(* * \mathrm{p}<0.01)$ 187

Figure S4.1: Predicted secondary structure of FA (left) with $\Delta \mathrm{G}=-9.15 \mathrm{kcal} / \mathrm{mol}$ at $37^{\circ} \mathrm{C}$ and FB139 aptamer (right) with $\Delta \mathrm{G}=-4.17 \mathrm{kcal} / \mathrm{mol}$ at $37{ }^{\circ} \mathrm{C}$; 5-iodocystine bases are highlighted in green. Both sequences were folded by Mfold. ${ }^{203}$ 190

Figure S4.2: UV-Vis spectrum of lyophilized fibrin powder solubilized in $1 \mathrm{M} \mathrm{NaOH}$, assessing stability in solution form after 3 months of storage. The peak at $280 \mathrm{~nm}$ was monitored, corresponding to the absorbance of fibrinogen constituents.

Figure S4.3: CD spectrum of FB139 in the presence of increasing concentration of fibrin protein. 192

Figure S4.4: ITC thermogram for FA: (Left) heat changes measured during each injection of chemically titrated fibrin target; (Right) binding isotherm of unmodified FA with fibrin target used for calculation of apparent $\mathrm{K}_{\mathrm{d}}$ in BindWorks. 192 
Figure S4.5: ITC thermogram for iodo-FA: (Left) heat changes measured during each injection of chemically titrated fibrin target; (Right) binding isotherm of Iodo-FA with fibrin target used for calculation of apparent $K_{d}$ in BindWorks (note that $1^{\text {st }}$ injection was excluded from analysis) 193

Figure 5.1: (A) Schematic representation of sample preparation for in vitro MRI imaging, made in BioRender; (B) T2-weighted MR images of clotted blood (done in triplicate) that underwent the following treatments: $1^{\text {st }}$ row - clotted blood in a heparinized blood pool treated with $\mathrm{FA}-\mathrm{Fe}_{3} \mathrm{O}_{4}-\mathrm{AuNPs}, 2^{\text {nd }}$ row - clotted blood in a PBS pool treated with FA$\mathrm{Fe}_{3} \mathrm{O}_{4}$-AuNPs, $3^{\text {rd }}$ row - clotted blood in a heparinized blood pool treated with FB139$\mathrm{Fe}_{3} \mathrm{O}_{4}$-AuNPs (control), $4^{\text {th }}$ row - clotted blood in a heparinized blood pool treated with PBS (control); (D) Average contrast enhancement of each sample, measured by ImageJ Software and compared using One-way ANOVA test $(\mathrm{F}(3,20)=29.08, \mathrm{p}<0.0001$ at $\alpha=$ 0.05); Tukey post-hoc analyses revealed a significant difference between treatments $(* * \mathrm{p}<0.01)$ 211

Figure 5.2: (A) CT contrast phantom images of Isovue (iopamidol) and $\mathrm{FA}-\mathrm{FA}-\mathrm{Fe}_{3} \mathrm{O}_{4}-$ AuNPs, both with increasing concentrations, at $100 \mathrm{kVp}$; (B) Plot of concentrationdependent contrast enhancement (HU), measured with software provided with the CT

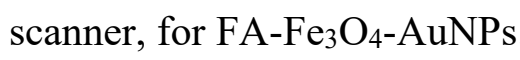

Figure S5.1: (A) Transmission electron microscopy (TEM) image with $100 \mathrm{~nm}$ scale bar and corresponding energy-dispersive X-ray spectroscopy (EDS) spectrum of small, monodispersed $\mathrm{Fe}_{3} \mathrm{O}_{4}$-core. Strong EDS peaks for both $\mathrm{Fe}$ and $\mathrm{O}$ indicate the presence of 
iron oxide. (B) Histogram demonstrating the size distribution, with average diameter of $4.95 \pm 1.27(\mathrm{n}=229)$.

Figure S5.2: (A) TEM image with $100 \mathrm{~nm}$ scale bar and corresponding EDS spectrum of the $\mathrm{Fe}_{3} \mathrm{O}_{4}$-AuNPs in phenyl ether. Strong EDS peaks for both $\mathrm{Fe}$ and $\mathrm{O}$ indicate the presence of iron oxide, while the presence of a peak for Au confirms gold-coating. (B) Histogram demonstrating the size distribution, with average diameter of $5.13 \pm 1.14$ $(n=347)$. 218

Figure S5.3: TEM image with $100 \mathrm{~nm}$ scale bar and corresponding EDS spectrum of FA$\mathrm{Fe}_{3} \mathrm{O}_{4}$-AuNPs in $\mathrm{diH}_{2} \mathrm{O}$. (B) Histogram demonstrating the size distribution, with average diameter of $5.42 \pm 0.72(\mathrm{n}=253)$. 219 Figure S5.4: UV-Vis spectra of $\mathrm{Fe}_{3} \mathrm{O}_{4}$-core, $\mathrm{Fe}_{3} \mathrm{O}_{4}-\mathrm{AuNPs}$, and $\mathrm{FA}-\mathrm{Fe}_{3} \mathrm{O}_{4}$-AuNPs in their respective buffers 220

Figure S5.5: Fibrin incubated with bare $\mathrm{Fe}_{3} \mathrm{O}_{4}$-AuNPs (left), $\mathrm{FA}_{-}-\mathrm{Fe}_{3} \mathrm{O}_{4}$-AuNPs (middle), and magnetized fibrin (with bound $\mathrm{FA}-\mathrm{Fe}_{3} \mathrm{O}_{4}$-AuNPs after 10x wash with PBS) on a rare earth magnet. Fibrin magnetization is demonstrated by accumulation of small dark powder accumulating in the middle. 221

Figure S5.6: (A) CT images of human blood clots 45-minutes after treatment with FA$\mathrm{Fe}_{3} \mathrm{O}_{4}$-AuNPs, $\mathrm{FB} 139-\mathrm{Fe}_{3} \mathrm{O}_{4}-\mathrm{AuNPs}$, Isovue, or PBS, pH 7.4, all incubations were done in triplicate; (B) Contrast, measured based on average pixel intensity using ImageJ software, associated with each treatment. 222 Figure S5.7: High-resolution T2-weighted images of blood clots that underwent the following treatments: $1^{\text {st }}$ row - clotted blood in a heparinized blood pool treated with FA$\mathrm{Fe}_{3} \mathrm{O}_{4}$-AuNPs, $2^{\text {nd }}$ row - clotted blood in a PBS pool treated with $\mathrm{FA}-\mathrm{Fe}_{3} \mathrm{O}_{4}-\mathrm{AuNPs} . .223$ 
Chapter 1: An introduction to aptamers, their selection through Systematic Evolution of Ligands by Exponential enrichment (SELEX), and a comprehensive review of their use in MRI and CT imaging 


\subsection{Statement of contributions}

The introduction chapter was written by A Koudrina and is in part based on a review, which was written and edited by A Koudrina and MC DeRosa; it contains literature up to June 2020.

\subsection{Resulting Publications}

This chapter is largely based on the following review entitled "Advances in medical imaging: aptamer- and peptide-targeted MRI and CT contrast agents" published in ACS Omega (Koudrina A, DeRosa MC, ACS Omega. 5(36):22691-22701) 


\subsection{Aptamers}

It is broadly accepted that, in living systems, deoxyribonucleic acid (DNA) and ribonucleic acid (RNA) serve an essential function in preservation, transfer, and expression of hereditary information. The Central Dogma of molecular biology, first introduced by Francis Crick in 1958, describes the sequential transfer of this genetic information from DNA to RNA, which then encodes proteins. ${ }^{1,2}$ In the context of these three biomacromolecules, proteins were classified as functional molecules responsible for a vast array of biological functions, while DNA and RNA were simply the blueprint and the intermediary, respectively. ${ }^{2}$ This remained unchallenged until the discovery of RNA enzymes (ribozymes) involved in catalysis of RNA self-cleavage or transesterification in splicing. ${ }^{3,4}$ Later, three independent groups developed an evolutionary screening process for the selection of RNA molecules that can either catalyze a reaction or bind a target molecule..$^{5-7}$

The term "aptamer" was originally introduced by Ellington and Szostak, and was derived from Latin aptus, meaning "to fit". ${ }^{8}$ Both naturally occurring and synthetic aptamers exist. Naturally occurring aptamers reside within riboswitches, which are elements of the 5'-untranslated region (UTR) of messenger RNA (mRNA) and exist in order to impart genetic regulation at mRNA level in the bacterial kingdom. ${ }^{9}$ Synthetic aptamers are short oligonucleotides (oligo) or peptides that can bind to a specific target with high affinity and selectivity. ${ }^{10}$ Both DNA and RNA aptamers can be selected from a

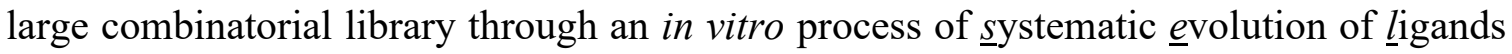
by exponential enrichment (SELEX). ${ }^{11}$ These single-stranded oligonucleotides can then assume a higher-order structure that allows for binding to occur. Aptamers offer a number 
of competitive advantages over other more established affinity ligands, such as antibodies and molecularly imprinted polymers (MIPs), as they exhibit higher stability and low immunogenicity and toxicity. ${ }^{12-14}$ Their selectivity has been shown to distinguish differences in a single functional group,${ }^{15}$ while the target range encompasses anywhere from small molecules to whole proteins and cells. ${ }^{16}$ In addition, aptamers are chemically synthesized which means they experience little batch-to-batch variation and can be easily and homogeneously modified. ${ }^{13}$ A more detailed comparison of the critical features of aptamers, antibodies, and MIPs can be found in Table 1.1. ${ }^{12,16-18}$ As a result, aptamers have been used in a number of different disciplines, including but not limited to diagnostics, therapeutics, biosensors, and others..$^{12,13,19}$ 
Table 1.1: Comparison of characteristics of aptamers, antibodies, and MIPs.

\begin{tabular}{|c|c|c|c|}
\hline & Aptamers & Antibodies & MIPs \\
\hline Average size (kDa) & $5-30$ & $150-160$ & $\mathrm{~N} / \mathrm{A}$ \\
\hline Selection & In vitro (synthetic) & $\begin{array}{c}\text { In vivo } \\
\text { (recombinant or } \\
\text { animal) }\end{array}$ & $\begin{array}{c}\text { In vitro (synthetic, } \\
\text { large scale) }\end{array}$ \\
\hline Target molecules & $\begin{array}{c}\text { From ions and } \\
\text { small molecules to } \\
\text { whole proteins and } \\
\text { cells }\end{array}$ & $\begin{array}{l}\text { Immunogenic } \\
\text { macromolecules }\end{array}$ & $\begin{array}{l}\text { Predominantly } \\
\text { small molecules }\end{array}$ \\
\hline Stability & $\begin{array}{l}\text { DNA - years at } \\
\text { room temp } \\
\text { RNA - several } \\
\text { months at }-80^{\circ} \mathrm{C} \\
\text { *in the absence of } \\
\text { nucleases }\end{array}$ & $\begin{array}{c}\text { Several weeks } \\
\text { when stored at } 4^{\circ} \mathrm{C}\end{array}$ & Years at room temp \\
\hline $\begin{array}{r}\text { Chemical } \\
\text { Modifications }\end{array}$ & $\begin{array}{l}\text { Can be readily and } \\
\text { easily modified } \\
\text { without affinity } \\
\text { loss, homogeneous } \\
\text { products }\end{array}$ & $\begin{array}{l}\text { Feasible, often } \\
\text { leads to reduced } \\
\text { activity, } \\
\text { heterogeneous } \\
\text { products }\end{array}$ & $\begin{array}{l}\text { Possible, but only } \\
\text { during imprinting } \\
\text { phase }\end{array}$ \\
\hline $\begin{array}{r}\text { Physiological half- } \\
\text { life }\end{array}$ & $\begin{array}{c}\text { Unmodified - } \\
\text { seconds to minutes } \\
\text { Modified - days }\end{array}$ & Days to weeks & N/A \\
\hline $\begin{array}{l}\text { Application } \\
\text { conditions }\end{array}$ & $\begin{array}{l}\text { Physiological, non- } \\
\text { physiological, with } \\
\text { some application in } \\
\text { organic solvents }\end{array}$ & Physiological & $\begin{array}{c}\text { Mainly organic } \\
\text { solvents, with some } \\
\text { application in } \\
\text { aqueous solutions }\end{array}$ \\
\hline
\end{tabular}

\subsubsection{Systematic Evolution of Ligands by EXponential enrichment (SELEX)}

As above, both DNA and RNA aptamers are selected through $\underline{s y s t e m a t i c} \underline{e}$ volution of ligands by exponential enrichment (SELEX) ${ }^{5-7}$ The general principle of the process is represented in Figure 1.1. Initially, a specific target is exposed to a large random pool consisting of $10^{14}-10^{16}$ oligonucleotides. After incubation, the sequences that exhibit affinity to the target are partitioned from those that either interact weakly or do not bind 
the target. The oligo-target complex is then separated, keeping the sequences that are identified as target binders. These sequences are enriched by polymerase chain reaction (PCR) and the pool, now diminished in diversity, is reintroduced to the target. In the selection of RNA aptamers, extra steps are required and involve initial transcription into RNA library and reverse transcription to allow for PCR amplification. The iterative process is repeated with increasing stringency until strong binders are identified. Several design elements need to be considered in preparation for SELEX. The number of possible sequences within the pool will depend on the length of the central random region, which can range from 20-80 nucleotides long. ${ }^{11,20,21}$ Throughout the randomized region, each position may contain $\mathrm{C}, \mathrm{T}, \mathrm{G}$, or A and the distribution of these is decided based on the desired outcome. The central region is flanked by primer annealing sites which are kept constant due to their required presence in the amplification step..$^{20,21}$ The SELEX methodology is dictated by the characteristics of the target and encompasses factors such as buffer composition and $\mathrm{pH}$, partitioning and recovery methods, as well as conditions including, but not limited to temperature and duration of incubation. ${ }^{16}$ Other important elements include counter and negative selection steps, which ensure that the number of non-specific binders is diminished to negligible amounts. ${ }^{22}$ 


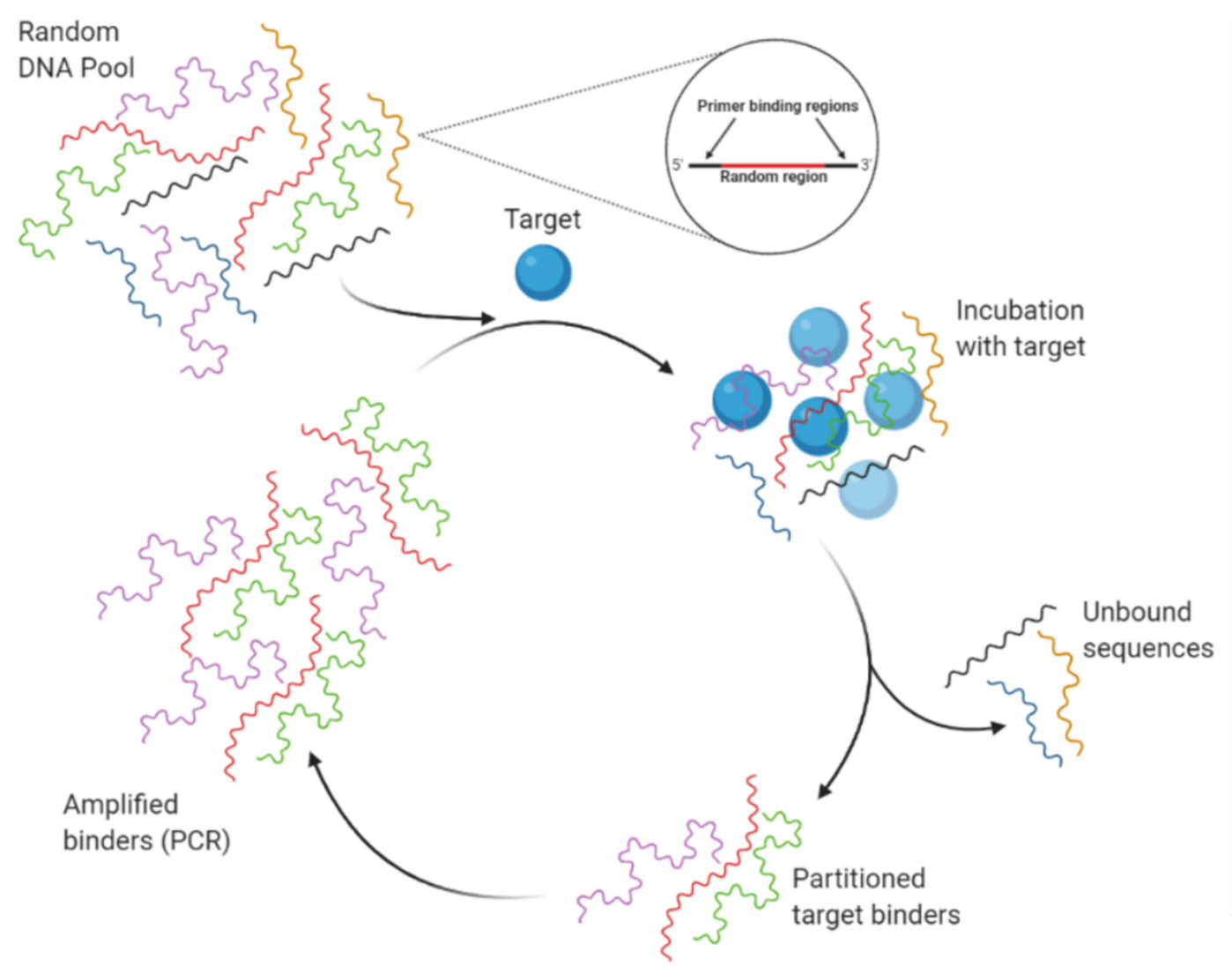

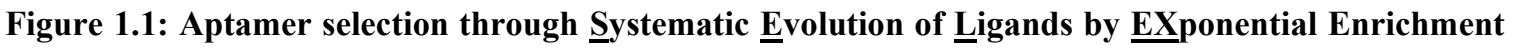
(SELEX). First, a large randomized DNA pool is introduced to the target of interest. Bound sequences are partitioned from those that exhibit weak or no binding. Binders are amplified through PCR and the enriched library is reintroduced to the target. This is repeated for a number of subsequent rounds to select for sequences with the highest binding affinity and selectivity. Figure created in BioRender.

\subsubsection{Considerations for the translation of aptamers to in vivo applications}

Generally speaking, DNA is inherently more stable than RNA, ${ }^{21}$ however, neither of the natural forms are applicable for use in vivo as unmodified nucleic acids are readily degraded by exonucleases and endonucleases in blood and other bodily fluids, and are rapidly cleared based on their small size. ${ }^{23,24}$ To impart sufficient stability and bioavailability, a number of modifications of the bases and/or backbone, as well as terminal caps have been explored. ${ }^{18,23}$ These modifications may either be integrated into the original 
SELEX pool, or incorporated once the selection is complete, ${ }^{18}$ and include 5'- and 3'-end capping, use of mirror image L-DNA, as well as modifications to the nucleobase, phosphodiester backbone, and on the sugar ring. ${ }^{24}$ Nuclease resistance and enhanced cellular availability can be achieved through the incorporation of the internucleotide phosphorothioate linkage. Notably, this type of modification is typically applied to aptamer's niche relative, therapeutic oligonucleotides. The most commonly used aptamer modifications are at the 2'-position of the sugar ring and the simplest modifications are achieved through terminal conjugations. Specifically, with internal modifications, aptamers may be fully or partially substituted with 2'-amino pyrimidines, 2'fluoropyrimidines, and 2'-O-methyl ribose purines and pyrimidines, while 3'-end inverted thymidine and 5'-end polyethylene glycol (PEG) incorporation have shown the most utility as terminal modifications. ${ }^{13}$ The overall goal is to increase nuclease resistance and decrease renal filtration, effectively optimizing tissue residence. ${ }^{13}$ However, in the design of in vivotranslatable oligonucleotides, it is important to always consider the possible undesirable side-effects the endowed modifications may incur. One such example is the recently discovered underlying PEG immunogenicity in humans, which has challenged the widely accepted notion of PEG being non-immunogenic. ${ }^{25}$

\subsubsection{Fibrinogen aptamer SELEX}

Fibrinogen, a $340 \mathrm{kDa}$ glycoprotein, is an important component of circulating plasma as it exists to protect the vascular network from blood loss upon injury. It is a homodimeric protein, which is made up of two sets of each $A \alpha, B \beta$, and $\gamma$ polypeptide chains. The polypeptide chains are assembled into hexameric complexes, $(A \alpha / B \beta / \gamma)_{2}$, with the N-termini located in a central "E nodule" and extended outward in a coiled-coil 
arrangement. ${ }^{26,27}$ This arrangement and its involvement in aptamer binding is further explored in Chapter 4 using Circular Dichroism (CD). Li et al. set out to select a fibrinogenbinding aptamer (FA) under the premise of monitoring the glycosylation site of glycoproteins. ${ }^{28}$ To influence the selection of towards the glycosylation site, modified nucleotides were incorporated into the DNA library. Specifically, boronic-acid modified thymidine-5'-triphosphate (B-TTP) nucleotides were utilized based on their intrinsic ability to interact with diols and single hydroxyl groups. Since glycosylation sites of fibrinogen are located within $\beta$ and $\gamma$ chains, it was postulated that the B-TTP aptamers would gravitate towards those sites. ${ }^{28,29} \mathrm{~A}$ number of candidates were chosen at random and their dissociation constants $\left(\mathrm{K}_{\mathrm{d}}\right)$ were measured. Within the aptamer-target complexes that were tested, the selected candidates showed affinity in the nanomolar range. Fibrinogen aptamer sequence chosen for this work was one that showed good binding affinity towards unmodified fibrinogen target and did not contain the boronic acid modification. The $\mathrm{K}_{\mathrm{d}}$ of this aptamer was measured to be $64 \mathrm{nM}$, and it was the most suitable for proof-of-concept work described below. ${ }^{28}$ The rationale for this choice was based on the proposed application of FA towards not fibrinogen itself but rather for binding the polymerized form, fibrin. We postulated that, in order for the previously selected monomer-binding aptamer to bind the fibrin form, it must be able to bind the structural elements located away from the $\beta$ and $\gamma$ chains, which are modified during the polymerization process (discussed in section 1.4). ${ }^{26,30}$

\subsection{Hemostasis}

The hemostatic system is a tightly regulated process that, under normal circumstances, is only activated in response to tissue damage, generating what could be 
categorized as "good clots". It is comprised of three stages, platelet aggregation, coagulation, and fibrinolysis, otherwise known as primary through tertiary hemostasis. ${ }^{31}$ This is a cascade that is initiated when subendothelial structures are exposed to blood upon physical damage of the vessel wall. Platelets are activated once they adhere to collagen, initiating coagulation signaling, such as $\mathrm{TXA}_{2}$, which leads to increased levels of cytosolic $\mathrm{Ca}^{2+} \cdot{ }^{31}$ Prothrombin undergoes proteolytic conversion to thrombin which is catalyzed by Factor $\mathrm{Xa}$, the result of the extrinsic tenase reaction, assembled into prothrombinase through membrane-dependent interaction with Factor $\mathrm{Va} .{ }^{31,32}$ This in turn originates the activation of fibrinogen, and its subsequent polymerization into fibrin. ${ }^{31}$

Fibrinogen can be classified as an acute phase protein, which means that its plasma concentrations may spike from the typical $2-5 \mathrm{mg} / \mathrm{mL}$ and exceed $7 \mathrm{mg} / \mathrm{mL}$ during inflammation. Fibrinogen circulates in its hexameric complex form until its activated by thrombin, which catalyzes the formation of fibrin protofibrils through half-staggered association of fibrin monomers. This is achieved through the proteolytic cleavage of Nterminus $\mathrm{FpA}$ and $\mathrm{FpB}$ fibrinopeptides from the $\mathrm{A} \alpha$ and $\mathrm{B} \beta$ chains, respectively. Fibrin protofibrils are subsequently aggregated to form the final fibrin meshwork. ${ }^{26}$ Visual representation of fibrin formation is provided in Figure 1.2. Overall, this is a selfamplifying activation system, which would endlessly continue in the absence of inhibition machinery. Finally, fibrinolysis exists to avoid the complete obstruction of the vascular bed with clots, and utilizes plasmin for fibrin degradation after tissue healing. ${ }^{31}$ 


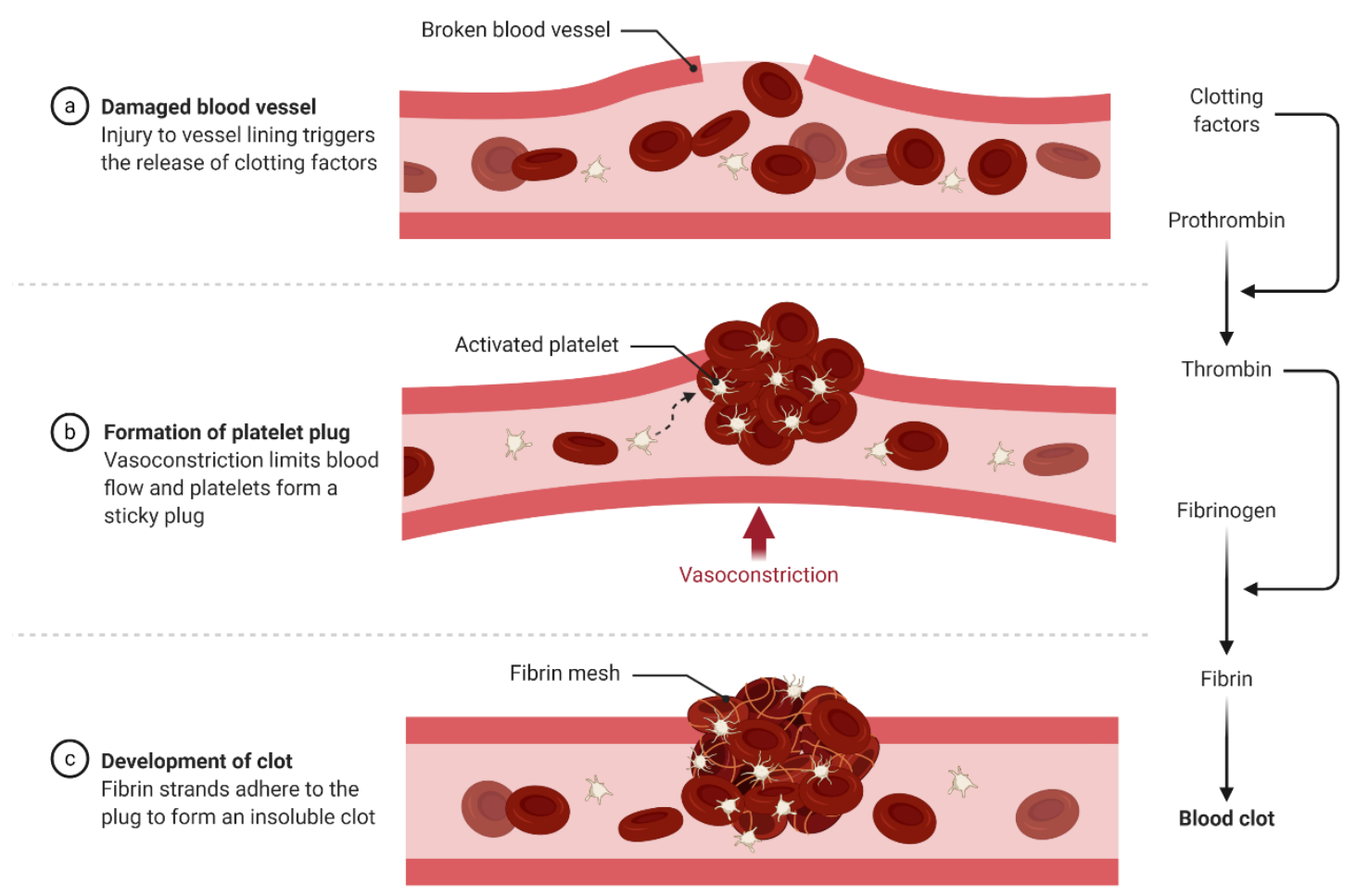

Figure 1.2: Visual representation of secondary hemostasis, where fibrin meshwork is formed through thrombin-catalyzed polymerization of fibrinogen upon blood vessel damage. Imaged prepared in BioRender.

\subsubsection{Hemostatic abnormalities and thrombosis}

The harmony of multiple activators and inhibitors within blood coagulation processes allows for this response to only be activated for the purposes of management of acute events, such as trauma or surgery. However, within the realm of hemostatic malfunctions, two manifestations are possible, with imbalance in procoagulant or anticoagulant components of the system leading to thrombosis, "bad clots", or excessive bleeding. ${ }^{33}$ The biochemical nature of thrombosis must be considered, as it plays an important role in risk evaluation and overall diagnosis. It is well understood that venous thrombi, or "red" thrombi, are mostly composed of fibrin, which also serves to anchor the 
clot to the vessel wall, and entrapped red blood cells. ${ }^{34}$ It is worth noting that fibrin-rich thrombi have been identified as markers of early thrombi that are more susceptible to thrombolytic therapy because of the substantial cross-linked fibrin content. ${ }^{26}$ The major difference between venous and arterial thrombosis is the level of involvement from platelet adhesion. That is, platelets demonstrate little to no contribution towards the formation of venous thrombi, while the primary driving mechanism in the formation of arterial thrombi is platelet deposition. Arterial thrombosis is ordinarily triggered when atherosclerotic plaques rupture, externalizing several components involved in the clotting cascade. Since platelets are largely involved, these clots are known as "white" thrombi. ${ }^{34}$

Hypercoagulability, or propensity to spontaneously develop thrombi, can be caused by hereditary or acquired conditions. Primary hypercoagulable states are related to molecular hemostatic abnormalities, while secondary hypercoagulable states are based on clinical conditions that consequently trigger increased thrombosis. ${ }^{35}$ Within the bounds of primary hypercoagulable states, several players have been identified and in general revolve around defects in the proteins of the coagulation or fibrinolytic systems, but not platelet abnormalities. Overall, deficiencies of the regulatory proteins result in self-propagating production of blood clots in a thrombotic event. Secondary hypercoagulable states encompass a large range of clinical conditions. Most commonly, the pathophysiologic bases of these events are not well defined. These risk factors are summarized in Table 1.2. ${ }^{35,36}$ Random formation of thrombi is classified as a pathological process and results in partial or complete obstruction of blood flow, and limited perfusion. Further complications may include pulmonary embolism, thrombosis-induced myocardial infraction, ischemic stroke, and others. Blood clots also play a major role in hemorrhagic strokes; however, 
their formation can be considered favorable as they serve to seal the site of the hemorrhage, thereby simply accompanying this disease. ${ }^{34,37}$ In all cases, with hemorrhage control and both hypercoagulable states, location and full delineation of the blood clot are considered to be two clinically relevant aspects that could streamline proper diagnosis and treatment follow-up. ${ }^{35,36}$

Table 1.2: Summary of acquired and hereditary risk factors for primary and secondary hypercoagulable states. ${ }^{35,36}$

\begin{tabular}{|c|c|}
\hline $\begin{array}{c}\text { Factors of primary hypercoagulable } \\
\text { states }\end{array}$ & $\begin{array}{c}\text { Factors of secondary hypercoagulable } \\
\text { states }\end{array}$ \\
\hline $\begin{array}{l}\text { - } \begin{array}{l}\text { Disorders of the fibrinolytic system: } \\
0 \text { Hyperhomocysteinemia* } \\
\circ \text { Abnormal plasminogen } \\
\circ \quad \text { Plasminogen activator } \\
\text { deficiency }\end{array} \\
\text { - } \quad \text { Activated protein C resistance/factor } \\
\text { V Leiden } \\
\text { - } \quad \text { Prothrombin gene G20210A mutation } \\
\text { - } \quad \text { Factor XII deficiency } \\
\text { - } \quad \text { Protein C deficiency* } \\
\text { - } \quad \text { Protein S deficiency* } \\
\text { - } \quad \text { Antithrombin III deficiency* } \\
\text { - } \quad \text { Elevated factor VIII activity* } \\
\text { - } \quad \text { Dysfibrinogenemia* }\end{array}$ & $\begin{array}{ll}\text { - } & \text { Major surgery/trauma } \\
\text { - } & \text { Immobilization (e.g., hip/knee } \\
& \text { replacement, prolonged cast, stroke, } \\
\text { bedridden because of illness) } \\
\text { - } \quad \text { Solid or hematologic malignancies } \\
\text { - } \quad \text { Pregnancy } \\
\text { - } \quad \text { Oral contraceptives } \\
\text { - } \quad \text { Estrogen replacement therapy } \\
\text { - } \quad \text { Antiphospholipid antibody syndrome } \\
\text { - } \quad \text { Heparin-induced thrombocytopenia } \\
\text { - } \quad \text { Paroxysmal nocturnal hemoglobinuria } \\
\text { - } \quad \text { Obesity } \\
\text { - } \quad \text { Nephrotic syndrome } \\
\text { - } \quad \text { Smoking }\end{array}$ \\
\hline
\end{tabular}

* Can be hereditary or acquired

\subsubsection{Blood clots in hemorrhagic and ischemic strokes}

Largely, strokes can be categorized into two major subtypes: hemorrhagic and ischemic. Ischemic strokes account for the majority of these events at around $80 \%$, while hemorrhagic strokes serve as the minority at approximately $20 \%$. These proportions may vary based on factors such as socioeconomic status and geographical location. ${ }^{38}$ 
Intracranial hemorrhages may stem from several different causative events, including, but not limited to, trauma, hypertension, or aneurysm. ${ }^{39-41}$ Hypertension, or high blood pressure, is considered to be the most common systemic risk factor leading to intracranial hemorrhages. ${ }^{40,41}$ Intracranial aneurysms are also a widespread risk factor with approximately $10-30 \%$ of those that are affected suffering from multiple aneurysms. ${ }^{42-44}$ Intracranial aneurysm is a cerebrovascular disorder characterized by dilation of cerebral arteries or veins. Although most of these aneurysms are asymptomatic, they tend to grow in an unpredictable manner and even the smallest of dilated vessels can rupture. ${ }^{44-46}$ In the event of a rupture, blood may pool either into the brain parenchyma, resulting in a parenchymal hemorrhage, or the subarachnoid space surrounding the brain between the arachnoid membrane and pia mater, resulting in a subarachnoid hemorrhage (SAH) ${ }^{44}$ Some of the post-rupture complications include re-bleeding, acute hydrocephalus and ischemic injury, as well as non-neurological complications during the acute phase, vasospasm in the subacute phase, and hydrocephalus and cognitive disorders in the chronic phase. ${ }^{47}$

Cardiovascular diseases such as strokes and embolisms have a common underlying pathology of thrombosis. ${ }^{48}$ When considering ischemic stroke, the pathophysiology is typically quite straight forward. There are several physiologic subtypes of thrombosis related to this type of a stroke which, whether they grow locally within the vessel or are the result of an embolism, lead to large vascular occlusions obstructing blood flow. As a result, the portion of the brain that receives diminished supply of blood is deprived of oxygen. ${ }^{49,50}$ Due to low respiratory reserve and complete dependence on aerobic metabolism, the affected region of the brain parenchyma undergoes cellular injury and 
ultimately tissue death and loss of function. ${ }^{50}$ Early thrombolytic intervention or thrombectomy have shown to be effective treatment methods in re-canalization of the occluded vessels. ${ }^{50,51}$ Post-treatment imaging is utilized to follow progression and effectiveness of the treatment. ${ }^{51}$

Overall, the public health burden of strokes is associated with high mortality and morbidity. Approximately $40 \%$ of strokes are fatal, while approximately $60 \%$ of surviving patients are left permanently disabled. The severity of morbidity is determined by how quickly the stroke is diagnosed. ${ }^{38,42,44,45}$ These staggering statistics, along with the existing limitations of the currently existing imaging modalities and toxicities associated with their respective contrast media, which are outlined below, highlight the need for development of techniques that will allow for streamlined diagnosis of the forenamed conditions.

\subsubsection{Diagnosis of hemostatic abnormalities}

Molecular hemostatic abnormalities may be diagnosed through a combination of familial and personal history assessment, and laboratory testing. These protocols are fairly well established. ${ }^{36}$ The work in this thesis focused on imaging of existing blood clots that may be the underlying cause of ischemic stroke or embolism, or those forming at the site of a blood vessel rupture in hemorrhagic stroke. Currently, a number of imaging modalities are used to assess thrombi and the technique of choice is often dictated by the location of the clot. For instance, ultrasound is most commonly used to assess deep vein thrombosis, while strokes are typically diagnosed with computed tomography $(\mathrm{CT}) .^{48}$ Unenhanced CT, while relatively insensitive, shows utility in acute hemorrhage exclusion and is able to pinpoint the general location of hyperdense vessels which indicates clot burden. Contrast enhancement is employed when more advanced diagnosis is required and is obtained 
through the means of angiography. ${ }^{52}$ Specifically, in the early stages of investigation of suspected bleeding within the brain, imaging techniques such as magnetic resonance angiography (MRA), CT angiography, or catheter angiography may be employed. Contrast agents are used to help visualize blood vessels and are injected through a catheter into a vein. This affords the dye direct access to the bloodstream thereby allowing it to quickly reach the brain. ${ }^{44}$ Imaging techniques may be combined with a lumbar puncture in cases of patients presenting with symptoms indicative of a rupture but showing negative results. Although these techniques are effective methods of diagnosis, there are several limitations associated with them. In a lumbar puncture, the origin of the blood within the spinal column is not always specific to a hemorrhage and the test cannot be performed earlier than 6 hours post symptom onset. ${ }^{44}$ Angiography techniques do not determine the exact site of the rupture, especially in patients with multiple aneurisms and can be associated with sideeffects such as acute kidney failure. ${ }^{53}$ The unifying theme of the conditions described above is that their early and accurate diagnosis is critical for treatment and minimizing mortality and morbidity. ${ }^{42,44,45}$

\subsection{Imaging modalities}

A number of different imaging modalities exist and are currently used to distinguish between physiological and pathological tissues and processes. Since their development, magnetic resonance imaging (MRI) and CT imaging have revolutionized the field of medical imaging and are among the most well-established technologies in the field. ${ }^{54,55}$ To allow for better delineation of anatomical structures, and differentiation between normal and pathological tissues, contrast materials are utilized in both. Gadolinium (Gd(III)) can be characterized as the most widespread MRI contrast entity and is administered as part of 
stable chelates with multi-dentate ligands, such as diethylenetriaminepentaacetic acid (DTPA) (Magnevist), 1,4,7,10-tetraazacyclododecane-1,4,7,10-tetraacetic acid (DOTA) (Dotarem) and others (see section 2.6.4.1 for more details). ${ }^{55}$ Iodinated small molecules are among the most commonly used CT contrast agents. In particular, iohexol, sold under the trade name of Omnipaque, is an example of a clinical contrast material that is able to produce contrast enhancement of 25-30 HU per milligram of iodine per milliliter at X-ray energy range of $100-120 \mathrm{kVp}$ (see section 2.7 .1 for more details). ${ }^{55,56}$ Principles behind these imaging modalities and their corresponding contrast materials, including the currently understood strengths and weaknesses, will be discussed in depth in subsequent chapters.

In general, noteworthy limitations of the conventionally used contrast agents include lack of specificity towards the pathological tissue, rapid clearance which necessitates high administration doses, and toxic effects, especially as it pertains to tissue accumulation. ${ }^{55,57,58}$ With that in mind, there has been a major push towards the development of targeted contrast agents in order to overcome these pitfalls. ${ }^{55}$ Targeting ligands, such as antibodies, proteins, affibodies, aptamers, peptides, small molecules and others have been researched (Figure 1.3). 

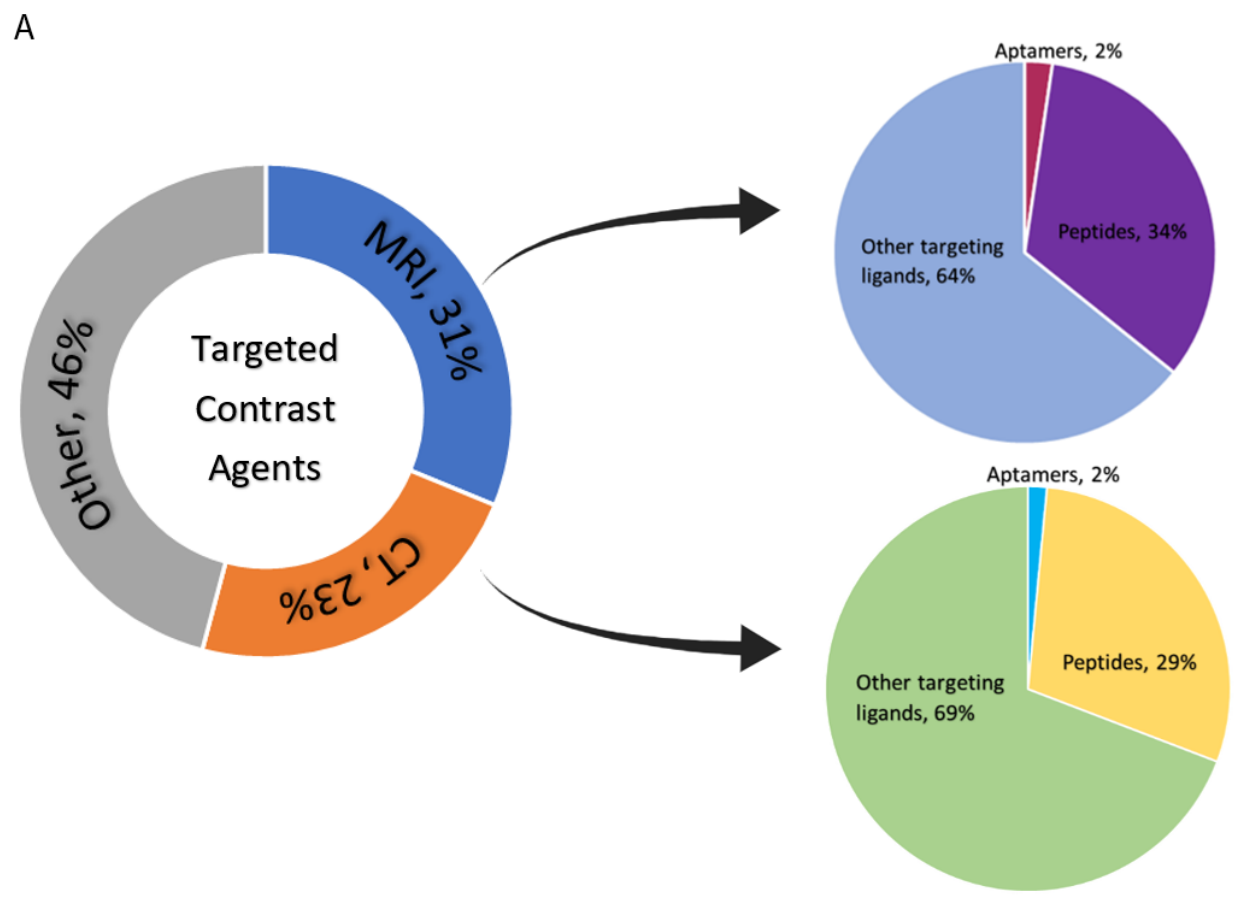

B
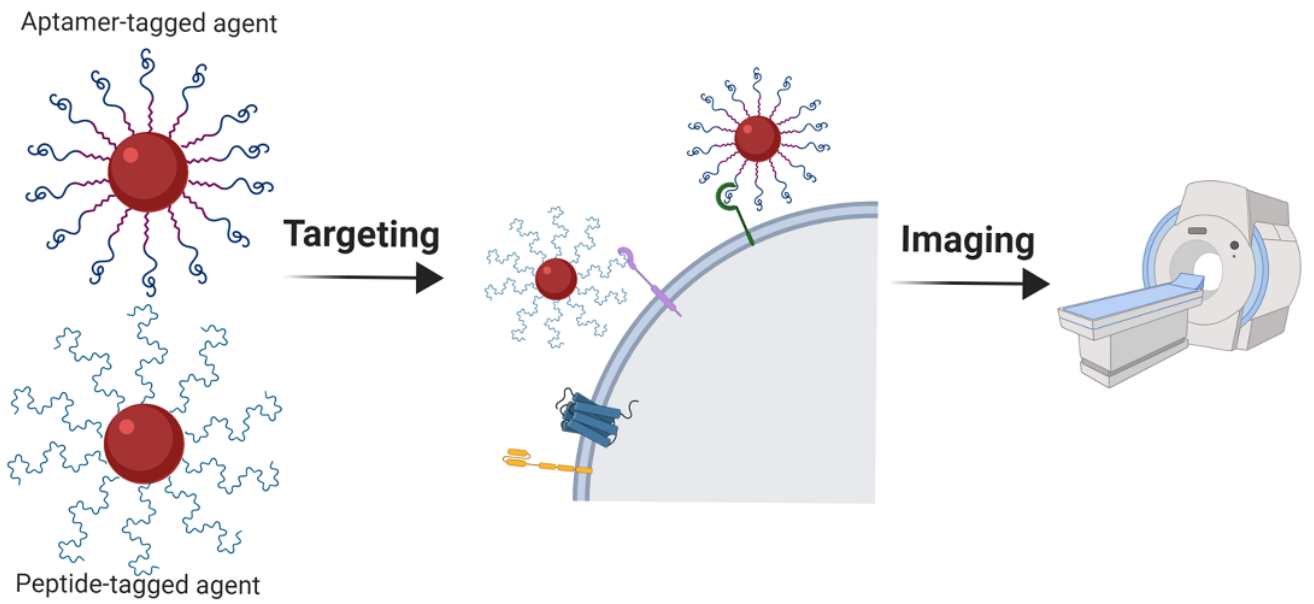

Figure 1.3: (A) Targeted contrast agents developed towards MRI and CT applications make up more than $50 \%$ of the research in the field over the last 3 years. About a third of these utilize aptamers or peptides as their targeting moiety. Figure developed based on SciFinder search output. (B) Graphic illustration of aptamer- and peptide- targeted contrast agents for MRI and CT imaging. Image made in BioRender. 
Generally, antibodies or proteins tend to be the targeting agent of choice. For example, antibodies have been found to be effective in oncological imaging, and a comprehensive review was written by Jason M. Warram, while protein-gadolinium formulations have been utilized for MRI, which was outlined by Sashiprabha Vithanarachchi. ${ }^{59,60}$ Affibodies are another example of an affinity ligand which allows for cancer-associated molecular imaging. They are small (58 amino acids) protein scaffolds engineered to bind a variety of protein targets. In fact, ${ }^{68} \mathrm{Ga}$-labelled affibody molecules have gone as far as Phase I/II clinical testing and demonstrated accurate and specific measurement of HER2 expression in positron emission tomography (PET) imaging. A review by Vladimir Tolmachev and Anna Orlova discusses principles and properties of these molecules. ${ }^{61}$ Other affibody formulations have been explored, including those useful in MRI, CT, and single-photon emission computed tomography (SPECT). ${ }^{62-64}$ Nevertheless, peptides and aptamers strike a balance with their properties in comparison to their counterparts. Comparatively, aptamers and peptides demonstrate reasonable clearance times, based on their smaller size, while exhibiting tight target binding to a range of targets, which is not often achieved by small molecules. ${ }^{54}$ The subsequent sections will give a basic overview of nano-based approaches for MRI and CT imaging and outline some recent advancements in peptide- and aptamer-targeted imaging agent formulations aimed towards streamlining accurate diagnosis of disease states.

\subsubsection{Nano-contrast agents}

In recent years, the development of nanomedicine has taken center stage, demonstrating utility in many areas, including diagnostic imaging. Unsurprisingly, nanoformulations are at the forefront of research towards the development of novel contrast 
materials for medical imaging as they allow for improvement of sensitivity, biocompatibility, and biodistribution. ${ }^{65}$ A major advantage of these formulations is that nanomaterials exhibit a phenomenon termed as the enhanced permeability and retention (EPR) effect. Namely, due to tumor leaky vasculature, nano-formulations can preferentially accumulate in tumor vessels through active or passive targeting while their retention is accomplished based on the lack of functional lymphatic drainage. ${ }^{66,67}$ In addition, nano-formulations allow for surface-functionalization which streamlines diagnostic capabilities of various disease states. ${ }^{65}$

One such nanosubstance that has been proposed as an effective MRI contrast agent is superparamagnetic iron oxide nanoparticles (SPIONs). Generally, there are three major factors that dictate efficacy of SPIONs, including particle size, composition, and crystallinity. An important consideration in their design is that SPIONs start to lose their superparamagnetic properties beyond $20 \mathrm{~nm}$ in diameter. ${ }^{68}$ This size dependence is defined by a threshold, below which magnetic nanoparticles remain single-domain, which ensures that the coherent rotation of spins occurs as a unit in all magnetic fields. ${ }^{69,70}$ Overall, iron oxide is extremely susceptible to an external magnetic field based on the presence of 4 or 5 unpaired electrons in the $3 \mathrm{D}$ shell for $\mathrm{Fe}^{2+}$ or $\mathrm{Fe}^{3+}$ states, respectively, with $\mathrm{Fe}_{3} \mathrm{O}_{4} \mathrm{NPs}$ yielding stronger T2 shortening effect than that of $\gamma-\mathrm{Fe}_{2} \mathrm{O}_{3}$ (T2 relaxation is explained in sections 2.6.2 and 2.6.4.2). ${ }^{68}$ Unfortunately, with negative contrast agents producing a dark signal, it can at times be difficult to distinguish them from an artifact or an air bubble. Despite these limitations, SPIONs have proven themselves as an effective alternative to other contrast agents as they offer high sensitivity and excellent biocompatibility. Commercially available examples include Feridex and Revolist. ${ }^{68,71}$ 
Another promising type of MRI nano-contrast material is gadolinium oxide nanoparticles $\left(\mathrm{Gd}_{2} \mathrm{O}_{3} \mathrm{NPs}\right)$, displaying superior $\mathrm{T} 1$ contrast enhancement to that of $\mathrm{Gd}^{3+}-$ chelates ( $\mathrm{T} 1$ relaxation is explained in sections 2.6.2 and 2.6.4.1). The function of these nanoparticles is governed by the same principle as the latter, but the enhanced T1 contrast is based on the high density of $\mathrm{Gd}^{3+}$ present within each nanoparticle. A simple example was investigated by Dai and colleagues, assessing the toxicity, pharmacokinetics, biodistribution, and other properties of poly-(ethylene glycol) (PEG)-modified $\mathrm{Gd}_{2} \mathrm{O}_{3} \mathrm{NPs}$. PEG is common surface coating used to stabilize nanoparticles, enhance their biocompatibility, and prolong their blood circulation time. This research confirmed that PEGylated $\mathrm{Gd}_{2} \mathrm{O}_{3}$ NPs display longer half-life than $\mathrm{Gd}^{3+}$-chelates, thereby offering greater accumulation at tumor sites, low histological, hepatic and renal toxicity, and efficient MR contrast enhancement. ${ }^{72}$

Other formulations of MRI nano-contrast agents have been proposed, including ones based on highly paramagnetic ions such as manganese as a possible T1 agent, and dysprosium as a possible T2 agent. In all cases, since these formulations are nanoparticlebased, the final contrast formulation can be surface-modified to produce targeted contrast, dual-contrast, or drug-carrying contrast. ${ }^{73}$ Liposomal formulations are an excellent example of substance-carrying nanoparticles, with a surface that can be functionalized, as well as a hollow interior that can be loaded with various molecules. As a result, liposomes can easily act as dual-mode imaging probes and image-guided drug delivery agents. A review, written by Xia et al. outlines the design of these probes, geared towards clinical applications. ${ }^{74}$ 
When considering conventional contrast media in $\mathrm{CT}$, one of the limitations is lack of selectivity between healthy and pathological tissues. Gold nanoparticles (AuNPs) are an attractive alternative due to their reactive surface which allows for incorporation of a large variety of targeting molecules. Other advantages include inherently high X-ray adsorption coefficient, electron density and atomic number; a combination of these characteristics results in X-ray attenuation which tends to be stronger than that of iodine-based molecules. ${ }^{75,76}$ Wang and colleagues designed one such contrast agent consisting of hollow gold nanoparticles (HGNPs) surface-modified with PEG, mPEG@HGNPs. The final mPEG@HGNPs were $63.4 \mathrm{~nm}$ in diameter and demonstrated concentration-dependent increase of X-ray attenuation which was higher than that of the commercially available control. In vivo imaging further confirmed contrast efficiency while exhibiting good biocompatibility. ${ }^{77}$ A plethora of other GNP-based contrast agents are being researched, bearing various surface-modifications which are often used as another approach to further enhance X-ray attenuation. ${ }^{76}$

\subsubsection{Targeted contrast for molecular imaging}

\subsubsection{Peptide targeting examples}

This section will review the recent examples of MRI, CT, and multi-modal formulations which were designed for molecular imaging applications, targeting known pathological markers with peptides that were developed to bind them. Table 1.3 highlights the important features of these formulations. Not all of these features are discussed in each paper; however, this summary can be used as a guideline for the design of future studies, with these important elements in mind. 
Table 1.3: Summary of recently developed peptide-targeted contrast agents for applications in MRI, CT, and dual-imaging of various molecular markers of disease.

\begin{tabular}{|c|c|c|c|c|c|c|c|c|}
\hline $\begin{array}{r}\text { Peptide-targeted } \\
\text { contrast }\end{array}$ & Imaging target & $\begin{array}{c}\text { Peptide } \\
\text { name }\end{array}$ & $\begin{array}{c}\text { Amino } \\
\text { Acid } \\
\text { length }\end{array}$ & Modality & $\begin{array}{l}\text { Contrast } \\
\text { material }\end{array}$ & $\begin{array}{c}\text { Injected } \\
\text { dose }\end{array}$ & $\begin{array}{c}\text { Circulation } \\
\text { half-life }\end{array}$ & Ref \\
\hline $\begin{array}{r}\text { exendin-4- } \\
\operatorname{dota}(g a)-G d(I I I) \\
(G d E x)\end{array}$ & GLP-1R & Exendin-4 & 39 & MRI & Gd(III) & $\begin{array}{l}1.12 \mu \mathrm{mol} \\
\text { /animal }\end{array}$ & N/A & {$\left[{ }^{78}\right]$} \\
\hline $\begin{array}{r}p H L I P- \\
\text { magnetosomes }\end{array}$ & $\begin{array}{c}\text { Tumor } \\
\text { extracellular } \\
\text { environment }\end{array}$ & pHLIP & 38 & MRI & $\begin{array}{l}\text { Magnetosome } \\
\left(\mathrm{Fe}_{3} \mathrm{O}_{4}\right)\end{array}$ & $\begin{array}{c}0.5 \mathrm{mg} \\
\mathrm{Fe} / \mathrm{mL} \text { or } \\
1.5 \mathrm{mg} \\
\mathrm{Fe} / \mathrm{mL}\end{array}$ & $\begin{array}{c}\text { Cleared within } \\
\sim 24 \mathrm{hrs}\end{array}$ & {$\left[{ }^{79}\right]$} \\
\hline $\begin{array}{r}N a G d F_{4} @ b p- \\
\text { peptide NPs }\end{array}$ & p32 & bp-peptide & 9 & MRI & $\mathrm{NaGdF}_{4}$ & $10 \mathrm{mg} \mathrm{Gd} / \mathrm{kg}$ & $\mathrm{N} / \mathrm{A}$ & {$\left[{ }^{80}\right]$} \\
\hline $\begin{array}{c}\mathrm{FITC}-\mathrm{VHP}- \\
\mathrm{Fe}_{3} \mathrm{O}_{4} @ \mathrm{SiO}_{2}\end{array}$ & VCAM-1 & VHP & 7 & MRI & $\mathrm{Fe}_{3} \mathrm{O}_{4} @ \mathrm{SiO}_{2}$ & $\begin{array}{l}2.5 \mathrm{mg} \\
\mathrm{NPs} / \mathrm{kg}\end{array}$ & $\mathrm{N} / \mathrm{A}$ & {$\left[{ }^{81}\right]$} \\
\hline$c R G D-G d N P s$ & $\begin{array}{c}\alpha_{\mathrm{V}} \beta_{3} \text { and } \alpha_{\mathrm{V}} \beta_{5} \\
\text { integrins } \\
\end{array}$ & cRGD & 3 & MRI & $\mathrm{Gd}_{2} \mathrm{O}_{3}$ & $\begin{array}{c}0.1 \mathrm{mmol} \\
\mathrm{Gd} / \mathrm{kg} \\
\end{array}$ & $\begin{array}{c}\text { Cleared within } \\
\sim 24 \mathrm{hrs} \\
\end{array}$ & {$\left[{ }^{82}\right]$} \\
\hline$\overline{T-G N P-A B P S}$ & Cathepsin & $\begin{array}{c}\mathrm{GB} 111- \\
\mathrm{NH}_{2}\end{array}$ & 3 & $\overline{\mathrm{CT}}$ & AuNPs & $\begin{array}{c}25 \mathrm{mg} / \mathrm{mL}(5 \\
\mathrm{mg} \text { of GNP } \\
\text { per mouse })\end{array}$ & $\overline{\mathrm{N} / \mathrm{A}}$ & {$\left[{ }^{83}\right]$} \\
\hline $\begin{array}{r}G N P S_{-} \\
P E G @ c N G R \\
\end{array}$ & $\begin{array}{c}\text { Aminopeptidase- } \\
\mathrm{N}\end{array}$ & SH-cNGR & 3 & CT & AuNPs & $10 \mathrm{mg} / \mathrm{mL}$ & $\mathrm{N} / \mathrm{A}$ & {$\left[{ }^{84}\right]$} \\
\hline $\begin{array}{r}c N G R- \\
A u: G d @ G S H \\
\end{array}$ & CD13 receptor & $\overline{\mathrm{cNGR}}$ & 3 & $\begin{array}{c}\text { Multi-modal } \\
\text { (MRI and CT) }\end{array}$ & $\begin{array}{l}\mathrm{Au} \text { and } \mathrm{Gd} \\
\text { atom cluster }\end{array}$ & $\begin{array}{l}5 \mathrm{mM} \mathrm{Au}, \\
16 \mathrm{mM} \mathrm{Gd}\end{array}$ & $19.33 \mathrm{hrs}$ & {$\left[{ }^{85}\right]$} \\
\hline $\begin{array}{r}p E G F R \text {-targeted } \\
\mathrm{Ba}_{2} \mathrm{GdF} \mathrm{F}_{7} \mathrm{NPS}\end{array}$ & EGFR & pEGFR & 14 & $\begin{array}{l}\text { Multi-modal } \\
\text { (MRI and CT) }\end{array}$ & $\mathrm{Ba}_{2} \mathrm{GdF}_{7}$ & $20 \mathrm{mg} \mathrm{Gd} / \mathrm{kg}$ & $\begin{array}{c}\text { Cleared within } \\
24-48 \mathrm{hrs}\end{array}$ & {$\left[{ }^{86}\right]$} \\
\hline $\begin{array}{r}R G D-P E G_{-} \\
B a G d F_{5}\end{array}$ & $\alpha_{\mathrm{v}} \beta_{3}$ & RGD & 3 & $\begin{array}{l}\text { Multi-modal } \\
\text { (MRI and CT) }\end{array}$ & $\mathrm{BaGdF}_{5}$ & $\begin{array}{c}150 \mathrm{mg} \\
\mathrm{Gd} / \mathrm{kg}\end{array}$ & $1.26 \mathrm{hrs}$ & {$\left[{ }^{87}\right]$} \\
\hline
\end{tabular}


A recent example of a novel peptide-targeted MRI probe was published by Clough et al., highlighting the need to quantitatively assess the changes in mass of $\beta$-cells, which is associated with the development of diabetes. ${ }^{78}$ They proposed employing a peptide, exendin-4, for targeted delivery of the MRI probe. Exendin-4 is composed of 39 amino acids; it is a potent analog of the peptide which binds to glucagon-like-peptide-1 receptor (GLP-1R). Since GLP-1R is highly expressed on $\beta$-cells, it can be used as a molecular marker. Notably, exendin-4 has shown utility in treatment of diabetes in addition to demonstrating anti-apoptotic function. As outlined by the authors, its conjugation to numerous therapeutic and imaging agents has been validated by several groups. ${ }^{78}$ In this work, the synthesis and characterization of the exendin-4-conjugated 4-DOTA(ga)-Gd(III) (GdEx) complex as well as its subsequent behavior in vivo using $\beta$-cell-depleted C57BL/6J mice were reported. GdEx demonstrated a significantly stronger brightening enhancement when compared to the commercially available $[\mathrm{Gd}(\mathrm{DOTA})]^{-}$contrast at the $18-21$ minute timepoint and exhibited a slower decline to the baseline value up to the 54 minute timepoint. This was likely correlated with selective accumulation of GdEx in the pancreas from localization to the $\beta$-cells. To assess whether GdEx can be used to detect the decrease in $\beta$-cells mass, mouse strain with tamoxifen-inducible, $\beta$-cell-specific, Dicer deletion ( $\beta$ Dicer-null) was utilized. $\beta$ Dicer-null mice exhibited depleted $\beta$-cells mass and were strongly hyperglycemic. When injected with GdEx, decreased pancreatic uptake was observed in comparison to the control mice with the most significant trends evident at 1821 minutes (approximately 1.8-fold lower) up to a complete wash-out by 54 minutes. This proof-of-concept study allows for the development of similar probes that would allow monitoring of physiological changes overtime. ${ }^{78}$ 
One of the applications of MRI contrast agents is in cancer diagnosis, allowing to delineate tumour size, shape, and overall location. This information is subsequently used for planning and monitoring of treatment. Schuerle and colleagues proposed targeting of the well-understood extracellular acidity associated with tumors with genetically encoded $\mathrm{pH}$ low insertion peptide (pHLIP). ${ }^{79}$ In order to achieve dramatic contrast enhancement, they used magnetosomes, which are particles harvested from magnetotactic bacteria (MTB). Specifically, MTB produce magnetite $\left(\mathrm{Fe}_{3} \mathrm{O}_{4}\right)$ in a chain surrounded by a phospholipid bilayer membrane, forming the magnetosome. Magnetospirillum magneticum AMB-1 cells were genetically engineered to display pHLIP on the surface of magnetosomes to achieve functionalization. The ability of pHLIP-decorated magnetosomes to bind cells in an acidic environment was tested in vitro using the human breast cancer cell line MDA-MB-231, both at a standard $\mathrm{pH}$ of 7.5 , and at a slightly acidic $\mathrm{pH}$ of 6.5 , which is representative of average tumor acidity. Binding of pHLIPmagnetosomes was shown to be significantly higher at low $\mathrm{pH}$ than at neutral $\mathrm{pH}$, as well as when comparing to native magnetosomes at both $\mathrm{pH}$ levels. Subsequent in vivo efficiency was tested using mice bearing flank xenografts of the human ductal carcinoma cell line MDA-MB-435S. Intravenous injections were followed by biodistribution assessment at 6- and 24-hours post injection. Approximately 1.5-fold increase of tumoral accumulation was achieved in the presence of hHLIP functionalized magnetosomes, when compared to the control. Finally, a 2.02-fold higher T2 contrast enhancement was achieved when pHLIP functionalized magnetosomes preferentially accumulated at the site of the tumor. These results demonstrate that this versatile and flexible platform can be broadly applied towards tumor-selective MRI. ${ }^{79}$ 
Chen et al. proposed using $\mathrm{NaGdF}_{4}$ nanoparticle-based formulation to produce a tumor-specific multifunctional nanotheranostic agent. ${ }^{80}$ The composite contrast agent consisted of the $9 \mathrm{~nm} \mathrm{NaGdF}_{4}$-core for T1-weighted MR imaging, decorated with linearlylinked p32 protein binding and pro-apoptotic peptides. More specifically, tumor-homing motif was designed to target mitochondrial p32, a critical regulator of tumor metabolism, while the pro-apoptotic segment produced the therapeutic effect. Cytotoxicity and internalization capabilities of the final complex, denoted $\mathrm{NaGdF}_{4} @$ bp-peptide NPs, was assessed using human breast cancer cells (MCF-7). Results showed that $\mathrm{NaGdF}_{4} @$ bppeptide NPs exerted significant toxic effects on tumor cells, while remaining relatively non-toxic to normal cells. This was related to high targeting efficiency of $\mathrm{NaGdF}_{4} @$ bppeptide NPs, which was associated with the presence of the surface recognition elements. Although $\mathrm{NaGdF}_{4} @$ bp-peptide NPs exhibited relatively low relaxivity values, likely due to the highly branched structure of the peptides, in vitro MRI showed significantly higher signal intensities and cellular internalization in treatments with $\mathrm{NaGdF}_{4} @$ bp-peptide NPs when compared to controls. In vivo studies were conducted using BALB/c mice that were subcutaneously inoculated with MCF-7 cell. Strongly positive MR contrast enhancement was observed at the tumor location in T1-weighted images, confirming tumor-targeting capacity of $\mathrm{NaGdF}_{4} @$ bp-peptide NPs. Finally, ex vivo assessment of the tumor volume confirmed that treatment with $\mathrm{NaGdF}_{4} @$ bp-peptide NPs results in tumor growth suppression without systemic toxicity. This study reveals that combining targeting and therapeutic elements allows for production of nanotheranostic complexes without compromising the efficacy of either elements. ${ }^{80}$ 
Another example of peptide-targeted contrast agent was formulated to be applied towards atherosclerosis plaque imaging. ${ }^{81}$ The vascular cell adhesion molecule 1 (VCAM$1, \mathrm{CD} 106)$ is a prominent marker of atherosclerotic plaque development and can therefore be used for targeted diagnosis. In fact, its high expression persists in both early and late stages of atherosclerosis. Peptide-modified magnetic mesoporous silica nanoparticles were developed by $\mathrm{Xu}$ et al. Specifically, $\mathrm{Fe}_{3} \mathrm{O}_{4} @ \mathrm{SiO}_{2} \mathrm{NPs}$ were surface-functionalized with VHPKQHR peptide (FITC-VHP-Fe $\mathrm{O}_{4} @ \mathrm{SiO}_{2}$ ), which possesses targeting capabilities towards VCAM-1 that are very analogous to those of an antigen-4. In vitro analysis was conducted using mouse aortic endothelial cells (MAECs) treated with lipopolysaccharide to induce high expression of VCAM-1. The results confirmed that VHP-modified nanoparticles are able to efficiently target atherosclerosis while experiencing low toxicity and good blood compatibility. In vivo imaging was used to confirm targeting and retention of FITC-VHP-Fe $\mathrm{O}_{4} @ \mathrm{SiO}_{2}$, following a tail vein injection into $\mathrm{ApoE}^{-/-}$mice that were fed a high-fat diet. This confirmed that FITC-VHP-Fe $\mathrm{O}_{4} @ \mathrm{SiO}_{2}$ was highly specific towards VCAM-1, while a significant reduction in T2 relaxation time (negative enhancement by approximately $33 \%$ ) revealed that this type of formulation is superior to commercially available contrast media when used for the diagnosis of atherosclerotic plaques. Finally, targeting capabilities were further reinforced ex vivo using the fluorescence component of FITC-VHP-Fe $\mathrm{O}_{4} @ \mathrm{SiO}_{2}$ when the aortic vessels were harvested and imaged using $470 \mathrm{~nm}$ excitation and $535 \mathrm{~nm}$ emission wavelengths. This supports that choosing a molecular marker that is specifically expressed during a particular stage of disease progression could allow for precise monitoring and more effective diagnosis. ${ }^{81}$ 
In a different example of peptide-guided contrast enhancement, ultra-small gadolinium oxide $\left(\mathrm{Gd}_{2} \mathrm{O}_{3}\right)$ nanoparticles (GdNPs) were applied towards targeted tumor imaging. Gadolinium affords these nanoparticles a wide range of properties, including the ability to produce positive MRI contrast (T1) based on the spin magnetic moment. Ahmad and colleagues proposed coating GdNPs with cyclic RGDs (cRGDs), cyclic arginineglycine-aspartic acid peptides, for the purposes of targeting tumors through binding to $\alpha_{\mathrm{V}} \beta_{3}$ and $\alpha_{v} \beta_{5}$ integrins overexpressed in tumor angiogenic sites and tumor cells. Both longitudinal and transverse relaxivities of these 1-2.5 nm diameter cRGD-GdNPs were evaluated, demonstrating 3-5 times higher water-proton relaxivities than $\mathrm{Gd}^{3+}$-DTPA and $\mathrm{Gd}^{3+}$-DOTA controls. In vitro imaging of U87MG tumor cells confirmed significant internalization of cRGD-GdNPs, which suggests that this type of complex can be used for gadolinium neutron capture therapy. In vivo evaluations were performed using the orthotopic mouse liver tumor model, where nude mice inoculated with HepG2-luc2 cells. Clear positive contrast enhancement was observed as soon as 5 minutes post injection of cRGD-GdNPs, with 3-times higher accumulation at the tumor sites when compared to normal regions of the liver. These results confirmed that Gd-based nanoparticles can be used as MRI contrast agents, and their surface functionalization results in effective targeting. ${ }^{82}$

Visualization and characterization of distinct molecular pathways in various disease models allows for assessment of those diseases, which ultimately streamlines early diagnosis and improves prognosis. For example, it has been shown that the activity of cathepsin protease is commonly elevated within tumor microenvironments. Utilizing this unique change within the cancerous tissue, cathepsin targeting can be used for detection 
and assessment of tumors. This was the goal of Tsvirkun and colleagues, who proposed using surface-modified GNPs as the contrast agent for non-invasive cancer CT imaging. ${ }^{83}$ GNPs were modified with a cathepsin inhibitor, GB111- $\mathrm{NH}_{2}$. GB111- $\mathrm{NH}_{2}$ is a covalent targeting moiety and is an active site inhibitor specific to cathepsin, thereby acting as an activity-based probe (ABP). The recognition element for cathepsins was the short peptide portion of the probe (carbobenzoxy-phenylalanine-lysine) while the acyloxymethyl ketone served as a warhead, enabling covalent linkage of the probe to its target. Targeting of GNPs to the tumor site was designed to result in specific accumulation of GNPs thus increasing contrast and sensitivity of each CT scan. In vitro and in vivo testing was performed, using NIH-3T3 and 4T1 cells, and BALB/C mice subcutaneously expressing 4T1 cells, respectively. T-GNP-ABPs showed minimal cytotoxicity, as well as size-dependent accumulation in subcutaneous tumors with highest accumulation of up to $12 \%$ of the tumor volume for the smaller particles. Interestingly, it was discovered that CT contrast of the tumor was inversely proportional to GNP size and amount of targeting moiety. That is, the 10-30 nm T-GNP-ABPs were the most effective for tumor imaging, based on their specific tumor uptake. Since this study explored different variants of the contrast formulation, testing different sizes of GNPs and variable proportions of the protective PEG coating and GB111- $\mathrm{NH}_{2}$, it can serve as a guide for future designs of activity-based probes. ${ }^{83}$

One of the critical processes in cancer progression is tumor angiogenesis, during which the tumor grows, develops, and eventually metastasizes. Currently, quantitative studies of angiogenesis are based on the measurement of microvascular density, which is calculated using the maximal number of vasculatures per unit area of histological tissue section. This approach is considered to be invasive and unreliable, as it requires an intact 
tissue sample, and it is not able to account for tissue heterogeneity. Wu and colleagues proposed using $\mathrm{CT}$ as a non-invasive method of visualization of tumor vascularization, developing contrast media that allowed for imaging with high sensitivity and specificity, and simultaneous high retention. ${ }^{84}$ Their formulation was based on surfacefunctionalization of gold nanoparticles with cyclized asparagine-glycine-arginine (SHcNGR) which was used as the tumor-seeking moiety, and carboxypoly(ethylene glycol)thiol (SH-PEG-COOH) in order to increase circulation time of the contrast media. The final contrast probe, GNPs-PEG@cNGR, was used for targeting aminopeptidase-N (APN/CD13), a peptide overexpressed in the endothelium of tumor angiogenesis. In vitro cytotoxicity and targeting specificity assessments were done using CD13-positive cell lines, HepG2, HUVEC. Results showed that GNPs-PEG@cNGR had no observable toxic effect on neither tumor nor control tissue which represented microvasculature and renal epithelial cells. In addition, GNPs-PEG@cNGR was able to effectively target CD13, resulting in efficient intracellular accumulation. The effectiveness of the probe as a CT contrast agent was tested in vivo using a subcutaneous xenograft model, targeting 4T1 tumors in mice. The GNPs-PEG@cNGR was able to bind the tumor vasculature with high specificity, demonstrating rapid and elevated tumor uptake with a $64.9 \%$ increase of contrast enhancement of neovascularity after 1 hour and $96.5 \%$ increase after 4 hours. ${ }^{84}$

A similar method of assessment of tumor angiogenic activity based on endothelial cell targeting was developed by Li et al. ${ }^{85}$ It was proposed that a multimodal contrast agent, utilizing MRI and CT imaging techniques, can be formulated in order to augment tumor vasculature and provide complementary information for a more detailed final assessment. The contrast agent consisted of a gold and gadolinium atom cluster (Au:Gd), 
coated by a naturally occurring zwitterionic glutathione (GSH) and surface-modified with cyclic NGR peptide (cNGR) (Figure 1.4 A). Au was incorporated to serve as the CT contrast while Gd acted as the MRI contrast, with both ions protected by the outer shell. As in previous formulations, cNGR was used for targeting the CD13 receptor, overexpressed on the tumor surface, to achieve improved cellular binding and tumor retention. Improved longitudinal relaxivity was observed when compared to Magnevist in phantom MRI scans (Figure 1.4 C), and higher X-ray attenuation was evident when compared to iohexol in phantom CT scans (Figure 1.4 B). More specifically, in phantom MRI, T1 proton relaxation of cNGR-Au:Gd@GSH was approximately 8.6 times higher than that of Magnevist when scanned at the same concentrations, while in phantom CT, improvement of X-ray attenuation varied between 18 and 180 times of that of iohexol, depending on the $\mathrm{CT}$ sequence used. In vitro assessments revealed that cNGRAu:Gd@GSH experiences highly specific binding towards HepG2, while remaining nontoxic. In vivo MR and CT imaging, using mice with orthotopically implanted EMT-6 cells, clearly demonstrated accumulation of cNGR-Au:Gd@GSH at the tumor sites 8 hours post injection (Figure 1.4 D, E). With both imaging modalities, observable contrast and relative signal intensities were significantly higher than the untargeted control treatments. Overall, this formulation displayed high clinical potential, allowing for specific targeting, improved contrast enhancement in both MRI and CT while highlighting tumor angiogenic vessels for at least a 24-hour period. ${ }^{85}$ 

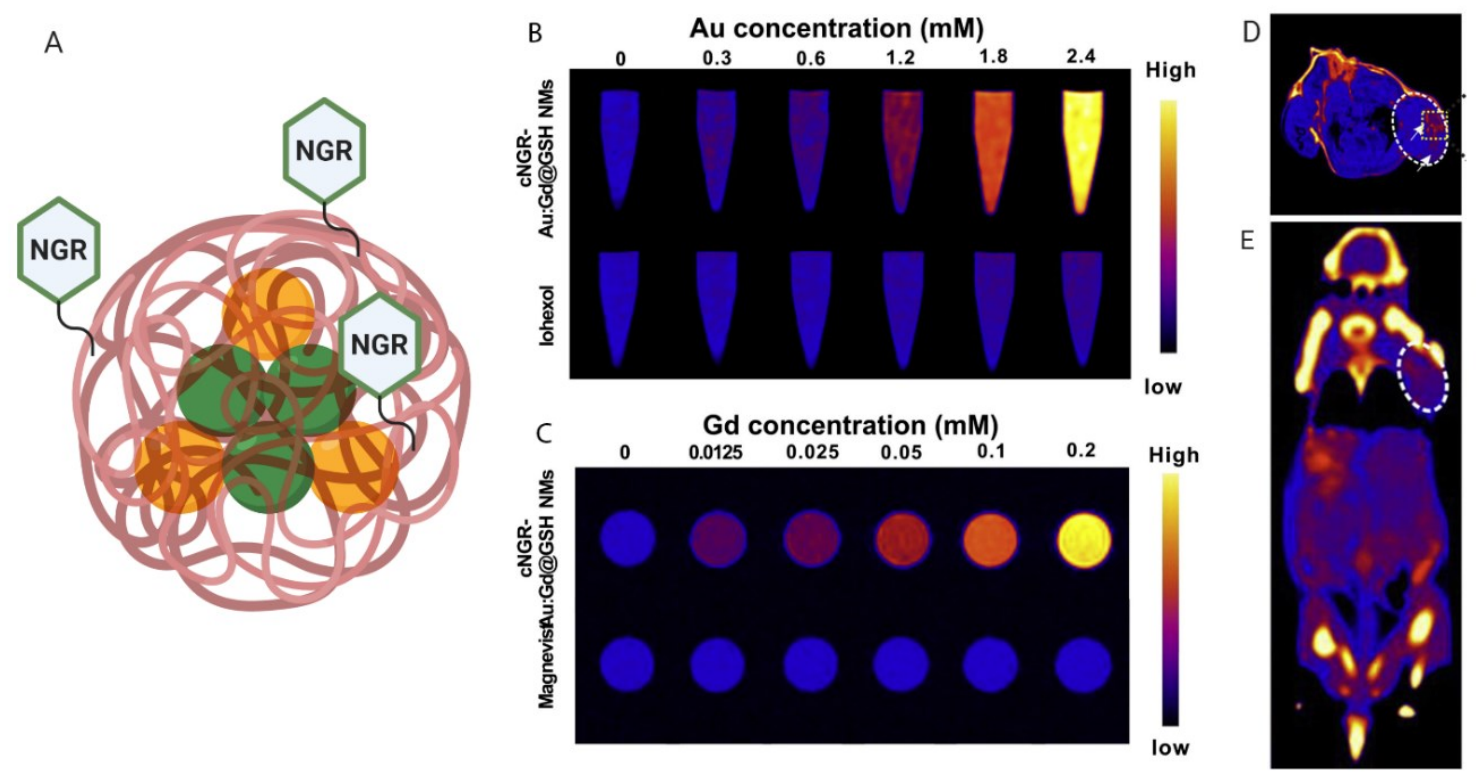

Figure 1.4: (A) Illustration of the Au:Gd@GSH nanoclusters, labelled with cyclic NGR peptide, used for dual mode imaging. (B) CT contrast phantom images of cNGR-Au:Gd@GSH NMs compared to iohexol. (C) MR contrast phantom images of cNGR-Au:Gd@GSH NMs compared to Magnevist. (D) MR image of tumor-bearing mouse at $24 \mathrm{~h}$ post injection of Au:Gd@GSH-NGR, with contrast highlighting the location of the tumor in the right shoulder. (E) CT image of tumor-bearing mouse 24 h post injection of Au:Gd@GSH-NGR, with contrast highlighting the location of the tumor in the right shoulder. Adapted with permission from Elsevier. ${ }^{85}$

Another way to combine the strengths of MRI and CT imaging is through the use of nanoparticles such as $\mathrm{Ba}_{2} \mathrm{GdF}_{7}$, with the Gd element acting as the MRI contrast and barium $(\mathrm{Ba}, \mathrm{K}-$-edge $=37.4 \mathrm{keV})$ element as the $\mathrm{CT}$ contrast. Recent research has shown that ultrasmall nanoparticles can be rapidly cleared through the renal system; however, one of the main limitations of ultrasmall nanoparticles is the lack of accumulation at the tumor lesion sites. In order to circumvent this issue, as well as to specifically target tumor cell surface, Feng and colleagues produced epidermal growth factor receptor (EGFR)-targeted peptide-functionalized $\mathrm{Ba}_{2} \mathrm{GdF}_{7}$ nanoparticles (pEGFR-targeted $\mathrm{Ba}_{2} \mathrm{GdF}_{7} \mathrm{NPs}$ ). ${ }^{86}$ EGFR 
was proposed as the target for non-small cell lung cancer cells as it is overexpressed in over $70 \%$ of those affected. $\mathrm{Ba}_{2} \mathrm{GdF}_{7}$ nanoparticles were surface-functionalized with an EGFR-seeking peptide to facilitate targeted delivery of contrast media to the tumor location, resulting in positive tumor targeting MR/CT dual-mode imaging. In vitro testing was conducted using A549 lung carcinoma cells incubated with pEGFR-targeted $\mathrm{Ba}_{2} \mathrm{GdF}_{7}$ NPs, displaying high affinity. Additionally, pEGFR-targeted $\mathrm{Ba}_{2} \mathrm{GdF}_{7} \mathrm{NPs}$ were compared with a clinical MR contrast agent, displaying longitudinal relaxivity that is nearly double. When comparing pEGFR-targeted $\mathrm{Ba}_{2} \mathrm{GdF}_{7}$ NPs with clinical CT contrast agent (Omnipaque), it was discovered that to achieve the same contrast enhancement of $200 \mathrm{HU}$, pEGFR-targeted $\mathrm{Ba}_{2} \mathrm{GdF}_{7}$ NPs only needed to be a quarter as concentrated as Omnipaque. Finally, xenograft A549 lung tumor mouse model was used for assessment of the overall efficacy, as well as pharmacokinetics and toxicity of the pEGFR-targeted $\mathrm{Ba}_{2} \mathrm{GdF}_{7} \mathrm{NPs}$. It was found that with increasing time post-injection the contrast enhancement in both MRI and $\mathrm{CT}$ imaging increased, further confirming that pEGFR-targeted $\mathrm{Ba}_{2} \mathrm{GdF}_{7} \mathrm{NPs}$ are specifically accumulated and detained in tumor tissues. Furthermore, the size of pEGFRtargeted $\mathrm{Ba}_{2} \mathrm{GdF}_{7} \mathrm{NPs}$ allowed for efficient elimination from the biological system through the renal pathway, which helps to lower potential toxicity. Overall, this was proven to be a promising nanomaterial in dual-mode imaging, utilizing the advantages of both MRI and CT, as well as functional diversity of peptides, to be applied towards directed and wellrounded tumor diagnosis and therapy assessment. ${ }^{86}$

Similar nanoparticles containing gadolinium and barium ions, $\mathrm{BaGdF}_{5} \mathrm{NPs}_{\text {, were }}$ investigated by Wang et al. for targeted dual-mode imaging of angiogenic tumor vasculature. ${ }^{87}$ The surface of the nanoparticles was modified with a $\alpha_{\mathrm{v}} \beta_{3}$-targeting tri 
peptide, RGD. This allowed for targeting of $\alpha_{v} \beta_{3}$, the integrin adhesion molecule that is universally overexpressed in the endothelial cells of angiogenic tumor vasculature. Even at high concentrations, RGD-PEG-BaGdF 5 NPs demonstrated negligible cytotoxicity when tested against HUVEC cells in vitro. In vivo testing of RGD-PEG-BaGdF 5 NPs did not result in any apparent histopathological abnormalities or lesions in the heart, liver, spleen, lungs, or kidneys, further confirming good biocompatibility. When testing the ability of

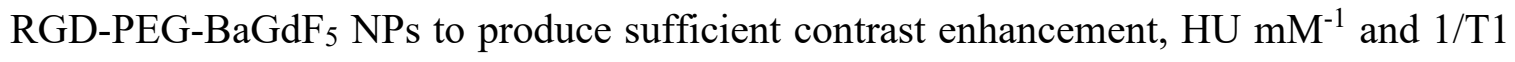
values were compared to those of iohexol and Gd-DTPA, respectively. The slope of the linear increase of $\mathrm{HU}$ in relation to RGD-PEG-BaGdF 5 NPs concentration was calculated to be $5.626 \mathrm{HU} \mathrm{mM}^{-1}$, which is higher than that of iohexol at $3.799 \mathrm{HU} \mathrm{mM}^{-1}$. Similarly, the $r_{l}$ value of RGD-PEG-BaGdF 5 NPs was calculated to be $1.584 \mathrm{mM}^{-1} \mathrm{~s}^{-1}$ and compared to $4.255 \mathrm{mM}^{-1} \mathrm{~s}^{-1}$ of Gd-DTPA, demonstrating MR T1 contrast performance, albeit weaker than that of Gd-DTPA. Dual-modal contrast performance and tumor vasculature-targeting was assessed in vivo, showing highest contrast enhancement at $0.5 \mathrm{hr}$ time-point for both $\mathrm{CT}$ and MR imaging. Furthermore, RGD-PEG-BaGdF5 NPs demonstrated highest enrichment in the reticuloendothelial system-rich organs, specifically accumulating in the tumor sites within $3 \mathrm{hrs}{ }^{87}$

\subsubsection{Aptamer-targeted contrast}

Aptamers are selected through a process of SELEX and are single-stranded oligonucleotides with propensity to bind their targets with high affinity and selectivity. They offer several advantages over other commonly used targeting ligands that can be utilized in the formulation of contrast agent for MRI and CT imaging. These advantages are based on their small size, non-immunogenic nature, tight and specific target binding, 
and ease of synthesis and handling. ${ }^{88-92}$ As above, Table 1.4 highlights some of the important aspects that were highlighted in the research described in this section. These can be used as recommendations for important elements of testing in the future development of aptamer-targeted contrast agents. 
Table 1.4: Summary of the recently developed aptamer-targeted contrast agents for applications in MR and CT imaging of various molecular markers of disease.

\begin{tabular}{|c|c|c|c|c|c|c|c|c|}
\hline $\begin{array}{r}\text { Aptamer targeted } \\
\text { contrast }\end{array}$ & Imaging target & $\begin{array}{l}\text { Aptamer } \\
\text { name }\end{array}$ & $\begin{array}{c}\text { Aptamer } \\
\text { length } \\
\text { (bases) }\end{array}$ & Modality & $\begin{array}{l}\text { Contrast } \\
\text { material }\end{array}$ & $\begin{array}{l}\text { Injected } \\
\text { dose }\end{array}$ & $\begin{array}{l}\text { Circulation } \\
\text { half-life }\end{array}$ & Ref \\
\hline $\begin{array}{r}A S 1411-G 2 \\
(D T P A-G d)-S S-P R\end{array}$ & Nucleolin & AS1411 & 28 & MRI & $\begin{array}{l}\text { Gd(III)- } \\
\text { DTPA }\end{array}$ & $\begin{array}{c}0.1 \\
\mathrm{mmol} \\
\mathrm{Gd} / \mathrm{kg}\end{array}$ & N/A & {$\left[{ }^{88}\right]$} \\
\hline$G_{m} L s$ & Tenascin-C & GBI-10 & 34 & MRI & $\begin{array}{c}\text { Gd(III)- } \\
\text { DTPA- } \\
\text { BSA }\end{array}$ & $\mathrm{N} / \mathrm{A}$ & $\mathrm{N} / \mathrm{A}$ & {$\left[{ }^{89}\right]$} \\
\hline M17-SPIONs & $\begin{array}{c}\text { Matrix } \\
\text { metalloproteinase } 14\end{array}$ & M17 & 40 & MRI & SPIONs & $\mathrm{N} / \mathrm{A}$ & $\mathrm{N} / \mathrm{A}$ & {$\left[{ }^{90}\right]$} \\
\hline Den-Apt1 & Endoglin & $\begin{array}{l}\text { Endoglin- } \\
\text { targeting } \\
\text { apt }\end{array}$ & 38 & MRI & $\begin{array}{l}\text { Gd(III)- } \\
\text { DTPA }\end{array}$ & $\begin{array}{c}0.05 \\
\mathrm{mmol} \\
\mathrm{Gd} / \mathrm{kg}\end{array}$ & $\mathrm{N} / \mathrm{A}$ & {$\left[{ }^{91}\right]$} \\
\hline $\begin{array}{r}m E N D- \\
\mathrm{Fe}_{3} \mathrm{O}_{4} @ C M C S\end{array}$ & Mouse endoglin & eEND & 60 & MRI & SPIONs & $\begin{array}{c}0.05 \\
\mathrm{mmol} \\
\mathrm{Fe} / \mathrm{kg} \\
\end{array}$ & $\mathrm{N} / \mathrm{A}$ & {$\left[{ }^{92}\right]$} \\
\hline $\begin{array}{r}\text { Wy5a-SPIO/Dtxl- } \\
\text { NPS }\end{array}$ & $\begin{array}{l}\text { Castration-resistant } \\
\text { prostate cancer cells }\end{array}$ & Wy5a & 57 & MRI & SPIONs & $\begin{array}{c}10 \mathrm{mg} \\
\mathrm{Dtxl} / \mathrm{kg}\end{array}$ & $\begin{array}{c}\text { Controlled } \\
\text { drug release } \\
\text { for up to } 5 \\
\text { days }\end{array}$ & {$\left[{ }^{93}\right]$} \\
\hline$A u-T_{i} O_{2}-A-T P P$ & Nucleolin & AS1411 & 28 & $\mathrm{CT}$ & Gold & $\begin{array}{l}10 \mathrm{mg} \\
\mathrm{Gd} / \mathrm{kg}\end{array}$ & $4.71 \mathrm{hrs}$ & {$\left[{ }^{94}\right]$} \\
\hline $\begin{array}{r}\text { Apt-PEG- } \\
\text { AuPAMAM-CUR }\end{array}$ & Mucin-1 & MUC-1 & 35 & $\mathrm{CT}$ & Gold & $\begin{array}{c}2 \mathrm{mg} \\
\mathrm{CUR} / \mathrm{kg}\end{array}$ & $\mathrm{N} / \mathrm{A}$ & {$\left[{ }^{95}\right]$} \\
\hline $\begin{array}{r}\text { Apt-ALGDG2- } \\
\text { Iohexol }\end{array}$ & Nucleolin & AS1411 & 26 & $\mathrm{CT}$ & Iohexol & $1.6 \mu \mathrm{M}$ & N/A & {$\left[{ }^{96}\right]$} \\
\hline
\end{tabular}


$\mathrm{Zu}$ and colleagues have recently developed a macromolecular MRI contrast agent using polyrotaxanes, which are cyclic molecules threaded onto a polymer "axle" and capped with bulky endcaps ${ }^{88}$ One of the advantages of polyrotaxanes is their prolonged blood circulation, as they are not as readily internalized by macrophages as small molecules. The proposed structure consisted of disulfide bonds located at the terminal ends of the molecule. These bonds are readily cleaved in the cytoplasm, thus resulting in dethreading of the overall structure into smaller subunits and subsequent elimination. The system was modified with an aptamer (AS1411) that is specific to a highly overexpressed multifunctional surface-protein, nucleolin. Testing revealed that longitudinal relaxivity of the novel complex was double that of the clinically used $\mathrm{Gd}^{3+}$-DTPA contrast agent. Additionally, AS1411-G2(DTPA-Gd)-SS-PR demonstrated superior biocompatibility, not eliciting accumulation-associated toxicity when it was tested against HUVAC. Targeting ability of AS1411, designed to enhance MRI performance, was confirmed in vitro and in vivo using MCF-7 cells overexpressing nucleolin on the surface and a subcutaneous tumorbearing mouse model, respectively. Faster accumulation at the tumor site and longer contrast imaging time confirmed that this formulation may be a good candidate for clinical applications for tumor imaging. ${ }^{88}$

In another example, a DNA aptamer (GBI-10) that specifically binds tenascin-C (TN-C), an extracellular glycoprotein highly expressed on the surface of solid tumor tissues in the brain, breast, uterus, ovaries, prostate, pancreas, colon, stomach, mouth, larynx, and many others was utilized. In order to improve the binding of GBI-10, as well as enhance contrast-associated relaxivity in MRI, Zhang et al. prepared gadolinium-loaded liposomes that were surface-modified with iso-nucleoside-modified GBI-10 (GBI-10m), $\mathrm{G}_{\mathrm{m}} \mathrm{Ls} .{ }^{89} \mathrm{In}$ 
order to further improve relaxivity of this novel contrast formulation, paramagnetic lipid Gd-DTPA-BSA was incorporated into the membrane of the liposomes. As expected, in T1weighted MR images, $\mathrm{G}_{\mathrm{m}} \mathrm{Ls}$ produced higher signal intensity than that of the controls or Gd-DTPA at similar concentrations. Targeting abilities were assessed in vitro using MDAMB-435s cells, which are human breast duct cells overexpressing TN-C, and fluorescently modified $\mathrm{G}_{\mathrm{m}} \mathrm{Ls}$ for simplicity of visualization. Fluorescence intensity of the cells incubated with $\mathrm{G}_{\mathrm{m}} \mathrm{Ls}$ was two times higher than that of control incubations. Additionally, cellular uptake of $\mathrm{G}_{\mathrm{m}} \mathrm{Ls}$ was observed, confirming that TN-C (+) cells were susceptible to $\mathrm{G}_{\mathrm{m}} \mathrm{Ls}$ internalization. It was evident that the presence of the aptamer in the final liposomal formulation, $\mathrm{G}_{\mathrm{m}} \mathrm{Ls}$, resulted in higher targeting thereby improving specificity. In addition, the liposomal preparation itself allowed for increased contrast production based on the presence of higher Gd concentrations. Additionally, choosing an appropriate aptamer resulted not only in targeting of the surface-expressed receptors but also facilitated liposomal uptake into the tumor. ${ }^{89}$

Matrix metalloproteinase 14 (MMP14) is a cellular marker that can be used to detect pancreatic cancer, hepatocellular carcinoma, lung carcinomas, gastrointestinal carcinomas, breast carcinomas, gliomas, and cervical carcinomas. The Huan group was able to select a DNA aptamer, M17, using MMP14-transfected 293T cells..$^{90}$ In vivo fluorescence imaging, utilizing MIA PaCa-2 tumor-bearing $\mathrm{BALB} / \mathrm{c}$ mice, was used to confirm that M17 is able to specifically recognize MMP14-positive cells. They subsequently utilized M17 in surface-functionalization of magnetic nanoparticles $\left(\mathrm{Fe}_{3} \mathrm{O}_{4}\right)$ for MRI imaging. T2 values of the control cell, as well as control aptamer incubations were compared to those of M17-SPIONs and MMP14-transfected 293T incubation, with values 
significantly higher for the latter. Overall, targeted delivery resulted in high specificity and high affinity of SPIONs towards MMP14 marker, confirming that this formulation is a promising targeting agent with potential to be used for targeted diagnosis and treatment of MMP14-positive cancers. ${ }^{90}$

It is commonly accepted that early cancer detection is crucial for optimized therapeutic response. While hepatocellular carcinoma (HCC) is readily diagnosed in its late stage with the help of MRI and CT imaging, detection of lesions smaller than $2 \mathrm{~cm}$, associated with early stages of this cancer, is $47 \%$ or less. Yan and colleagues proposed an aptamer-functionalized formulation in order to improve the detection of these tiny HCC lesions, thereby improving the prognosis of $\mathrm{HCC}$ patients. ${ }^{91}$ The final contrast nanoprobe used endoglin targeting in order to image small-volume HCCs with high sensitivity and specificity. Endoglin is a marker that is overexpressed on the vascular endothelium of solid tumors. The selected endoglin-specific aptamer was conjugated to $\mathrm{Gd}^{3+}$-DTPA, as well as near-infrared fluorophore IR783 on a G5 dendrimer scaffold, denoted Den-Apt1 (Figure 1.5 A). MRI phantom imaging was used to confirm the capability of Den-Apt1 to produce contrast, demonstrating linear correlation between its increasing concentration and relaxation rates in T1-weighted imaging. The fluorescent component of the probe was used to confirm targeting efficiency of Den-Apt1 towards HCC cell lines in vitro, displaying significantly higher intracellular signal 2 hours post treatment when compared the untargeted control. In vivo studies were conducted using nude mice bearing orthotopic hepatic humor xenografts and Den-Apt1 as well as Den-PEG injected through an i.v. When comparing the contrast signal of Den-Apt1 with that of $\mathrm{Gd}^{3+}$-DTPA, the $\mathrm{T} 1$ signal associated with Den-Apt1 was substantially higher at the tumor margins after 30 mins 
(Figure $1.5 \mathrm{~B}$ ). After 1 and $24 \mathrm{~h}$ post injections, the tumor area became more enhanced, owing its contrast to the endoglin-mediated tumor uptake of Den-Apt1. Finally, ex vivo optical imaging was used to further verify the biodistribution of Den-Apt1, confirming its accumulation at the site of HCC (Figure 1.5 B). Overall, this nanoprobe allows for effective delineation of the tumor surface, holding promise towards guided tiny HCC surgery. ${ }^{91}$
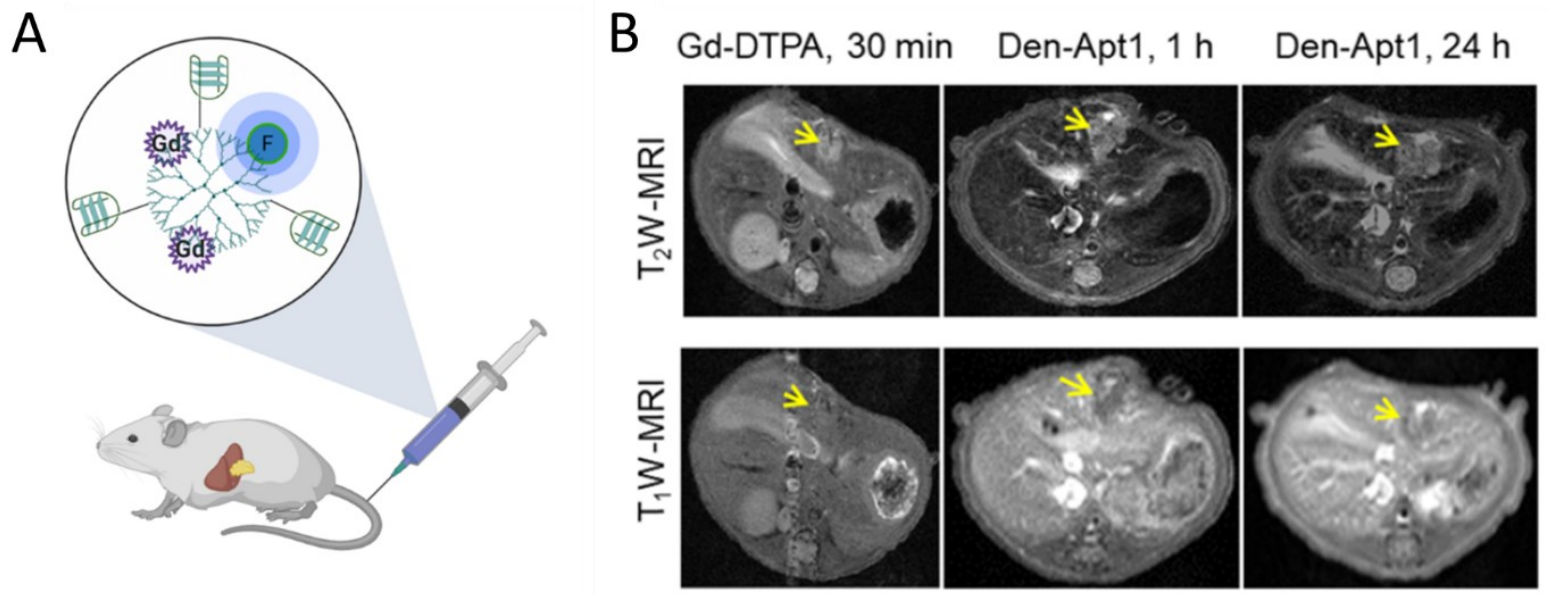

Figure 1.5: (A) Illustration of the dendrimer system, labelled with Gd chelate, a NIR fluorophore, and the endoglin-targeting aptamer, made in BioRender. (B) Sample T1- and T2-weighted MR imaging of hepatocellular carcinoma xenografts at $1 \mathrm{hr}$ (Middle) and $24 \mathrm{hrs}$ post treatment with Den-Apt1 (Right) compared to conventional agent Gd-DTPA (Left). Adapted with permission from American Chemical Society. ${ }^{91}$

Zhong et al. proposed a different variation of endoglin-targeting MRI contrast agent. Their group selected an aptamer that specifically bound to mouse endoglin (mEND) on murine neovascular endothelial cells. The final nanoprobe consisted of the superparamagnetic $\mathrm{Fe}_{3} \mathrm{O}_{4}$ producing $\mathrm{T} 2$ contrast, and carboxymethyl chitosan (CMCS) as the wrapping agent to ensure biocompatibility and reduced toxicity (as mEND$\left.\mathrm{Fe}_{3} \mathrm{O}_{4} @ \mathrm{CMCS}\right) .{ }^{92}$ As previously, the mEND aptamer was to serve as the recognition 
molecule, ensuring that the $\mathrm{Fe}_{3} \mathrm{O}_{4} @ \mathrm{CMCS}$ contrast would be specifically delivered towards the target. The final $87 \mathrm{~nm}$ nanoprobe was tested to confirm targeting ability, as well as contrast enhancement. In vitro experiments confirmed that mEND-Fe $\mathrm{O}_{4} @ \mathrm{CMCS}$ nanoprobes could specifically bind to vascular endothelial cells expressing endoglin with superior targeting ability as compared to controls. When comparing MR signals, mEND$\mathrm{Fe}_{3} \mathrm{O}_{4} @$ CMCS produced significantly enhanced signal in T2-weighted imaging at the same concentration as unmodified $\mathrm{Fe}_{3} \mathrm{O}_{4}$. In vivo experiments, using $\mathrm{HCC}$-bearing mice injected with mEND-Fe $\mathrm{O}_{4} @ \mathrm{CMCS}$, confirmed that this nanoprobe is able to produce enhanced contrast at the tumor site. This confirms that targeting of MR contrast agent can improve specific imaging of HCC tumors. ${ }^{92}$

Fang et al. recently reported an aptamer-conjugated formulation using multifunctional polymeric nanoparticles for applications in advanced prostate cancertargeted MRI and controlled drug delivery. An aptamer, Wy5a, was selected through cellSELEX to specifically bind castration-resistant prostate cancer cells (PC-3). Self-assembly method, using a pre-functionalized triblock copolymer (PLGA-b-PEG-b-Wy5a), was utilized to produce "core-shell" nanoparticles that encapsulated superparamagnetic iron oxide nanoparticles (SPION) and docetaxel (Dtxl). SPIO was used to produce negative contrast in T2-weighted scans, while Dtxl, a chemotherapeutic drug for CRPC, was used for controlled treatment. Initial assessment of contrast enhancement capabilities showed that encapsulation of SPION did not reduce the associated magnetization effects. In fact, their clustering resulted in significant $\mathrm{T} 2$ relaxation shortening with relaxivity values approximately 2 -fold higher than the commercially available Resovit. In vitro testing confirmed that Wy5a-SPIO/Dtxl-NPs are able to produce significantly enhanced negative 
contrast associated with selective internalization achieved through Wy5a targeting PC-3 cells. Wy5a-SPIO/Dtxl-NPs also demonstrated controlled drug release with significantly higher cytotoxicity when compared to non-targeted counterpart or Dtxl alone; IC50 was 1.42-fold and 1.27-fold lower than those of non-targeted counterpart and Dtxl, respectively. In vivo antitumor efficacy was also confirmed through significant tumor regression achieved in the presence of Wy5a-SPIO/Dtxl-NPs. It is evident that multifunctional formulations like this one are a promising class of targeted drug and contrast delivery systems. ${ }^{93}$

A handful of recent examples are centered around the use of aptamers as targeting moieties in CT imaging. These formulations are developed based on the previously underlined principle of high atomic number elements, such as gold and iodine, experiencing high X-ray attenuation. One such complex was devised by Cao et al., using a combination of gold nanocrystals grown on $\mathrm{TiO}_{2}$ nanosheets and targeting elements, including mitochondria-targeted triphenylphosphine (TPP) and AS1411 aptamer. ${ }^{94}$ These components were used to achieve multifunctionality in therapeutic and imaging applications. Specifically, incorporating noble metals like gold has been shown to improve the quantum yield of reactive oxygen species in sonodynamic therapy. Conveniently, gold can then be used to trace the $\mathrm{TiO}_{2}$-based nano-agents and assess their utility as sonosensitizers. In this case, aforementioned AS1411 aptamer was used for targeting of the cancer cell-membrane through nucleolin, while TPP was used to assist in delivery to and accumulation in the mitochondria. In the context of sonodynamic therapy, $\mathrm{Au}-\mathrm{TiO}_{2}-\mathrm{A}-\mathrm{TPP}$ was tested both in vitro and in vivo, resulting in complete tumor growth suppression. For CT monitoring, increasing concentrations of $\mathrm{Au}-\mathrm{TiO}_{2}-\mathrm{A}-\mathrm{TPP}$ exhibited a linear 
relationship to X-ray attenuation values (CT value, $\mathrm{HU}$ ) and increasingly observable brightening contrast. Bright contrast was observed at the location of the tumor when 10 $\mathrm{mg} / \mathrm{kg}$ of $\mathrm{Au}-\mathrm{TiO}_{2}-\mathrm{A}-\mathrm{TPP}$ was administered through an i.v. to MCF-7 tumor-bearing female $\mathrm{BALB} / \mathrm{c}$ nude mice, confirming the ability of $\mathrm{Au}-\mathrm{TiO}_{2}$-A-TPP to selectively accumulate due to targeting. This type of multifunctional formulation can be reproduced with different targeting elements for applicability towards other types of cancer cells. ${ }^{94}$

In a different study, Alibolandia and colleagues investigated the use of poly(amidoamine) PAMAM G5 dendrimers for encapsulation of curcumin and GNPs (a formulation the foundation of which is similar to that reported by Yan et al. $)^{91}$ as a targeted multifunctional theranostic nanoplatform. ${ }^{95}$ Tumor tissue targeting was achieved through the use of MUC-1 aptamer, which selectively binds to mucin-1 receptors overexpressed during angiogenesis and tumorigenesis in a number of cancers, including colorectal, breast, lymphocytic leukemia, adrenal cell and prostate carcinomas. Curcumin is a naturally occurring polyphenol which provides anti-inflammatory and anti-neoplastic effects; however, its poor aqueous solubility, low bioavailability, and high blood clearance require its delivery in an appropriate vehicle. This study evaluated the ability of Apt-PEGAuPAMAM-CUR to selectively target mucin-1, thereby delivering curcumin while synchronously providing CT contrast. Both in vitro, using MUC-1 positive cell lines (HT29 and C26), and in vivo, using subcutaneously developed colorectal adenocarcinoma (C26 cell line) in BALB/C mice, studies were utilized to investigate targeting efficiency and cellular toxicity of Apt-PEG-AuPAMAM-CUR. When compared to controls, treatments with Apt-PEG-AuPAMAM-CUR resulted in selective targeting, which elicited significantly higher cytotoxicity. Attenuation capabilities of Apt-PEG-AuPAMAM-CUR 
were assessed in vivo after i.v. administration. Highly localized contrast enhancement was observed and attributed to aptamer-targeted delivery of Apt-PEG-AuPAMAM-CUR to the tumor site. Through utilization of different targeting elements, this technology would allow for accurate diagnosis, specific delivery of therapeutic agents, and monitoring of therapeutic response of different adenocarcinomas. ${ }^{95}$

Mohammadzadeh and co-workers explored a different type of aptamer-decorated dendrimer as a nanocarrier for CT contrast material. ${ }^{96}$ Specifically, anionic linear globular dendrimer G2 (ALGDG2) was loaded with conventionally used CT contrast agent, iohexol, and surface-functionalized with the AS1411 aptamer. As above, AS1411 was used for targeting cancer cells and the main objectives were to decrease off-target toxicity and reduce dosage required for significant contrast enhancement. Off-target effects such as cytotoxicity and apoptosis were tested in vitro using nucleolin-positive human breast cancer cells, MCF-7 and HEK-293 as the control cell line. The overall observed trend showed that non-encapsulated iohexol and un-targeted ALGDG2-Iohexol, but not AptALGDG2-Iohexol, elicited cytotoxicity on HEK-293 cells after 72 hours. Further to that, Apt-ALGDG2-Iohexol produced a toxic effect on MCF-7 cells. This was supported when apoptosis was observed after treatment of HEK-293 cells with iohexol and ALGDG2Iohexol. Targeting efficiency was also confirmed, demonstrating significantly higher uptake of Apt-ALGDG2-Iohexol when compared to its untargeted counterparts. In vivo studies, using 4t1 tumor-bearing female BALB/c mice, were conducted to assess imaging ability and further confirm targeting efficiency. After i.v. administration of Apt-ALGDG2Iohexol into the tail vein, tumor sites were clearly visible in spiral CT imaging after 20 mins. Histopathological assessments were performed by harvesting kidney, spleen, liver, 
and tumor tissues, as well as blood, and no considerable toxicity was observed. This type of design opens doors for other applications in the field of cancer molecular imaging. ${ }^{96}$

Overall, it is expected that, in the future, there will be more ligands selected towards other targets and incorporated into formulations consisting of contrast elements. These formulations will likely not only be targeted, but also have the ability to be used as dualmode contrast or theranostics, facilitating confirmation of diagnosis through several different imaging modalities or allowing for integration of precise diagnostics and therapeutics, respectively. A number of controls are described within these studies and could be used as guidelines for future research. In vitro testing helps determine cytotoxicity and preliminary binding efficiency of the formulations. Here, it is important to test cell lines that display the target of interest and are indicative of the tissue of interest. In addition, off-target toxicity should be assessed through the use of cell lines that represent the suspected route of clearance. In vivo assessment should be conducted after distal injections using animals that can express human cell lines, while controls for these treatments can include contrast formulations that are either untargeted or modified with a scrambled sequence.

\subsection{Project goals}

It is evident that incorporation of a targeting ligand into currently existing contrast materials allows for overall higher contrast enhancement and targeted visualization of different components of disease states. Throughout the research outlined above, both peptides and aptamers demonstrated excellent targeting abilities, allowing for optimized biodistribution, while remaining non-immunogenic. Their superior selectivity results in contrast accumulation at the target of interest, thereby eliciting improved enhancement 
when compared to commercially available contrast media. Since each of the targeting ligands are selected specifically, off-target effects were shown to be limited.

The following chapters will discuss the development of fibrinogen aptamertargeted conjugates as proof-of-concept formulations for molecular imaging of vascular diseases which involve blood clotting. We hypothesized that, in order to streamline the development of these conjugates, we could utilize an aptamer that was previously selected for the fibrinogen monomer, structural elements of which remain predominantly unchanged during the polymerization process. In the initial stages of the project, the binding of fibrinogen aptamer to fibrin protein was extensively characterized in order to ensure that affinity and selectivity are not compromised. The final fibrinogen aptamer formulations were assessed for their ability to produce significant contrast enhancement. The goal of this assessment was to ensure that the composition of these contrast agents would allow for use of concentrations that are lower than ones required by the clinically available materials. It was proposed that targeting would result in high local concentrations at the location of the clot, which would have a three-fold advantageous effect. Selective accumulation of the contrast material would decrease non-specific distribution, which would allow for use of lower injection concentrations. Lowering the concentration would in-turn lower the possibility of adverse effects and toxicity. In addition, selective accumulation at a specific site would allow for more precise diagnosis and effective followup. In the case of the aptamer-targeted gadolinium-based agent, we predicted that target binding would improve relaxivity rates, producing a more pronounced effect in T1weighted imaging. Through in vitro testing, however, unexpected behavior of this conjugate came to light, with blood clot characteristics, as well as the presence of a long 
aptamer strand, having a dominant effect on the performance of the paramagnetic ion. In the production of the $\mathrm{CT}$ agents, we postulated that we could push the limits by incorporating a large number of X-ray attenuating materials. As such, we expected that lower concentrations of both the iodine- and the gold nanoparticle-based formulations will be required to produce measurable enhancement. Lastly, as an alternative to incorporating clinically applicable materials to achieve targeted contrast accumulation, we produced core shell nanoparticles which were made up of elements that elicit contrast enhancement in both MRI and CT. We hypothesized that the iron core would allow for high-resolution T2weighted imaging in MRI, while the gold coating would be applicable towards CT imaging. This would, in turn, limit the need for unnecessary re-administration of contrast material when switching between two imaging modalities. Finally, keeping in mind that fibrinogen aptamer binds fibrinogen monomers, the clotting process was tested for any sign of interruptions to ensure that the presence of aptamer-guided contrast does not act as a thrombolytic, leading to excessive bleeding. This was especially important for scenarios where these formulations are used in the diagnosis of hemorrhagic strokes. We predicted that, since the selected fibrinogen aptamer candidate binds away from the areas of the protein which are directly involved in polymerization, the presence of fibrinogen aptamerbased contrast materials would not have an effect on clotting. 


\section{Chapter 2: Analysis Techniques}

\subsection{Statement of contribution}

This chapter was written by A Koudrina and provides descriptions of the working principles behind the methodological approach used in this research. These techniques were specifically chosen to accommodate the characterization and assessment of the novel targeted contrast media. 


\subsection{Aptamer characterization methods}

\subsubsection{Ultraviolet-visible spectroscopy}

For convenience of characterization, light is commonly described in terms of both waves and particles. When depicting a plane-polarized light wave, it is important to consider the two oscillating components, electric and magnetic fields. The electric field oscillates in the $x y$-plane, while the magnetic field is in the $x z$-plane, which means they are perpendicular to one another. This allows to define a wavelength $(\lambda)$, which is the crest-tocrest distance between wave peaks. Within an electromagnetic spectrum, visible spectrum occupies the wavelength range of $380-780 \mathrm{~nm}$, while ultraviolet spectrum is $10-400 \mathrm{~nm}$. With regards to energy, light can be represented as a particle, or a photon. That is, photons possess energy $(E)$ that is inversely proportional to the wavelength, which is depicted by equation 2.1,

$$
E=h v \quad 2.1
$$

where $h$ is Planck's constant and equals to $6.626 \times 10^{-34} \mathrm{~J} \cdot \mathrm{s}$, and $v$ is the frequency, denoting the number of oscillations within the light wave. Absorbing visible or ultraviolet radiation promotes molecules into excitation states due to electron transfer to higher orbitals. It is well understood that light absorbance $(A)$ is directly proportional to the concentration of a light-absorbing species and can, therefore, be used to determine said concentration. This can be described by Beer-Lambert's law (equation 2.2),

$$
A=\log _{10}\left(\frac{I_{0}}{I}\right)=\varepsilon l c \quad 2.2
$$

where $I_{0}$ is the initial light intensity and $I$ is the transmitted light intensity, which is related to $c$, which is the concentration of the sample, $l$ which is the pathlength, and $\varepsilon$ which is molar absorptivity, otherwise known as molar extinction coefficient. Molar absorptivity is 
specific to the entity that is measured. Spectrophotometry is a commonly used technique which allows for measurement of these parameters. ${ }^{97}$

Specifically, ultraviolet-visible (UV-Vis) spectrometry allows for assessment of species that absorb energy within the wavelength range of 100-800 $\mathrm{nm}$. Chromophores are molecular structures responsible for light absorbance at a specific frequency. In terms of oligonucleotides, purines and pyrimidines experience an absorption maximum at an average of $260 \mathrm{~nm}$. Each base is represented by its respective molar extinction coefficient, and, therefore, the absorbance in this region is the sum of each individual absorbances of the oligo-bases present in solution. Proteins can also be assayed in the ultraviolet region, and specifically at $280 \mathrm{~nm}$, as they commonly possess aromatic groups with an absorbance maximum at this specific wavelength..$^{97,98}$

\subsubsection{Circular dichroism}

The complex three-dimensional shapes of aptamers are dictated by the presence of a variety of structures like stems, loops, bulges, hairpins, pseudoknots, triplexes, or quadruplexes. In a similar manner, target binding occurs based on not only structural compatibility, but also stacking or aromatic rings, electrostatic and van der Waals interactions, hydrogen bonding, or some combination thereof. ${ }^{11}$ In order to assess the minute structural changes that may occur upon ligand binding, a sensitive technique such as circular dichroism (CD) has been employed. $\mathrm{CD}$ is an optical technique that uses the general principle of variance in absorption of left- and right- circularly polarized light by

optically active chiral molecules. ${ }^{99,100} \mathrm{CD}$ spectra can be broken down into two regions, encompassing wavelengths between $190 \mathrm{~nm}$ and $350 \mathrm{~nm}$, with approximately 190-250 nm representing the far-UV region and approximately $250-350 \mathrm{~nm}$ representing the near-UV 
region. Fortunately, in the assessment of aptamer binding to proteins, the secondary structures of the two do not typically interfere with one another. That is, the $\alpha$-helix, $\beta$ sheets, coiled or uncoiled structures within the protein will be measured within the far-UV region. Meanwhile, the exciton coupling achieved through base stacking in the formation of a secondary structure of oligonucleotides gives rise to measurable signals in the nearUV region. ${ }^{101}$

The most common conformation of DNA is B-form, characterized by two distinct peaks, with a positive long wavelength band between $260 \mathrm{~nm}$ and $280 \mathrm{~nm}$ and a negative peak between $240 \mathrm{~nm}$ and $260 \mathrm{~nm}$. It is important to note that, intrinsically, nucleobases are planar, which means that observable asymmetry arises due to the sugars in the sugarphosphate backbone. Nucleobases have a structure that is indicative of a chromophore, based on the $\pi-\pi^{*}$ transitions of the bases upon UV light exposure, with position and the amplitude of the associated peaks dictated by the sequence of DNA. ${ }^{100,102,103}$ The shifts in the position and amplitude within these peaks largely depends on the base composition of the strand. While other conformations, including A- and Z-DNA, as well as distinctly different guanine-quadruplexes, are possible, they were not explored in this work. ${ }^{99,100}$ It has been reported that aptamer-target binding can be characterized through the monitoring of changes of peak characteristics upon variation of the target concentration, which can then be used in determining the binding constant. ${ }^{100,102}$

\subsubsection{Isothermal titration calorimetry}

Another useful technique in determination of the binding constant and the thermodynamic parameters of the binding is isothermal titration calorimetry (ITC). This is a sensitive, label-free technique, which measures the energetics of biochemical reactions, 
particularly the heat taken up from the surroundings (endothermic processes) or released to the surroundings (exothermic processes). Specifically, the reaction is triggered by titrating the reactant, thereby changing the composition of the sample. As such, the rate at which heat is exchanged with the surroundings, or the thermodynamic observable, is equal to the enthalpy of the reaction and the extent to which it has occurred. ITC offers a number of major advantages, including the ability to be performed with components that are spectroscopically silent, ability to assess opaque, turbid, or heterogeneous solutions, all of which can be done in a range of biologically relevant conditions. The measurements are set up in such a way that the calorimeter measurement cell is kept at a chosen constant temperature (isothermal). During the process of chemical titration, the power applied to the control heater is either increased or reduced, based on the type of interaction that occurs. Raw data is, therefore, presented as the power applied to the control heater as a function of time $(\mu \mathrm{cal} / \mathrm{sec}$ or $\mu \mathrm{J} / \mathrm{sec}){ }^{104,105}$

The binding contrast, as well as other thermodynamic parameters, can then be calculated, using equation 2.3 .

$$
Q=V \times \Delta H \times\left[[L]+\frac{1+[M] \times n \times K-\sqrt{(1+[M] \times n \times K-[L] \times K)^{2}+4 \times K \times[L]}}{2 \times K}\right]
$$

where, $\Delta H$ is the enthalpy of the binding, $K$ is the binding constant, $n$ is the number of binding sites, with other variables including $V$, which is the volume of the cell, $[L]$, which is the total ligand concentration, and $[M]$, which is the protein concentration. ${ }^{104,105}$

\subsubsection{Microscale thermophoresis}

Microscale thermophoresis (MST) is a sensitive technique that allows for quantification of biomolecular interactions that are free in solution. As implied by the name, this technique relies on an effect referred to as thermophoresis, otherwise known as 
the Soret effect, or thermodiffusion, which describes a directed movement of molecules along a temperature gradient. This movement is dependent on various parameters, such as size, charge, hydration shell or conformation. In MST, the movement of fluorescent molecules through microscopic temperature gradient in microliter volumes allows for precise assessment of binding events. This is recorded based on the depletion or accumulation of molecules in regions with elevated temperatures, which shows dependence on the interface between the molecule and the solvent. This interface is affected by events such as ligand-binding, augmenting the overall size, charge, and hydration shell of the target molecule. Importantly, even in the absence of a dramatic change in size and charge, which could be associated large relative difference between the ligand and the protein, MST is able to detect the binding event based on the changes in molecules solvation entropy. ${ }^{106,107}$

When assessing binding events, MST uses glass capillaries which consist of the ligand and the protein, prepared in a range of concentrations. The infrared (IR) laser, used to produce the precise microscopic temperature gradient, is coupled with an excitation light, which allows for fluorescence monitoring of thermophoretic movement of the fluorophores (Figure 2.1 A). The recorded fluorescence time trace is made up of a number of components (Figure 2.1 B). First, fluorescence of the evenly distributed and freely diffusing components is monitored. The IR laser is then activated to initiate thermophoresis, with unbound components migrating considerably faster than those in a ligand-target complex, which alters the movement. This movement is recorded until a steady state is achieved. Disruption of this steady state, through deactivation of the IR laser, results in further movement which is geared towards re-establishment of homogeneity 
within the solution. Upon the increasing presence of the target, which correlates to increased number of binding events, a change in thermophoresis is observed. This change is visualized though the shift of the plateau within the binding isotherm (Figure 2.1 C). ${ }^{106,107}$ For the purposes of this research, the binding partner pair consisted of the fibrin protein and Cy5-modified fibrinogen aptamer.

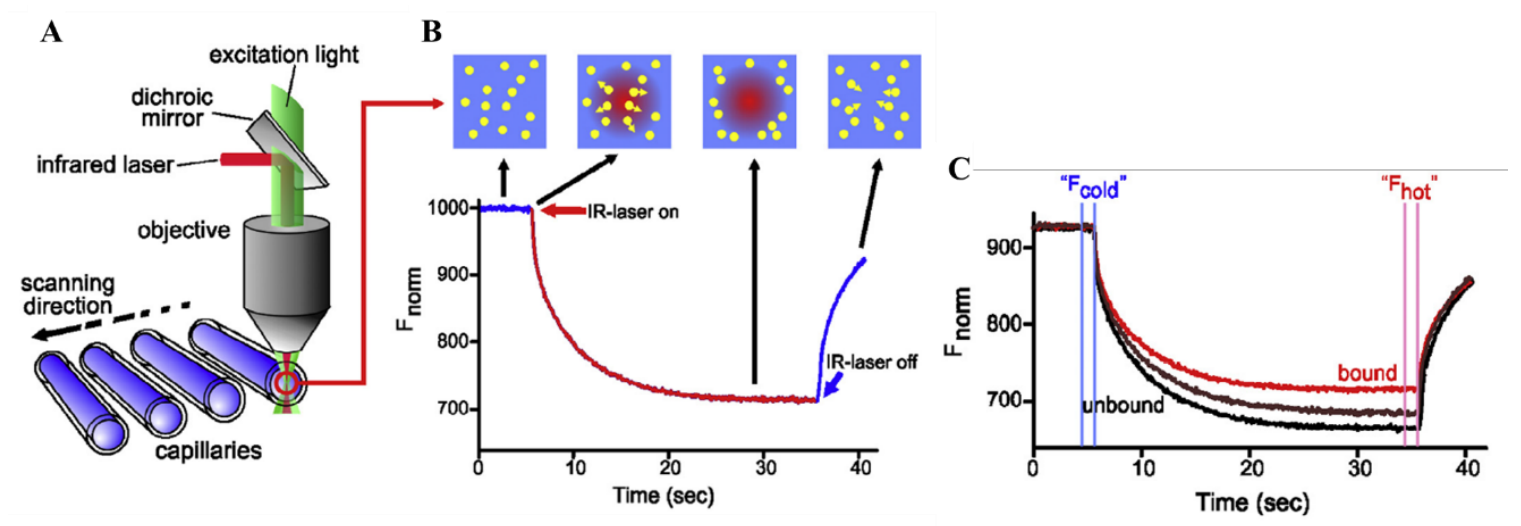

Figure 2.1: (A) MST instrumental set-up, demonstrating the simultaneous induction of a temperature gradient and excitation of the fluorophore located within the capillaries, which are scanned in sequence; (B) A sample fluorescence time-trace, indicating IR laser activation and de-activation, with thermophoresis dependent depletion of fluorophores; (C) A sample binding isotherm with ligandtarget complex demonstrating a higher fluorescence plateau than the unbound species. Adapted with permission from Elsevier (Open Access). ${ }^{107}$

\subsection{Aptamer conjugation methods}

\subsubsection{Maleimide-Thiol reaction}

To facilitate the conjugation of the aptamer with the macrocyclic bifunctional chelators, used for complexing of the gadolinium ion in the aptamer-MRI contrast agents, Michael-addition reaction was utilized. This is a reaction of a thiol to a maleimide which 
is commonly used for bioconjugation of drugs to a macromolecule. ${ }^{108} \mathrm{~A}$ similar approach was explored by Gijs et al. in the production of 1,4,7-triazanonane-1,4,7-triyl)triacetic acid (NOTA)-conjugated oligonucleotides, which were subsequently radiolabelled with gallium-68 for molecular imaging of epidermal growth factor receptor 2 (HER2). ${ }^{109}$ The aptamer was initially functionalized at the 5 '-end with a hexyldisulfide group, which generated a singular binding site for the incorporation of the chelator. This controls for the position and the precision of subsequent complexation, avoiding undesirable sideproducts. ${ }^{109}$ Noteworthy, while other single-step bioconjugation strategies exist, such as hexylamine-modified aptamer with isothiocyanatobenzyl chelator, it is unclear whether amino groups within the aptamer bases may become involved. ${ }^{14,110}$ Both $1,4,7,10$ tetraazacyclododecane-1,4,7,10-tetraacetic acid (DOTA) and NOTA-apt bioconjugates were produced in a two-step synthesis. First, the hexyldisulfide group was reduced with the help of dithiothreitol, a redox agent, to yield a free reactive thiol group. ${ }^{111}$ Maleimidomonoamide-DOTA/NOTA macrocyclics were subsequently reacted in excess to ensure full incorporation. ${ }^{109}$ Equimolar proportion of the DOTA/NOTA-apt conjugates and Gd(III) were mixed together to allow for assessment of loading efficiency using xylenol orange test (discussed in section 2.4.1). ${ }^{14}$

\subsubsection{Gold surface-functionalization}

Functionalization of gold and gold-coated nanoparticle surfaces is well established and endows many desirable characteristics to these nanoscale materials. One of the most convenient methods of attainment of self-assembled monolayers (SAM) is through the use of thiol-modified entities. In the production of both gold nanoparticles, and gold-coated nanoparticles, chemical reduction of transition metal salt is utilized in the presence of a 
stabilizing agent is utilized. Functionalization is achieved through the sulfhydryl (SH) functional group of the thiol, resulting in a robust covalent bond between gold and sulfur. Using a monothiol allows for controlled deposition of the thiolated ligand. With this in mind, all aptamer-functionalized nanoparticles used in this research were produced as 5'thiol modified strands. ${ }^{12}$ The disulfide bridge was cleaved with dithiothreitol (DTT), a reducing agent that is itself a thiol, in an intramolecular reaction through an exchange of the thiolate anion. This yielded a free sulfhydryl group, and a stable cyclic disulfide byproduct. ${ }^{111,113}$ This product can, therefore, be directly reacted with the gold surface immediately following the cleavage to produce a SAM. ${ }^{112}$

\subsection{Conjugate characterization}

\subsubsection{Xylenol orange test}

In the production of the Gd(III)-DOTA/NOTA-apt contrast material, the final formulation was washed and the flow-through was assessed for its free Gd(III) content, relating it back to loading efficiency. Xylenol orange, a complexometric dye, is a common indicator used to assess the concentration of lanthanide(III) ions. In this case, complexation with a free $\mathrm{Gd}(\mathrm{III})$ ion mimics a $\mathrm{pH}$ change, due to the occurrence of coordination through the iminodiacetic moiety and the involvement of the phenolic oxygen that loses its proton. The resulting extended electronic delocalization yields a colour change from orange to violet. With the increasing concentration of $\mathrm{Gd}(\mathrm{III})$, the violet colour becomes more pronounced. As such, free Gd(III) concentration is directly proportionate to the ratio of the violet colour (complexed dye) and the orange colour (uncomplexed dye). That is, the increase of Gd(III) causes an increase of the band at $563 \mathrm{~nm}$ in UV-Vis, while the band at $433 \mathrm{~nm}$ decreases $\left(\mathrm{Gd}^{\mathrm{free}} \propto \mathrm{Abs}^{573} / \mathrm{Abs}^{433}\right)$. This relationship is used in the construction of 
a standard curve, which in-turn allows for calculation of the content of unknown Gd(III) solution. $^{114}$

\subsubsection{Transmission electron microscopy and energy-dispersive X-ray spectroscopy}

Transmission electron microscopy (TEM) is a subset of microscopy which allows for characterization of materials with high spatial resolution. This is achieved through transmitting a beam of electrons through a sample with the final image constructed based on electrons that are received by the detector. The incident electrons are produced in the electron gun, where a cathode, typically a tungsten filament, is coupled with a high voltage source. This electronic heating provides valence electrons a sufficient amount of energy to emit into a vacuum. As the filament and the surrounding gun elements are kept at a high negative potential, the incident electrons are accelerated away from the cathode towards the anode at a great speed. The lens system is considered to be the most important aspect of the microscope, as it plays a crucial role in concentrating and focusing the radiant energy. The first lens the electrons pass through is used to produce a "point source" of illumination, effectively concentrating the electrons into a fine focused beam. The focused beam then passes through the second condenser lens, which can be varied in strength and serves to either converge the beam onto a small area of the specimen, spread it into a set of parallel rays incident on the specimen, or form into a divergent cone. An aperture is placed after this set of lenses and is used to eliminate off-axis rays and control the quality of beam convergence arriving at the specimen. ${ }^{115,116}$

Once the beam arrives at the sample, different densities of the materials will have a varying effect on the electrons; that is, electrons may be absorbed, scattered, or pass through. A series of lenses is used to magnify the sample image and project it onto a 
phosphorescent screen to convert the electron energy to visible light. These lenses include diffraction, intermediate, and projector lenses. One of the important aspects of TEM sample preparation is that specimens must be deposited on a mesh grids to allow for electrons to pass through. Upon image acquisition, characteristics such as particle size, grain size, lattice type (related back to the material being investigated), morphological information, crystallographic details, chemical composition, phase-type, and distribution can be observed. ${ }^{115-117}$

To further characterize a specimen, elemental composition can be assessed with energy-dispersive X-ray spectroscopy (EDS). Conveniently, TEMs are fitted with EDS capabilities, utilizing the already existing electron beam in analysis of near-surface elements of the sample. Specifically, the surface of the specimen is bombarded with the electron beam, monitoring emitted X-rays. In order for an X-ray photon to be released, first, the energy of the electron beam must be such that secondary electrons from the inner shell of the atom can be knocked out by inelastic collisions. Since this energetic state is unstable, relaxation occurs, whereby a weaker bound electron will move from the outer shell to fill the vacancy. This is accompanied by the emission of the excess energy, which is equivalent to the difference of the binding energies of the atomic shells. These X-rays can be related back to the identity of the elements being scanned, as each element possesses a specific wavelength that would be emitted. EDS can also be used for qualitative elemental analysis, providing information of variations of relative concentration of an element. ${ }^{118,119}$

\subsection{Fibrin clot production}

Not only is fibrin the basis of blood clots within the biological system, it has also been used as a hemostatic agent, adhesive, and sealant for tissue defects. The three 
categories pertain to inducing blood clotting in the presence of blood, connecting structures together through a fibrous network, and creating a barrier (sealing) to prevent leakage of gas or liquid, respectively. All of this can be achieved by combining two components: fibrinogen and thrombin, in the presence of calcium. This mimics the clotting cascade, during which activated thrombin converts fibrinogen into insoluble fibrin. Fibrin glue (or sealant) is biologically compatible, as it is biologically derived, and is therefore commonly used during surgeries. It has also shown utility in laboratory testing, involving studies like tissue engineering. All of the proof-of-concept work in this research was done using laboratory synthesized fibrin, employing methodology similar to that utilized during fibrin sealant production. ${ }^{120,121}$

\subsection{Magnetic resonance imaging theory}

\subsubsection{Nuclear spin and nuclear magnetic resonance}

MRI, a non-invasive and non-ionizing imaging modality, is governed by the same principles as nuclear magnetic resonance (NMR) which allows attainment of threedimensional images of deep and soft tissues with high resolution. Both techniques utilize the intrinsic property of non-zero nuclear spin. This is a property of nuclei with unpaired protons and neutrons, comprising of those with an odd atomic number and/or an odd atomic mass, thereby making them susceptible to an external the magnetic field. In biological organisms, hydrogen atoms (or protons) exist in abundance and fall into that category; therefore, most MRI scans essentially map the location of water $\left(\mathrm{H}_{2} \mathrm{O}\right)$ and fat (lipids). ${ }^{55,122}$ Protons, ${ }^{1} \mathrm{H}$, have a nuclear spin (I) of $1 / 2$ and possess both angular momentum from their rotating mass and magnetic moment form their charge. ${ }^{123-125}$ The intrinsic spin of the proton can be arbitrarily conceived as a spherical particle spinning about its own axis. 
Earth's surface magnetic field is not strong enough to influence the total alignment of these protons, as it is at least 10,000-times weaker than the current diagnostic scanners. However, when placed in a strong external magnetic field, denoted $\mathrm{B}_{0}$, a small majority of them will align in the same direction as the external magnetic field, acquiring the lower energy state and creating a net (or bulk) magnetization in the direction of $\mathrm{B}_{0}$, while the rest will align against the applied field (Figure 2.2). The number of possible spin states in an external magnetic field is represented using $\mathbf{2 I}+\mathbf{1}$, where, as above, $I$ is the nuclear spin. ${ }^{55,122,124}$ 126
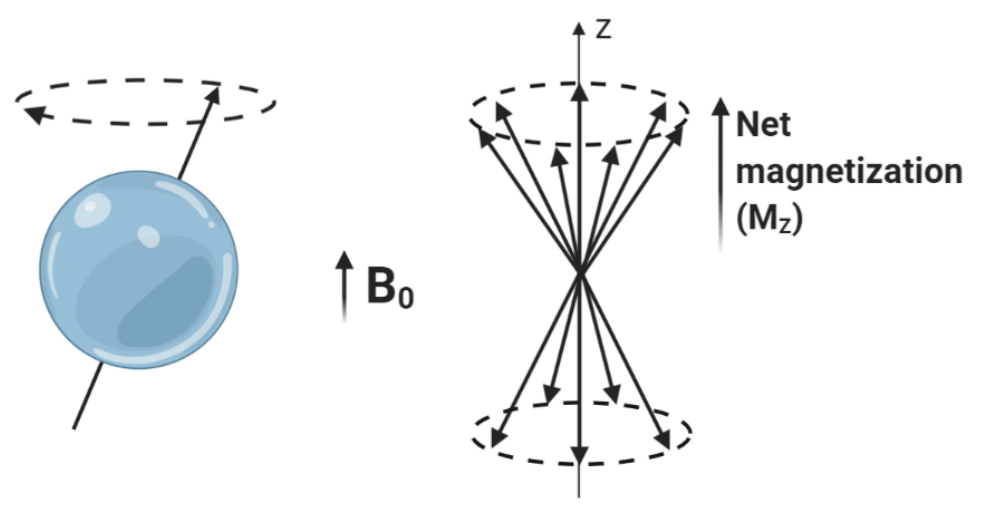

Figure 2.2: Schematic representation of the magnetic moment of a proton and its precession about $B_{0}$. Image produced in BioRender. ${ }^{55,127,128}$

The frequency of the proton's precession about the axis of the external magnetic field can be defined by, and is directly proportional to, the magnetic field strength, and is denoted as Larmor frequency $\left(\omega_{0}\right) .^{55,122,124,127}$ This is described by the Larmor equation 2.4

$$
\omega_{0}=\gamma B_{0} \quad 2.4
$$

where $\omega_{0}$ is the angular frequency of the protons in megahertz $(\mathrm{MHz}), \gamma$ is the gyromagnetic ratio, which is a proportionality constant of the unique nucleus, and $\mathrm{B}_{0}$ is the field strength in tesla $(\mathrm{T}) .{ }^{122,123}$ When considering the Larmor frequency in MRI, to which signal 
amplifiers are tuned, proton's gyromagnetic ratio of $42.58 \mathrm{MHz} / \mathrm{T}$ is used. Importantly, the only time a signal is detected is when the rotating component of net magnetization is in the $x y$-plane; therefore, at the ground state, when net magnetization is along the direction of the magnetic field $\left(\mathrm{M}_{\mathrm{Z}}\right)$, no signal is emitted. ${ }^{124,127}$

\subsubsection{Relaxation and its dependence on physical environment}

In an MR scan, a second alternating magnetic field, $\mathrm{B}_{1}$, is applied in order to tip the net magnetization of protons away from $\mathrm{B}_{0}$, resulting in magnetization into the transverse plane $(x y)$. Critically, $\mathrm{B}_{1}$ must be perpendicular to $\mathrm{B}_{0}$ and must correspond to resonance frequency of proton spins, which is in the radiofrequency (RF) range. Once the RF pulse is turned off, the time that it takes for these protons to relax back into their equilibrium alignment within $\mathrm{B}_{0}$ is referred to as relaxation time. In other words, relaxation is the process which drives the bulk magnetization to equilibrium. This time is dependent on the physical environment in which these protons exist, with two types, including longitudinal (T1 or spin-lattice) or transverse (T2 or spin-spin) relaxations. As such, in the same external field, different tissues will experience different relaxation times, producing different signals within a scan. ${ }^{55,123-125}$

Simply said, T1 relaxation refers to the process during which the energy acquired by the system from the RF pulse is transferred back into the surroundings (the "lattice"). This spin-lattice relaxation results in the recovery of net magnetization in the $z$-direction. The T1 time constant is typically recorded within seconds, during which $63 \%$ (or $1-1 / \mathrm{e}$, equation 2.5) of the equilibrium longitudinal magnetization is recovered. It is important to note that $\mathrm{T} 1$ only encompasses time-varying changes that occur at Larmor frequency and only arises from components of magnetic field fluctuations in the transverse 
plane. ${ }^{55,122,124,126}$ Mathematically, recovery of longitudinal magnetization occurs in an exponential fashion, which can be represented by equation 2.5 ,

$$
M_{z}(t)=M_{z}\left[1-e^{-t / T 1}\right] \quad 2.5
$$

where longitudinal magnetization at a given time, $t$, is denoted by $M_{z}(t)$ as it is along the $z$-plane. ${ }^{128,129}$

On the other hand, T2 relaxation is the result of the loss of phase coherence (or order) among the protons in the $x y$-direction. In this case, the relaxation occurs based on energy being transferred through spin-spin interactions during spin precession and this relates back to protons having a mutual influence on one another based on their magnetic properties. As such, T2 arises only from the energy transfer that occurs through magnetic interactions. Thus, T2 relaxation is induced by low frequency field variations and is mediated by fluctuations in all three, $x y z-$, directions. Notably, T2 is recorded within milliseconds and is the time that is required for transverse magnetization to decrease by $37 \% .^{55,122-124,126}$ In a similar fashion to $\mathrm{T} 1$ relaxation, recovery of transverse magnetization occurs in an exponential fashion and is mathematically represented by equation 2.6

$$
M_{x y}(t)=M_{z}\left[e^{-t / T 2}\right]
$$

where transverse magnetization at a given time, $t$, is denoted by $M_{x y}(t)$ as it is along the $x y$ plane, and $M_{x y}(0)=M_{z}$, as with application of a $90^{\circ}$ RF pulse, the initial longitudinal magnetization is completely converted to transverse magnetization. ${ }^{128,129}$ The summary of both $\mathrm{T} 1$ and $\mathrm{T} 2$ relaxation processes is provided in Figure 2.3. ${ }^{55}$ 


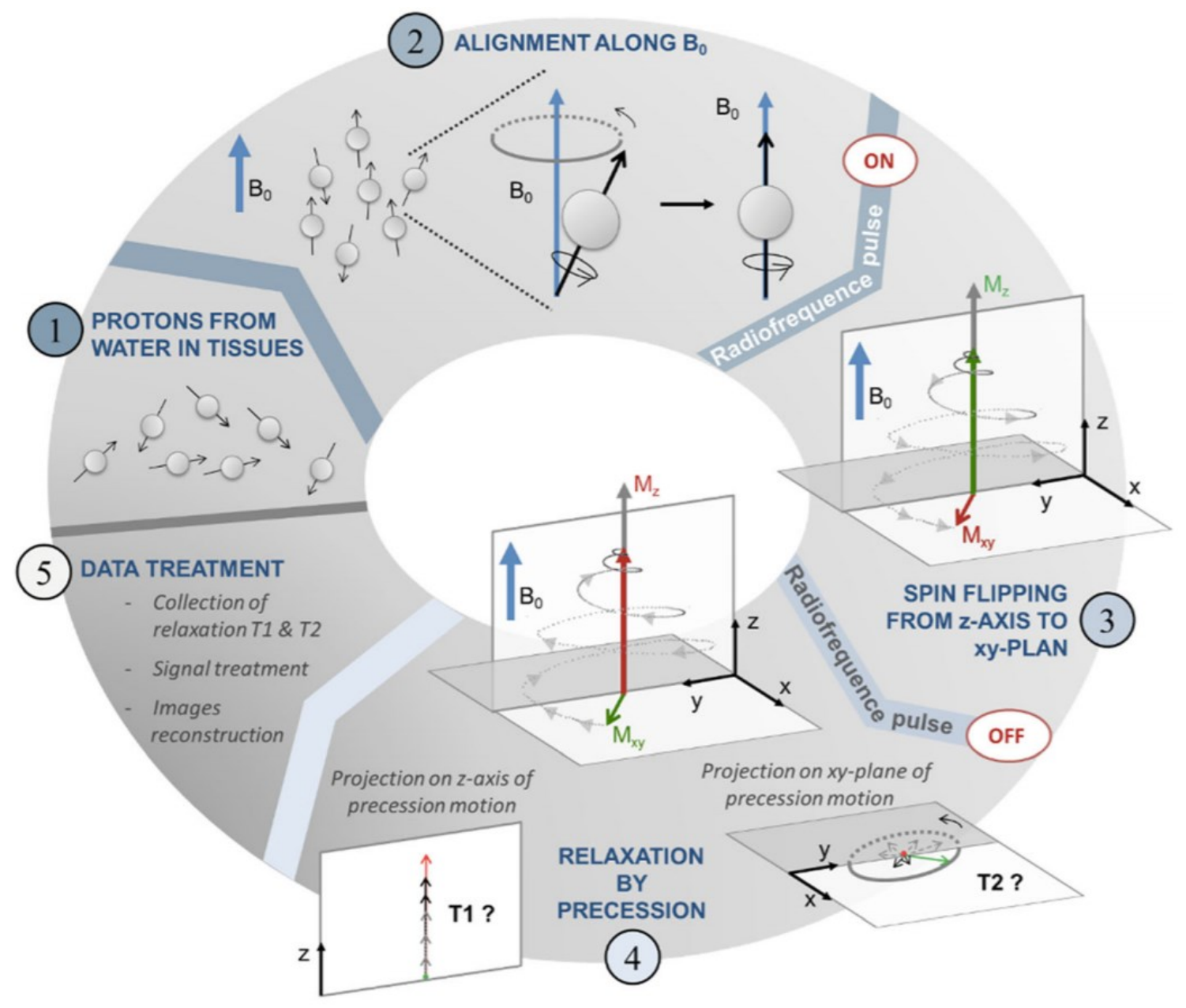

Figure 2.3: Schematic representation of nuclear spin of hydrogen from water molecules and its utilization in MRI, which is reconstructed in final image processing. Reprinted with permission from Springer Nature. ${ }^{55}$

Relaxation times are highly dependent on the physical environment in which protons exist and while T1- and T2-relaxation occurs nearly simultaneously, the two are independent of each other. Therefore, in non-contrasted MRI, tissues with short T1 values will appear brighter, while those with long T1 values will appear darker. Contrary to this trend, tissues with short T2 values will appear darker, while long T2 values will be viewed brighter. $^{123}$ 


\subsubsection{Repetition and echo times}

During a conventional MRI scan, in order to obtain optimally resolved images, two key parameters need to be considered. These parameters are repetition time (TR) and echo time (TE). TR is defined as the time between the start of each RF pulse and is usually measured in milliseconds. TE is the time between the initial application of an excitation RF pulse and the peak of a detected echo and is also measured in milliseconds. These parameters allow for fine-tuning of the observed differences in contrast of various tissues. To understand the role of each, a closer look at the effect of varying TR and TE is required. ${ }^{123,128,130}$

TR is crucial for T1 weighting, as it encompasses the relaxation period in the $z$ plane between two excitation pulses. Repeated excitation at fixed time interval is used in order to acquire the strongest signal associated with reoccurring exchange of excited nuclei and their surroundings. Given a TR that is long enough, all protons will have an opportunity to reach equilibrium alignment in the $z$-plane before the next excitation RF pulse is applied, thereby providing a bulk contribution to the regrowth of longitudinal magnetization. With that, the signal that is emitted by any given tissue will be relatively independent of $\mathrm{T} 1$ effects. However, if the time between the successive excitations is short, the amount of longitudinal relaxation present at the time of each sequential excitation will depend on the tissue's T1. This means that tissues that experience a short T1 will have a sufficient amount of time for relaxation to occur, thereby giving a large signal. Conversely, tissues with a long T1 will undergo partial relaxation, providing less longitudinal magnetization during the next pulse and giving a weaker signal. Therefore, the image that is acquired with a short TR has more T1 information and is T1-weighted. ${ }^{123,128,130}$ 
The other factor that determines tissue contrast is TE, which is related to T2 weighting. TE is the interval between the induction of the excitation RF pulse and collection of the MR signal. Immediately after a $90^{\circ}$ pulse is applied, the net transverse magnetization (in the $x y$-plane) is at its maximum value. The decay of this magnetization is dictated by T2. Therefore, choosing an echo time defines the influence of T2 on the final contrast of the image. The greater the $\mathrm{T} 2$ value, the longer transverse magnetization will persist, which means that, in order to match this and collect a strong T2 signal, a relatively long TE must be chosen. With this, tissues with a short T2 will have lost a significant amount of transverse magnetization and their signal will no longer be recorded. As such, images that are collected with a long TE are T2-weighted. In the interest of eliminating a T2 signal, a short TE may be chosen. At the time of collection, most tissues will not undergo a considerable amount of relaxation, which means their contribution will be small producing a weak signal. ${ }^{123,128,130}$

"Inversion recovery" sequences are commonly used for quantitative T1 assessment and T1-weighted imaging (and to some degree T2 assessment and imaging). They are similar to a conventional spin echo sequence, with an important difference of an additional $180^{\circ} \mathrm{C}$ pulse (inversion pulse) which precedes the usual $90^{\circ}$ excitation pulse. The inversion pulse of a predetermined power and duration is specifically geared towards inverting the longitudinal magnetization against $\mathrm{B}_{0}$, into the negative $z$-direction. Longitudinal magnetization is allowed to recover for a specific time interval, referred to as inversion time. The subsequently applied $90^{\circ}$ pulse refocuses the magnetization and is the same as in a spin-echo sequence. Overall, the "inversion recovery" sequence is as follows: $180^{\circ}$ time of inversion $-90^{\circ}$ acquire. The degree of recovery is dictated by the tissue's or the 
sample's T1 and the time between the two purses is denoted TI (time of inversion) or $\tau$ (tau). With short $\mathrm{T} 1$, full recovery of longitudinal magnetization may be achieved, while long T1 will only allow for partial recovery. Since the inversion pulse does not result in magnetization in the $x y$-plane, no signal is recorded in association with the pulse. It is after the $90^{\circ}$ excitation pulse is applied that the magnetization is tipped into the $x y$-direction. Relaxation towards equilibrium alignment with $\mathrm{B}_{0}$ is recorded at this point, with the acquired signal based on the precession within the $x y$-plane. In order to determine T1, the observed magnetization is expressed as a function of relaxation delay time $(\tau)$, varying tau through repeated scans. ${ }^{128,130,131}$ This is represented by equation 2.7 , which is integrated into MRI and NMR software,

$$
M(\tau)=M_{0}\left[1-2 \exp \left(-\frac{\tau}{T 1}\right)\right] \quad 2.7
$$

where $M(\tau)$ is the observed magnetization, $M_{0}$ is magnetization at equilibrium, acquired at various known values of tau, allowing to solve for T1. ${ }^{128-132}$

\subsubsection{MRI contrast materials}

\subsubsection{Paramagnetic species - gadolinium(III)}

When considering a collection of water molecules, consisting of two hydrogen atoms each, that is placed in a magnetic field, their rotation and translation in space must be acknowledged. When the water molecules rotate, they themselves induce an oscillating magnetic field due to the magnetic moment associated with the hydrogen atoms. If this rotation occurs at the resonance frequency $\omega_{0}$ (Larmor frequency), it will have an effect on the nearby protons, inducing transitions between the alignment states. This is referred to as proton-proton (or dipole-dipole) interaction and is precisely the mechanism of $\mathrm{T} 1$ 
relaxation. As such, it would be reasonable to assume that effects similar to this will adjust T1 relaxation. The extent of this interaction is, however, dependent on the distance between the interacting dipoles. Specifically, the strength is inversely proportional to the sixth power of the distance. For example, at a distance that is equivalent to the length of a typical chemical bond, (1 Ångstrom), the local magnetic effect will be considerable. ${ }^{128,130}$

Paramagnetic species are those with a large number of unpaired valence electrons, which makes them useful as MRI contrast agents. One such example is gadolinium ions (Gd(III)), which contain seven unpaired electrons the $4 \mathrm{f}$ orbitals, with nine coordination sites which can be used for chemical interactions. ${ }^{128,133,134}$ Unfortunately, "free" or unchelated Gd(III) is highly toxic in most biological systems. This toxicity is directly related to its ionic radius, which is nearly identical to that of calcium ions. As such, proteins are unable to distinguish between $\mathrm{Gd}(\mathrm{III})$ and calcium, which leads to $\mathrm{Gd}(\mathrm{III})$ binding calcium ion channels and other calcium-requiring proteins. Since calcium ions are involved in many processes, including cellular respiration, muscle contractility, and blood clotting, the presence of Gd(III) would have an antagonistic effect on these processes. ${ }^{123,134}$ Since gadolinium is highly toxic if administered as a free ion, its chelation has been well researched, with a number of bifunctional paramagnetic chelators available. ${ }^{128,131,133}$ Common examples include diethylenetriaminepentaacetic acid (DTPA) (Magnevist), 1,4,7,10-tetraazacyclododecane-1,4,7,10-tetraacetic acid (DOTA) (Dotarem), 1,4,7triazacyclononane-1,4,7-triacetic acid (NOTA) and others (Figure 2.4). ${ }^{135}$ The main effect of gadolinium chelation is the decrease of the number of water molecules that can interact with the ion, due to the decrease in available coordination sites. Nevertheless, a Gd(III) 
complex is able to interact with nearby protons, which alters their relaxation properties. $^{128,131,133}$
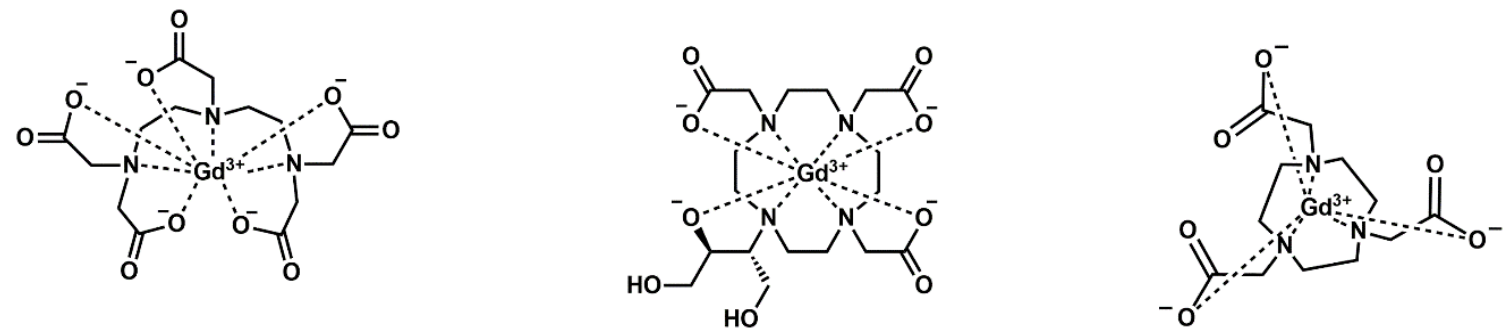

Figure 2.4: Paramagnetic chelators diethylenetriaminepentaacetic acid (DTPA) (Magnevist), 1,4,7,10tetraazacyclododecane-1,4,7,10-tetraacetic acid (DOTA) (Dotarem), 1,4,7-triazacyclononane-1,4,7triacetic acid (NOTA). ${ }^{135}$ Structures drawn in ChemDraw.

The ground electric state of Gd(III) has a nuclear spin of 7/2, which corresponds to a very large magnetic moment, allowing for these species to act as a strong dipole. ${ }^{128,136} \mathrm{In}$ the same manner as hydrogens have an effect on one another through dipole-dipole interactions, Gd(III)-based agents will have an effect on neighbouring hydrogens. Therefore, paramagnetic relaxation of water protons will be the result of interactions between the nuclear spin and the fluctuating local magnetic field as a function of the unpaired electrons of Gd(III)-based agents. Importantly, this effect only occurs when the distance between the nearby nuclei and the metal ion is short enough for interaction with the inner coordination site to occur. Nevertheless, the dipole-dipole effect of this interaction is significant and results in a measurable effect on longitudinal relaxation rate, denoted by $\mathrm{R} 1=1 / \mathrm{T} 1$, of water protons. Another important factor which affects relaxation rates is the concentrations of the paramagnetic substance. This is represented by equation 2.8 , often used to determine the effectiveness of a contrast agent (CA) 


$$
\frac{1}{T_{i, o b s}}=\frac{1}{T_{i, d}}+r_{i}[C A], \text { where } i=1,2
$$

with $T_{i, \text { obs }}$ is the observed relaxation time (or observed T1/T2) and $T_{i, d}$ is the relaxation time in the absence of the paramagnetic substance. When the observed relaxation is plotted as a function of the concentration of Gd(III)-based agent, the slope of the equation defines $r_{i}$, which is relaxivity of the given contrast agent. ${ }^{55,137-139}$

\subsubsection{Superparamagnetic substances - iron oxide nanoparticles}

Superparamagnetic substances are an attractive alternative to the commonly used paramagnetic Gd(III)-based agents. One example of such substance is superparamagnetic iron oxide nanoparticles (SPIONs). Superparamagnetic properties of $\mathrm{Fe}_{3} \mathrm{O}_{4}$ arise from the presence of two different iron atoms, $\mathrm{Fe}^{2+}$ and $\mathrm{Fe}^{3+}$, with 4 and 5 unpaired valence electrons, respectively, in a rigid crystal lattice. Their opposing moments are unequal, which results in a net spontaneous magnetic moment and is manifested as highly susceptible paramagnet in the presence of a magnetic field. Due to local field inhomogeneities, which are thereby produced based on these magnetic moments, proton dephasing is greatly accelerated, shortening their T2 relaxation. This magnetic susceptibility leads to a pronounced loss of signal in T2-weighted imaging. ${ }^{70,123,140}$

Superparamagnetic relaxation mechanism is built upon the aforementioned paramagnetic systems. The main contributor to the accelerated relaxation of the nearby protons is the energy exchange of the said protons with the electrons in the first hydration sphere of the paramagnetic ion, which are highly mobile within the crystal lattice. The efficiency of SPION-based contrast agents can be assessed using equation 2.8 , as described above. As such, the contrast capabilities of SPIONs are also related to the concentration of the material present within the system. ${ }^{70,123,139}$ 


\subsection{Computed tomography}

Computed tomography (CT) is a well-established technique that uses the same physical principles as a conventional radiography. Namely, both use ionizing energy, Xray, that pass through an object. X-ray is a type of penetrating electromagnetic radiation, and the degree of the said penetration is based on the energy of the incident X-ray, as well as the material which it passes through. Specifically, with an X-ray source and a detector positioned on opposite sides of one another and the sample in the middle, cross-sectional (or tomographic) images are produced through mathematical reconstruction based on the X-ray intensities received by the detector. The images are then combined to generate a final three-dimensional rendition. When X-rays are generated, high voltage is used to accelerate electrons from the cathode towards an anode. These electrons release electromagnetic radiation which is dictated by the energy of the incident electron. Most CT scanners work with voltages in the range of $40 \mathrm{kVp}$ to $150 \mathrm{kVp} .55,141,142$

The mass attenuation is dictated by the physical properties and density of the tissues along the path of the X-ray. That is, X-ray attenuation follows the Beer-Lambert exponential attenuation law and is based on density $(\rho)$ and the anomic number $(Z)$ of the specimen: the higher the density or the atomic number, the higher the X-ray attenuation. For example, dense tissues, like bones or calcification, experience high attenuation and penetrable tissues experience variable degrees of low attenuation. It is important to note the same parameters are taken into account when materials are picked to be effective contrast agents. ${ }^{55,143,144}$ This relationship is demonstrated by the formula used to calculate X-ray absorption coefficient $(\mu)$ :

$$
\mu \approx \frac{\rho Z^{4}}{A E^{3}}
$$


where $A$ is the atomic mass of the element and $E$ is the $\mathrm{X}$-ray energy used. A scanned specimen may be divided into a matrix of rectangular boxes, otherwise known as voxels. Within each voxel, there will be a certain amount of X-ray attenuation which is related back to the type of tissues that the ray is passing through, and these values are used to produce the final image of the scanned object.

The ability of matter to attenuate X-rays is defined by Hounsfield Units (HU). Material's HU value can be calculated by:

$$
H U=\frac{\left(\mu-\mu_{\text {water }}\right)}{\left(\mu_{\text {water }}\right)} \times 1000
$$

where $\mu_{w a t e r}$ is the X-ray attenuation coefficient of water. All attenuation is normalized to that of water, which is assigned the baseline density value of $0 \mathrm{HU}$. Lungs (air) and bone are two outliers which have opposing density values of $-1000 \mathrm{HU}$ and $1000 \mathrm{HU}$, respectively. Unfortunately, permeable soft tissues, which dominate biological systems, do not exhibit such attenuation, and typically produce density values in the range of 30 to 100 HU. As a result, unenhanced soft-tissue imaging is rarely highly resolved, proving it challenging to identify the interface between adjacent entities. Consequently, a number of $\mathrm{CT}$ contrast materials have been developed in order to increase sensitivity and resolution, provide opportunities for deduction of biochemical information, and enable evaluation of biological function or performance. ${ }^{143-145}$

\subsubsection{Computed tomography and fluoroscopy contrast agents}

Some of the most common agents include iohexol (Omnipaque, GE Healthcare), iopromide (Ultravist, Bayer Healthcare), iodixanol (Visipaque, GE Healthcare), and iopamidol (Isovue, Bracco Imaging Canada) (Figure 2.5). ${ }^{143}$ 


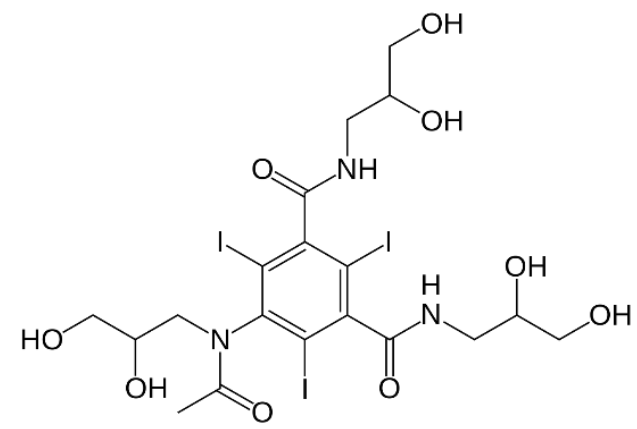

Figure 2.5: Structure of a commonly used CT contrast material, iohexol, or Omnipaque (5-[N-(2,3Dihydroxypropyl)acetamido]-2,4,6-triiodo-N,N'-bis(2,3-dihydroxypropyl)isophthalamide). ${ }^{143}$ Structure drawn with ChemDraw.

The working principle behind these molecules is based on the presence of three iodine ions at the 2,4,6-positions of the benzene ring. Iodine, with a high atomic number, $\mathrm{Z}=53$, possesses three important characteristics: it is highly dense and shows excellent $\mathrm{X}$ ray attenuation, it is able to firmly bind to the benzene ring, and it can be relatively low in its toxicity. Most of the currently utilized CT contrast materials are non-ionic, which circumvents the possibility of interactions with biological structures, including peptides and cell membranes, and helps lower renal toxicity and injection pain as it pertains to high osmolarity of ionic species. Subsequent to the injection of the contrast material, an incident $\mathrm{X}$-ray of an equal or slightly greater energy than the binding energy of the K-shell electron of iodine is administered. This energy is defined as the absorption edge $(k)$ and is proportionate to the atomic number of the element. As such, the higher the atomic number, the higher the $k$ value. In the presence of X-ray attenuating contrast material, an increase in absorption coefficient is observed, thereby improving visualization of the target tissue. Specifically, at concentrations of $1 \mathrm{mg}$ of iodine $/ \mathrm{mL}$ an increase of approximately $26 \mathrm{HU}$ can be observed at $120 \mathrm{kVp} \cdot{ }^{143,146,147}$ 


\subsection{Image analysis - ImageJ software}

ImageJ is a widely used scientific image-analysis program with capabilities to open multitude of image file formats. With countless options for image processing and analysis, this software has been used for scientific assessment of images, including but not limited to those acquired in clinical research. ${ }^{148,149}$ In this research, ImageJ was used in analysis of fluorescence microscopy images, and both MRI and CT images. The "Measure" function allowed for quantification of average pixel intensities of fibrin and blood clot samples. These measurements were related back to the intensity of fluorescence of contrast, respectively. In both MRI and CT imaging, samples were scanned acquiring a number of slices throughout. When analyzing MRI and CT images, to facilitate consistent measurements, slices of the same depth were chosen for all samples. This ensured that there is no bias towards a specific area, which may show higher contrast than others. In addition, it allowed for an assumption that, if contrast enhancement is visible in association with the positive treatment, a sufficient amount of time has passed for the contrast material to interact with the target. Therefore, since the control treatments were designed in such a way that they were similar to the positive treatments, with the exception of the aptamer sequence, they too have been allowed enough time to interact with the sample. 
Chapter 3: Developing aptamer-conjugated gadolinium contrast media to target fibrin clots in magnetic resonance imaging 


\subsection{Statement of contributions}

A Koudrina performed chemical synthesis of contrast materials and prepared MRI samples. JA Zurakowski assisted with the phantom MRI samples as an undergraduate student. EM McConnell assisted with the initial stages of the project, as well as experimental design. MC DeRosa assisted with the experimental design. GO Cron performed all MRI scans at The Ottawa Hospital. EC Tsai is the clinical collaborator on this project.

\subsection{Resulting publications}

This chapter is based on an article entitled "Exploring unique contrast properties of aptamer-gadolinium conjugates in magnetic resonance imaging for targeted imaging of thrombi" submitted for publication in ACS Applied Materials \& Interfaces (Koudrina A,

McConnell EM, Jurakowski JA, Cron GO, Chen S, Tsai EC, DeRosa MC. ACS Applied Materials \& Interfaces - invited contribution. Manuscript ID: am-2020-166668). 


\subsection{Introduction}

Fibrin formation is the integral component of blood clotting, which is necessary to prevent hemorrhage but is also implicated in a wide range of disorders and disease states. In most cases, the formation of blood clots is the natural response of the biological system to a blood vessel injury and is necessary in cases of trauma or surgery. ${ }^{150,151}$ However, any malfunction within the clotting cascade may lead to numerous thrombotic complications, resulting in hypercoagulation and vascular occlusions. ${ }^{34,152,153}$ Stroke is an example of an acute vascular event in which fibrin can play a major role. The significance of fibrin is dictated by the type of stroke; that is, in the event of a hemorrhagic stroke, fibrin assists in sealing the site of a rupture to stop bleeding, which is favorable. While in ischemic stroke, clots that result in vascular occlusions lead to obstructed flow within a blood vessel (thrombosis) thereby making the presence of fibrin unfavourable. ${ }^{153}$ Magnetic resonance imaging (MRI) is one of the imaging modalities that can be used to investigate a variety of pathologies, including those described above. ${ }^{154}$

MRI is a non-invasive and non-ionizing technique which exploits tissue-dependent relaxation times of magnetically excited protons to produce three-dimensional images of soft tissues with high spatial resolution. This technology uses the same principle as nuclear magnetic resonance, utilizing the intrinsic property of nuclear spin. Specifically, hydrogen atoms can absorb and emit radio frequency energy while under the influence of an external magnetic field. In biological organisms, hydrogen atoms are abundant within different environments and in the same external field, tissues will experience different relaxation times, producing different signals within a scan. ${ }^{14,155}$ Magnetic resonance angiography may require contrast material injection into a vein, which affords the dye direct access to the 
bloodstream, in order to visualize blood vessels. ${ }^{44}$ One of the most common materials used in MRI is ionic gadolinium ( $\mathrm{Gd}(\mathrm{III}))$, a paramagnetic lanthanide that is able to enhance longitudinal (T1) and transverse (T2) relaxation rates by altering the relaxation time of nearby water protons. $\mathrm{Gd}(\mathrm{III})$ is highly toxic as a free ion; therefore, it is administered as part of a stable chelate with a multi-dentate ligand, such as diethylenetriaminepentaacetic acid (DTPA), 1,4,7,10-tetraazacyclododecane-1,4,7,10-tetraacetic acid (DOTA), 1,4,7triazacyclononane-1,4,7-triacetic acid (NOTA) and others. ${ }^{53,135,155,156} \mathrm{Gd}(\mathrm{III})$-based agents adjust longitudinal relaxation rates of protons predominantly through complexation in the inner metal coordination sphere, with paramagnetic influence subsequently propagated to the bulk water protons. Consequent shortening of T1 relaxation times yields increased image intensity (hyperintense) of T1-weighted images. Meanwhile, adjustment of transverse relaxation rates occurs when outer-sphere effects become more pronounced. That is, when the paramagnetic center is not accessible, bulk water protons will be influenced as they diffuse in the surroundings of the paramagnetic ion. Shortening of T2 relaxation times results in reduced signal intensity (hypointense) of T2-weighted images. $\mathrm{Gd}(\mathrm{III})$ contrast agents are typically used as positive agents, as their predominant effect is that of T1 shortening. Solomon-Bloembergen-Morgan theory states that parameters affecting this relaxivity is water exchange times, rates of tumbling of the CAs, and availability of coordination sites to the inner-sphere of CAs. It is important to consider that this theory mainly applies to CAs that are relatively dilute. ${ }^{137,157-159}$

Although these techniques are effective methods of diagnosis, they suffer from a number of limitations. Angiography techniques cannot determine the exact site of where the blood vessel ruptured unless there is active bleeding. This is, in part, due to blood clots 
being difficult to differentiate from adjacent tissue. ${ }^{154}$ Identification of where the hemorrhage occurred is important in determining optimal management. For example, in patients with multiple aneurisms, detection of which aneurysm bled would have an impact on which dilation requires priority management to prevent rebleeding. This is especially important because, typically, vascular occlusions are identified by looking for voids in contrast flow. This makes assessment of the blood vessel past the point of visualizable contrast difficult. As a result, clots that are present within those voids are not visible, and therefore, not managed. ${ }^{53,154,160}$ In addition to the lack of specificity, millimolar concentrations are required in order to acquire sufficient contrast enhancement. ${ }^{14,155,161}$ This gives rise to undesirable side-effects such as nephrogenic systemic fibrosis in patients with kidney problems, as well as tissue accumulation, including in the brain. ${ }^{156}$ In many cases, the outcomes of the vascular conditions described above would be optimized given the exact location of a blood clot, assisting in a more precise diagnosis and effective management. One way to overcome the lack of resolution between the clots and the rest of the blood is by optimizing the distribution of the contrast agent within the biological system. In this work, we investigated the feasibility of combining existing contrast materials with molecular recognition technology in order to produce targeted fibrin clot contrast agents.

Aptamers are single-stranded oligonucleotides that are able to assume higher order structures in order to bind their respective target with high affinity and selectivity. They are selected through an in vitro process of systematic evvolution of ligands by exponential enrichment (SELEX).$^{5-7}$ Since their discovery, aptamers have been used as molecular recognition agents and offer several advantages over more established affinity elements 
such as antibodies, including superior stability and the ability to be chemically synthesized and modified, while being non-immunogenic and non-toxic within the biological system. ${ }^{14}$ Aptamer-targeted materials, including contrast and therapy agents, have been investigated by many groups over the last several decades. In relation to the coagulation cascade, aptamers have been probed as alternatives to the currently existing anticoagulant therapies. As such, a number of candidates successfully modulating or interfering with coagulation have been the subject of intense study. ${ }^{150,151,162}$ For example, Sullenger group introduced a cocktail of aptamers able to target factor Xa and prothrombin, two proteins within the same cascade, and successfully arrest blood clot formation in a highly procoagulant setting. ${ }^{163}$ As fibrin is associated with a number of aforementioned pathologies, it is a particularly interesting target for selective delivery of contrast agents. Indeed, others have investigated fibrin-targeting contrast media, highlighting the importance of the development of a method which allows for precise detection and characterization of whole-body thrombus. ${ }^{48,164-166}$ The most recently reported fibrin targeting contrast agent was reported by the Caravan group consisting of three fibrin-specific peptides used in positron emission tomography (PET). ${ }^{167}$

An aptamer was previously selected to bind fibrinogen, which is involved in the cascade production of fibrin. ${ }^{28,168}$ More specifically, fibrin is formed through thrombincatalyzed polymerization of fibrinogen monomers. ${ }^{5-7}$ We hypothesized that the previously characterized fibrinogen aptamer (FA) would retain some binding affinity towards the polymerized form, fibrin, as long as enough of the structural features of the fibrinogen monomers would be available for each FA to bind to them. As such, the goal of this work was to confirm that FA would bind fibrin with high affinity and selectivity, as well as to 
develop and characterize Gd(III)-loaded conjugates to be subsequently used in binding experiments and imaging studies.

\subsection{Materials and methods}

\subsubsection{Chemicals and instruments}

Maleimido-monoamide-DOTA (1,4,7,10-Tetraazacyclododecane-1,4,7-tris-acetic acid-10-maleimidoethylacetamide) and maleimido-monoamide-NOTA $\quad(1,4,7-$ Triazacyclononane-1,4-bis-acetic acid-7-maleimidoethylacetamide) were purchased from Macrocyclics (Plano, TX, USA). Fibrin from human plasma, collagen, fibrinogen, dithiothreitol (DTT) and xylenol orange sodium salt were all obtained from Sigma-Aldrich. Thrombin solution was purchased from Haematologic Technologies Inc. (Essex Junction, VT, USA). Other buffering agents were purchased from BioShop (Burlington, ON, CAN).

Deionized water was prepared using Millipore (Billerica, MA, USA) Milli-Q water system and used to prepare all buffers.

Fibrinogen and FB139 aptamers were synthesized on a BioAutomation MerMade6 oligonucleotide synthesizer (Plano, TX, USA), using standard phosphoramidite chemistry. Phosphoramidites and modifiers, including thiol-modifier C6 S-S and 6-fluorescein phosphoramidite (6-FAM), activator, deblock, capping, and oxidizing reagents were obtained from Glen Research (Sterling, VA, USA). Ultra-High Purity 5.0 argon was purchased from Praxair Canada (Mississauga, ON, Canada). Standard support columns and acetonitrile were purchased from BioAutomation (Plano, TX, USA). All DNA was purified using Glen-Pak cartridges purchased from Glen Research (Sterling, VA, USA) using the appropriate protocol for the modifier used during the synthesis. Subsequent to DOTA/NOTA conjugation, desalting and separation of DNA-DOTA/NOTA conjugates 
from free Gd(III) after chelation was achieved using Amicon-Ultra $0.5 \mathrm{~mL} 3 \mathrm{kDa}$ cut-off ultra-centrifugal units purchased from Fisher Scientific Canada (Ottawa, ON, Canada). Aptamers, DNA-DOTA/NOTA conjugates and Gd(III)-DNA-DOTA/NOTA conjugates were all quantified by monitoring absorbance at $260 \mathrm{~nm}$ using a Varian (Palo Alto, CA, USA) Cary 300 Bio UV-visible spectrophotometer (Varian, Palo Alto, CA, USA). All purified DNA and its conjugates were sent to Novatia, LLC (Monmouth Jct, NJ, USA) in order to obtain molecular weight confirmation of the products by ESI-MS. Microscale thermophoresis (MST) analysis was performed by 2bind (Regensburg, Germany) using Monolith NT.115 Pico. Blood was obtained from The Ottawa Hospital Research Institute with approval from the Ottawa Health Science Network Research Ethics Board, OHSNREB (application \# 20150544-01H). All MR Imaging was done using an animal GE/Agilent MR901 Discovery 7T MRI Scanner (The University of Ottawa, Preclinical Imaging Core). Illustrations were prepared in BioRender.

Table 3.1: DNA aptamer sequences and their associated abbreviations

\begin{tabular}{|l|l|c|}
\hline $\begin{array}{l}\text { Aptamer } \\
\text { name }\end{array}$ & Sequence (5'-3') & Ref \\
\hline FA & $\begin{array}{l}\text { CCTTCGTTGTCTGCCTTCGTAGGACCGCAGACATCGACG } \\
\text { CAGGGAAATTCCGCAAGTCCAGCCAAATGCCACCCTTCA } \\
\text { GAATTCGCACCA }\end{array}$ & 168 \\
\hline FB139 & $\begin{array}{l}\text { ATACCAGCTTATTCAATTAATCGCATTACCTTATACCAGC } \\
\text { TTATTCAATTACGTCTGCACATACCAGCTTATTCAATTAG } \\
\text { ATAGTAAGTGCAATCT }\end{array}$ & 169 \\
\hline
\end{tabular}


Table 3.2: Aptamer abbreviations and detailed component description

\begin{tabular}{|c|c|}
\hline Abbreviation & Aptamer details \\
\hline FA & Fibrinogen-binding aptamer \\
\hline FB139 & $\begin{array}{l}\text { Fumonisin B1-binding aptamer used as negative control based on } \\
\text { similarities in length and characteristics }\end{array}$ \\
\hline Abbreviation & Component details \\
\hline 6-FAM FA & $\begin{array}{l}\text { Fluorescently modified (6-fluorescein phosphoramidite) fibrinogen } \\
\text { aptamer }\end{array}$ \\
\hline Thiol-FA & $\begin{array}{l}\text { Thiol C6 S-S modified fibrinogen aptamer used in conjugation } \\
\text { protocol }\end{array}$ \\
\hline $\begin{array}{l}\text { DOTA/NOTA- } \\
\text { FA }\end{array}$ & $\begin{array}{l}\text { Fibrinogen aptamer conjugated to DOTA or NOTA bifunctional } \\
\text { chelator }\end{array}$ \\
\hline $\begin{array}{l}\text { Gd- } \\
\text { DOTA/NOTA- } \\
\text { FA }\end{array}$ & $\begin{array}{l}\text { Gadolinium-loaded DOTA or NOTA chelator, functionalized with } \\
\text { fibrinogen aptamer }\end{array}$ \\
\hline
\end{tabular}

\subsubsection{Preparation of Gd-DOTA/NOTA-FA to be used as an MRI probe}

Thiol-FA (60 nmol) was vortexed in $75 \mu \mathrm{L}$ of $50 \mathrm{mM}$ Tris (pH 8.4) containing 100 $\mathrm{mM}$ dithiothreitol (DTT) at ambient temperature for 30 minutes in order to cleave the 5' disulfide in preparation for conjugation with the chelator, maleimido-monoamideDOTA/NOTA. Cleaved FA was washed using BioRad Micro Bio-Spin Chromatography columns, using EDTA buffer (7 mM, pH 7.0) and subsequently de-salted using Amicon Ultra 0.5 mL Centrifugal Filters with HEPES buffer (50 mM, $7 \mathrm{mM}$ EDTA, pH 7.0). The de-salted product was then combined with large excess $(1.27 \mu \mathrm{mol})$ of maleimidomonoamide-DOTA/NOTA in HEPES buffer and vortexed for 3 hours, at ambient temperature. Following chelation, FA-DOTA/NOTA conjugates were de-salted using Amicon Ultra $0.5 \mathrm{~mL}$ Centrifugal Filters with deionized water in order to remove unreacted maleimido-monoamide-DOTA/NOTA. 
FA-DOTA/NOTA conjugates were then resuspended in Tris buffer $(50 \mathrm{mM}, 140$ $\mathrm{mM} \mathrm{NaCl}, \mathrm{pH} 7.4)$ and combined with $\mathrm{Gd}(\mathrm{III})$ in ammonium acetate buffer $(0.5 \mathrm{M}, \mathrm{pH} 6)$ in 1:1 molar equivalent. This mixture was shaken overnight at ambient temperature and desalted using Amicon Ultra $0.5 \mathrm{~mL}$ Centrifugal Filters with deionized water. Thiol-FA and -FB139 and its conjugates were sent to Novatia, LLC (Monmouth Jct, NJ, USA) in order to obtain molecular weight confirmation of the products by ESI-MS. To determine the concentration of loaded Gd(III), the wash-through was tested for free $\mathrm{Gd}(\mathrm{III})$, using xylenol orange test. A calibration curve was constructed using a range of concentrations of Gd(III) complexed with a constant amount of xylenol orange. ${ }^{114}$ The same amount of dye was added to the wash-through from the loaded FA-DOTA/NOTA. The absorbances of the standard curve solutions and the excess salt fraction were measured and the amount of free Gd(III) was determined against the standard curve constructed.

\subsubsection{Assessment of binding}

\subsubsection{Fluorescence microscopy}

Binding of fibrinogen aptamer to fibrin polymer was initially assessed using fluorescence. Fibrin clots were synthesized by combining a solution of $20 \mathrm{mg} / \mathrm{mL}$ of fibrinogen, a solution of $0.1 \mathrm{M} \mathrm{CaCl}_{2}$ and $240 \mathrm{IU} / \mathrm{mL}$ of thrombin in phosphate buffered saline (PBS) , pH 7.4. ${ }^{170,171}$ The mixture was incubated for an hour at $37{ }^{\circ} \mathrm{C}$ and the resulting clot was cryosectioned on a Leica CM1900 cryostat to 60 microns. Fibrin slices were subsequently fixed onto a glass slide. In the binding study, $20 \mu \mathrm{L}$ of $5 \mu \mathrm{M}$ 6-FAM FA or 6-FAM FB139 were blotted over the clot slices and incubated for 30 minutes at room temperature. Slices used in the binding kinetics assessment were prepared in the same manner and incubated for 30 seconds, 1, 5, 10 and 30 minutes (see additional materials and 
methods in supporting info). All samples were produced in triplicate. Unbound excess DNA was washed away with PBS, pH 7.4 and the clots were imaged with a BioRad FluoroMicro fluorescence microscope under blue and green channels, with excitation wavelengths of $355 \mathrm{~nm}$ and $480 \mathrm{~nm}$, respectively. Fluorescence was assessed with ImageJ, measuring average pixel intensity.

\subsubsection{Microscale thermophoresis}

Cy5-FA was kept at a constant concentration $(20 \mathrm{nM})$ and was tested against fibrinogen and fibrin targets, in order to compare binding constants of the monomeric and polymeric forms. Fibrinogen was titrated from $2 \mu \mathrm{M}$ (in binding buffer, $20 \mathrm{mM}$ Tris $\mathrm{HCl}$ $\mathrm{pH}$ 7.6, $300 \mathrm{mM} \mathrm{NaCl}, 5 \mathrm{mM} \mathrm{MgCl} 2$ ) down in 16 1:1 dilution steps, with the concentration range of $2 \mu \mathrm{M}$ to $61 \mathrm{pM}$. The samples were analyzed at $25^{\circ} \mathrm{C}, 3 \% \mathrm{LED}$ power, and $40 \%$ laser power (1475 $\mathrm{nm} \pm 15 \mathrm{~nm}$, power: $120 \mathrm{~mW} \max )$. Similarly, fibrin was prepared to $1.94 \mu \mathrm{M}$ in binding buffer in the presence of $19.4 \mathrm{mM} \mathrm{NaOH}$ (for fibrin solubilization), with the solution neutralized with $15 \mathrm{mM} \mathrm{HCl}$ to avoid precipitation. The final concentration range was $1.94 \mu \mathrm{M}$ to $59.2 \mathrm{pM}$, and was analyzed at $25^{\circ} \mathrm{C}, 3 \% \mathrm{LED}$ power, and $40 \%$ laser power $(1475 \mathrm{~nm} \pm 15 \mathrm{~nm}$, power: $120 \mathrm{~mW} \max )$. Dissociation constants were determined for each target, based on fluorescence in unbound and bound states.

\subsubsection{Assessment of relaxivity}

\subsubsection{NMR assessment (9.4 T)}

A $400 \mathrm{MHz}$ (9.4 T) Bruker (Peabody, Massachusetts) NMR Spectrometer (Carleton University) was used for determination of T1 relaxation. Gadovist (clinical Gd-DO3A formulation), Gd(III)-NOTA-FA, and Gd(III)-NOTA-FA + fibrin were dried down and 
subsequently reconstituted in $\mathrm{D}_{2} \mathrm{O}(99.9 \%)$ to concentrations of $0.003,0.006,0.012,0.025$, and $0.050 \mathrm{mM}$ (based on $\mathrm{Gd}(\mathrm{III})$ concentration). Note, the incubations of Gd(III)-NOTAFA + fibrin were prepared in a 1000:1 ratio. Due to limited availability of the sample, coaxial insert $-4 \mathrm{~mm}$ tube with a $50 \mathrm{~mm}$ was used in conjunction with the standard $5 \mathrm{~mm}$ NMR tube. The insert tubes were silanized using Sigmacote reagent as per the manufacturer's instructions. Briefly, a $4 \mathrm{~mm}$ long needle was used to inject the reagent, removing it a few minutes later. The tubes were dried overnight in the fume hood and the reagent was subsequently fixed in the oven at $100^{\circ} \mathrm{C}$ for 30 mins. They were then rinsed with deionized water and dried again at $100^{\circ} \mathrm{C}$. An inversion recovery calculation was done automatically using Jeol Delta NMR Software. Molar relaxivity was obtained through the slope of the linear fit equation for relaxation rate $(1 / \mathrm{T} 1)$ vs concentration.

\subsubsection{Clinical MRI assessment (7 T)}

Gadovist, Gd(III)-NOTA-FA, and Gd(III)-NOTA-FA + fibrin samples were prepared to the same concentrations as above, in PBS buffer, $\mathrm{pH}$ 7.4. Both $\mathrm{T} 1$ and $\mathrm{T} 2$ scans were performed to compare the ability of Gd(III)-NOTA-FA to produce contrast enhancement and assess the effect of target binding. To allow for easy assessment, the fibrin target was solubilized in $1 \mathrm{M} \mathrm{NaOH}$ by dissolving $50 \mathrm{mg}$ of lyophilized fibrin powder in $2 \mathrm{~mL}(25 \mathrm{mg} / \mathrm{mL})$. The final concentration was determined with UV-Vis, monitoring the peak at $280 \mathrm{~nm}$. Gd(III)-NOTA-FA was added to fibrin at 1000:1 ratio.

The pulse sequence for T1 map followed inversion recovery technique, with 43 different inversion times from 130 to $15000 \mathrm{~ms}$; repetition time $4 \mathrm{~ms}$; echo time $1.8 \mathrm{~ms}$; matrix size $256 \times 256$. The pulse sequence for $\mathrm{T} 2$ map followed the spin echo technique, with 8 time points at 11.8, 17.6, 23.5, 29.4,35.3, 41.2, 47.0, $52.9 \mathrm{~ms}$; repetition time 6000 
ms; matrix size $256 \times 256$. Volume resonator body $150 \mathrm{~mm}$ coil was used for all relaxivity measurements.

The signal intensities were measured using a predetermined round region of interest (ROI) with ImageJ software. Relaxation rate $\left(1 / T_{n}, n=1,2\right)$ was plotted against concentration, and the slope of the linear fit was used to deduce relaxivity.

\subsubsection{MRI scans}

\subsubsection{Relaxivity measurements in the presence of non-solution-based target}

All samples were embedded in $0.6 \%$ agarose gel and prepared in triplicate. Powdered fibrin from human plasma (Sigma-Aldrich) was used to assess the effects of binding a non-solution-based target on relaxation rates. Collagen (Sigma-Aldrich) was used as the protein control, and FB139 aptamer was used as the aptamer control. All aptamer conjugates, Gd(III)-DOTA-FA, Gd(III)-NOTA-FA, and Gd(III)-DOTA-FB139, were suspended in PBS buffer, $\mathrm{pH} 7.4$, to concentration of $50 \mu \mathrm{M}$ and $150 \mu \mathrm{L}$ were applied directly on top of agarose gel. The aptamer was allowed to diffuse into the gel and interact with the target for 48-hours (refer to supporting information). The pulse sequence for T2 map followed the spin echo technique, with 8 time points at 11.8, 17.7, 23.6, 29.4, 35.3, 41.2, 47.1, $53.0 \mathrm{~ms}$; repetition time $6000 \mathrm{~ms}$; matrix size 256x256. Volume resonator body $150 \mathrm{~mm}$ coil was used for all relaxivity measurements. Signal intensities were measured using a predetermined square region of interest with ImageJ.

Larger clot target was necessary in order to easily visualize contrast enhancement. This was assessed using fibrin clots that were synthesized as described above. The clot was cut into uniform portions of approximately $0.5 \mathrm{~cm}$ in diameter and embedded in $0.6 \%$ agarose gel. As above, triplicate samples were incubated with Gd(III)-FA-NOTA for 48- 
hours, after which T2-weighted scans ( 7 Tesla, echo time $=42 \mathrm{~ms}$, repetition time $\geq 6 \mathrm{~s}$ ) were performed.

\subsubsection{In vitro samples (rat blood)}

Whole rat blood was clotted using a previously established thrombectomy protocol. ${ }^{152}$ In brief, $1 \mathrm{~mL}$ of $\mathrm{CaCl}_{2}$-thrombin solution was prepared using $0.252 \mathrm{M} \mathrm{CaCl}_{2}$ and $100 \mathrm{U}$ of thrombin. This solution was subsequently mixed with $5 \mathrm{~mL}$ of whole blood and left to form a clot at room temperature for 30 minutes. The clot was sectioned into slices approximately $0.5 \mathrm{~cm} \times 1 \mathrm{~cm}$ in size and combined with heparinized blood pool. Each sample was combined with $500 \mu \mathrm{L}$ of the $50 \mu \mathrm{M}$ Gd(III)-NOTA-FA contrast to a total blood pool volumes of $2 \mathrm{~mL}$. Both T1- and T2-weighted scans (echo time $=7.3 \mathrm{~ms}$, repetition time $=500 \mathrm{~ms}$, and echo time $=42 \mathrm{~ms}$, repetition time $\geq 6000 \mathrm{~ms}$, respectively) were performed over a 45-minute span. Volume resonator body $150 \mathrm{~mm}$ coil was used for all imaging. Signal intensities of both T1- and T2-weighted scans were measured using a square ROI with ImageJ software.

\subsection{Results and discussion}

\subsubsection{Fibrinogen aptamer binding validation}

\subsubsection{Fluorescence microscopy}

FA was previously selected by $\mathrm{Li}$ et al and showed good affinity towards fibrinogen, with a dissociation constant of $64 \mathrm{nM} \cdot{ }^{28}$ To assess the feasibility of FA to specifically bind the polymerized fibrin network, FA was tagged with a green emitting fluorophore, 6-fluorescein phosphoramidite (6-FAM), and applied directly to a thinly sliced synthetic fibrin clot. After washing away unbound aptamer with excess PBS post a 
30-minute incubation period, co-localization events were expected to result in the development of green fluorescence, the intensity of which was measured with fluorescence microscopy. A previously reported toxin-binding aptamer, FB139, was also modified with 6-FAM and used as a negative control due to its similar length and predicted secondary structure characteristics. ${ }^{169}$ This workflow is outlined in Figure 3.1A. When imaged under the blue channel at an excitation wavelength of $355 \mathrm{~nm}$, fibers within the fibrin network exhibited blue fluorescence, which was preserved through incubations with 6-FAM FB139 and 6-FAM FA. Under the green channel at excitation wavelength of $480 \mathrm{~nm}$, some dull green colour was present in the fibrin protein incubated with PBS, pH 7.4 (Figure 3.1B, $1^{\text {st }}$ row). This colour was attributed to background from the light-emitting diode of the microscope. Weak green fluorescence was present post incubation with 6-FAM FB139 (Figure $3.1 \mathrm{~B}, 2^{\text {nd }}$ row). This indicates a small amount of non-specific binding of the aptamer, likely based on the porous nature of the fibrin clot slice. Binding of 6-FAM FA with fibrin protein was verified by the presence of intense green fluorescence (Figure 3.1B, $3^{\text {rd }}$ row).

To determine whether FA binding to fibrin is selective, the observed differences in fluorescence were quantified using the ImageJ analysis program and compared (Figure 3.1C). This was done using the ratio of green to blue fluorescence intensities in order to account for fibrin's intrinsic blue fluorescence. Using One-way ANOVA statistical analysis, average pixel intensities (API) of each of the samples were compared revealing a statistically significant difference between the treatments $(F(2,27)=189.9, p<0.001$ at $\alpha=$ 0.01). As above, some background green colour was present in the fibrin incubation with PBS, pH 7.4, while incubation with 6-FAM FB139 aptamer resulted in weak green 
fluorescence. When compared to one another with Post-hoc Tukey HSD test, with APIs measuring at $0.34( \pm 0.07)$ and $0.44( \pm 0.04)$, respectively, there was no statistical difference between the two samples $(\mathrm{p}=0.011)$. Comparing the fibrin control and fibrin incubated with 6-FAM FB139 to fibrin incubated with 6-FAM FA, API measuring at $0.94( \pm 0.10)$, revealed that green fluorescence more than doubled. There was a statistical difference between both the measurements of fibrin control and fibrin incubated with 6-FAM FA $(\mathrm{p}<0.001)$, as well as fibrin incubated with 6-FAM FB139 and fibrin incubated with 6FAM FA ( $<<0.001)$. From this, it can be inferred that FA shows affinity for fibrin, beyond any non-specific binding stemming from the oligonucleotide structure, even though FA was not selected to bind fibrin protein. This implies that the polymerized structure must be such that there are enough sites for FA to be able to bind to structural features originating from fibrinogen monomers. 


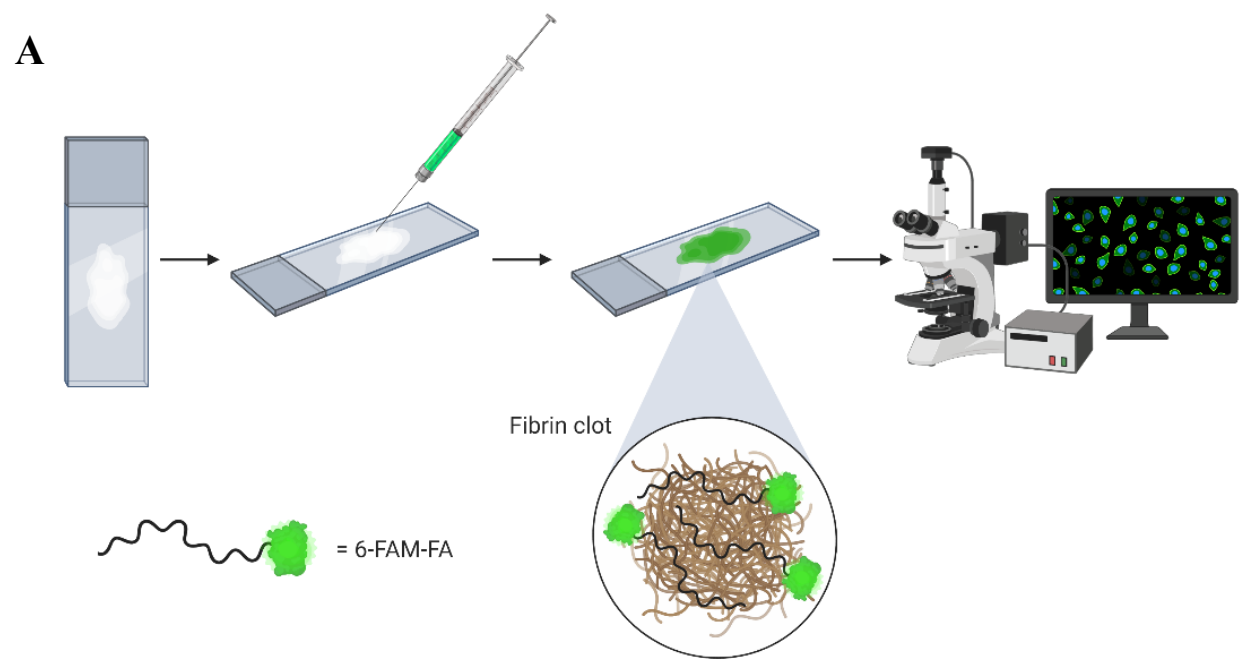

B

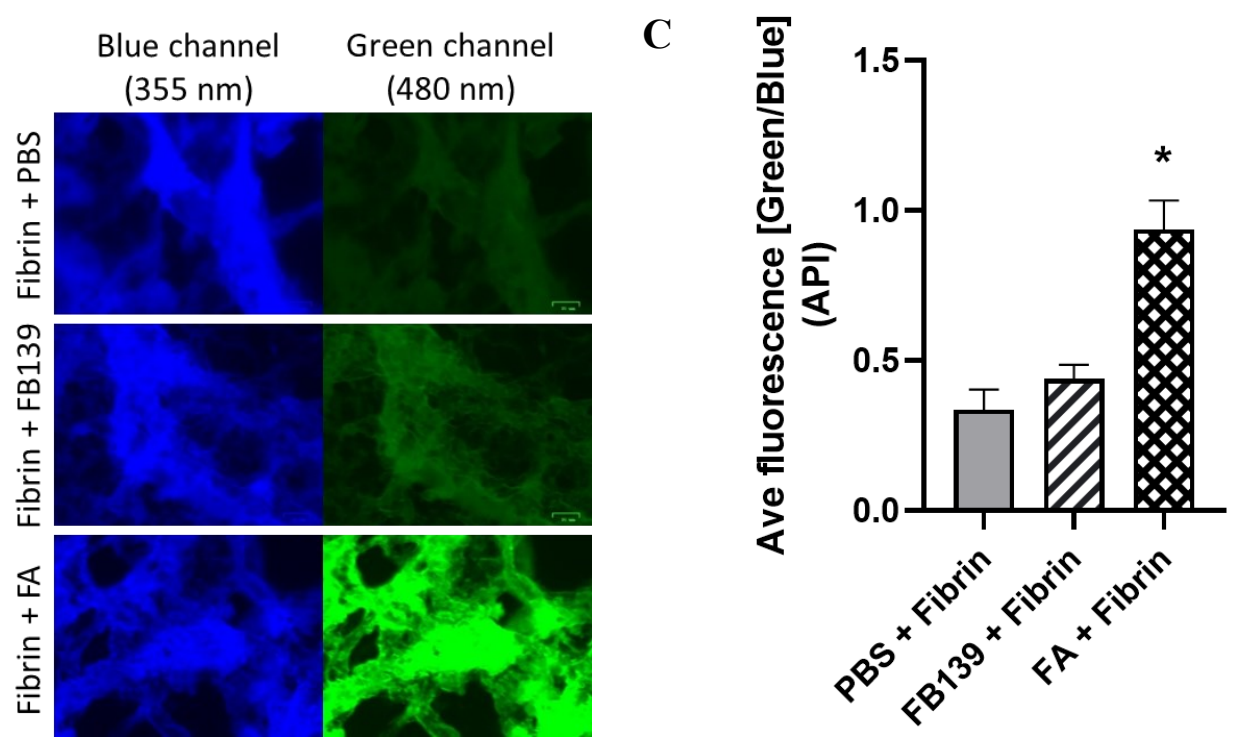

Figure 3.1: (A) Schematic representation of sample preparation for fluorescence microscopy, made in BioRender; (B) fluorescence microscopy images, under blue and green channels, with excitation wavelengths of $355 \mathrm{~nm}$ and $480 \mathrm{~nm}$, respectively: ( $\left(1^{\text {st }}\right.$ row $)$ fibrin incubated with PBS, $\mathrm{pH} 7.4,\left(2^{\text {nd }}\right.$ row $)$ fibrin incubated with 6-FAM FB139, (3 ${ }^{\text {rd }}$ row) fibrin incubated with 6-FAM FA; (C) Average ratios of green to blue fluorescence of control, 6-FAM FB139, and 6-FAM FA, measured by ImageJ Software and compared using One-way ANOVA statistical analysis $(F(2,27)=189.9, p<0.0001)$, Tukey post-hoc analyses revealed a significant difference between groups $\left({ }^{*} \mathbf{p}<0.01\right)$. Significance is indicated by *. 
Additional experiments were performed using the fluorescence microscopy method in order to determine the kinetics for the interaction between FA and fibrin protein. Thin slices of fibrin were incubated with 6-FAM FA for various timespans, ranging from 30 seconds, up to 30 minutes and imaged subsequent to washing away unbound aptamer with excess PBS. Under an excitation wavelength of $480 \mathrm{~nm}$, weak green fluorescence could be seen as soon as 30 seconds (Figure S3.1A, $1^{\text {st }}$ column), and its intensity increased dramatically by 10 minutes (Figure S3.1A, $4^{\text {th }}$ column). There was no observable difference in the amount of green fluorescence intensities measured at 30 seconds, 1 minute, and 5 minutes (Figure S3.1A, columns 1-3). Based on these results, it can be inferred that the binding of 6-FAM FA upon interaction with fibrin protein is almost immediate, but in order for a substantial number of binding events to occur, resulting in intense green fluorescence, approximately 10-minute incubation is required.

In order to confirm the visually observed trend, API of each of the time-points was measured by ImageJ (Figure S3.1B). When comparing average fluorescence of 30-second, 1-minute, and 5-minute incubations, it is evident that there is no significant difference in those measurements, while fluorescence doubles post 10-minute incubation, and again doubles that post 30-minute incubation. One-way ANOVA analysis verified that there was a statistical difference between those samples $(F(4,23)=148.0, p<0.001$ at $\alpha=0.01)$. Posthoc Tukey HSD test confirmed the observed trend, showing no statistical difference between 30-second and 1-minute incubations ( $\mathrm{p}=0.90), 1$-minute and 5-minute incubations $(\mathrm{p}=0.65)$, and 30 -second and 5 -minute incubations $(\mathrm{p}=0.52)$. There was a significant difference between 10-minute, and 30-minute incubations $(\mathrm{p}<0.001)$, and both of those were significantly different from the lower time-measurements, all compared pairwise 
$(\mathrm{p}<0.001)$. This type of kinetic study can be directly related to a real MRI scan, using an IV injection of a contrast agent. In a typical scan, contrast is circulated throughout the body within seconds, and each scan lasts approximately $10-15$ minutes. ${ }^{147,172}$ In an MRI scan that were to use aptamer-targeted contrast agent, given the same timescale, FA would have enough time to reach the location of the blood clot and accumulate, providing targeted enhancement.

The above fluorescent experiments demonstrate localization of 6-FAM FA within the fibrin network, which confirms the ability of FA to interact with and bind to fibrin protein. This binding is specific, as confirmed by the lack of fluorescence development when using 6-FAM FB139 as a negative control. Any binding of the negative control aptamer with fibrin was insignificant and attributed to non-specific interactions based on fibrin's porous nature (Figure 3.1). When incubated directly with the fibrin clot slices, 6FAM FA is able to bind to its target within minutes, which was demonstrated by the fluorescent time-study. This binding becomes more dramatic as incubation times increase and more binding events occur (Figure S3.1). These binding confirmation experiments allowed for streamlining in the development of fibrin-specific gadolinium-based MRI contrast agent. All in vitro assays were designed to mimic physiological conditions maintaining the $\mathrm{pH}$ at 7.4 to ensure that the final aptamer-contrast formulation can be directly translated into in vivo testing.

\subsubsection{Synthesis and assessment of fibrinogen aptamer contrast conjugates}

Literature precedent demonstrates the utility of different formulations of aptamercontrast complexes. Lu group has developed a "smart" contrast agent in which adenosinebinding aptamer was modified with streptavidin and hybridized with a complementary 
strand carrying Gd(III)-DOTA. Upon binding with adenosine, the aptamer strand would dissociate from the complementary strand, thereby releasing the Gd(III)-DOTA and resulting in a $34 \%$ decrease of relaxivity, a "turn off" response. ${ }^{173}$ Another example of a targeted contrast agent was developed by the same group. In this case, superparamagnetic iron oxide nanoparticles were functionalized with a thrombin aptamer and were used to adjust $\mathrm{T} 2$ relaxation time. Interaction with the thrombin target resulted in aggregation of iron oxide nanoparticles thus reducing the $\mathrm{T} 2$ relaxation time and resulting in darkening contrast. $^{174}$ Our own group has reported a formulation which led to a more clinically favourable "turn on" response, using thrombin-binding aptamer conjugated with GdDTPA. Upon binding with thrombin, relaxivity was improved by $20-35 \%$. From these and multiple other examples, it has become evident that targeted biomaterials allow for more defined interactions within biological systems. ${ }^{88,89,175-177}$ In this study, DOTA and NOTA chelators were chosen as they have been shown to offer more optimal longitudinal relaxation rates, while exhibiting good thermodynamic stability of $\log \mathrm{K}_{\mathrm{Gd}-\mathrm{DOTA}}=24.7$ and $\log \mathrm{K}_{\mathrm{Gd}-\mathrm{NOTA}}=14 \cdot 3 \cdot{ }^{178-180}$

To facilitate the conjugation with macrocyclic bifunctional chelators, used for complexing of the gadolinium ion, the aptamer was initially functionalized at the 5 '-end with a hexyldisulfide group which ensured that the chelator is incorporated using a singular reaction site in a two-step process. This controls for the position and the precision of subsequent complexation, avoiding undesirable side-products. ${ }^{109}$ First, the hexyldisulfide group was reduced with the help of dithiothreitol, a redox agent, to yield a free reactive thiol group. ${ }^{111}$ Maleimido-monoamide-DOTA or -NOTA macrocyclics were subsequently reacted in excess to ensure full incorporation (Scheme S3.1). ${ }^{109}$ This provided a built-in 
spacer between the oligonucleotide and the chelator, offering an opportunity to minimize steric hindrance effects to the structure and function of the aptamer. ${ }^{109}$ Excess chelator molecules were removed by size-exclusion to avoid unconjugated complexation with excess macrocyclics. DOTA- and NOTA-FA were quantified with a yield of $89 \%$ and $93 \%$, respectively, and the mass was confirmed by ESI mass spectrometry with molecular ion peaks of 28247.0 $\mathrm{Da}$ and 28145.6 $\mathrm{Da}$, respectively. A similar method of aptamer conjugation was reported by Gijs et al. ${ }^{109}$ These aptamer conjugates were subsequently loaded with Gd(III), using a 1:1 molar equivalent in an overnight reaction. To avoid the association of $\mathrm{Gd}(\mathrm{III})$ with the negatively charged DNA backbone, charge saturation was achieved using sodium ions present in the Tris buffer $(140 \mathrm{mM}) .{ }^{14}$ Unchelated Gd(III) was isolated from the conjugate, with each aptamer strand bearing a single chelator complexed with a single Gd(III) ion, and the content was measured using a xylenol orange test. Xylenol orange, a complexometric dye, is a common indicator used to assess lanthanide(III) ion content in a solution, as it forms coloured 1:1 complexes. A calibration curve was constructed using the absorbance of varying amounts of $\mathrm{Gd}(\mathrm{III})$, while the amount of the dye was kept constant (Figure S3.4). The same amount of the dye was added to the excess salts collected from the washes of Gd(III)-DOTA- or Gd(III)-NOTA-aptamer. The absorbance of these solutions was used to determine the content of free $\mathrm{Gd}(\mathrm{III})$ utilizing the formula obtained by the trendline of the calibration curve (Figure S3.5). ${ }^{114}$ Percent loading was then calculated, using the amount of free $\operatorname{Gd}(\mathrm{III})$ and the starting amount as above. Loading efficiency was determined to be $89 \%$ for the Gd(III)-DOTA-FA conjugates and $91 \%$ for the Gd(III)-NOTA-FA conjugates. Final Gd(III)-DOTA-FA and Gd(III)-NOTA-FA conjugates were characterized using mass spectrometry with molecular 
ion peaks at 28403.1 $\mathrm{Da}$ and 28298.1 $\mathrm{Da}$, respectively, representing complete loading. If loading efficiency was determined to be unacceptable, with free $\mathrm{Gd}(\mathrm{III})$ exceeding $10 \%$, the chelation process was repeated. The same procedure was repeated in the production of the control aptamer conjugate.

After synthesis and loading, the ability of Gd(III)-NOTA-FA to produce significant contrast was assessed at 7 and 9.4 Tesla. It is common to describe the efficiency of a contrast agent in terms of relaxivity, which is the increase of the rate of relaxation of nearby water protons as a function of the concentration of paramagnetic material (as described in equation 2.7, Chapter 2). Relaxivities of Gd(III)-NOTA-FA and Gadovist, a clinical GdDO3A agent, were compared and calculated to be $6.72 \mathrm{mM}^{-1} \mathrm{~s}^{-1}$ and $8.57 \mathrm{mM}^{-1} \mathrm{~s}^{-1}$ at $9.4 \mathrm{~T}$ and $\mathrm{pH}$ of 7.4, respectively (Figure 3.2). While accounting for reported decrease of relaxivity with increasing frequency, the measurement obtained for Gadovist is comparable to that reported in literature in the range of $100 \mathrm{MHz} \cdot{ }^{178,181,182}$ Notably, relaxivity of Gd(III)-NOTA-FA was found to be lower at the same frequency. This may be related to the presence of a long oligo strand, conjugated to the chelator. In particular, FA was denatured and re-folded to obtain the most optimal secondary structure, and through this process, it is possible the steric hindrance effects were enhanced. The overall increased size of the final formulation likely altered the water exchange path, resulting in steric blocking and, therefore, decreasing the water exchange time. ${ }^{183}$ Nevertheless, Gd(III)-NOTA-FA displayed the proper trend of increasing contrast enhancement with increasing concentration of the paramagnetic ion. This is due to the ligand denticity of NOTA, which allows for $q$ (hydration number) to be 2 , resulting in a greater number of rapidly exchanging water molecules to be directly coordinated to $\mathrm{Gd}(\mathrm{III})$, contributing to "inner 
sphere" and therefore observable relaxation. ${ }^{183,184}$ To further support the hypothesis of steric bulk having a measurable effect in this type of formulation, relaxivity associated with Gd(III)-NOTA-FA bound to the fibrin target was calculated. It was found that relaxivity of Gd(III)-NOTA-FA decreased from $6.72 \mathrm{mM}^{-1} \mathrm{~s}^{-1}$ to $4.54 \mathrm{mM}^{-1} \mathrm{~s}^{-1}$ at $9.4 \mathrm{~T}$ and $\mathrm{pH}$ of $7.4 \mathrm{in}$ the presence of the fibrin target at 1000:1 ratio (Figure 3.2). Since fibrin is a large protein, and FA binding could lead to the incorporation of the 5' tail into its structure (discussed in Chapter 4), it is possible that Gd(III)-NOTA, which is conjugated in that region, is also incorporated upon binding. This would inherently expose Gd(III)-NOTA-FA to a different surrounding, changing relaxivity. ${ }^{183,184}$

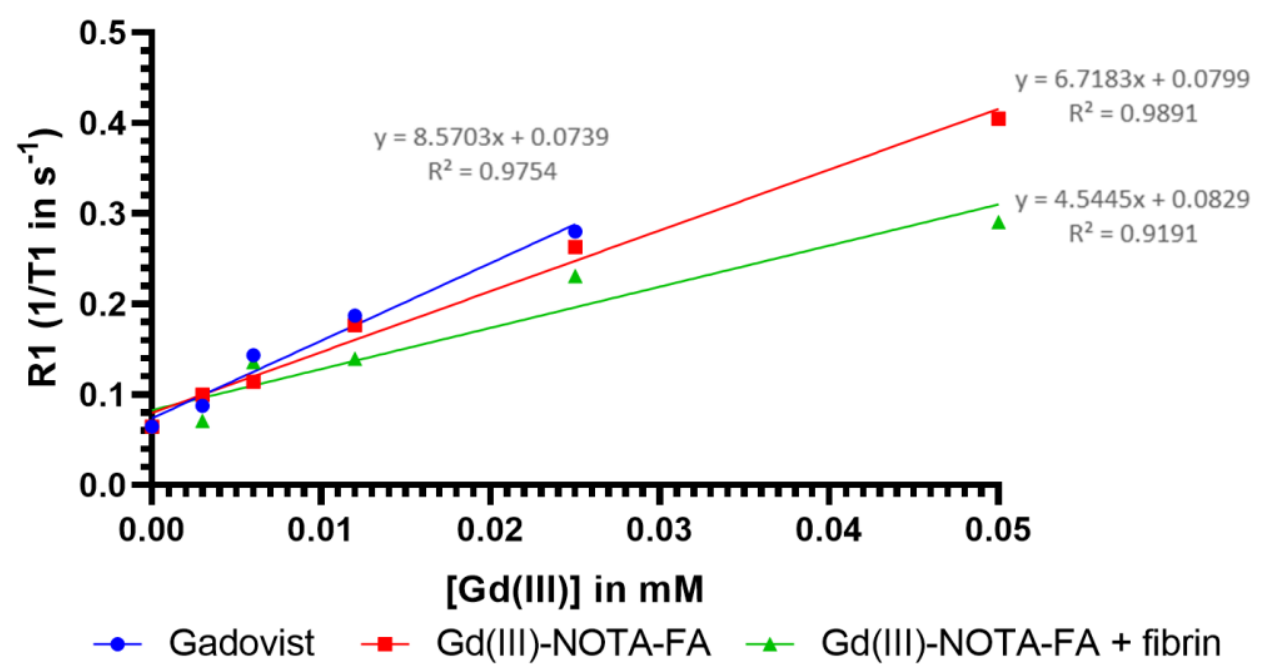

Figure 3.2: Longitudinal relaxivity plots of Gadovist, Gd(III)-NOTA-FA, and Gd(III)-NOTA-FA incubated with fibrin at 1000:1 ratios, done at 9.4 $\mathrm{T}, \mathrm{pH}$ 7.4.

Measuring relaxivity of Gadovist, Gd(III)-NOTA-FA and the bound complex of Gd(III)-NOTA-FA with fibrin using a clinical MRI scanner (7 T) allowed for comparison of the contrast efficiency at a different frequency. The same trend of decreasing relaxivity was observed, with calculated values of $4.85 \mathrm{mM}^{-1} \mathrm{~s}^{-1}, 3.04 \mathrm{mM}^{-1} \mathrm{~s}^{-1}$, and $1.73 \mathrm{mM}^{-1} \mathrm{~s}^{-1}$ for 
Gadovist, Gd(III)-NOTA-FA, and the bound complex with the fibrin target (Figure 3.3). That is, the relaxivity of Gd(III)-NOTA-FA was determined to be $37 \%$ lower than that of Gadovist, while an additional decrease of $43 \%$ was observed in the presence of the fibrin target. These results further support that the observed changes are not exclusive to the magnetic field strength and are related to the structure and characteristics of the contrast material and the target used.
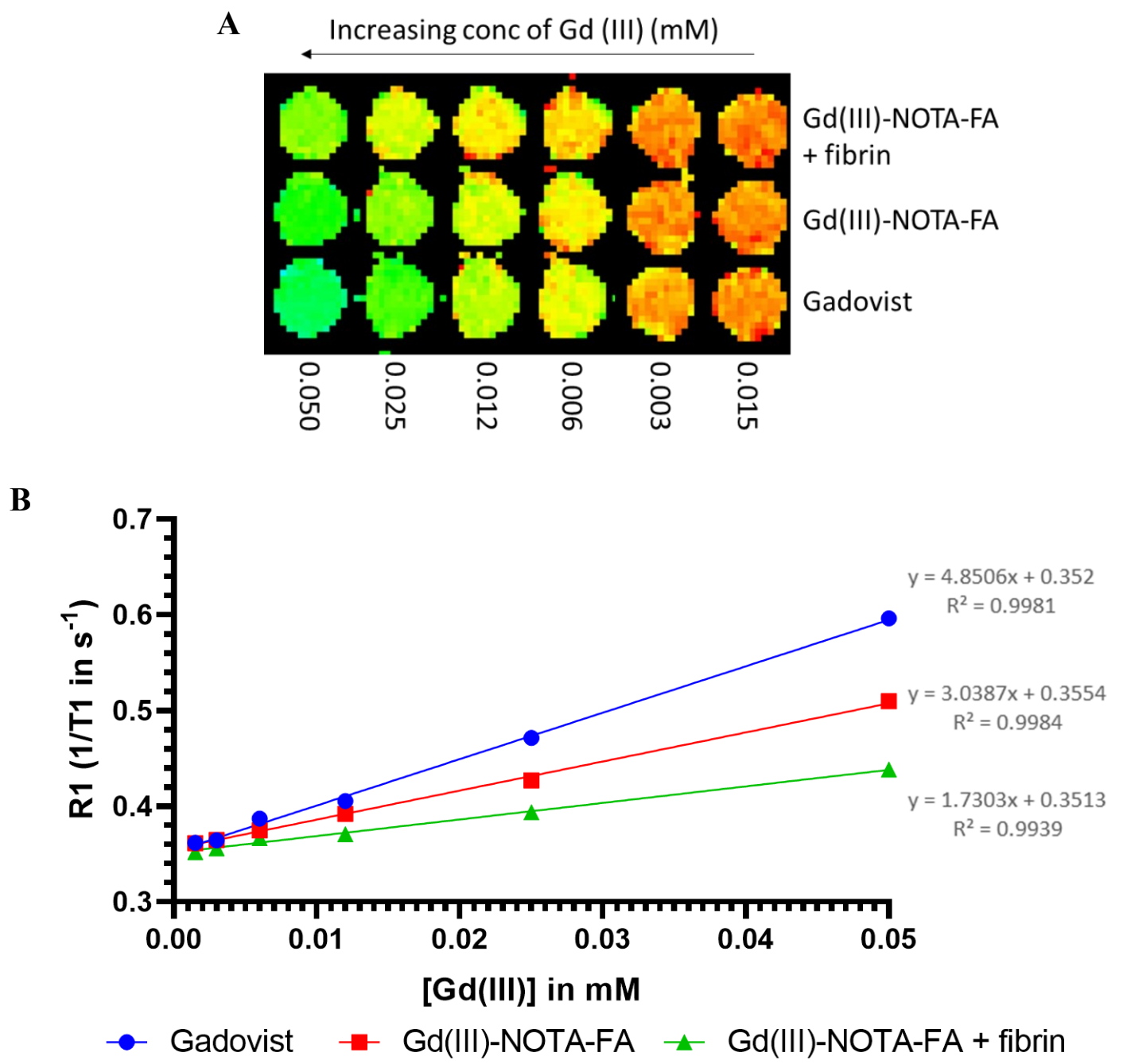

Figure 3.3: (A) T1 map MR images of over an increasing range of concentrations of Gadovist, Gd(III)NOTA-FA, and Gd(III)-NOTA-FA + fibrin; (B) Longitudinal relaxivity plots of Gadovist, Gd(III)NOTA-FA, and Gd(III)-NOTA-FA incubated with fibrin at 1000:1 ratios, done at 7 T, pH 7.4. 
With the trend of decreasing $\mathrm{T} 1$ relaxivity, it was essential to assess whether $\mathrm{Gd}(\mathrm{III})-\mathrm{NOTA}-\mathrm{FA}$ is being converted into a more effective $\mathrm{T} 2$ agent. T2 contrast agents achieve their effect through the interaction between excited nuclei and those at a lower energy through energy transfer (as discussed in Chapter 2). It has been previously reported that $\mathrm{Gd}(\mathrm{III})$ is able to enhance both $\mathrm{T} 1$ and $\mathrm{T} 2$ relaxation rates, given a concentration that is high enough to overwhelm the $\mathrm{T} 1$ effects. ${ }^{183}$ This, in turn, has been explored in examples such as optimization of the contrast-to-noise ratio during cholangiopancreatography. ${ }^{159}$ When measured at 7T, $\mathrm{T} 2$ shortening effects of Gadovist appeared to be comparatively negligible, with a measured relaxivity of $8.17 \mathrm{mM}^{-1} \mathrm{~s}^{-1}$. However, both unbound $\mathrm{Gd}(\mathrm{III})-$ NOTA-FA and bound Gd(III)-NOTA-FA (+fibrin) exhibited increased relaxivity, with measured values of $38.7 \mathrm{mM}^{-1} \mathrm{~s}^{-1}$ and $46.5 \mathrm{mM}^{-1} \mathrm{~s}^{-1}$, respectively (Figure 3.4). This can be explained by the principle of outer-sphere water relaxation having an effect on T2 relaxation. As previously, it would be reasonable to speculate that when Gd(III)-NOTA is bound to FA, inner-sphere contributions are decreased. With that, outer-sphere effects dominate, translating into $\mathrm{T} 2$ relaxivity that is nearly 5-times higher than unconjugated contrast agent. This effect is further enhanced by an additional $17 \%$ in the presence of the target, as binding may lead to further intercalation of the Gd(III)-NOTA chelator. ${ }^{157,159}$ 


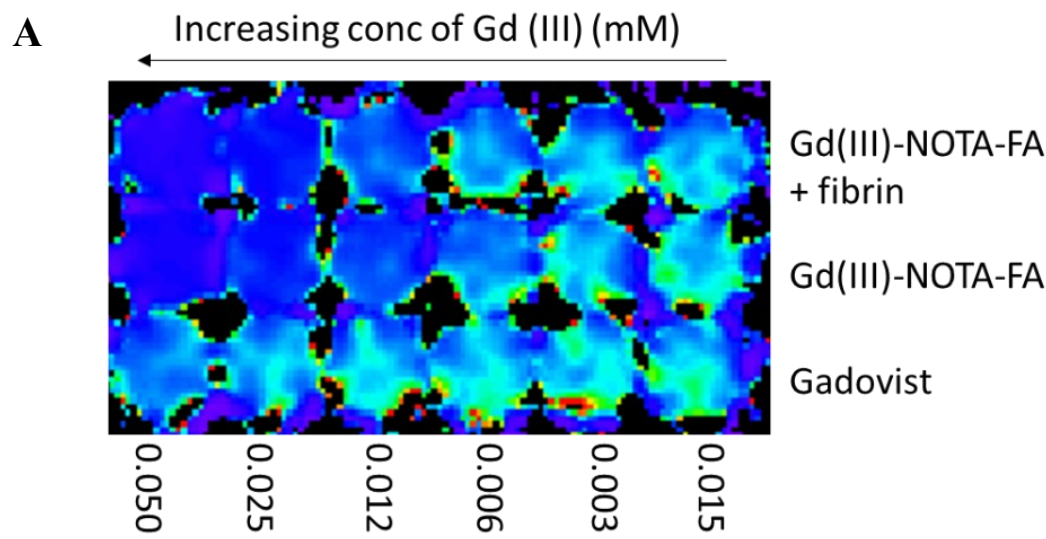

\section{B}

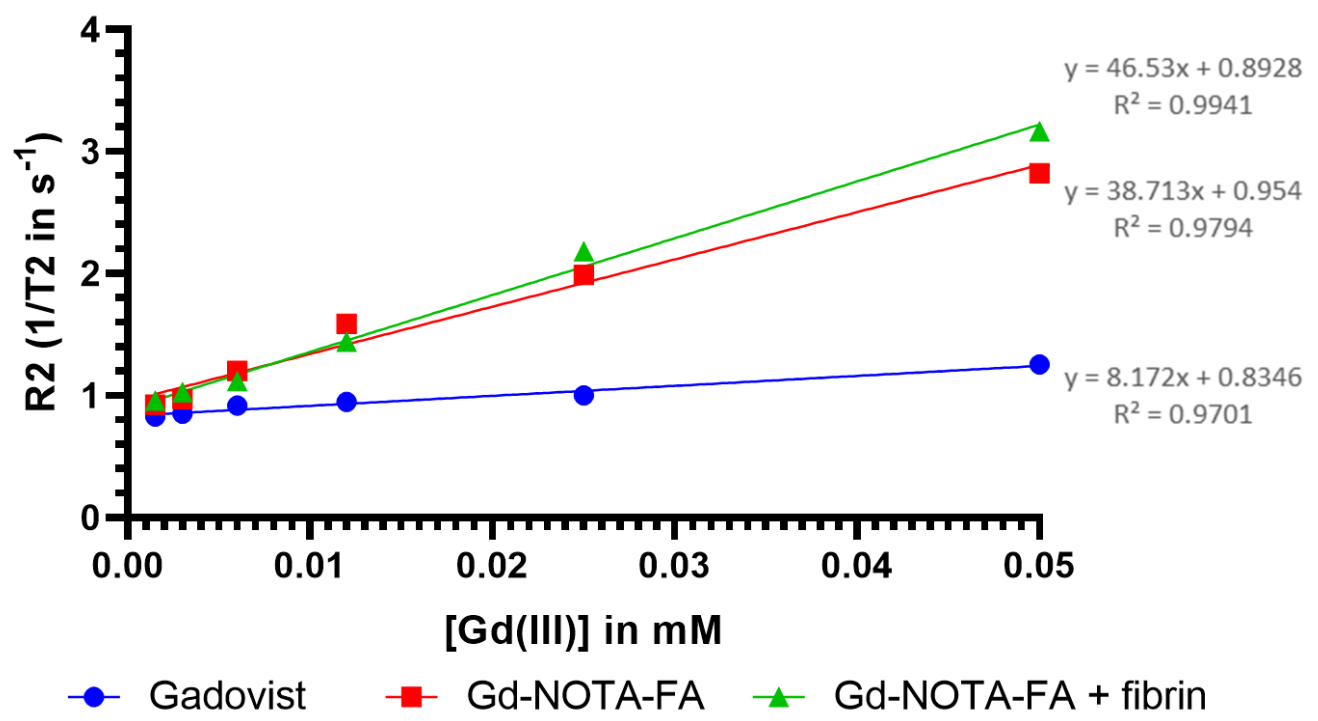

Figure 3.4: (A) T2 map MR images of over an increasing range of concentrations of Gadovist, Gd(III)NOTA-FA, and Gd(III)-NOTA-FA + fibrin; (B) Transverse relaxivity plots of Gadovist, Gd(III)NOTA-FA, and Gd(III)-NOTA-FA incubated with fibrin at 1000:1 ratios, done at $7 \mathrm{~T}$, pH 7.4.

\subsubsection{Microscale thermophoresis}

Since FA was originally selected against human fibrinogen, it was expected that a certain proportion of Gd(III)-NOTA-FA would bind the fibrinogen within the blood pool. ${ }^{28}$ To account for this, the dissociation constants $\left(\mathrm{K}_{\mathrm{d}}\right)$ associated with the binding of FA to fibrinogen and fibrin were determined by microscale thermophoresis. Since the $\mathrm{K}_{\mathrm{d}}$ for fibrinogen was previously determined and reported by Li et al., it served as a milestone for 
that of fibrin. ${ }^{28}$ First, FA was analyzed with the fibrinogen target and fluorescence enhancement effect was observed, with increasing concentration of the protein leading to increased fluorescence. The fluorescence time-trace demonstrates a plateau and the lowest and the highest concentrations of the protein, with a linear dynamic range present in the middle (Figure 3.5A). Notably, this fluorescence enhancement may be rooted in alterations of solute properties of the FA-fibrinogen complex or intercalation of a given region of the protein into the aptamer strand. ${ }^{185,186}$ The latter would appear to be reasonable, as the aptamer strand chosen for this work was predicted to bind away from the $\beta$ and $\gamma$ chains, likely gravitating towards the coiled-coil regions. In addition, fluorescence enhancement may arise from binding-induced change in the environment surrounding the $\mathrm{Cy} 5$ modifier. That is, restricted isomerization of the five carbon polymethine bridge as a consequence of increased viscosity within the fibrin network would lead to higher fluorescence. ${ }^{187}$ The $\mathrm{K}_{\mathrm{d}}$ was calculated to be $89.6 \mathrm{nM}$ (average of two technical runs). This aligned with the previously reported $\mathrm{K}_{\mathrm{d}}$ of $64 \mathrm{nM}$ and confirmed that this method of assessment can be used for this binding pair. Next, FA was analyzed in the presence of fibrin. Again, in the presence of increasing concentrations of the protein, fluorescence enhancement effect was observed. Since fibrin is a polymer of fibrinogen, this was expected and indicates that the binding of FA to fibrin is similar to that of fibrinogen. The fluorescence time-trace was used to confirm this hypothesis, with a plateau present at the lowest and highest concentrations, and a linear dynamic range in the middle (Figure 3.5B). The apparent $\mathrm{K}_{\mathrm{d}}$ (based on the concentration of fibrinogen constituents attained through UV-Vis) was calculated to be $16.6 \mathrm{nM}$, demonstrating avidity as the result of the presence of multiple closely spaced binding sites (receptor clustering). That is, since fibrinogen monomers 
remain largely unchanged through the polymerization process, this provides a larger number of binding sites for FA, having a positive effect on binding affinity. ${ }^{26}$

A

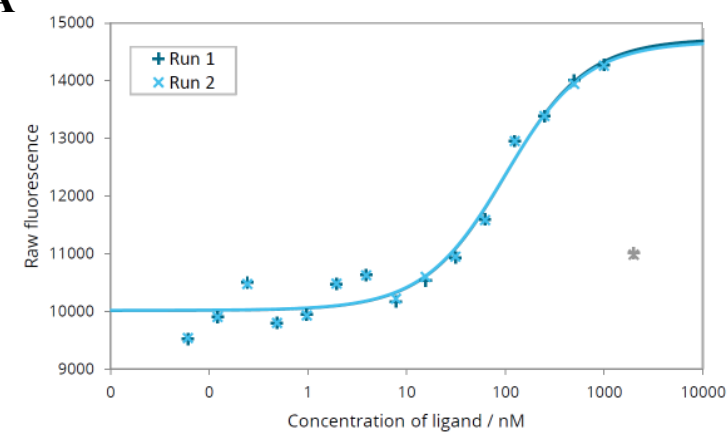

B

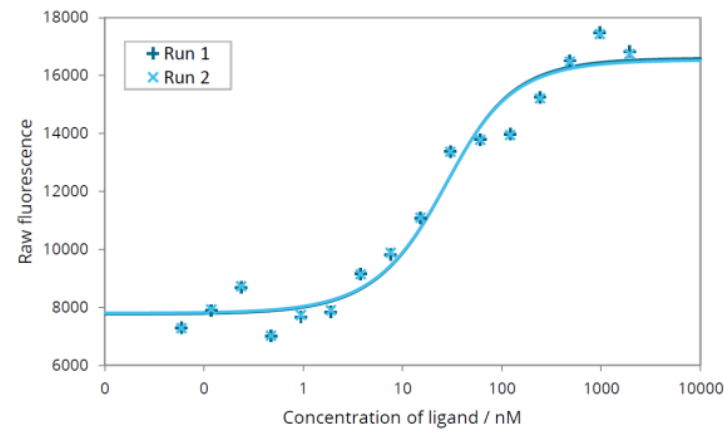

Figure 3.5: Microscale thermophoresis analysis for: (A) FA in the presence of increasing concentrations of fibrinogen, and (B) FA in the presence of increasing concentrations of fibrin. Two technical runs were performed, and the constructed S-curve was used in determination of dissociation constants.

\subsubsection{Phantom MRI studies}

Phantom MRI studies were performed at $7 \mathrm{~T}$, using lyophilized fibrin powder from human plasma. Both Gd(III)-DOTA-FA and Gd(III)-NOTA-FA conjugates were tested in order to optimize the system and determine which aptamer contrast agent would be the most promising for subsequent testing in biological samples. As above, FB139 was used as the negative aptamer control, while collagen was used as the negative protein control. Collagen was chosen based on its similarity in size and charge to fibrin. ${ }^{188-190}$ Agarose gel was used based on its ability to mimic tissue density and provide a bright background while avoiding undesirable artifacts in an MR scan. ${ }^{191}$ All samples were scanned post a 48-hour incubation (Figure 3.6A), as determined to be the optimal wait time for the aptamer conjugate to diffuse into the $0.6 \%$ agarose gel. Briefly, fibrin was embedded into the agarose gel, depositing 6-FAM FA on top. Fluorescence development was monitored over 
a 48-hour time period as the aptamer diffused through the system (Figure S3.3). To understand the effect of Gd-DOTA-FA or Gd(III)-NOTA-FA target binding, T2 map images were assessed visually, and by relaxivity values were determined. Sample darkening, associated with treatments with $\mathrm{Gd}(\mathrm{III})-\mathrm{DOTA}-\mathrm{FA}$ or $\mathrm{Gd}(\mathrm{III})-\mathrm{NOTA}-\mathrm{FA}$ revealed that gadolinium may be more effective as a T2 agent, when it is immobilized in a phantom gel system and bound to the target through the aptamer. This is reasonable as gadolinium requires proper molecular tumbling near the Larmor frequency in order to shorten spin-lattice relaxation times and produce bright contrast. Without this proper tumbling (or at high gadolinium concentration), dipole-dipole T2-shortening, and susceptibility effects dominate which leads to darkening. In a phantom system that is based on specific delivery of the CA to its target (consequences of target binding discussed above), mobile interactions will be limited due to immobilization of the contrast complex onto its respective target. ${ }^{192}$ In this scenario, Gd(III) shortens the spin-spin relaxation time (T2) of nearby protons. ${ }^{175}$

In order to determine if target binding elicits a significant change in relaxation rates, and if this change is selective in the presence of the appropriate target, image signal intensities were analyzed (Figure 3.6B). Samples were compared in two groups, fibrin incubations and collagen incubations, to account for slightly denser fibrous nature of collagen, yielding darker images overall (Figure 3.6A). Comparison of the measured transverse relaxation rates of each set of fibrin samples by One-way ANOVA test $(F(6,14)$ $=42.33, \mathrm{p}<0.0001$ at $\alpha=0.05)$ revealed a statistically significant difference between the treatments. Post-hoc Tukey HSD test was used for multiple pairwise comparisons. It was confirmed that there was no statistically significant difference in signal intensity between 
fibrin samples treated with Gd(III)-DOTA-FA and Gd(III)-NOTA-FA ( $\mathrm{p}=0.9832)$. There was, however, a significant difference between Gd(III)-DOTA-FA samples and fibrin PBS control ( $\mathrm{p}=0.0143$ ), and likewise between Gd(III)-NOTA-FA samples and fibrin PBS control ( $\mathrm{p}=0.0025$ ) (Figure 2B). Fibrin treated with Gd(III)-DOTA-FA or Gd(III)-NOTAFA yielded darker images and lower signal intensity, and both samples were significantly different from fibrin incubated with Gd(III)-DOTA-FB139 $(\mathrm{p}<0.01)$. Due to the increased density of the fibrin samples, phantoms that did not contain any target protein were only compared to show relaxation rates in the absence of target protein. The presence of fibrin target resulted in a significant decrease of relaxation times of both Gd(III)-DOTA-FA and Gd(III)-NOTA-FA, when compared to individual Gd(III)-DOTA-FA and Gd(III)-NOTAFA ( $<<0.01$ for each pairwise comparison). The same analysis was performed for each set of collagen samples, with One-way ANOVA test $(F(6,14)=27.02, p<0.0001$ at $\alpha=0.05)$ confirming that samples were significantly different. There was no significant difference between the image intensity of collagen PBS control, and collagen incubated with Gd(III)DOTA-FA ( $\mathrm{p}=0.9579)$, or collagen incubated with $\mathrm{Gd}(\mathrm{III})-\mathrm{NOTA}-\mathrm{FA} \quad(\mathrm{p}=0.4677)$. Moreover, there was no significant difference between collagen treatments with Gd(III)DOTA-FA and Gd(III)-DOTA-FB139 ( $\mathrm{p}=0.9953$ ), nor treatments with Gd(III)-NOTA-FA and Gd(III)-DOTA-FB139 ( $\mathrm{p}=0.9994)$. Importantly, the presence of collagen did not elicit a significant change in relaxation rates of neither $\mathrm{Gd}(\mathrm{III})-\mathrm{DOTA}-\mathrm{FA}(\mathrm{p}=0.3568)$ nor Gd(III)-NOTA-FA ( $p=8739$ ). From these results, it is evident that FB139 does not exhibit binding to fibrin or collagen, while FA does not bind to collagen. Furthermore, this confirms that Gd(III)-DOTA-FA and Gd(III)-NOTA-FA delivery system elicits significant enhancement in relaxation rates and is specific to its fibrin target, which supports the 
aptamer-target specificity that was demonstrated in the fluorescent experiments described above.

A

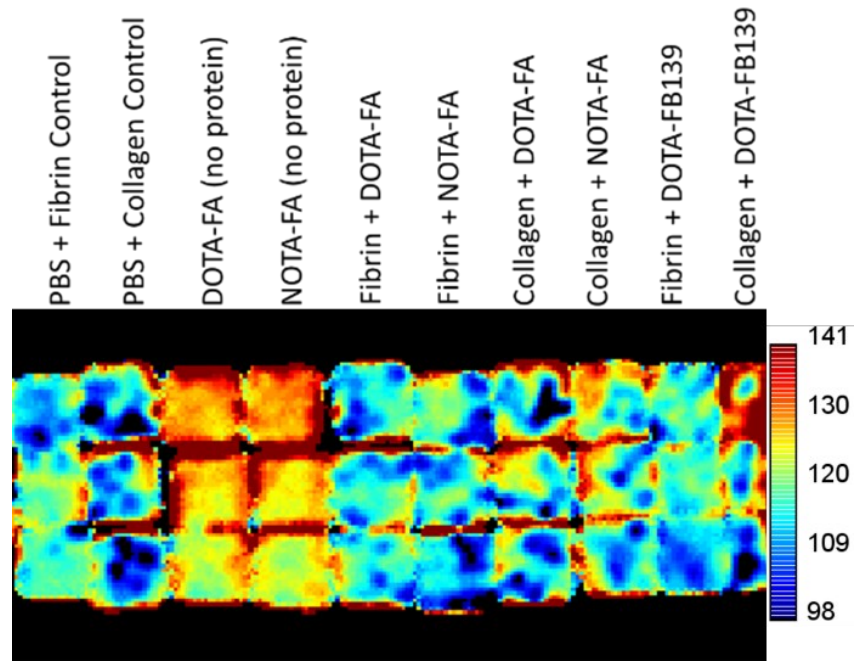

B

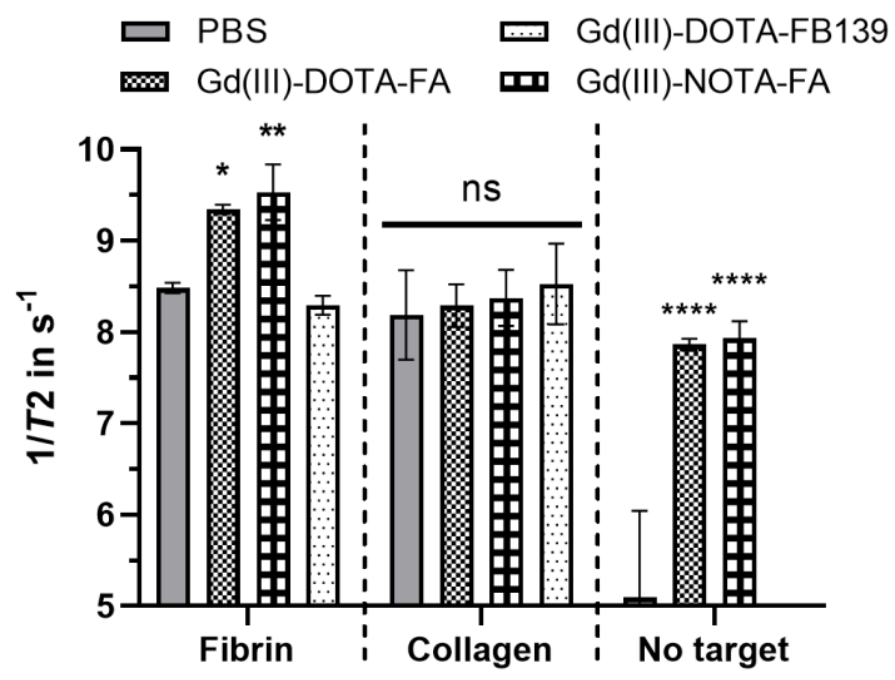

Figure 3.6: (A) T2 map MR images - fibrin and collagen proteins embedded in $0.6 \%$ agarose gel to produce phantom samples and imaged in triplicates, post incubation with Gd(III)-DOTA-FA or Gd(III)-NOTA-FA in PBS, pH 7.4 for experimental samples, and PBS, pH 7.4 or Gd(III)-DOTAFB139 for control samples for 48 hours; (B) Average relaxation rate measurements of each sample, acquired through T2-map sequence, and compared using One-way ANOVA test $(F(6,14)=42.33$, $\mathrm{p}<0.0001$ at $\alpha=0.05)$ and $(\mathrm{F}(6,14)=27.02, \mathrm{p}<0.0001$ at $\alpha=0.05)$; Tukey post-hoc analyses revealed a significant difference between treatments $(* p<0.05, * * p<0.01$, and $* * * * p<0.0001)$. 
From the optimized conditions that were determined through the experiments using lyophilized fibrin powder, a more clinically relevant model was examined. Gd(III)-NOTAFA was chosen as the more promising chelate for this study. T2-weighted images of a larger in-lab synthesized fibrin clot were acquired post a 48-hour incubation with Gd(III)NOTA-FA. As above, interaction between Gd(III)-NOTA-FA conjugate and the fibrin target resulted in darkening contrast. Analysis of each of the slices within the MRI series showed evidence of internal interaction of Gd(III)-NOTA-FA with the fibrin clot. This is demonstrated by a network of dark contrast present not only on the surface, but also throughout the clot, further verifying that FA is able to bind fibrin polymer (Figure 3.7). Some darkening is evident around the control sample of the clot incubated with PBS, $\mathrm{pH}$ 7.4 and is indicative of the presence of air pockets. These air pockets originate when the synthetic clot is inserted into agarose gel during the production of the phantom system. Since air pockets contain very little material, these appear as dark areas. ${ }^{193,194}$ Notably, no disruption of clot integrity was noted throughout any of the 48-hour incubations with FA. This indicates that, even though FA is able to penetrate the clot's surface and interact with the fibrinogen monomers internally, it does not perturb the network of the polymer. In a clinical setting, this would be important as the injection of FA-conjugate contrast media would not interfere with blood coagulation. 

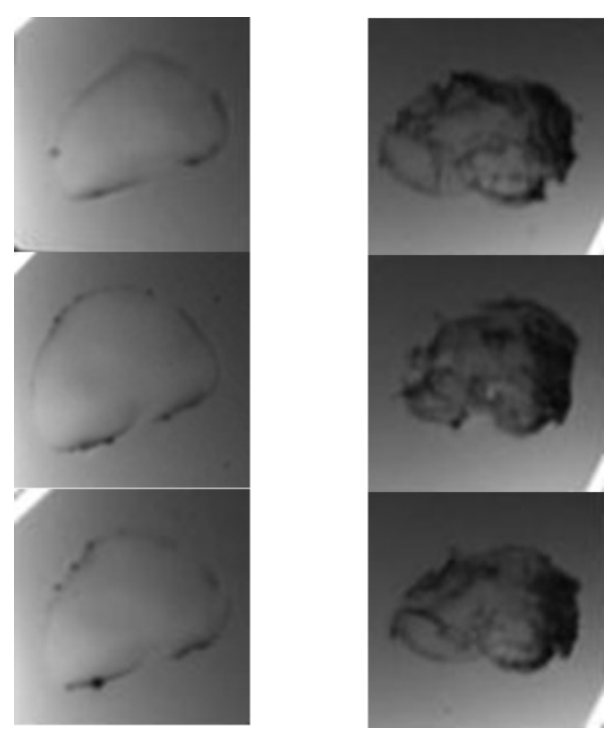

Figure 3.7: T2-weighted MR Image of fibrin clot incubated with PBS, pH 7.4 (left) and fibrin clot incubated with Gd(III)-NOTA-FA (right), post a 48-hour incubation. Different panels represent images taken through different depths of fibrin clot.

\subsubsection{In vitro MRI studies (rat blood)}

In vitro studies were conducted using clotted rat blood suspended in heparinized whole rat blood to accommodate for proper tumbling of gadolinium contrast as well as to better mimic a blood vessel environment (Figure 3.8A). Both T1- and T2-weighted scans were conducted on a similar time scale as the kinetic fluorescence studies during binding validation phase. These experiments were used to confirm that Gd(III)-NOTA-FA retains its binding affinity towards a more complex target, with blood cells trapped within the fibrin network, while suspended in a complex matrix. In addition, it was necessary to confirm that this binding still occurs on a timescale that would be clinically relevant. In a T2-weighted scan, both the whole blood pool and the blood clots appeared dark at the 0minute timepoint. Increasing signal intensity was observed at each acquired scan with considerable brightening of the blood clots at the 45-minute timepoint. The whole blood pool remained dark throughout (Figure 3.8B). On the other hand, in a T1-weighted scan, 
the opposite trend was observed. Namely, increasing signal intensity of the whole blood pool developed over a 45-minute time period, while the blood clots remained dark (Figure 3.8C). This enhancement was reasonable, as fibrinogen is one of the natural components of whole blood, circulating in plasma at concentrations of $160-400 \mathrm{mg} / \mathrm{dL} .{ }^{195}$ Since FA was selected against human fibrinogen, it was expected that some proportion of Gd(III)-NOTAFA would bind fibrinogen protein within the blood pool. ${ }^{28}$ In both scenarios, contrast development is based on target binding, which has shown to improve relaxivity of aptamergadolinium conjugates. ${ }^{14}$ The binding of Gd(III)-NOTA-FA to free fibrinogen increased the overall size and mass of the CA through the formation of the aptamer-target complex, likely resulting in decreased tumbling rates and thereby enhancing relaxivity. This may be associated with the effects of "receptor-induced magnetization enhancement" (RIME), bringing local magnetic fluctuation frequency closer to the Larmour frequency and increasing the effectiveness of the paramagnetic ion in T1-weighted scans. ${ }^{14,196}$ The overall result is an enhancement of signal of the blood pool in T1-weighted imaging. On the other hand, even though fibrin, which makes up the scaffold of blood clots, is composed of predominantly unchanged fibrinogen monomers, $\mathrm{T} 1$ enhancement is not observed at the location of blood clots. ${ }^{26,28}$ This is reasonable, as above described relaxivity studies determined that when Gd(III)-NOTA-FA is exposed to the fibrin target, longitudinal relaxivity decreases dramatically. When shifting the focus to T2-weighted imaging, it was expected that treatment with Gd(III)-NOTA-FA would result in the enhancement of transverse relaxation rates, and thus hypointense signal at the location of the clots. Unexpectedly, an increase in T2 signal intensity was observed, suggesting that transverse relaxation times are extended. This may be related to hydraulic permeability of the clot 
itself. Interstitial fluid flow is an important factor that is readily affected by the features of individual clots and permeability may vary up to 5 orders of magnitude. ${ }^{197}$ Since T2 outersphere effects rely on the movement of water protons near the local magnetic field gradient created by the paramagnetic center, diminished fluid flow may result in a lower number of available protons. This, in turn, prolongs transverse relaxation rates, yielding a higher signal intensity in T2-weighted images. ${ }^{183,198}$ The presence of the paramagnetic ion within the fibrous network, in this case, assists in enhancement of the clot in T2-weighted imaging. Importantly, this signal intensity increase indicates that Gd(III)-NOTA-FA is able to bind the fibrin network in the presence of blood cells within a blood clot and accumulate to produce observable enhancement, providing high resolution between the clot and surrounding blood. The combination of highly resolved clots in T2-weighted scans and highly resolved blood pool in T1-weighted scans can be used beneficially towards selectively labeling the location of blood clots. Instead of observing bright signal throughout the vasculature in a $\mathrm{T} 1$ scan, the acquisition time can be switched to T2, resulting in brightening of only blood clots. A notable advantage of this system is that a favourable "turn on" response is gained, while the duration of the scan is relevant to that of a typical MRI. This supports the hypothesis that an aptamer-gadolinium conjugate can be used to selectively deliver contrast to the intended target, allowing for a high-resolution scan. Importantly, binding of FA did not appear to disrupt the blood clot when observed up to 48-hours post incubation, indicating that aptamer-target binding does not interfere with the fibrin network (Figure S3.6).

Analysis of APIs of each of the samples at different time points by One-way Anova test $(\mathrm{F}(15,92)=104.8, \mathrm{p}<0.0001$ at $\alpha=0.01)$ suggested a statistically significant difference 
between the treatments (Figure 3.8D). In order to account for the difference in density of the materials, with blood clots being denser than the blood pool, blood clot treatments were compared separately from the blood pool treatments. In addition, there was no comparison across the acquisition times, as T1- and T2-weighted scans possess different characteristics. Post-hoc Tukey HSD test confirmed that, in a T2-weighted scan, the contrast associated with the density of the clots measured at 0 mins and contrast associated with $\mathrm{Gd}(\mathrm{III})$ NOTA-FA binding the clots at 45 mins was significantly different $(p<0.01)$. In the same scan, there was no significant contrast development within the blood pool from 0 mins to at 45 mins ( $\mathrm{p}=0.90$ ). In a T1-weighted scan, there was no significant contrast development at the location of the blood clots from 0 mins to $45 \operatorname{mins}(\mathrm{p}=0.42)$. However, the contrast development associated with Gd(III)-NOTA-FA binding to fibrinogen within the blood pool was significant at 45 mins $(p<0.01)$. Neither the blood pool, nor the blood clots developed any contrast when incubated with PBS as a control $(p=0.70$ and $p=0.90$, respectively). On the other hand, when treated with Gd(III)-NOTA-FB139, there was no contrast development over time and positive contrast was observed throughout the clots and within the blood pool. When compared to treatments with Gd(III)-NOTA-FA, it was evident that this contrast enhancement was non-specific (Table 3.3). This analysis confirms that the contrast development associated with $\mathrm{Gd}(\mathrm{III})$-NOTA-FA binding its target is significant and the conjugate can be used to produce highly resolved images. 
Table 3.3: Average pixel intensities of each in vitro sample in T1- and T2-weighted scans, indicating significant difference between one or more treatments

\begin{tabular}{|l|l|l|l|l|}
\cline { 2 - 5 } & $\begin{array}{l}\text { Blood pool } \\
(\mathbf{0} \text { mins })\end{array}$ & $\begin{array}{l}\text { Blood pool } \\
\mathbf{( 4 5} \text { mins })\end{array}$ & $\begin{array}{l}\text { Blood clots } \\
(\mathbf{0} \text { mins })\end{array}$ & $\begin{array}{l}\text { Blood clots } \\
(\mathbf{4 5} \text { mins })\end{array}$ \\
\hline $\begin{array}{l}\text { T1-weighted } \\
\text { images }\end{array}$ & $77.2( \pm 14.9)^{\mathrm{c}}$ & $167.8( \pm 17.2)^{\mathrm{a}}$ & $41.6( \pm 30.2)^{\mathrm{d}}$ & $44.5( \pm 25.1)^{\mathrm{d}}$ \\
\hline $\begin{array}{l}\text { T2-weighted } \\
\text { images }\end{array}$ & $16.1( \pm 2.65)^{\mathrm{d}}$ & $18.7( \pm 1.84)^{\mathrm{d}}$ & $115.1( \pm 17.3)^{\mathrm{b}}$ & $192.7( \pm 25.1)^{\mathrm{a}}$ \\
\hline PBS control & $31.0( \pm 13.0)^{\mathrm{d}}$ & $36.5( \pm 10.4)^{\mathrm{d}}$ & $13.3( \pm 6.24)^{\mathrm{d}}$ & $13.8( \pm 3.93)^{\mathrm{d}}$ \\
\hline FB139 control & $166.0( \pm 3.63)^{\mathrm{a}}$ & $173.2( \pm 2.16)^{\mathrm{a}}$ & $162.0( \pm 3.94)^{\mathrm{a}}$ & $181.7( \pm 3.08)^{\mathrm{a}}$ \\
\hline
\end{tabular}
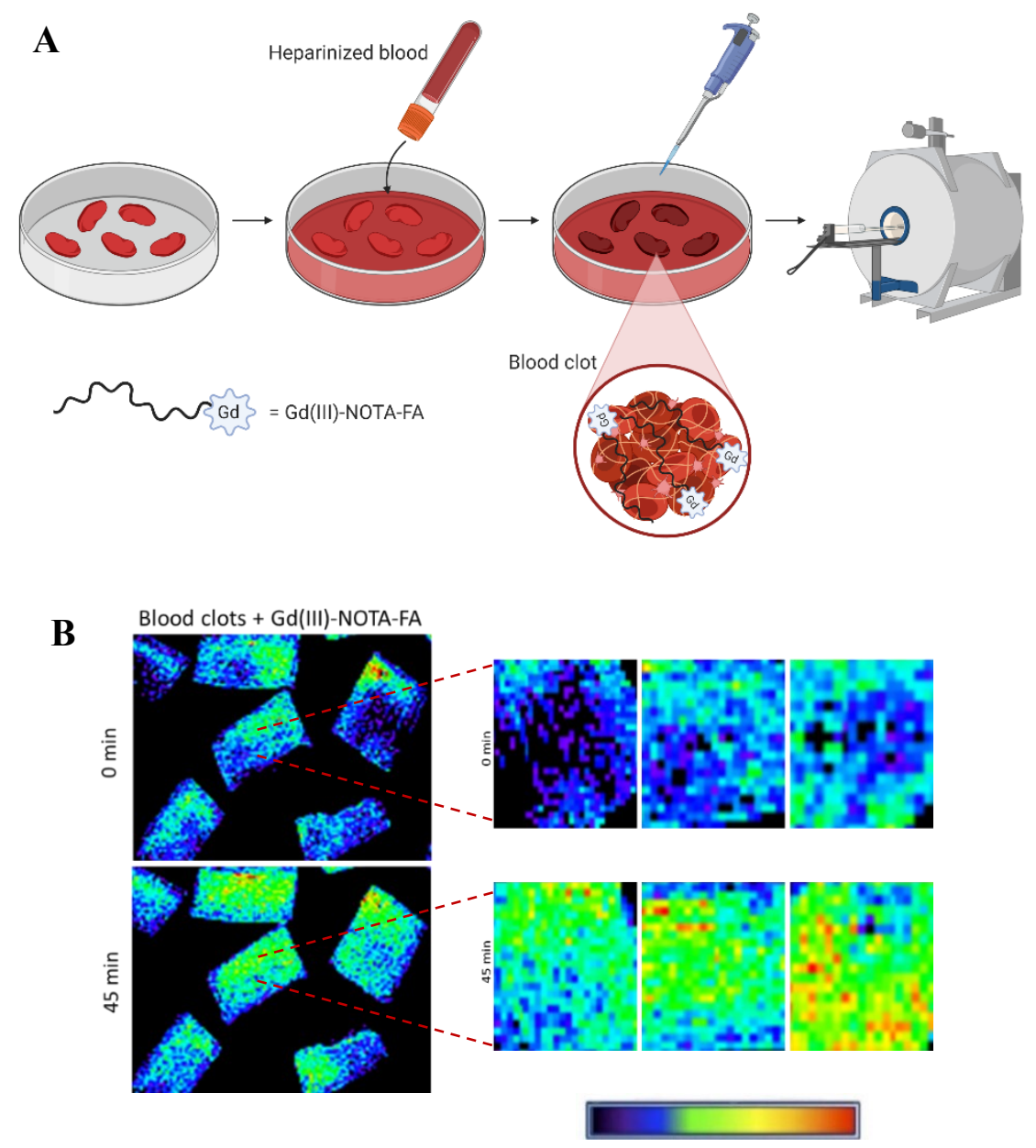

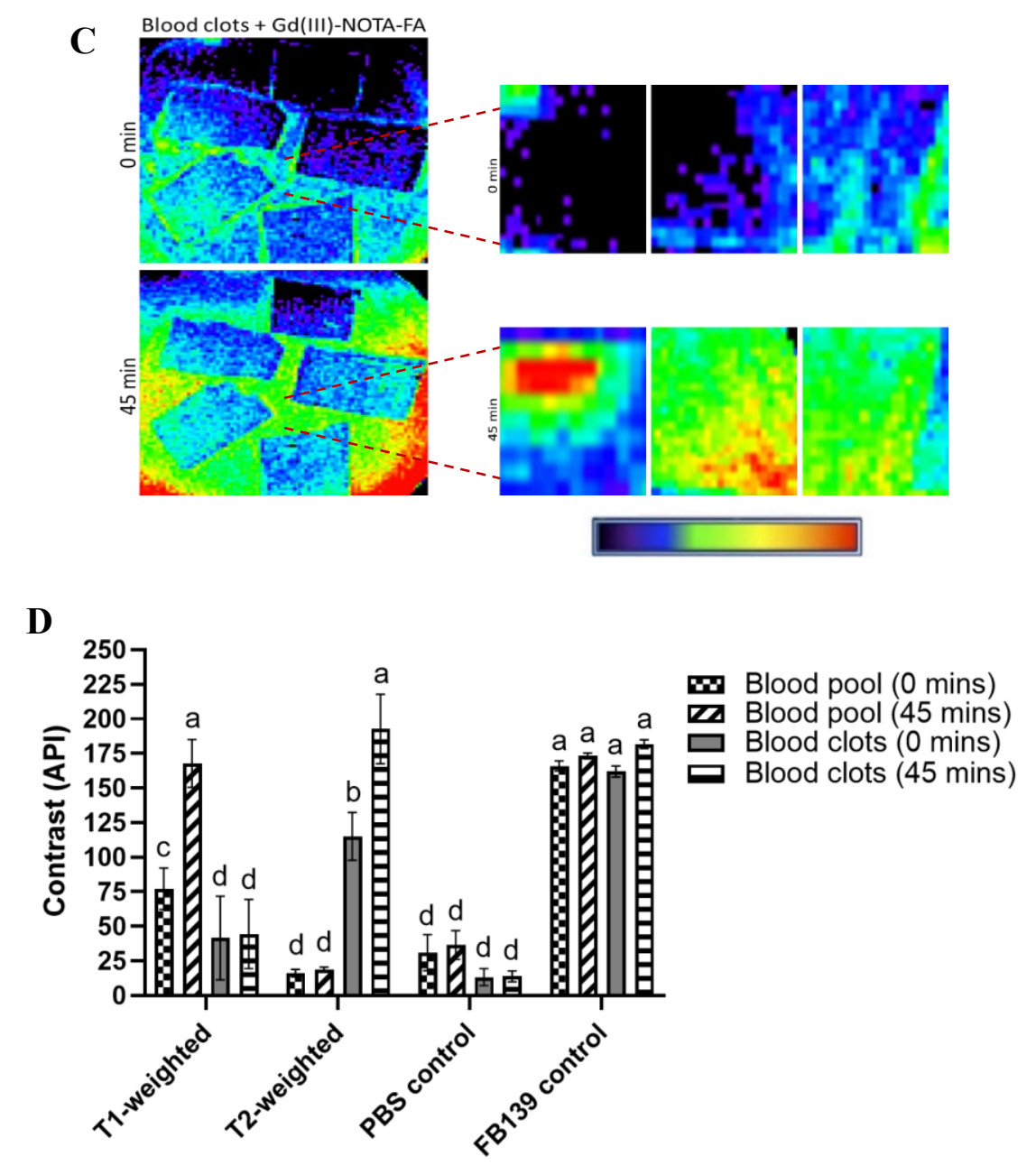

Figure 3.8: (A) Schematic representation of sample preparation for in vitro MRI imaging, made in BioRender; (B) T2-weighted MR images of clotted blood in heparinized blood pool treated with Gd(III)-NOTA-FA in PBS, pH 7.4, demonstrating the development of hyperintense signal at the location of the blood clots (blue to green, with panels representing zoomed images of 3 clots before and after treatment) over a 45-minute scan; (C) T1-weighted MR images of clotted blood in heparinized blood pool, treated with Gd(III)-NOTA-FA in PBS, pH 7.4, demonstrating the development of hyperintense signal throughout the blood pool (blue to green, with panels representing 3 different areas of the blood pool before and after treatment) over a 45-minute scan; (D) Average contrast enhancement of each sample, at 0 mins and 45 mins, measured by ImageJ Software and compared using One-way ANOVA test $(F(15,92)=104.8, p<0.0001)$; Tukey post-hoc analyses revealed a significant difference between treatments $(\mathbf{p}<\mathbf{0 . 0 1})$. Significance is denoted by letters a through $\mathrm{d}$ and represents which treatment comparisons were made. 
To the best of our knowledge, this is the first time that a fibrinogen aptamer-contrast conjugate system has been synthesized and tested in an in vitro system that mimics vascular clotting conditions. We were able to demonstrate that, since the fibrin network is made up of predominantly unaltered monomers of fibrinogen, the aptamer that was selected to bind fibrinogen monomers will continue to bind the polymerized form. ${ }^{26,28}$ In fluorescence microscopy experiments, this binding was visually and statistically confirmed based on the presence of green fluorescence at the fibrin clot. This binding was almost immediate, occurring as quickly as 30 seconds after treatment, and strong enough to withstand several washes with excess buffer. It was overall confirmed that FA is able to bind fibrin in a selective manner. Fluorescence was also used to determine that 48 hours is the optimal time of incubation of the aptamer with the target in preparation of non-solution-based phantom MRI samples. While this result was useful in the optimization of the phantom system, it is does not have clinical relevance, as in a biological system aptamer-target interaction will be based on the speed of blood circulation. ${ }^{172}$ Upon FA's entrance into the blood stream, it will be distributed throughout the circulatory system, with binding events increasing within seconds, while being concentrated at the location of a blood clot.

Measuring longitudinal relaxivity of Gd(III)-NOTA-FA demonstrated that conjugation of the paramagnetic ion-bearing chelator to the long aptamer stand has an adverse effect on $\mathrm{T} 1$ relaxation times. This effect is further propagated in the presence of the fibrous target. It was concluded that steric constraints are likely the most dominant factor affecting longitudinal relaxivity of this system. In order to minimize this effect, aptamer strand truncation would be recommended. This was attempted by cutting the 3'or the 5'-primer binding regions, both primer binding regions, or at nucleobase 65 (Figure 
S3.2A). It was predicted that this may decrease the steric hindrance and allow for more economical production of the final contrast conjugate. Unfortunately, studies assessing affinity and specificity of the binding of the above-mentioned minimers to the fibrin target were inconclusive at this time and require further investigation (Figure S3.2B).

On the other hand, we were able to demonstrate that transverse relaxation rates are enhanced in both the aptamer chelator conjugate, and in the presence of the fibrous target. The shift towards the outer-sphere effects being more dominant suggested that the formation of aptamer-target complex leads to intercalation of the paramagnetic ion. The effects of target binding, and the selectivity of those effects towards fibrin were confirmed through phantom T2 map imaging in the presence of fibrin powder. T2 relaxivity was enhanced in the presence of fibrin, but not in the presence of the control protein, collagen. Importantly, when $\mathrm{Gd}(\mathrm{III})$ chelator was conjugated to an aptamer that is not specific towards either of the protein, no such enhancement was produced, reaffirming specificity of this effect. In a more clinically relevant phantom study, a larger in-lab synthesized fibrin clot exhibited more dramatic contrast enhancement than the fibrin powder. Visualizable dark network of fibers associated with Gd(III)-NOTA-FA interaction with the entire fibrin network suggested that the aptamer conjugate is able to penetrate the surface of the clot. This indicates that FA not only binds to the monomers that are available on the surface of the fibrin clot, as previously postulated, but is also able to differentiate into the polymer network. In addition, we were able to show that, even though FA was originally selected for fibrinogen, it exhibits preferential binding towards fibrin due to receptor clustering. In vitro studies allowed for assessment of this binding in the presence of a complex matrix, using whole blood to mimic vascular environment. Aptamer-targeted delivery of the Gd- 
NOTA contrast agent was observed during both T2- and T1-weighted scans. Remarkably, enhancement of signal intensity was produced exclusively at the location of the clot during the T2-weighted scan, while the presence of fibrinogen within whole blood pool resulted in T1 signal intensity enhancement throughout the pool. This is advantageous as simply reversing the type of a scan from a typical T1-weighted to a T2-weighted would allow to selectively highlight the location of blood clots.

Since this FA conjugate is designed to selectively deliver Gd(III) contrast to fibrin clots resulting in higher contrast enhancement localized at the target, it is expected that in a biological system, the most dramatic contrast enhancement will be localized at the location of blood clots. Because the injected contrast media will be aptamer-guided, smaller concentrations of it will be required based on the target-specific accumulation. This would in turn limit concentration-based side-effects, such as kidney failure. ${ }^{14,53,155,161}$ High resolution between blood clots and the blood pool can be achieved through T2-weighted scans. If these contrast conjugates are used for other purposes and need to cross the blood brain barrier (BBB), they can be used in conjunction with aptamers that have been selected to pass through the BBB. This is accomplished through targeting of the transferrin receptor and could be utilized in a chimeric formulation. ${ }^{199-201}$ Within the scope of this research, aptamer-guided contrast delivery would assist in faster and more specific contrast enhancement. ${ }^{175}$ If FA-conjugate system is successful, this contrast agent may have numerous applications in radiographical imaging and be developed to help the diagnosis of other conditions that involve fibrin formation, including pulmonary embolism and deep vein thrombosis. ${ }^{202}$ 


\subsection{Supporting information}

\subsubsection{Additional methods}

\subsubsection{Assessment of binding kinetics and minimers}

Fluorescence microscopy was used to assess how quickly FA is able to bind fibrin and to determine if this binding is relevant on a clinical scale. As previously described, fibrin clots were synthesized by combining a solution of $20 \mathrm{mg} / \mathrm{mL}$ of fibrinogen, a solution of $0.1 \mathrm{M} \mathrm{CaCl}_{2}$ and $240 \mathrm{IU} / \mathrm{mL}$ of thrombin in phosphate buffered saline (PBS) (pH 7.4). ${ }^{170,171}$ The mixture was incubated for an hour at $37{ }^{\circ} \mathrm{C}$ and the resulting clot was cryosectioned on a Leica CM1900 cryostat to 60 microns. Fibrin slices were subsequently fixed onto a glass slide and $20 \mu \mathrm{L}$ of $5 \mu \mathrm{M}$ 6-FAM FA was applied directly on top. Unbound DNA was washed away with PBS, pH 7.4, subsequent to incubations of 30 seconds, 1, 5, 10 and 30 minutes. All samples were imaged in triplicate with BioRad Fluoro-Micro fluorescence microscope, under blue and green channels, with excitation wavelengths of $355 \mathrm{~nm}$ and $480 \mathrm{~nm}$, respectively. Image analysis was performed using Image J software, measuring average pixel intensities of all samples.

In addition, in order to assess the feasibility of truncating the aptamer strand,

minimers were conceptualized and assessed using MFold software. ${ }^{203}$ The experimental set-up was the same as above and was repeated for each candidate, with FA-m1 cut at the 5'-primer binding region, FA-m2 cut at the 3'-primer binding region, FA-m3 cut at both the 3'- and the 5'-primer binding regions, and FA-m4 cut at nucleobase 65. 


\subsubsection{Phantom MRI optimization}

In order to optimize the phantom system prior to MR imaging, aptamer conjugate movement through the agarose gel was monitored using fluorescence with AlphaImager. Previously prepared fibrin clots were sectioned into uniform portions $(0.5 \mathrm{~cm} \times 0.5 \mathrm{~cm})$, embedded into $0.6 \%$ agarose gel and prepared in triplicate. Aptamer conjugate movement was monitored by directly applying $80 \mu \mathrm{L}$ of $5 \mu \mathrm{M}$ 6-FAM FA in PBS, pH 7.4, to the top of the gel system. Images were acquired prior to adding 6-FAM FA, after a 24-hour and a 48-hour incubation with 6-FAM FA. Unbound aptamer was removed using the concentration gradient principle by incubating all samples with deionized water for 1 week. ${ }^{204}$ Images were acquired using transillumination light with excitation wavelength of $302 \mathrm{~nm}$ at each time point. 


\subsubsection{Additional results and discussion}

\subsubsection{Fluorescence microscopy}
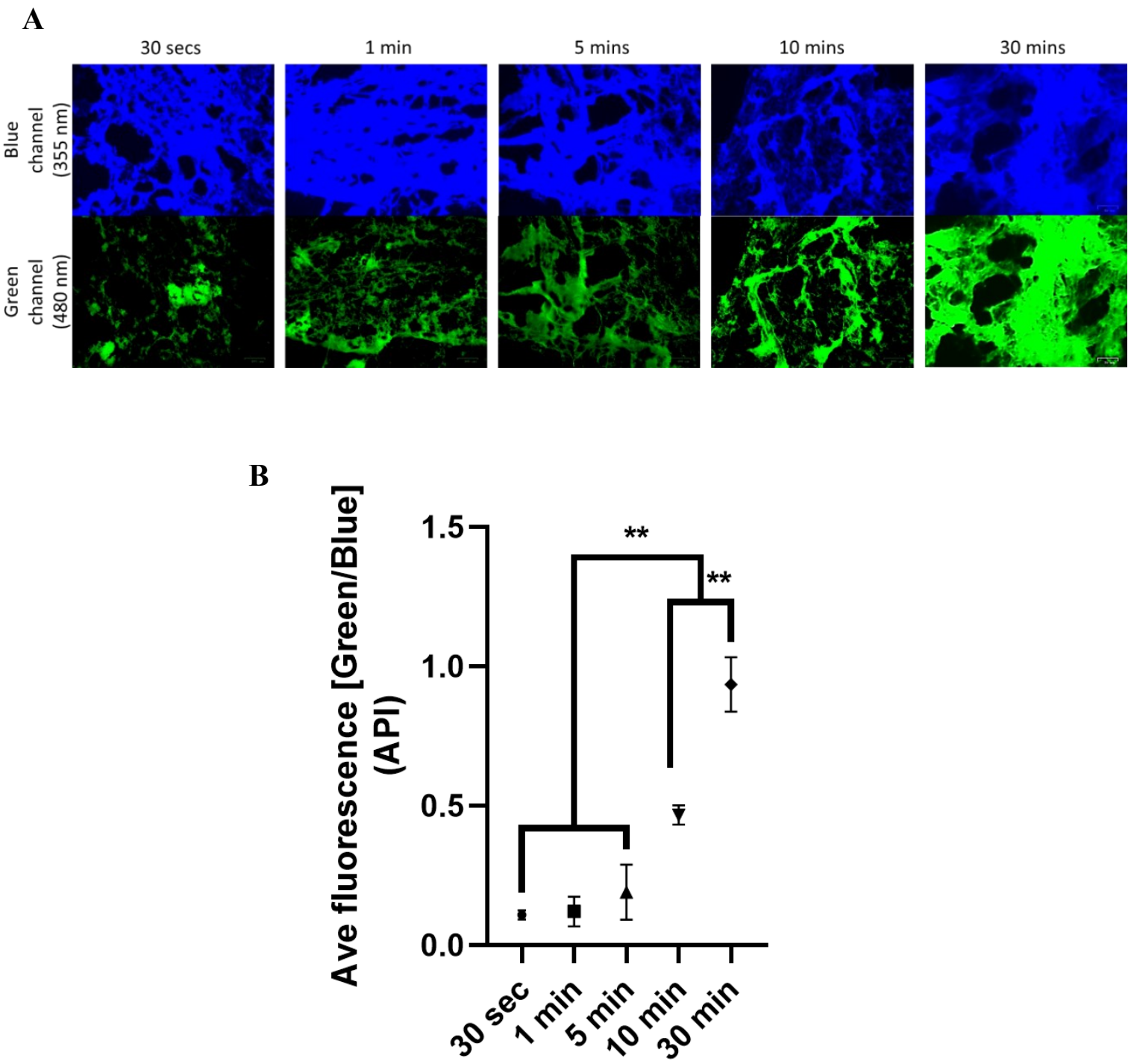

\section{Incubation times}

Figure S3.1: (A) Fluorescence microscopy images of 6-FAM FA incubated with fibrin, under blue and green channels, with excitation wavelengths of $355 \mathrm{~nm}$ and $480 \mathrm{~nm}$, respectively: 30 second, 1 minute, 5 minute, 10 minute incubation, and 30 minute incubations; (B) Average ratios of green to blue fluorescence at different time-points, measured by ImageJ and compared using One-way ANOVA statistical analysis $F(4,23)=148.0, p<0.001)$, Tukey post-hoc analyses revealed a significant difference between groups $* * \mathbf{p}<0.01)$. Significance is indicated by**. 
A

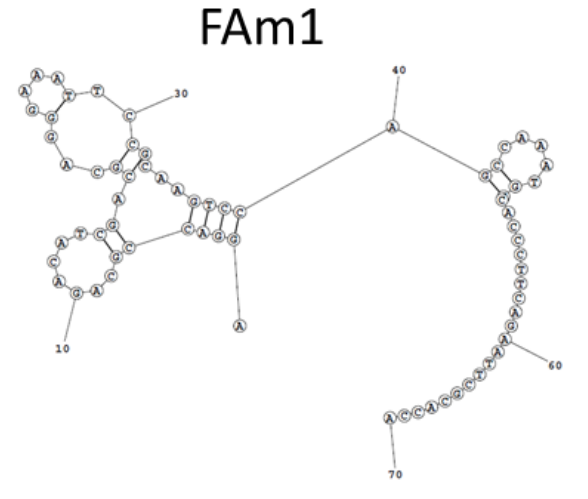

FAm3

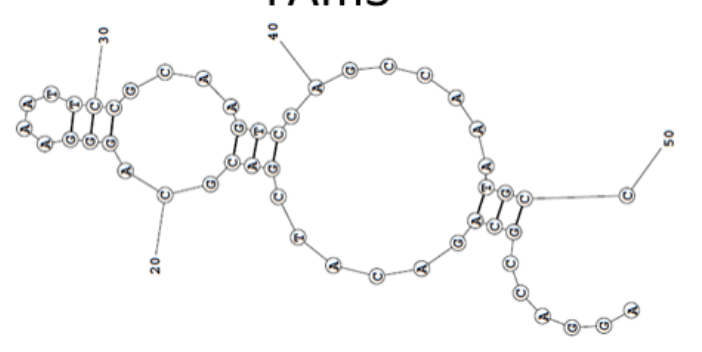

FAm2

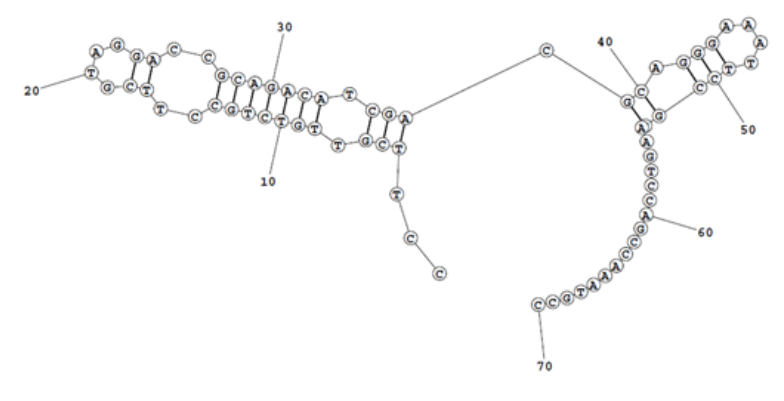

FAm4

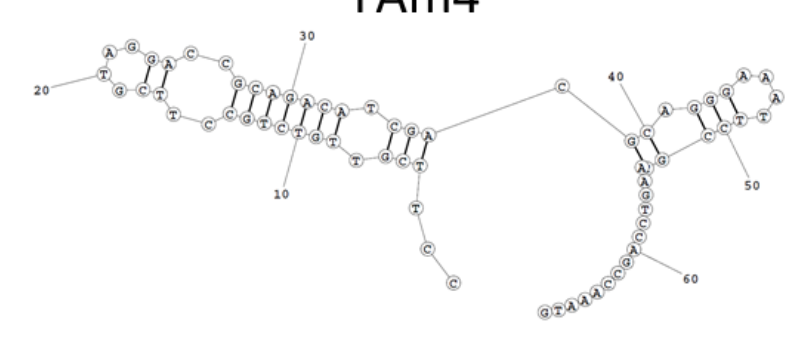

B FA-m1

FA-m2

FA-m3

FA-m4

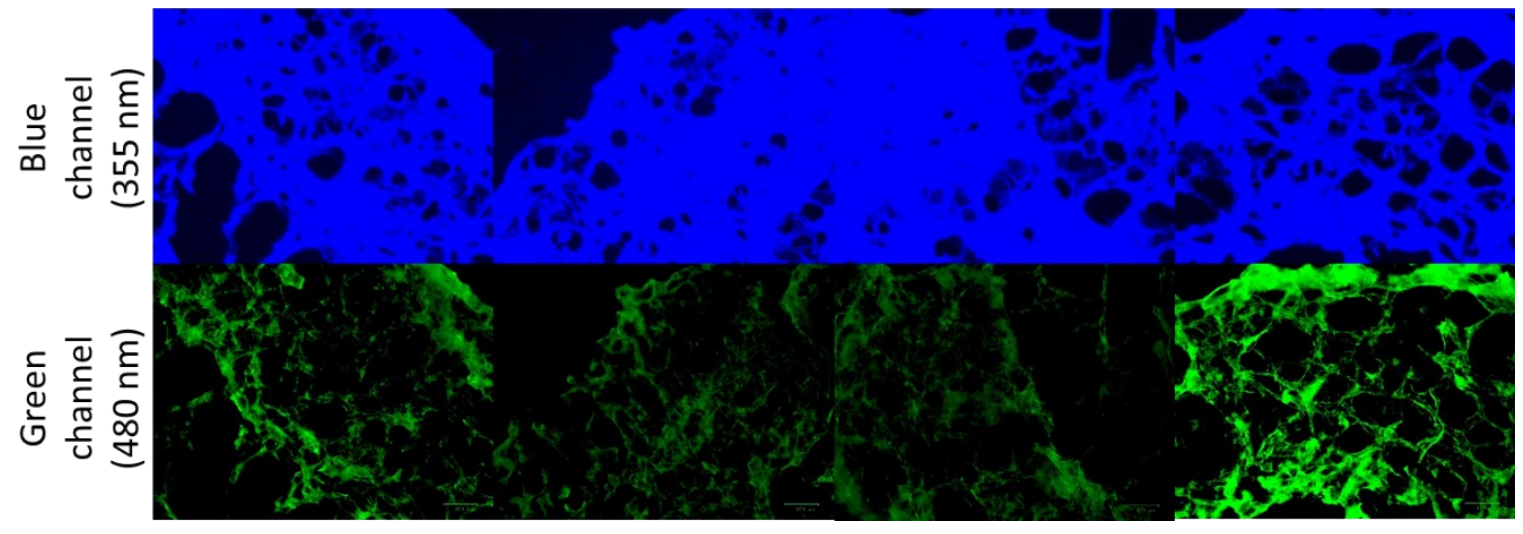

Figure S3.2: (A) Predicted secondary structures of FA-m1, FA-m2, FA-m3, FA-m4 folded by Mfold; (B) Fluorescence microscopy images of 6-FAM FA-m1, 6-FAM FA-m2, 6-FAM FA-m3, 6-FAM FAm4 incubated with fibrin, under blue and green channels, with excitation wavelengths of $355 \mathrm{~nm}$ and $480 \mathrm{~nm}$, respectively. 


\subsubsection{Phantom system fluorescence}

This experiment allowed for optimization of sample preparation and incubation conditions prior to phantom MRI scans. Since the aptamer-target interaction was dependent on diffusion of the aptamer into agarose gel, timescale of these experiments was not expected to reflect that of a biological system MRI scan. ${ }^{147,172}$ Previous to incubation with fluorescently-modified FA (6-FAM FA), fibrin did not demonstrate fluorescence when imaged under white light (Figure S3.2A) or with transillumination light under excitation wavelength of $302 \mathrm{~nm}$ (Figure S3.2B). A 24-hour incubation did not allow for 6-FAM FA to fully diffuse into the agarose gel as seen by fluorescence only reaching approximately half-way into the sample (Figure S3.2C). However, the interaction of FA with the fibrin target was already evident, visualized by high fluorescence co-localization at the top of the fibrin clot (Figure S3.2C). After a 48-hour incubation, which allowed 6-FAM FA to diffuse throughout the agarose gel and interact with the fibrin clot, high fluorescence is achieved throughout the clot (Figure S3.2D), leaving the background (agarose gel), only slightly fluorescent. Notably, fluorescence was particularly high around the edges of the fibrin clot with some fluorescence evident inside the clot. This suggested that FA is able to penetrate into the fibrin clot and interact internally. Removal of unbound 6-FAM FA by equilibrium, utilizing the principle of molecule migration based on the concentration gradient ${ }^{204}$, did not result in a decrease of fluorescence around the fibrin target (Figure S3.2E). High fluorescence was still present around the edges of the fibrin protein, and internal interaction of 6-FAM FA with fibrin remained, demonstrated by fluorescence dispersed throughout the clot. 
To further confirm the visually observed trend, API of each of the time-points was measured by ImageJ software (Figure S3.2F). One-way ANOVA test verified that there was a statistical difference between samples throughout the incubation time-points ( F ( 3 , $8)=592.1, p<0.0001$ at $\alpha=0.01)$. Post-hoc Tukey HSD analysis confirmed the observed trend, and when compared pair-wise, revealed a statistically significant difference between the samples before 6-FAM FA incubation and post 24-hour incubation $(\mathrm{p}<0.001)$, as well as post 24 -hour incubation and post 48 -hour incubation ( $<<0.001$ ), while there was no statistical difference between the samples post 48-hour incubation and after unbound 6FAM FA was removed by equilibrium for a week $(\mathrm{p}=0.90)$. This trend reiterates that FA retains strong binding affinity towards fibrin. 

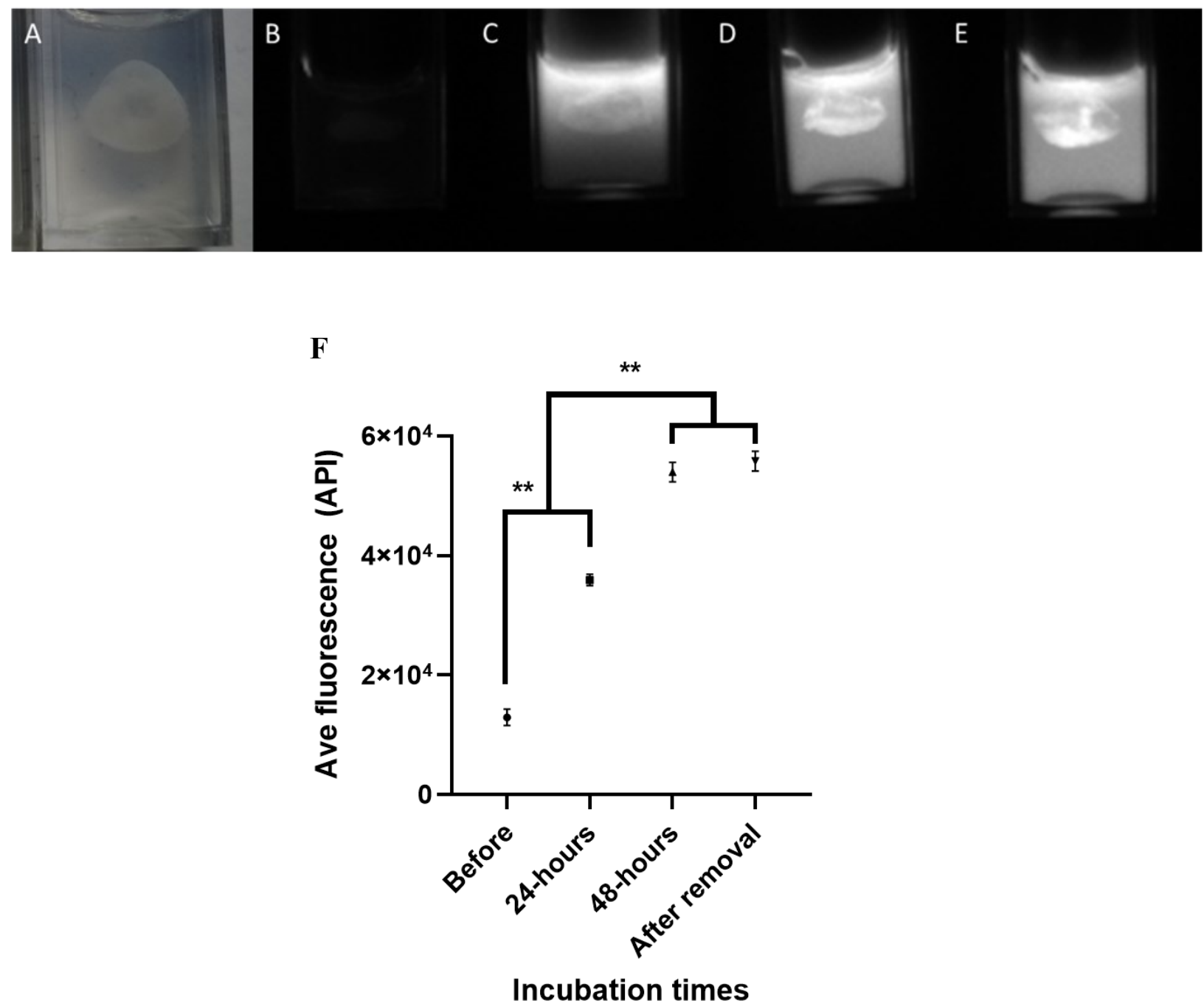

Figure S3.3: Fibrin protein embedded in 0.6\% agarose gel under white light (A); AlphaImager images, under excitation wavelength of $302 \mathrm{~nm}$ : (B) fibrin protein control, (C) fibrin incubated with 6-FAM FA, after 24 hours, (D) fibrin incubated with 6-FAM FA, after 48 hours, (E) fibrin incubated with 6FAM FA, after the gel was covered with $\mathrm{H}_{2} \mathrm{O}$ for 1 week to remove unbound aptamer; (F) Average fluorescence at different time-points of 6-FAM FA incubations, measured by ImageJ and compared using One-way ANOVA statistical analysis $(F(3,8)=592.1, p<0.0001)$, Tukey post-hoc analyses revealed a significant difference between groups $(* * \mathbf{p}<0.01)$. Significance is indicated by **. 


\subsubsection{Gd(III)-NOTA-FA synthesis and assessment}

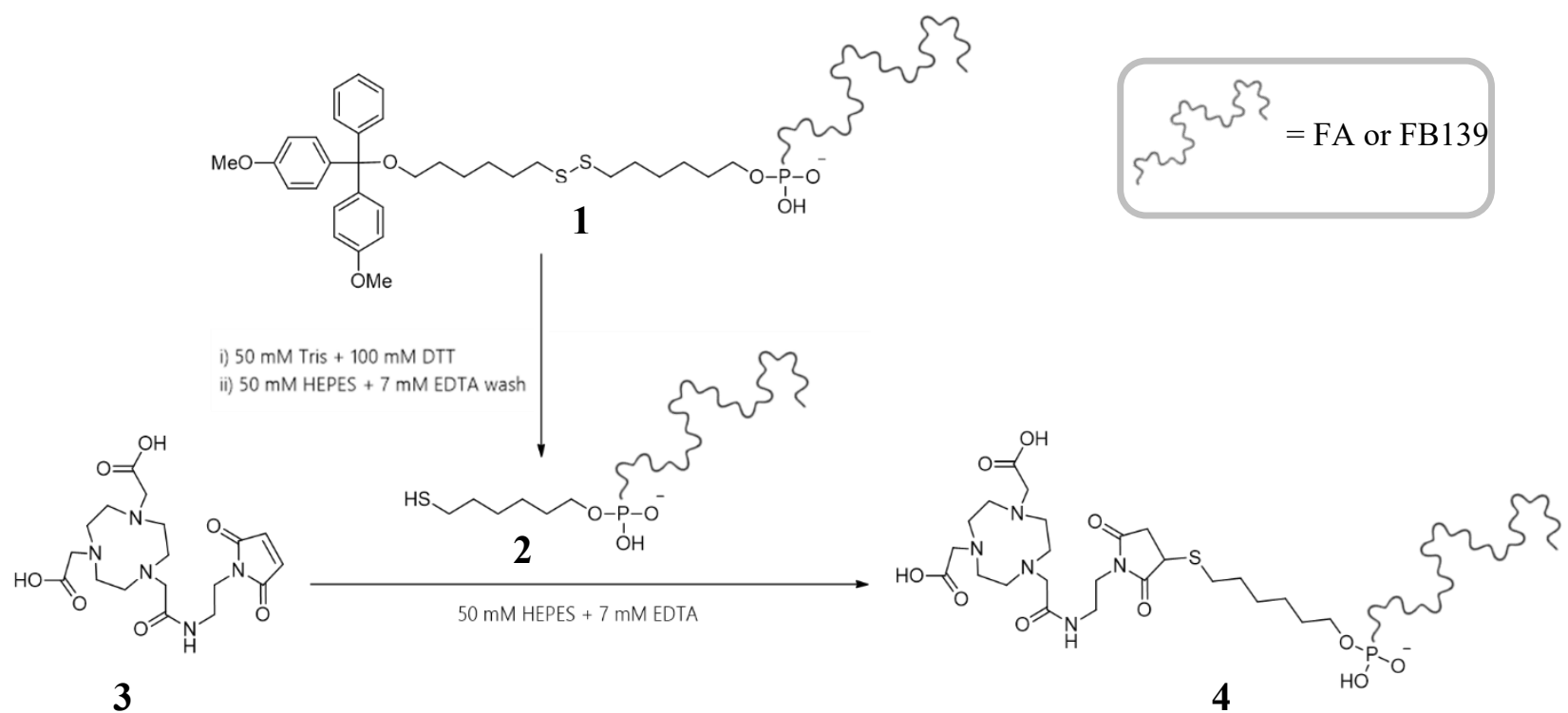

Scheme S3.1: Reaction scheme outlining the cleavage of the thiol-modifier C6 S-S and subsequent conjugation to maleimido-monoamide-NOTA to produce FA-NOTA conjugate. ${ }^{109}$ 


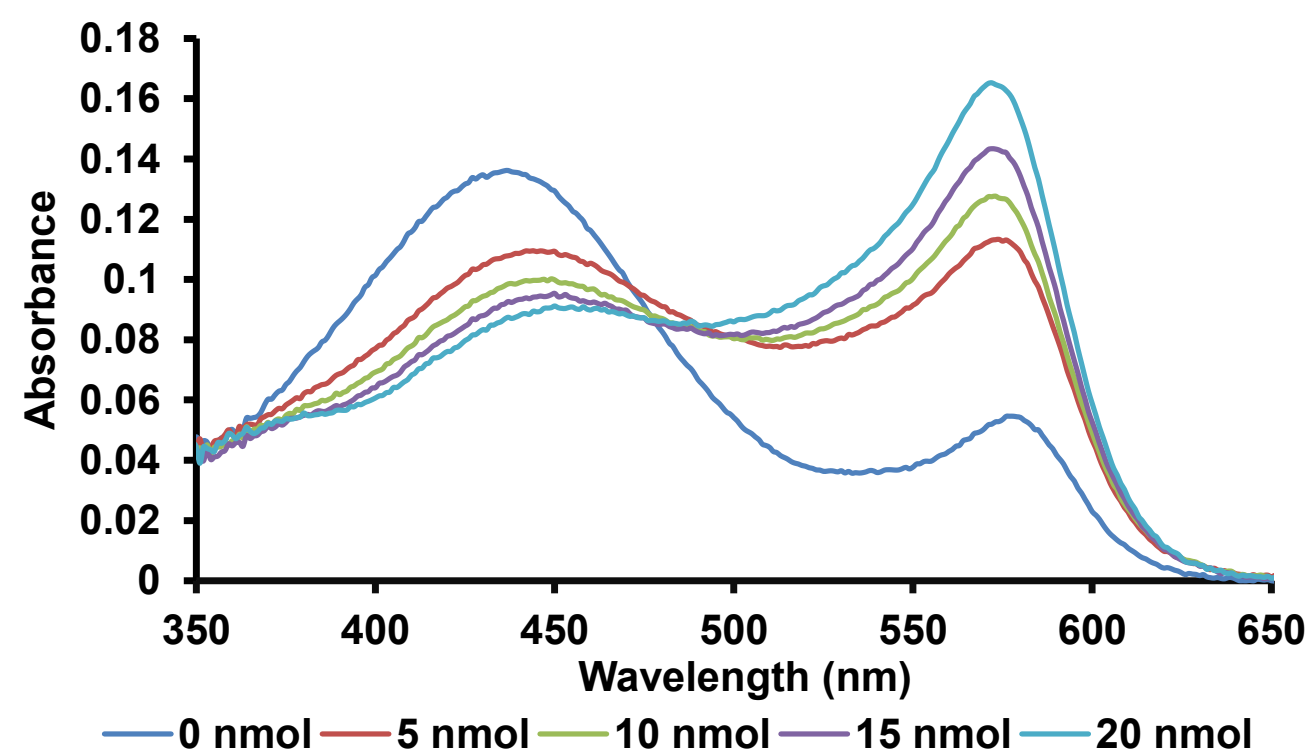

Figure S3.4: Spectrophotomeric determination of $\mathrm{Gd}^{3+}$ complexed by Xylenol Orange. The increase in Gd(III) concentration causes a decrease of the band intensity at $433 \mathrm{~nm}$ and a consequent increase of the band at $573 \mathrm{~nm}$. The spectra were recorded in acetic buffer solution at $\mathrm{pH} 5.8$ in the presence of 0 , $5,10,15,20 \mathrm{nmol}$ of $\mathrm{Gd}^{3+}$ respectively.

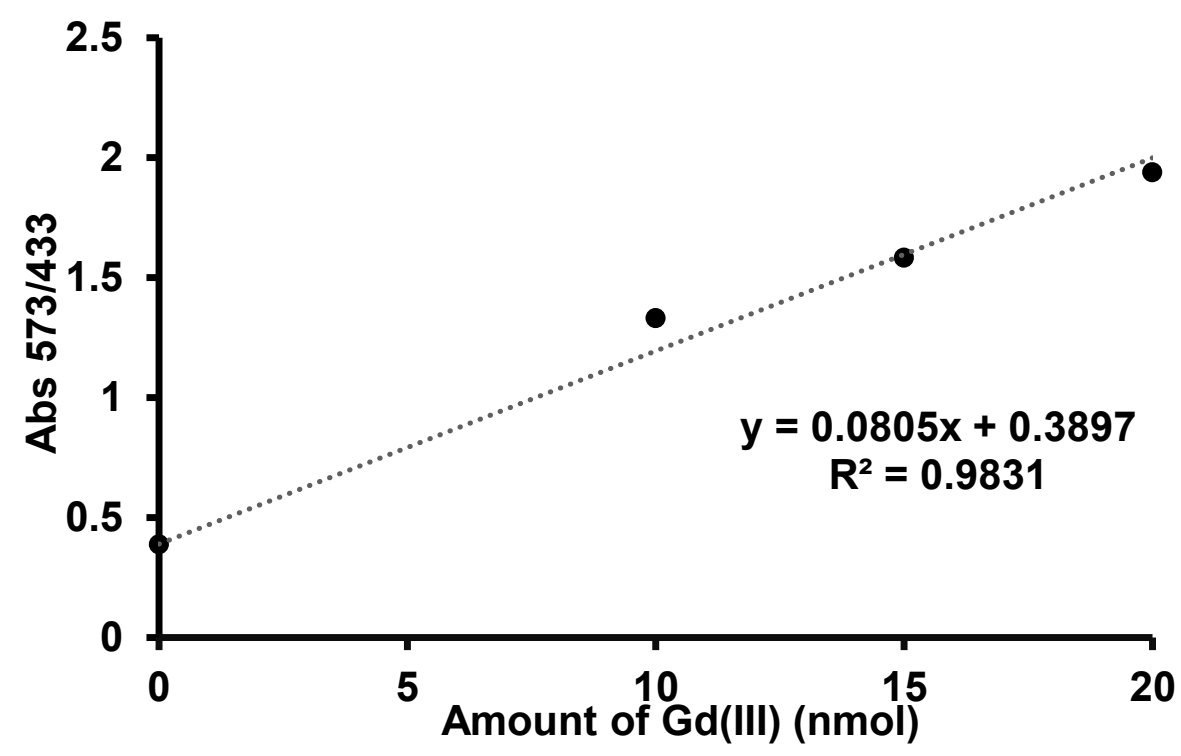

Figure S3.5: Calibration curve obtained by spectrophotometric changes of Xylenol Orange absorptions in the presence of different amounts of $\mathbf{G d}^{3+}$. 
Gadolinium was loaded into each DOTA/NOTA-FA and DOTA-FB139 at 1:1 concentration. Loading efficiency was calculated based on the relationship between these concentrations and the amount of free Gd extracted from the standard curve (Table S3.1).

Table S3.1: Xylenol orange test absorbance values of DOTA and NOTA complexes, with determination of concentration of free $\mathrm{Gd}^{3+}$ and loading efficiency, using $\mathrm{y}=\mathbf{0 . 0 8 0 5 x}+\mathbf{0 . 3 8 9 7}$

\begin{tabular}{|l|l|l|l|}
\hline & NOTA-FA & DOTA-FA & DOTA-FB139 \\
\hline Abs at 573 & 0.060 & 0.056 & 0.055 \\
\hline Abs at 433 & 0.142 & 0.125 & 0.136 \\
\hline Free Gd (573/433) & 0.424 & 0.447 & 0.408 \\
\hline x & 0.412 & 0.695 & 0.218 \\
\hline Total lost (nmol) & 2.412 & 2.695 & 2.218 \\
\hline \% lost (using original concentrations) & 8.7 & 10.2 & 11.1 \\
\hline Loading efficiency (\%) & 91.3 & 89.8 & 88.9 \\
\hline
\end{tabular}

\subsubsection{Clot stability assessment}

It was important to assess if the presence of FA would have any effect on clotting, as fibrinogen is classified as an acute phase protein. ${ }^{26}$ Though the aptamer candidate that was chosen for this work was postulated to bind the regions located away from those that are involved in fibrinogen polymerization into fibrin, any possibility of clotting cascade disruption must be eliminated before translation into in vivo. ${ }^{28}$ Blood was clotted without the aptamer and monitored for 24 hours. Meanwhile, FA was injected directly into the blood and allowed to equilibrate, providing an opportunity for it to interact with fibrinogen present in the blood pool. Clotting was induced in the same manner as with the control clot and was monitored for 24 hours. It did not appear that clot formation was stunted in the presence of FA. Additionally, upon extraction from the container in which the clots were formed, the integrity of the control and the FA-treated clot appeared to be the same (Figure 
S3.6). Cutting both clots did not lead to rips. Overall, injecting FA into the blood before the initiation of clotting cascade did not show any considerable effect, allowing to draw the conclusion that FA indeed binds the regions that are unmodified during hemostasis.

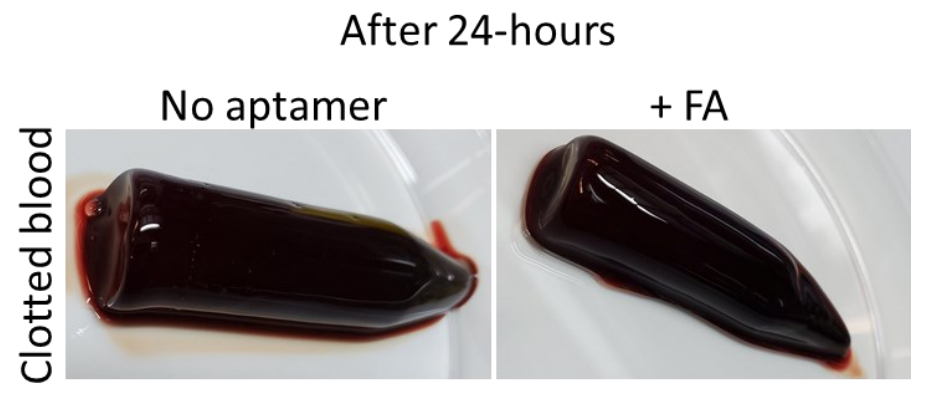

Figure S3.6: Left - human blood after clotting with thrombin, in the presence of $\mathrm{CaCl}_{2}$, for 24 hours; right - human blood with FA that was injected and incubated for 30 mins, then clotted with thrombin, in the presence of $\mathrm{CaCl}_{2}$, for 24 hours. 
Chapter 4: Assessment of aptamer-targeted contrast agents for monitoring of blood clots in computed tomography imaging 


\subsection{Statement of contribution}

Aptamer-functionalized contrast materials and blood samples were prepared by A Koudrina, with assistance from S Boisjoli. MC DeRosa assisted with experimental design. MST analysis was performed by 2bind (Germany). D Marquez assisted in ITC set-up and analysis. All CT scans were done at The Ottawa Hospital by J O’Brian. All fluoroscopy scans were done at Baylor College of Medicine by RL Garcia and PTM Kan. EC Tsai is the clinical collaborator from The Ottawa Hospital.

\subsection{Resulting publications}

This chapter is based on an article entitled "Assessment of aptamer-targeted contrast agents for monitoring of blood clots in computed tomography and fluoroscopy imaging" submitted for publication in Bioconjugate Chemistry (Koudrina A, O’Brien J, Garcia R, Boisjoli S, Kan PTM, Tsai EC, DeRosa MC. ACS Bioconjugate Chemistry. Manuscript ID: bc-2020-005259) 


\subsection{Introduction}

Local hemostasis is activated in response to tissue damage or prothrombotic stimuli and leads to the formation of blood clots to seal the sight of the injury. However, malfunctions, stemming from congenital disorders or a diverse group of clinical conditions, may lead to hypercoagulable states, which are highly undesirable. ${ }^{31,34,35,152,205}$ Pathophysiology of thrombosis is the underlying cause of vascular disease states, including, stroke and thromboembolism. In all cases, it has been suggested that multisite blood clot labelling would allow for definitive diagnosis and more effective followup. ${ }^{152,167,206}$ This can be achieved through targeting the molecular components of blood clots. Specifically, fibrin, one of the main molecular constituents of both venous and arterial blood clots, has been indicated as an ideal candidate for this type of targeting. Since fibrin exists in high concentrations in the aforementioned pathologies, but is absent circulating blood, its targeting could permit highly sensitive and well resolved imaging using a single imaging modality. ${ }^{167,206}$

Computed tomography is a well-established non-invasive imaging technique which provides high resolution three-dimensional visual reconstruction of bodily structures. This technology uses the inherent ability of tissues and organ systems to block ionizing energy, or X-rays, in order to produce cross-sectional images that are further processed into the final images. The mass attenuation is dictated by the density of the tissues along the path of the X-ray. Those that possess higher density $(\rho)$ or high atomic number $(Z)$ will have higher X-ray attenuation than their counterparts. It is important to note the same parameters are taken into account when materials are picked to be effective contrast agents. ${ }^{143,144}$ The ability of matter to attenuate X-rays is defined by Hounsfield Units (HU). Everything is 
normalized to the attenuation of water, with an assigned density value of $0 \mathrm{HU}$, and air, with an assigned density value of $-1000 \mathrm{HU}$. Challenges in obtaining high resolution images arise when scanning soft tissues, which do not display drastically different attenuation values and fall within the range of 30 to $100 \mathrm{HU}$. Some outliers include the lung tissue, with attenuation values of $-1000 \mathrm{HU}$, and mineralized tissue (bone), with attenuation values of $1000 \mathrm{HU} .{ }^{143,144}$ Since the final value of $\mathrm{HU}$ is a reconstruction of Xray attenuation measurements taken around a single axis of rotation, it can to be used to understand the nature and the composition of the tissue being examined. ${ }^{143-145}$

Fluoroscopy is an imaging technique that uses the same principles as CT. One of the notable advantages of using this imaging modality is that it allows for acquisition of real-time, continuous images. With the help of contrast agents injected into blood vessels, fluoroscopic angiography can be used to assess blood flow, or help visualize vasculature. $^{207,208}$ In the context of vascular occlusions, fluoroscopic angiographymonitored mechanical thrombectomy is a rapidly evolving treatment option. ${ }^{152,208}$ Unfortunately, with large vessel occlusions, monitoring the entirety of the arterial anatomy and full delineation of clot pathology becomes difficult. ${ }^{208}$ This is due to this monitoring relying on either anatomic and mechanical vascular abnormalities or deficiency in blood flow, neither of which are directly related to clot composition. ${ }^{206}$

Contrast agents work on the principle of a large increase in absorption coefficient when the incident X-ray energy is equal to or greater than the binding energy of the K-shell electron of an atom. Commonly, iodine and barium are the atoms used as contrast media, as they fall into the category of materials with high atomic number. In order to obtain the highest contrast enhancement, the energy of the X-ray source is adjusted to closely match 
the contrast material's absorption edge value. ${ }^{143,144}$ Small molecules consisting of a central 2,4,6-triiodinated benzene ring are among the most commonly used contrast agents. They possess the most desirable properties like high water solubility, low binding to biological receptors, low toxicity, and high bio-tolerability. Some of the common agents include iohexol (Omnipaque, GE Healthcare), iopromide (Ultravist, Bayer Healthcare), iopamidol (Isovue, Bracco Imaging Canada). Their effective diagnostic dose is in the molar concentration range. ${ }^{143}$ For example, iohexol, which bears the triiodinated benzene core, is able to produce positive contrast enhancement of 25-30 HU per milligram of iodine per milliliter at X-ray energy range of $100-120 \mathrm{kVp} .{ }^{144,147}$ As expected, there is a relationship between contrast enhancement capabilities and iodine concentration within the system, which typically applies to all X-ray energy ranges. ${ }^{147}$ One of the major drawbacks of the commercially available iodinated contrast is the inherently low sensitivity of CT imaging towards the detection of these agents $\left(\sim 10^{-3} \mathrm{M}\right)$, which means that much higher concentrations are required. ${ }^{209}$ With this in mind, molecular targeting can be implemented to achieve high concentrations of reporter elements through preferential accumulation at the chosen target sites. ${ }^{144}$ Aptamers are an example of such ligands, that offer several advantageous characteristics. ${ }^{210}$

Aptamers are short single-stranded oligonucleotides that are selected in an in vitro screening process of systematic evolution of ligands by exponential enrichment (SELEX). They can be selected for a wide range of targets, anywhere from small molecules to whole tissues and cells, and possess high affinity and specificity. Aptamers are analogous to antibodies; however, some notable advantages include their ease of synthesis and modification, superior stability, and ability to function even outside of physiological 
parameters. In fact, the pharmacokinetic profile of aptamers can be tailored through modulation of metabolic stability, rate of distribution and clearance. Meanwhile, several toxicological studies have demonstrated that aptamers do not elicit an immune response with minimal side-effects at therapeutically relevant concentrations. ${ }^{5-7,12,13}$

In order to tap into the benefits of the currently utilized contrast materials, direct incorporation into the targeting elements can be utilized. Chemical modifications of oligonucleotides are commonly used to impart characteristics that allow for translation into clinical use. These include modifications on the terminal end, modifications of the phosphodiester linkage, modifications of the sugar ring and on the bases, which in turn provides resistance to nuclease degradation, improves target binding affinity, and allows for incorporation of modifications applicable for targeted imaging. Most commonly, nitrogenous base modifications play a vital role in increasing robustness of oligonucleotide therapeutics applied towards gene regulation, while terminal end modifications have been used in both therapeutics and diagnostics. ${ }^{24,211}$ Notably, halogenated nucleobase analogs have shown to be useful in X-ray crystallography studies ${ }^{212,213}$ and in single-photon emission computed tomography (SPECT) imaging when radiolabelled with ${ }^{125} \mathrm{I}^{213,214} \mathrm{In}$ both cases, the utility of iodinated nucleobases in X-ray-based imaging is highlighted. ${ }^{213}$

Here, we propose incorporating iodinated cytosine bases into a previously characterized fibrinogen aptamer (FA) for applications in targeted CT or fluoroscopy imaging. ${ }^{215}$ The goal of this work was to achieve selective accumulation of iodinated contrast material at the location of thrombi, allowing for more detailed characterization in a whole-body assessment. In another approach, we hypothesized that we could push the limits of the required injection concentration by utilizing aptamer-functionalized gold 
nanoparticles (AuNPs). Gold nanoparticles have been identified as an excellent alternative to the clinically available contrast agents, as they exhibit X-ray attenuation higher than that of the iodine-based materials, given the appropriate ionizing energy. Other advantages include ease of surface-modification and low toxicity. ${ }^{75} \mathrm{We}$ proposed that fibrin-targeting capabilities, achieved through incorporation of FA, would allow for optimization of the contrast distribution, thereby providing higher contrast enhancement at considerably lower concentrations. Both, iodinated FA and FA-functionalized AuNPs were tested in vitro for their ability to produce measurable contrast enhancement through selective accumulation at the location of the blood clot, thereby providing high resolution between fluid blood and the clot. Successful formulation was tested in vivo and ex vivo, using a swine model, with a large vascular occlusion serving as an ischemic stroke model.

\subsection{Materials and methods}

\subsubsection{Chemicals and instruments}

DNA aptamers were synthesized on a BioAutomation MerMade6 oligonucleotide synthesizer (Plano, TX, USA), using standard phosphoramidite chemistry. Phosphoramidites, including 5-I-dC-CE Phosphoramidite, 5'-thiol C6 S-S modifier, activator, deblock, capping, and oxidizing reagents were obtained from Glen Research (Sterling, VA, USA). Ultra-High Purity 5.0 argon was purchased from Praxair Canada (Mississauga, ON, Canada). Standard support columns and acetonitrile were purchased from BioAutomation (Plano, TX, USA). All DNA was purified using Glen-Pak cartridges purchased from Glen Research (Sterling, VA, USA) using the appropriate protocol for the synthesized aptamer. Aptamers were quantified by monitoring absorbance at $260 \mathrm{~nm}$ using a Varian (Palo Alto, CA, USA) Cary 300 Bio UV-visible spectrophotometer (Varian, Palo 
Alto, CA, USA). All purified DNA was sent to the Mass Spectrometry center at McGill University (Montreal, QC, CA) in order to obtain molecular weight confirmation of the products. De-salting and separation after the cleavage of the disulfide bond was done using Amicon-Ultra $0.5 \mathrm{~mL} 3 \mathrm{kDa}$ cut-off ultra-centrifugal units purchased from Fisher Scientific Canada (Ottawa, ON, Canada). Deionized water was collected using Millipore (Billerica, MA, USA) Milli-Q water system and used to prepare all buffers. A Cary-17 Spectrophotometer Conversion (Olis Inc., USA) was used to acquire all circular dichroism data. CSC INC 4500 Isothermal Nanocalorimeter was used for isothermal titration calorimetry. Dissociation constant analysis was performed using Prism GraphPad 8.4.3.

Thrombin solution was purchased from Haematologic Technologies Inc. (Essex Junction, VT, USA). Other buffering agents were purchased from BioShop (Burlington, ON, CAN). Dried polyvinylpyrrolidone (PVP)-stabilized gold nanospheres $(15 \mathrm{~nm})$ were purchased from Nanocomposix (San Diego, CA, USA). Blood was obtained from Canadian Blood Services with approval from the Canadian Blood Services Research Ethics Board (application \# REB2018.053). All CT Imaging was done using General Electric HD 750 Discovery CT Scanner (The University of Ottawa) with dual energy protocols at 80/140 kVp. All cerebrovascular studies were performed at the large animal angiography laboratory at Baylor College of Medicine. All procedures were performed in accordance with the protocol approved by the College's Institutional Animal Care and Use Committee (protocol \# AN7957). Fluoroscopy imaging was done Siemens Healthcare GmbH AXIOM-Artis VC21C system. 
Table 4.1: DNA aptamer sequences, their modifications, and associated abbreviations. Iodinated cytosine bases are denoted with *

\begin{tabular}{|c|c|c|}
\hline $\begin{array}{l}\text { Aptamer } \\
\text { name }\end{array}$ & Sequence (5'-3') & Ref \\
\hline Iodo-FA & $\begin{array}{l}\text { CCTTCGTTGTCTGCCTTCGTAGGACCGCAGACATCGACG } \\
\text { CAGGGAAATTCCGCAAGTC*C*AGC*C*AAATGC*C*AC* } \\
\text { C*C*TTC*AGAATTC*GC*AC*C*A }\end{array}$ & 168 \\
\hline $\begin{array}{l}\text { Iodo- } \\
\text { FB139 }\end{array}$ & $\begin{array}{l}\text { ATACCAGCTTATTCAATTAATCGCATTACCTTATAC*C*A } \\
\text { GC*TTATTC*AATTAC*GTC*TGC*AC*ATAC*C*AGC*TTA } \\
\text { TTC*AATTAGATAGTAAGTGC*AATC*T }\end{array}$ & 169 \\
\hline Thiol-FA & $\begin{array}{l}\text { /5ThioMC6-D/ } \\
\text { CCTTCGTTGTCTGCCTTCGTAGGACCGCAGACATCGACG } \\
\text { CAGGGAAATTCCGCAAGTCCAGCCAAATGCCACCCTTCA } \\
\text { GAATTCGCACCA }\end{array}$ & 168 \\
\hline $\begin{array}{l}\text { Thiol- } \\
\text { FB139 }\end{array}$ & $\begin{array}{l}\text { /5ThioMC6- } \\
\text { D/ATACCAGCTTATTCAATTAATCGCATTACCTTATACCA } \\
\text { GCTTATTCAATTACGTCTGCACATACCAGCTTATTCAATT } \\
\text { AGATAGTAAGTGCAATCT }\end{array}$ & 169 \\
\hline
\end{tabular}

\subsubsection{Preparation of aptamer-conjugated contrast materials}

Iodinated FA (iodo-FA): Both iodo-FA and iodo-FB139 were synthesized by directly incorporating 5-I-dC-CE Phosphoramidite (Figure 4.1) into the positions highlighted in Table 4.1 during on-column synthesis, using standard phosphoramidite chemistry. Following the synthesis, mild deprotection protocols were utilized as to preserve the modifications. Iodinated aptamers were dried down and stored in $-80^{\circ} \mathrm{C}$ until use. 


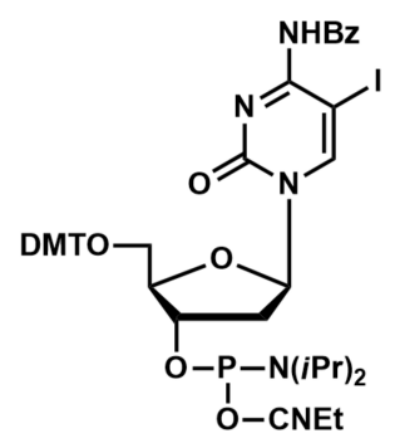

Figure 4.1: Structure of 5-I-dC-CE Phosphoramidite, used in the synthesis of Iodo-FA and -FB139. Structure drawn in ChemDraw.

FA-functionalized AuNPs (FA-AuNPs): Dry PVP-stabilized AuNPs were re-suspended in the appropriate volume of $\mathrm{diH}_{2} \mathrm{O}$ to achieve $1 \mu \mathrm{M}$ concentration, which was confirmed by monitoring the peak at $520 \mathrm{~nm}$ with UV-Vis. FA/FB139 surface-conjugation was accomplished utilizing the Au-S bond. ${ }^{216}$ Thiol-FA/FB139 (60 nmol, Table 4.1) was vortexed in $75 \mu \mathrm{L}$ of $50 \mathrm{mM}$ Tris (pH 8.4) containing $100 \mathrm{mM}$ dithiothreitol (DTT) at ambient temperature for 30 minutes in order to cleave the 5'-disulfide in preparation for conjugation with AuNPs. Cleaved FA/FB139 was washed using BioRad Micro Bio-Spin Chromatography columns, using EDTA buffer (7 mM, pH 7.0) and subsequently de-salted using Amicon Ultra 0.5 mL Centrifugal Filters with HEPES buffer (50 mM, 7 mM EDTA, $\mathrm{pH}$ 7.0). An aliquot of the AuNP solution $(100 \mu \mathrm{L})$ was then combined with $100 \mu \mathrm{L}$ of thiolated (cleaved, $100 \mu \mathrm{M}$ ) FA/FB139. This was shaken overnight to allow for surfacefunctionalization. 


\subsubsection{Aptamer characterization}

\subsubsection{Circular dichroism}

Fibrin was solubilized by dissolving $50 \mathrm{mg}$ of lyophilized fibrin powder in $2 \mathrm{~mL}$ (25 $\mathrm{mg} / \mathrm{mL}$ ) of $1 \mathrm{M} \mathrm{NaOH}$. The final concentration was determined with UV-Vis, monitoring the peak at $280 \mathrm{~nm}$. This was used as the stock solution for all measurements with solution-based protein target. To monitor the structural changes and binding properties of FA to fibrin, an optical chamber of $160 \mu \mathrm{L}$ was used, serving as the final volume of all samples. The CD spectra were acquired in the range of $200 \mathrm{~nm}$ to $400 \mathrm{~nm}$, scanning at $25 \mathrm{~nm} / \mathrm{min}$ speed, with bandwidth of $1 \mathrm{~nm}$, response of $1 \mathrm{~s}$, data interval of 1 $\mathrm{nm}$ and a total number of acquisitions at three. Buffer contributions were baselined at the beginning of all data acquisition. The amount of FA was kept constant, 3 nmol (18 $\mu \mathrm{M}$ in $160 \mu \mathrm{L}$ ), while the concentration of fibrin was incrementally increased, making up the total volume to $160 \mu \mathrm{L}$ with $\mathrm{PBS}, \mathrm{pH}$ 7.4. Each mixture was incubated at room temperature for 30 mins prior to measurement.

\subsubsection{Isothermal titration calorimetry}

Binding buffer (300 mM NaCl, 5 mM MgCl 2,20 mM Tris-HCl, $\mathrm{pH}$ 7.6) was used as the reference to subtract baseline contributions. Each aptamer, unmodified FA and iodoFA were equilibrated at the concentration of $10 \mu \mathrm{M}$ in the sample cell overnight. Fibrin stock was prepared to $200 \mu \mathrm{M}$ concentration at a total volume of $250 \mu \mathrm{L}$. This stock was injected into the sample cell containing the aptamer at $10 \mu \mathrm{L}$ each injection, with a wait interval of $450 \mathrm{~s}$ between each injection, for a total of 20 injections. The temperature was kept constant at $25^{\circ} \mathrm{C}$ and the sample cell was stirred. Areas under the curve for enthalpy 
change $(\Delta \mathrm{H})$ were plotted against fibrin concentration and dissociation constant was calculated using equation 2.3 with BindWorks software.

\subsection{4 vitro CT scans (human blood)}

Whole human blood was clotted using a previously established thrombectomy protocol. ${ }^{152}$ In brief, $1 \mathrm{~mL}$ of $\mathrm{CaCl}_{2}$-thrombin solution was prepared using $0.252 \mathrm{M} \mathrm{CaCl}_{2}$ and $100 \mathrm{U}$ of thrombin. This solution was subsequently mixed with $5 \mathrm{~mL}$ of whole blood and left to form a clot at room temperature for 30 minutes. The large clots were sectioned into approximately $1 \mathrm{~cm}$ in diameter portions and samples were set up in triplicate with 1 $\mathrm{mL}$ pools to mimic a blood vessel environment.

Iodo-FA: Both iodo-FA and iodo-FB139 (control) were re-suspended in PBS, pH 7.4 to 1 mM stock concentration. Samples were incubated $100 \mu \mathrm{L}$ iodo-FA/iodo-FB139, applying the iodinated aptamer directly on top, and allowing it to equilibrate with the target for 30 mins at room temperature. Iodo-FA was used in incubations with blood clots in a heparinized blood pool, and with blood clots in PBS to compare contrast development in the presence and absence of a complex matrix, respectively.

FA-AuNPs: FA-AuNPs and FB139-AuNPs were used directly after synthesis and quantification. Blood clot samples were treated with $100 \mu \mathrm{L}$ of $1 \mu \mathrm{M}$ FA-AuNPs/FB139AuNPs and incubated for 30 mins at room temperature. As previously, FA-AuNPs were used in incubations with blood clots in a heparinized blood pool, and with blood clots in PBS.

Aptamer-treated samples were compared to those that were incubated with $100 \mu \mathrm{L}$ of PBS, or iopamidol (clinical CT contrast). Iopamidol was diluted to $1 \mathrm{mM}$ concentration 
in order to match the concentration of iodo-FA. Since iopamidol is clinically injected in 1 M concentration, further dilutions were not prepared.

\subsubsection{Fluoroscopy scans}

\subsubsection{Clot preparation (swine blood)}

Similar to above, $15 \mathrm{~mL}$ of autologous whole blood was collected from the anesthetized pig via a femoral sheath. Thrombin solution was created by dissolving $7 \mathrm{~g}$ of $\mathrm{CaCl}_{2}$ in $250 \mathrm{~mL}$ of PBS, pH 7.4, $3.3 \mathrm{~mL}$ of which was then mixed with $100 \mathrm{U}$ of thrombin. This thrombin solution was then mixed at a 1:5 ratio with the autologous blood. The blood and thrombin mixture was quickly injected into silicone tubing, prior to initiation of clotting. The clotted material was matured in the tube for 1 hour prior to use. The clot was cut to the desired length (between 15 and $20 \mathrm{~mm}$ ) for creation of the vascular occlusion. This technique was modified from that used by Gounis et al.. ${ }^{152,217}$

\subsubsection{Animal preparation}

All animal procedures were reproduced from a protocol described by Srinivasan $e t$ al.. ${ }^{152}$ Female Yorkshire swine, approximately 7 months old and weighing $60-70 \mathrm{lbs}$, was used. Atropine $(0.12 \mathrm{mg} / \mathrm{kg})$ was administered as an intramuscular preanesthetic agent. Anesthesia was induced by the veterinary care team, using a mix of Telazol $(4.4 \mathrm{mg} / \mathrm{kg})$ and xylazine $(2.5 \mathrm{mg} / \mathrm{kg})$. Mechanical ventilation was given containing oxygen mixed with isofluorane (1\%-3\%). Vital signs were monitored throughout the procedure.

A 9-Fr Femoral Access sheath was placed in the right femoral artery by using a modified Seldinger technique following a cutdown. The short sheath was then secured in place. A 7-Fr Cook Shuttle Guiding catheter (Cook Medical) was advanced over an 0.035- 
in Glidewire (Terumo IS) under fluoroscopy. In order to prevent spontaneous coagulation, blood thinner, Heparin (50 IU/kg), was delivered intravenously. Intraarterial verapamil (10 mg) was infused continuously through the guiding catheter to avoid vasospasm. Under roadmap guidance, the guiding catheter was placed in the common carotid artery (CCA) and a triaxial system was established with a Synchro Standard microwire (Stryker), Trevo 18 microcatheter (Stryker Neurovascular), and ACE68 reperfusion catheter.

After the ACE68 catheter was positioned in the desired location, the clot was backloaded into the device through the Penumbra suction tubing (ID 0.110 in), and then flushed anterograde through the system. Care was taken throughout this procedure to minimize any damage to the clot, and any clots that underwent fragmentation prior to loading into the ACE catheter were discarded so as to use the best possible intact clot for injection. Diagnostic angiography was performed to confirm the occlusion.

\subsubsection{Aptamer preparation}

Iodo-FA was diluted to the concentration of $535 \mu \mathrm{M}$ in PBS. For in vitro imaging, iodo-FA and swine blood clot were first imaged separately. Then, swine blood clot was incubated with $2 \mathrm{~mL}$ of iodo-FA and left overnight. Fluoroscopy scans were performed under a standard protocol. For ex vivo imaging, $4 \mathrm{~mL}$ of iodo-FA was first injected into the face of the clot in vivo over 30 mins. The tissue was subsequently harvested and fixed in formaldehyde overnight before fluoroscopy scans were performed. ${ }^{218}$ 


\subsection{Results and discussion}

\subsubsection{Preparation of aptamer-guided CT contrast materials}

\subsubsection{Iodo-FA}

Aptamers have been subjected to a plethora of modifications to gain specific characteristics; when narrowing the scope to modifications on the bases, the most modified positions are C5- and N7- of pyrimidines and purines, respectively. These modifications have been shown to increase chemical diversity of aptamers and are well tolerated, providing increased stability while ensuring their binding affinity is uncompromised. ${ }^{219}$ FA is an aptamer that shows good binding affinity towards human fibrinogen (64 nM), which is an essential component of the cascade leading towards the formation of fibrin. ${ }^{5-}$ ${ }^{7,28}$ Our group previously investigated the ability of FA to bind non-solution-based fibrin polymer, showing that it retains binding affinity and is able to penetrate the interior of the fibrin network, even in the presence of a complex matrix. ${ }^{215} \mathrm{FA}$ is, therefore, an excellent candidate for proof-of-concept studies in the development of aptamer-targeted contrast media for monitoring of vascular conditions that involve blood clots, using CT imaging. The rational design of the iodine-modified aptamer was centered around a predicted secondary structure folded using MFold, and was conceptualized by our group (Figure S4.1). ${ }^{203}$ Based on the thermodynamically-favored structure, it was postulated that the stem-loops located at the 5' end of FA may to be involved in target binding. Though it is important to remember that this may not be consistent with the structure responsible for binding, it was consistent with the folding and base pairing study conducted by Hamilton et al.. ${ }^{168}$ In order for the contrast enhancement not to be obstructed by the formation of the aptamer-target complex, the iodinated cytosine bases were incorporated into the 3 ' end of 
the aptamer structure, which resulted in total of 14 5-iodocytosines (Figure S4.1). This is nearly 5-times the iodine content of the clinically used contrast agent, iopamidol. An important consideration behind this design was achieving a significant contrast enhancement at low injection concentrations. ${ }^{144,147}$ The 5-iodocytosine bases were incorporated into FA directly during solid-support synthesis, followed by mild deprotection protocols to preserve the modifications. Fully modified FA showed the expected molecular ion peak of 29286.2 Da in mass spectrometry analysis and demonstrated high fidelity from batch-to-batch. The control aptamer, toxin-binding FB139, was chosen based on its similarities to FA in length and base content, and was modified with the same number of iodinated cytosine bases at the 3' end (Figure S4.1). ${ }^{169}$ The expected molecular ion peak of 30119.0 Da was confirmed through mass spectrometry analysis.

\subsubsection{FA-AuNPs}

An example of fibrin-targeting AuNPs was recently reported by Kim et al., who developed glycol-chitosan-coated AuNPs which were conjugated to fibrin-targeting peptides. They were able to demonstrate that the presence of the fibrin-targeting element was absolutely crucial for achieving highly resolved images of thrombi in $\mathrm{CT}{ }^{220} \mathrm{We}$ proposed that FA can also act as a fibrin-seeking moiety, with AuNPs serving as an excellent alternative to the clinically used contrast materials. For the purposes of this research, dry PVP-stabilized AuNPs were used as they can be re-suspended in a predetermined volume to achieve an appropriate concentration for attainment of observable contrast in a CT scan. Thiol modification provided an opportunity for an easy functionalization of AuNPs with FA, which is a surface-conjugation method that is

commonly utilized (Scheme S4.1). ${ }^{216}$ While alternative methods of AuNP surface- 
functionalization exist, including adsorption, incorporation of the aptamer using the goldsulfur bond ensures that AuNPs are delivered to the target upon ligand binding. ${ }^{221}$ The final concentration was determined to be $1 \mu \mathrm{M}$ based on the AuNPs and $450 \mu \mathrm{M}$ based on the DNA in UV-Vis.

\subsubsection{Aptamer binding assessment}

Since this research in part revolved around augmentation of the aptamer bases, several binding validation studies were conducted. To streamline this assessment, lyophilized fibrin from human plasma was solubilized. To ensure that solubilization did not disrupt the overall stability of the target, fibrin concentration was monitored with UVVis, with no quantifiable change after 3 months. (Figure S4.2). ${ }^{30}$

\subsubsection{Circular Dichroism}

To better understand the binding of FA to fibrin, induced structural changes of the aptamer were monitored using circular dichroism (CD). Both aptamers used in this assessment, FA and FB139, contained absorption maxima at approximately $270 \mathrm{~nm}$ and absorption minima at approximately $245 \mathrm{~nm}$, and were confirmed to adopt the B-DNA form. Unmodified FA was tested with a range of concentrations of solubilized fibrin, while FB139 was used as a control, monitoring the peak at $245 \mathrm{~nm}$ for both. Notably, there was a decrease in ellipticity over the range of protein concentrations at approximately $210 \mathrm{~nm}$ (data not shown). However, this is a region where protein ellipticity would have a large contribution (discussed in section 2.2.2) and observed structural changes may simply stem from the net effect of the individual components. With increasing target concentration, an increase in ellipticity at $245 \mathrm{~nm}$ was observed when the fibrin target was incubated with FA (Figure 4.2). In order to predict an apparent dissociation constant associated with the 
binding of FA to fibrin, these changes in ellipticity were plotted against the concentration of the target. The apparent $\mathrm{K}_{\mathrm{d}}$ was calculated to be $5.3 \mu \mathrm{M}$, using the linear dynamic range with a complete one-site saturation model (Table S4.1). Importantly, the changes of ellipticity at $245 \mathrm{~nm}$ when the target was incubated with FB139 were minor (Figure S4.3). This method, unfortunately, indicates a large loss in binding affinity towards fibrin for a number of reasons. In the determination of $\mathrm{K}_{\mathrm{d}}$, it is important to reach a binding plateau at both the lowest and the highest concentrations of the titrated ligand, indicating a range of concentrations where minimal binding occurs and where saturation is reached, respectively. The point of inflection that exists in between is used to calculate $\mathrm{K}_{\mathrm{d} .}{ }^{102,222}$ Since no plateau was evident at the lower concentration range, it is possible that the linear dynamic range observed in Figure 4.2 does not fully cover the binding range. In addition, the inconsistencies in characterization methods for aptamer binding have previously been highlighted, indicating that conventional affinity assays may produce conflicting results, requiring multiple characterization strategies. ${ }^{223}$ 

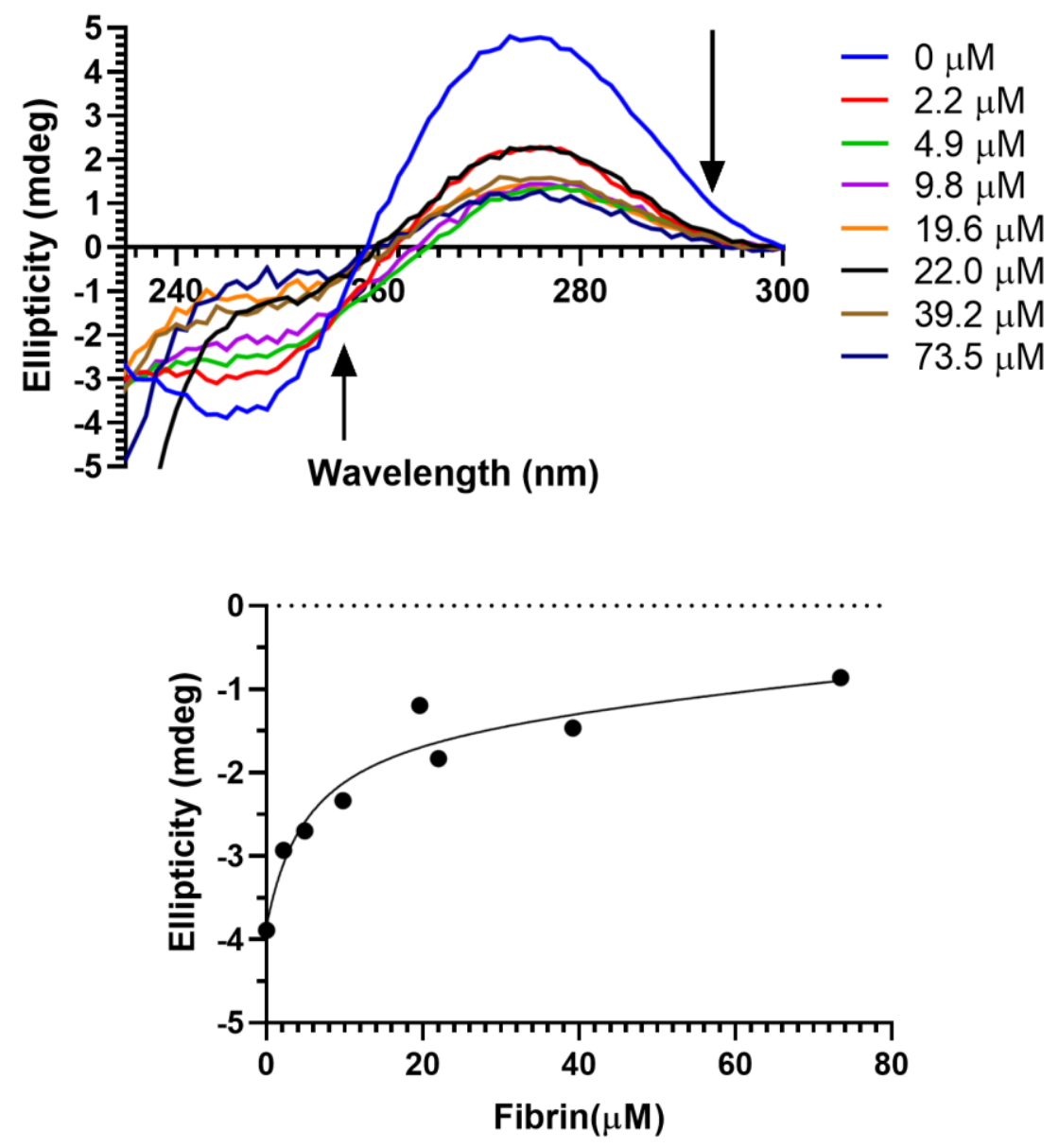

Figure 4.2: CD spectrum of FA in the presence of increasing concentration of fibrin protein, illustrating the change in ellipticity at $245 \mathrm{~nm}$ and $275 \mathrm{~nm}$, associated with aptamer-target binding events.

\subsubsection{Isothermal titration calorimetry}

Isothermal titration calorimetry (ITC) was used to determine the thermodynamic parameters of the binding of iodo-FA to fibrin, which were compared to those of unmodified FA binding to fibrin. This was used to ensure that incorporation of iodine did not disrupt target binding. ITC has been identified as a sensitive technique for evaluation of aptamer-protein interactions through monitoring either the release or the absorbance of heat which occurs upon binding $(\Delta \mathrm{H})$. This is measured through gradual titration of the protein target into the ligand solution. ${ }^{224}$ Binding of both unmodified FA and Iodo-FA was 
monitored over the range of increasing concentrations of fibrin protein. The peaks for enthalpy change, representing the energy changes detected within the system and, therefore, aptamer binding events, were subsequently integrated, constructing a titration curve. In the calculations of $\mathrm{K}_{\mathrm{d}}$, both the concentrations of the ligand and the macromolecule were accounted for. $\mathrm{K}_{d}$ was obtained through the analysis of the titration curve by BindWorks software. The apparent $K_{d}$, based on the concentration of the fibrinogen monomers present within the fibrin network, for unmodified FA was found to be $9.63 \mu \mathrm{M}$ (Figure S4.4). The same analysis was performed for iodo-FA and its apparent $\mathrm{K}_{\mathrm{d}}$ was found to be $9.75 \mu \mathrm{M}$ (Figure S4.5). Since both aptamers were assessed under the same conditions, it is reasonable to deduce that the presence of numerous iodine modifications in iodo-FA does not disrupt the binding. In fact, the binding of both aptamers is extremely similar. As above, this is a considerable loss in binding affinity. Though an apparent plateau was reached at the lower concentration range for both aptamers that were tested, it does not consist of multiple points making up a linear trend. It is conceivable that a more pronounced plateau exists at lower concentrations. In addition, since fibrin was not the target of selection, some loss in binding affinity would be expected. Finally, the polymeric form of the fibrin target may affect the efficacy of the conventional affinity binding assays, which have been identified to have some inconsistencies as it pertains to aptamer-protein binding validation. ${ }^{223}$

\subsubsection{Assessment of CT contrast enhancement capabilities}

\subsubsection{Iodo-FA phantom CT}

The ability of iodo-FA to produce positive enhancement was assessed at $100 \mathrm{kVp}$, with tube current of $150 \mathrm{~mA} \cdot{ }^{225}$ Standard CT scanners are calibrated to a normal HU range 
between -1000 and 1000, with $-1000 \mathrm{HU}$ attributed to air, $0 \mathrm{HU}$ to water, and $1000 \mathrm{HU}$ to bone. ${ }^{143,144}$ A range of concentrations of iodo-FA, with concentrations of $0.1 \mathrm{mM}, 0.25$ $\mathrm{mM}, 0.5 \mathrm{mM}, 0.75 \mathrm{mM}$, and $1 \mathrm{mM}$, was scanned, assessing mean HU for each. Observed and measured contrast was compared to iopamidol, which is a clinical agent that was prepared with the same concentration range. It is immediately apparent that, within the same concentration range, iodo-FA is able to produce visualizable contrast, while iopamidol is not well observed (Figure 4.3A). Iodo-FA demonstrated a concentrationdependent increase in HU, the slope of which was nearly 4-times higher than that of iopamidol (Figure 4.3B). Specifically, the linear slope of iodo-FA was found to be 40.5 $\mathrm{HU} \mathrm{mM}^{-1}$, while iopamidol was $11.3 \mathrm{HU} \mathrm{mM}^{-1}$. This confirmed that iodo-FA could be used as a CT contrast agent and is able to produce more dramatic enhancement when compared to its clinically-available counterparts. ${ }^{226}$ 
A

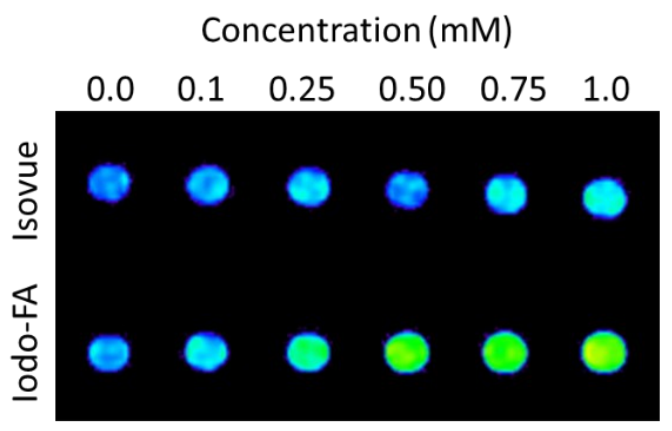

$\mathbf{B}$

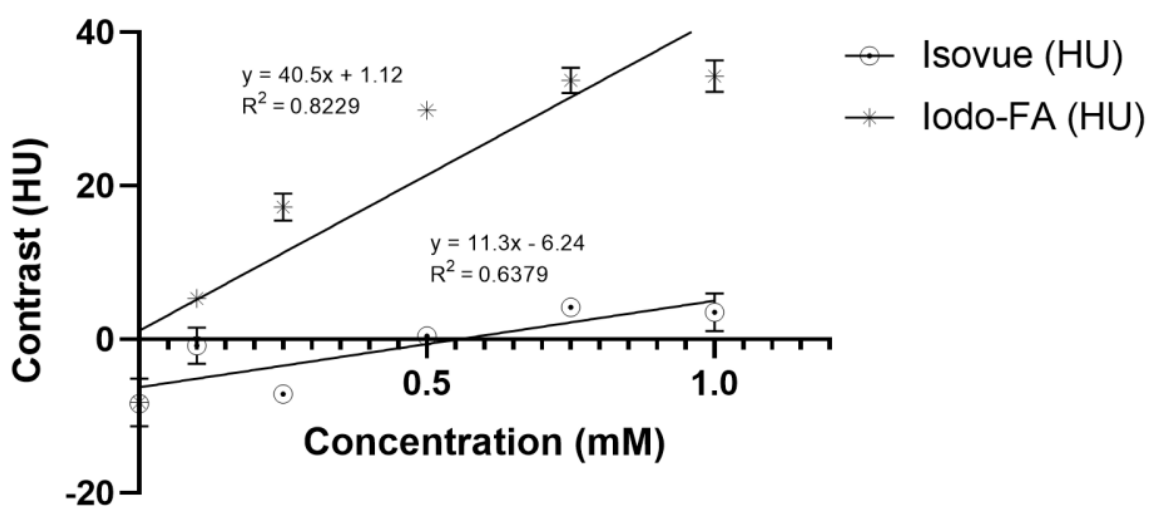

Figure 4.3: (A) CT contrast phantom images of Isovue (iopamidol) and iodo-FA in the same concentration range at $100 \mathrm{kVp}$; (B) Plot of concentration-dependent contrast enhancement (HU), measured with software provided with the CT scanner, for Isovue (iopamidol) and iodo-FA.

\subsubsection{FA-AuNPs phantom CT}

The same assessment was performed with the AuNP-based formulation to ensure that contrast would still be observed at micromolar concentrations. Again, a range of concentrations of FA-AuNPs, with concentrations of $0.1 \mu \mathrm{M}, 0.25 \mu \mathrm{M}, 0.5 \mu \mathrm{M}, 0.75 \mu \mathrm{M}$, and $1 \mu \mathrm{M}$, was scanned $100 \mathrm{kVp}$, with tube current of $150 \mathrm{~mA} .^{225}$ Although the concentration of FA-AuNPs was 1000-times lower, FA-AuNPs were able to produce contrast that was readily observed and considerably higher than the contrast associated with iopamidol (Figure 4.4A). The increase in contrast was directly proportionate to the increase in concentration and the linear slope was calculated to be $563.6 \mathrm{HU}_{\mu} \mathrm{M}^{-1}$ (Figure 4.4B). 
A

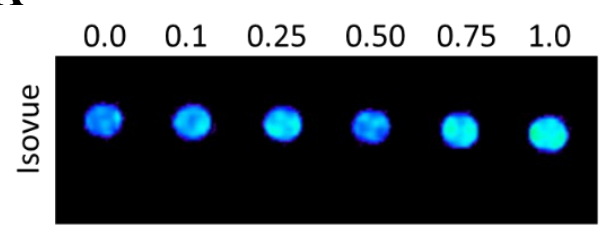

Concentration $(\mu \mathrm{M})$

$\begin{array}{llllll}0.0 & 0.1 & 0.25 & 0.50 & 0.75 & 1.0\end{array}$

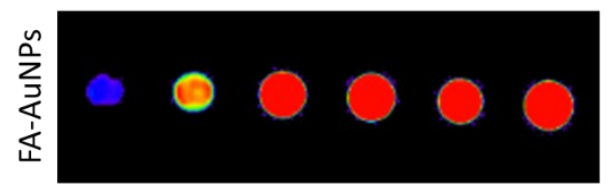

B

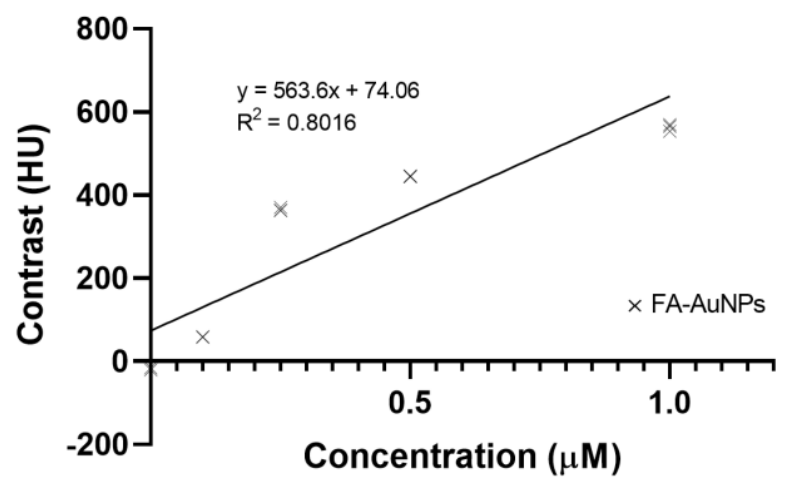

Figure 4.4: (A) CT contrast phantom images of Isovue (iopamidol) and FA-AuNPs, both with increasing concentrations, at $100 \mathrm{kVp}$; (B) Plot of concentration-dependent contrast enhancement (HU), measured with software provided with the CT scanner, for FA-AuNPs.

Overall, both of the formulations developed for molecular targeting of blood clots demonstrated their utility as CT contrast agents. Importantly, more pronounced contrast enhancement was observed with both Iodo-FA and FA-AuNPs. When considering the iodinated aptamer, this was expected as the iodine content of iodo-FA is higher than that of iopamidol, when prepared to the same concentration. In terms of FA-AuNP, the dramatic contrast enhancement at much lower concentration is also reasonable. AuNPs owe their contrasting abilities to the high density of the gold, as well as the overall higher X-ray absorption coefficient. Comparatively, the coefficient of gold is 5.16, while iodine is 1.94 at $100 \mathrm{kVp} .{ }^{75}$ Not accounting for the targeting capabilities, both of these formulations would allow for use of lower concentrations in order to produce observable contrast. This would, in turn, lower the associated toxicity of the currently utilized iodinated contrast media. ${ }^{58}$ In addition, for patients that experience moderate-to-severe allergic reactions to 
iodinated small molecules, clot imaging can be supplemented with AuNP-based contrast agents. ${ }^{27}$

\subsubsection{In vitro assessment with blood clot targets}

\subsubsection{Iodo-FA fluoroscopy (swine blood)}

In vitro fluoroscopy studies were conducted using clotted whole blood from swine ${ }^{152}$ in order to confirm that a swine model can be used for subsequent in vivo experiments and that iodo-FA continues to display good binding affinity in the presence of a complex matrix. Clotted blood was scanned before and after incubation with iodo-FA. Independently, iodo-FA produced visibly dark contrast under standard fluoroscopy settings, while the clot was not visible (Figure 4.5A). When comparing them, the contrast associated with both the clot and the background, while indistinguishable from one another, was brighter than that of iodo-FA (Figure 4.5A). In order for visualizable contrast to develop upon treatment of the clot with iodo-FA, an overnight incubation was required (Figure 4.5A). This is likely associated with the composition of the clot and can be related back to the protocol used in its preparation. That is, when the fibrin network is formed in the presence of red blood cells, the resulting clot is highly viscous. ${ }^{30}$ It is reasonable to infer that this would slow down the rate at which iodo-FA is able to interact with the structural elements of fibrinogen within the clot. Nonetheless, contrast accumulation associated with target-binding of FA to fibrin is evident as the clot becomes visible after an 18-hour incubation.

Visually observed trends were confirmed by measuring the extent of contrast, based on the average pixel intensities (API), using ImageJ software (Figure 4.5B). Three different comparisons were made, using One-way ANOVA test: iodo-FA and blood clot prior to 
incubation $(\mathrm{F}(2,12)=18.98, \mathrm{p}<0.001$ at $\alpha=0.05)$, iodo-FA and clot treated with iodo-FA $(\mathrm{F}(2,12)=114.6, \mathrm{p}<0.0001$ at $\alpha=0.05)$, and iodo-FA and clot treated iodo-FA with reversed settings $(F(2,12)=70.99, p<0.0001$ at $\alpha=0.05)$. In order to verify homogeneity of contrast distribution throughout the clot and the background, contrast was measured in five different, non-overlapping locations. Results suggested that a statistically significant difference exists between treatments. Post-hoc Tukey HSD test confirmed that there is no statistically significant difference between the blood clot and the background $(p=0.96)$, reaffirming that the density of the clot does not contribute to the contrast enhancement related to treatment with iodo-FA. Both, the contrast of the clot and the background, were significantly different from the contrast of iodo-FA $(\mathrm{p}<0.001)$. When comparing the contrast of iodo-FA to the contrast enhancement as a result of the treatment of the clot with iodo-FA, Post-hoc Tukey HSD test revealed that the clot contrast was enhanced to the same level as the contrast achieved by iodo-FA independently, with no significant difference between the two $(\mathrm{p}=0.99)$. Meanwhile, both were significantly different from the contrast observed in the background $(\mathrm{p}<0.0001)$. The same trend was true for the images with reversed settings, with the clots treated with iodo-FA displaying significant enhancement from the background $(\mathrm{p}<0.0001)$. 
$\mathbf{A}$

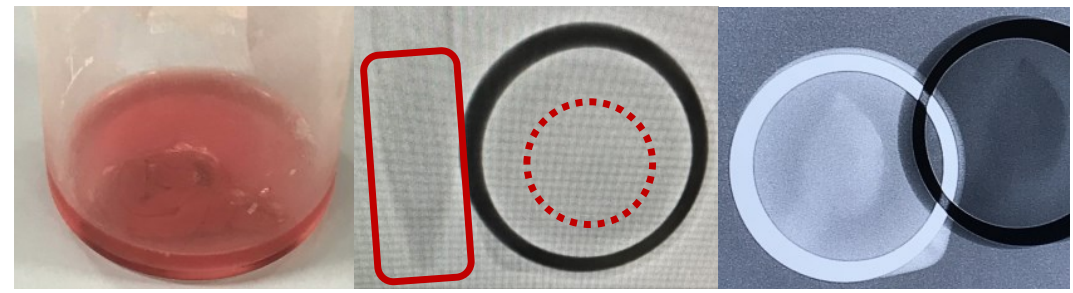

B

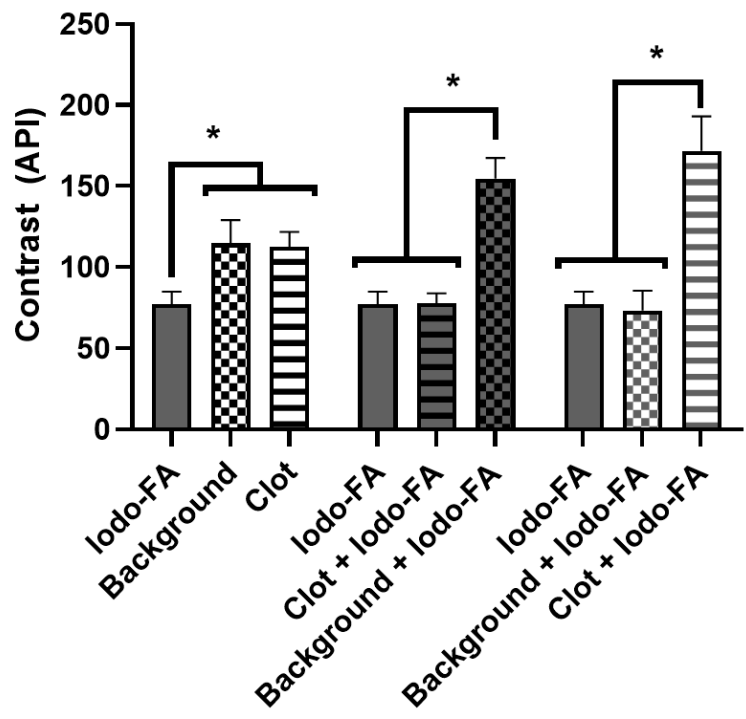

Figure 4.5: (A) Left - clotted pig blood prior to treatment with iodo-FA, middle - in vitro fluoroscopy images of iodo-FA and clotted blood, with iodo-FA visualizable while the clot is not, right - in vitro fluoroscopy images (positive and negative settings) of the clot after treatment with iodo-FA and incubations overnight; (B) Average contrast enhancement (based on API) associated with iodo-FA, blood clot, and observed after treatment of the clot with iodo-FA, measured with ImageJ software and compared using One-way ANOVA test; Tukey post-hoc analyses revealed a significant difference between treatments $(* \mathbf{p}<0.05)$.

\subsubsection{Iodo-FA computed tomography (human blood)}

Further in vitro assessment was performed using computed tomography, as it allows for dual-energy image acquisition to achieve the most pronounced contrast effect through assessment of energy-dependent changes in attenuation. ${ }^{228}$ This assessment was 
performed using clotted human blood, providing optimal binding conditions for FA, which was originally selected against human fibrinogen. ${ }^{28}$ Clotted blood was incubated with iodoFA for 45-minutes to allow for sufficient aptamer-target interaction to occur, resulting in specific accumulation of the contrast at the location of the clot. Incubations with iodoFB139, iopamidol, and PBS were used as controls to assess the specificity of iodo-FA and the distribution of contrast as it pertains to targeted delivery. In order to account for the presence of fibrinogen within the blood stream $(2-5 \mathrm{mg} / \mathrm{mL})^{229}$, two types of iodo-FA treatments were performed, monitoring contrast enhancement of the blood pool and the PBS pool. Notably, observed contrast of iodo-FA treatments in the blood pool and the PBS pool was different. This is likely due to the difference in density and, therefore, attenuation between blood and water, which is still observable on the scale of the contrast enhancement achieved by the given concentrations of iodo-FA. ${ }^{230}$ Importantly, definitive contrast enhancement is present at the location of the clots in treatments with iodo-FA. The accumulation appears to be blood clot-specific, as both the blood pool and the PBS backgrounds remain darker. No such enhancement is observed within the treatments with iodo-FB139, as the contrast is diluted and distributed throughout the entirety of the sample. This is confirmed through comparisons with iopamidol and PBS, neither of which display observable contrast enhancement. In addition, since the clots are not visible in treatments with PBS, we can deduce that the contrast development post treatment with iodo-FA is associated with the presence of the aptamer-targeted contrast agent (Figure 4.6A).

These trends were confirmed to be statistically significant by comparing the measured contrast $(\mathrm{HU})$ of each treatment using One-way ANOVA test $(\mathrm{F}(9,50)=55.2$, $\mathrm{p}<0.001$ at $\alpha=0.05$ ) (Figure 4.6B). Post-hoc Tukey HSD test revealed that there is a 
significant difference between the contrast of the blood clot and the blood pool $(\mathrm{p}<0.001)$ when the clots were treated with iodo-FA in both, the blood pool and the PBS, scenarios. This indicates that the accumulation of iodo-FA at the location of the blood clot is significant enough to allow for differentiation between the possible binding of iodo-FA to circulating fibrinogen and to fibrin. The blood pool and PBS samples were not compared to one another due to the difference in density of these samples. Importantly, no significance was observed between the clots and the blood pools in treatments with iodoFB139, Isovue, and PBS ( $>0.999, p=0.256, p=0.971$, respectively), further confirming that non-specific contrast is simply diluted within the blood pool. 


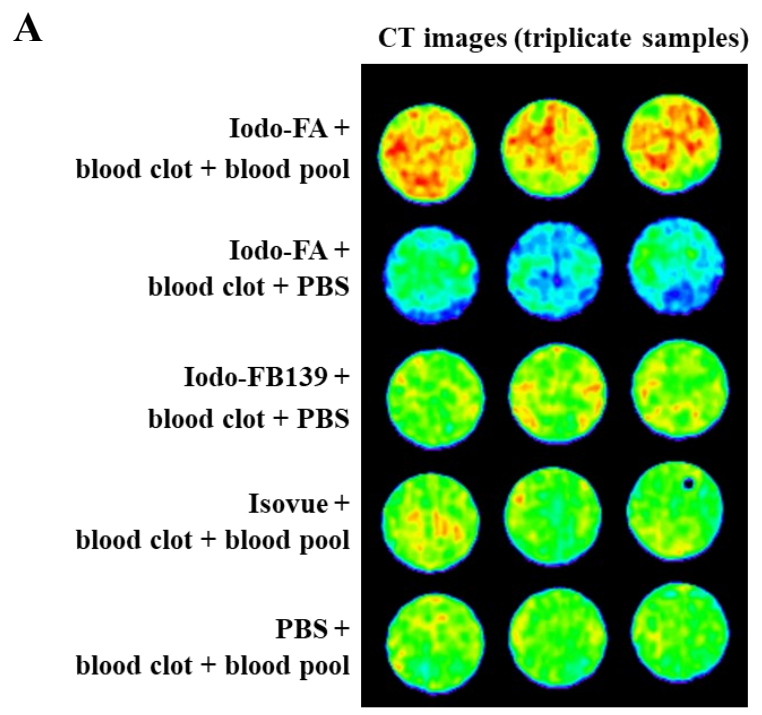

B

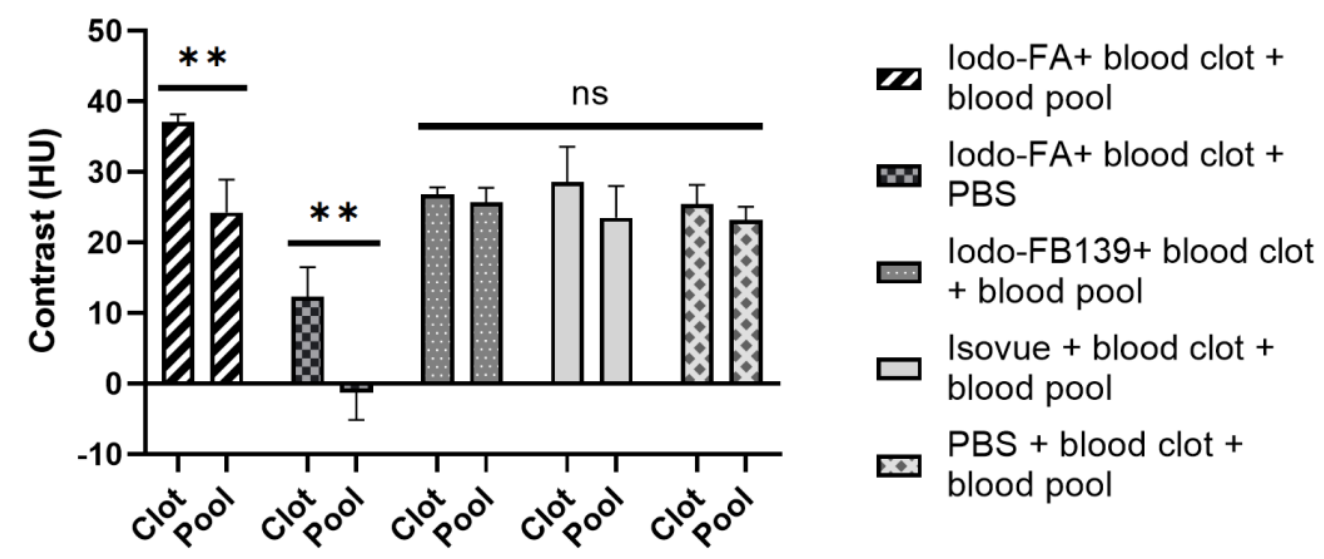

Figure 4.6: (A) In vitro CT scans of blood clots treated with iodo-FA in blood, iodo-FA in PBS, as well as control treatments with iodo-FB139, Isovue (iopamidol), and PBS, all done in triplicate, at $100 \mathrm{kVp}$; (B) Average contrast enhancement (HU) associated with blood clots and the pool of every treatment, measured with CT software provided with the scanner and compared using One-way ANOVA test $(F(9,50)=55.2, p<0.001$ at $\alpha=0.05)$; Tukey post-hoc analyses revealed a significant difference between treatments $(* * \mathbf{p}<0.01)$.

\subsubsection{FA-AuNP computed tomography (human blood)}

The FA-AuNP formulation was also tested in vitro using CT imaging. As above, the incubations were left to equilibrate for 45-minutes with FB139-AuNPs, iopamidol, and 
PBS used as controls. Contrast development in treatments with FA-AuNPs was dramatic in both blood pool and PBS incubations. Based on the contrast capabilities of FA-AuNPs described above (Figure 4.7A), this was expected. It is important to note that, FA-AuNPassociated contrast was observed to this degree due to the presence of high local concentrations of FA-AuNPs at the outer shell of the clots. Interactions of FA with the available fibrinogen structures allowed for this specific accumulation of FA-AuNP to occur. On the other hand, when treated with FB139-AuNP, no such contrast development is observed. Considering the final volume of the sample, FB139-AuNPs are effectively diluted to concentrations that do not produce observable contrast enhancement. Indeed, the levels of attenuation as the result of incubations with iopamidol and PBS were observed to be the same as the control FB139-AuNP treatment (Figure 4.7A).

These results were statistically confirmed, using the average measured contrast (HU) of each treatment, with One-way ANOVA test $(\mathrm{F}(9,50)=93.7, \mathrm{p}<0.0001$ at $\alpha=0.05)$ (Figure 4.7B). Post-hoc Tukey HSD test was used for multiple comparisons. The contrast of the surface of the clot was confirmed to be significantly different from that of the inner clot $(\mathrm{p}<0.001)$ when clots were treated with iodo-FA in both the blood pool and PBS. No statistically significant contrast enhancement was recorded for clots (outer or inner surface) treated with FB139-AuNPs, iopamidol, or PBS ( $\mathrm{p}>0.999$ for each pair-wise comparison). This further confirms how critical ligand-binding associated local contrast accumulation is to development of significant contrast at the location of the clot. 
A

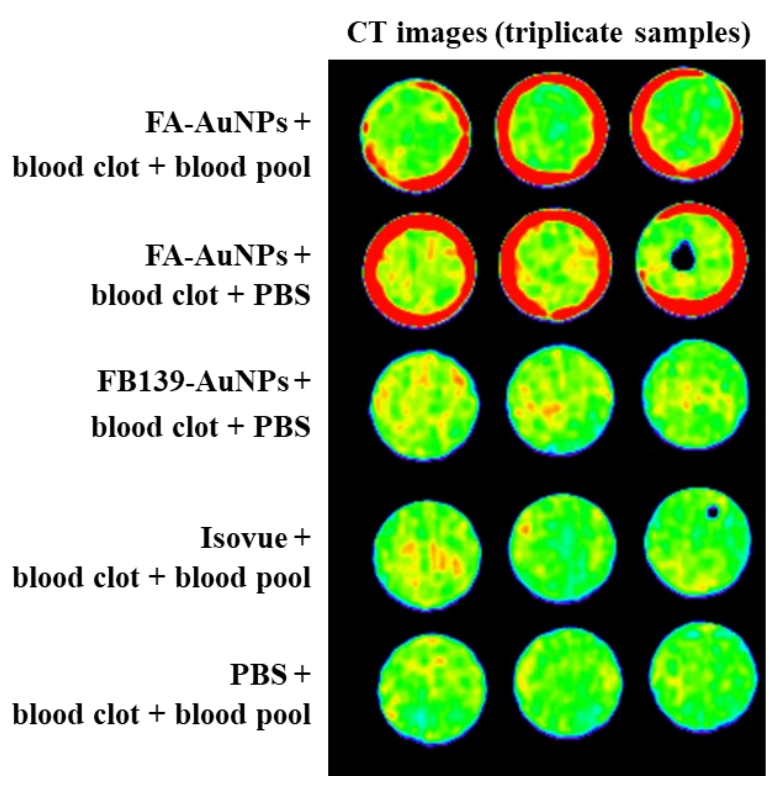

B

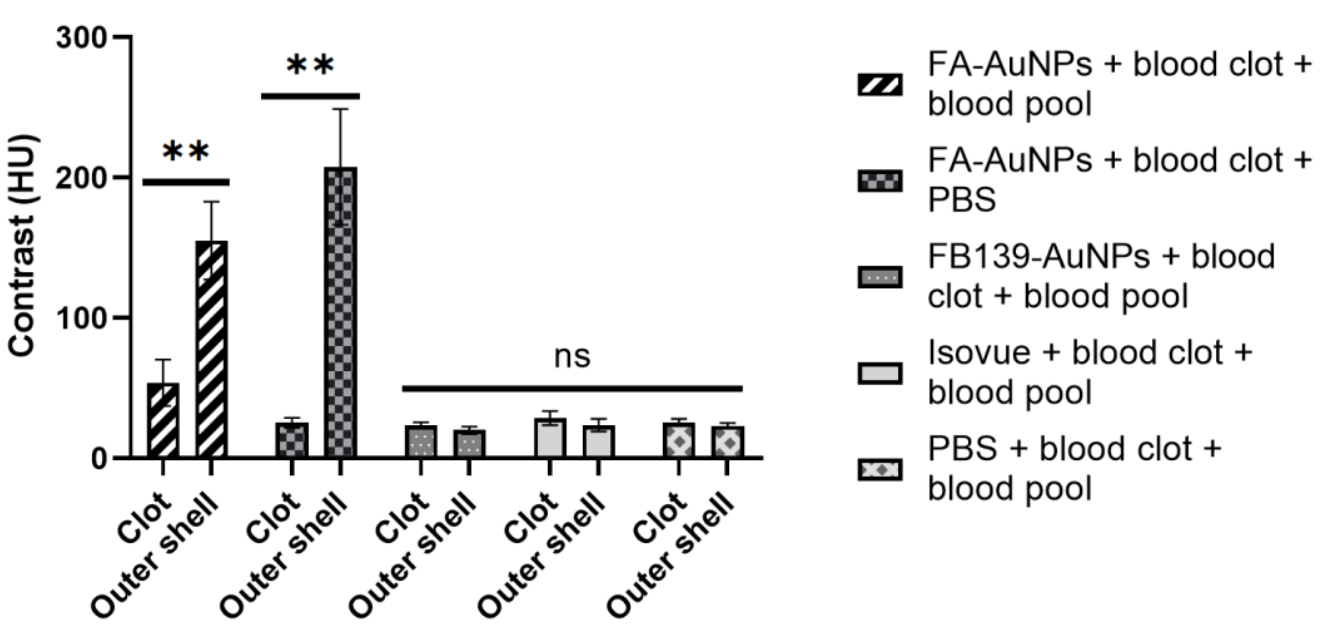

Figure 4.7: (A) In vitro CT scans of blood clots treated with FA-AuNPs in blood, FA-AuNPs in PBS, as well as control treatments with FB139-AuNPs, Isovue (iopamidol), and PBS, all done in triplicate, at $100 \mathrm{kVp}$; (B) Average contrast enhancement (HU) associated with blood clots and the pool of every treatment, measured with CT software provided with the scanner and compared using One-way ANOVA test $(F(9,50)=93.7, p<0.0001$ at $\alpha=0.05)$; Tukey post-hoc analyses revealed a significant difference between treatments $(* * \mathbf{p}<0.01)$. 


\subsubsection{Ex vivo fluoroscopy - iodo-FA (swine model)}

Swine extracranial model was chosen as swine arteries show similarities to human in their vessel size and coagulation parameters. In addition, this model has shown to be useful in translation of methodology into clinical applications. ${ }^{152}$ Right external carotid artery (R ECA) was occluded with a previously prepared clot to mimic a large vascular occlusion. Left carotid artery was used as a control and did not consist of any clots. The occlusion of R ECA was successfully visualized as contrast distribution was no longer observed past the location of the clot (dotted line, Figure 4.8). In the control tissue, left external, internal, and common carotid artery (L ECA, L ICA, L CCA, respectively) were all clearly visible, due to the presence of dark contrast which highlighted the characteristic branching of ECA. ${ }^{152,231,232}$

Again, it is important to consider the characteristics of the clots being used in these experiments. All clots were made using whole blood with regular circulating concentrations of fibrinogen $(2-5 \mathrm{mg} / \mathrm{mL})$, which formed red blood cell (RBC)-rich blood clots. $^{229}$ These clots are not always representative of those implicated in vascular diseases described above, as fibrinogen, an acute phase protein, is recruited to the location of thrombosis, and its concentration is reflective of fibrin network density. ${ }^{26,31,229}$ Most importantly, when considering RBC content of blood clots, it is crucial to account for the direct and complex effect that RBCs exert on the structure and stability of the clot itself. It has been shown that RBC-rich clots decrease fibrin network permeability. ${ }^{229}$ Our previous studies have demonstrated that, in order for visualizable contrast development to occur, the FA conjugates rely on their interaction with the interior components of blood clots. ${ }^{215}$ 

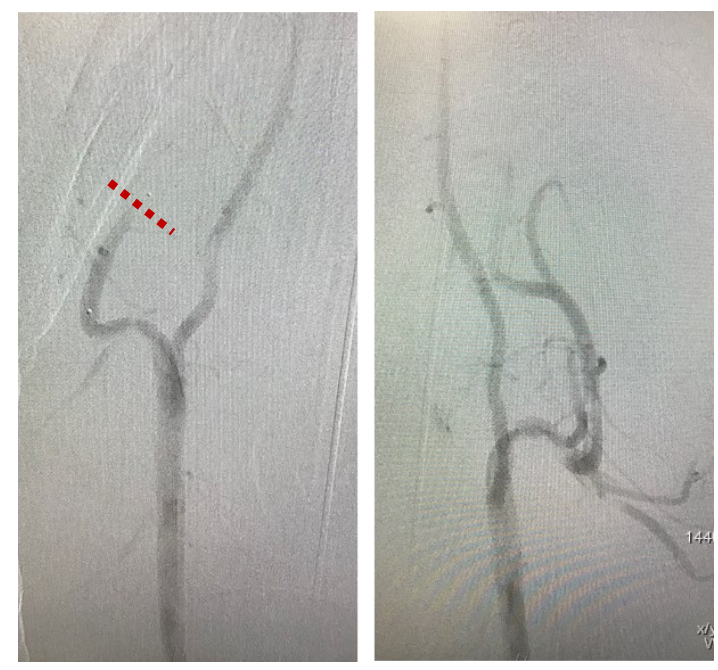

Figure 4.8: In vivo fluoroscopy scan of external carotid arteries with (left) and without (right) a large occlusion disrupting blood and contrast flow. Dashed line indicates the presence of a full occlusion in R ECA.

Following in vivo visualization, $\mathrm{R} \mathrm{CA}$ and $\mathrm{L} \mathrm{CA}$ were harvested during swine necropsy. There was a clearly visible distal dilation in the R ECA, which corresponds with the location of the initial occlusion. No dilations were observed within L CCA control tissue. Since both tissues were dissected immediately following fluoroscopy scans, it was reasonable to expect that some contrast was still remaining within the vessels, based on pharmacokinetics of the predominantly unmodified iodo-FA. ${ }^{233,234}$ That is, iodo-FA was not modified to possess characteristics that would permit its prolonged circulation in the blood stream. ${ }^{235}$ The tissue was fixed overnight in formaldehyde, in order to allow for iodoFA to interact with the clot target. Isolated tissue was subsequently scanned using standard fluoroscopy protocols (Figure 4.9A). In these scans, the colour of the image was inversed to assist the visualization of how iodo-FA works as a brightening contrast. Due to the fixation process, both the control and the experimental tissues hardened, which is necessary for tissue preservation (Figure 4.9B - top). ${ }^{218}$ This hardening was reflected by higher X- 
ray attenuation during the fluoroscopy scan. Both the control and the treated tissues were visible throughout. Despite the overall higher contrast, the dilation bearing the clot was clearly highlighted (Figure 4.9B - bottom). With iodo-FA still present within the blood vessel upon excision, the overnight incubation would allow for the aptamer to slowly diffuse through the vessel and interact with the fibrin target, accumulating to higher local concentrations, making the clot visible.

To confirm this trend, the contrast of the tissues and the clot was measured based on API, using ImageJ, and compared using One-way ANOVA test $(\mathrm{F}(2,12)=43.45$, $\mathrm{p}<0.0001$ at $\alpha=0.01$ ), revealing a statistical difference between treatments (Figure 4.9C). As above, in order to verify homogeneity of contrast distribution throughout the clot and the rest of the tissue, contrast was measured in five different, non-overlapping locations. Multiple comparisons were done using Post-hoc Tukey HSD test in order to confirm whether the contrast developed at the location of the clot was significantly different from the background tissue. Indeed, while the attenuations of the L CCA and the R CCA were not different from one another $(p=0.032)$, both were different when compared to the dilation of the R ECA at the location of the clot $(p<0.0001)$. This reaffirms that, given a sufficient amount of time, iodo-FA is able to produce localized contrast enhancement through ligand-directed binding. 
$\mathbf{A}$
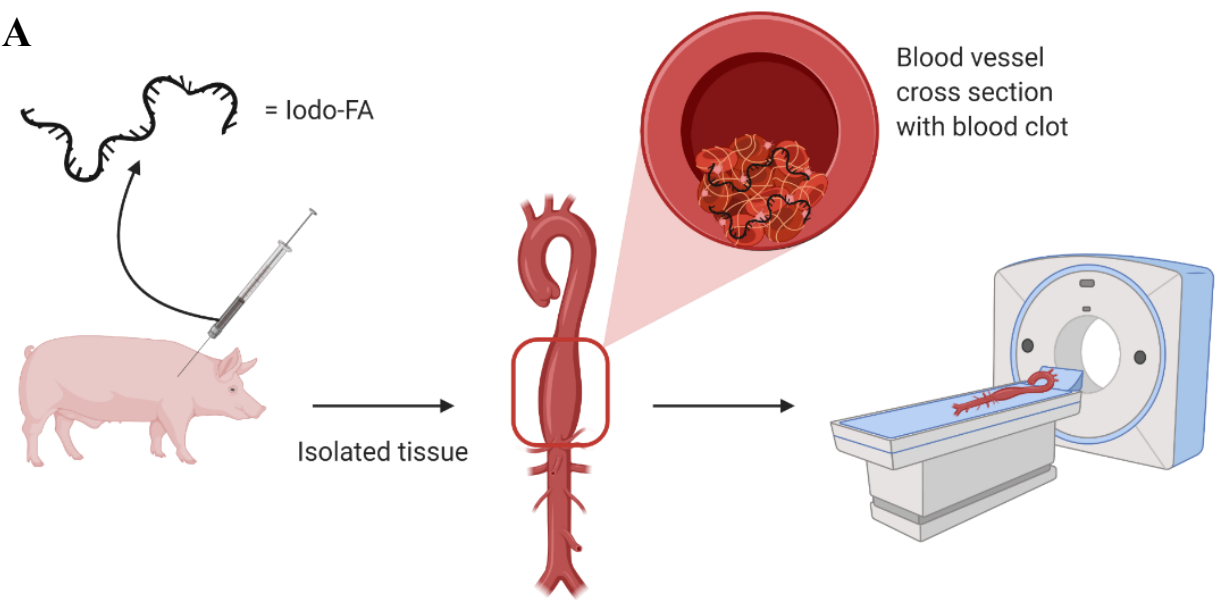

B
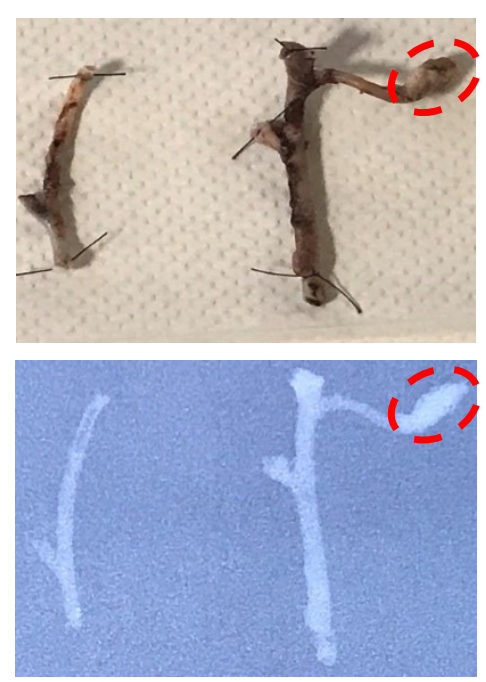

C

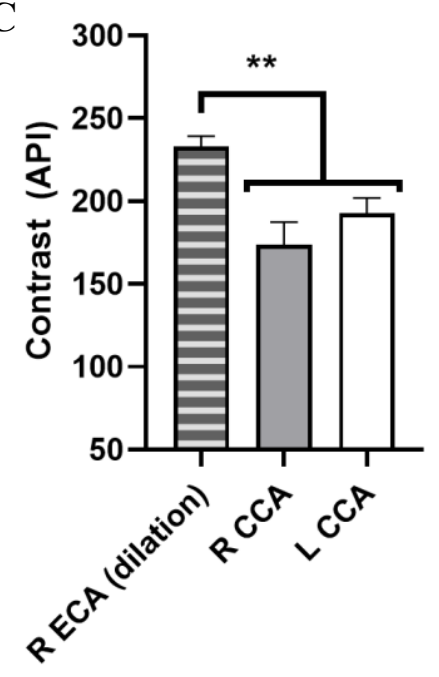

Figure 4.9: (A) Schematic representation of the workflow in preparation for ex vivo fluoroscopy assessment, made in BioRender; (B) Top - harvested external carotid arteries without (left) and with (right) a large occlusion, highlighted in red, bottom - fluoroscopy scan of external carotid arteries without (left) and with (right) a large occlusion, visualized with hyperintense contrast and highlighted in red; (C) Average contrast enhancement (API) measured with ImageJ and compared using One-way ANOVA test $(F(2,12)=43.45, p<0.0001$ at $\alpha=0.01)$. Tukey post-hoc analyses revealed a significant difference between treatments $(* * p<0.01)$. 
A considerable amount of research has gone into improving clinically available contrast media in an effort to circumvent the currently understood limitations associated with nonspecific distribution. Several methods have been proposed, including incorporation of targeting agents and using nanoformulation-based alternatives. As such, it has been well established that the presence of a target-binding ligand allows for molecular imaging of various markers of disease states. In this research, we tested an aptamer that was previously selected to bind a monomeric protein that can be classified as an acute phase protein. ${ }^{26,28}$ This streamlined the development of two different fibrinspecific probes for applications in CT imaging. Both, iodine- and gold- based formulations, iodo-FA and FA-AuNP, respectively, were tested for their ability to produce significant contrast enhancement and were compared against the currently utilized CT contrast material, Isovue (iopamidol). A concentration-dependent increase in X-ray attenuation was achieved. Specifically, iodo-FA was able to produce measurable contrast which was 4times higher than Isovue within the same concentration range. FA-AuNPs, on the other hand, achieved contrast enhancement that was dramatically higher at micromolar concentrations. This indicates that AuNP-based contrast can be much more successful than iodinated contrast.

In testing the ability of FA to target fibrin within both iodo-FA and FA-AuNP formulations, we were able to demonstrate that ligand binding is crucial in the development of clinically observable and significant contrast. Without target-specific aptamer-guided localization, administered contrast was diluted due to non-specific distribution throughout the sample much like the clinical agent. This was true for both iodine- and gold-based formulations. Specifically, in vitro testing allowed for visualization of contrast 
development in the absence of blood flow, which eliminated the need to account for the pharmacokinetics of the predominantly unmodified iodo-FA. ${ }^{233,234}$ Both iodo-FA and FAAuNP successfully targeted blood clots, which resulted in high local contrast concentrations that were quantified. Ex vivo testing confirmed that clots can be delineated through fluoroscopy imaging with the help of iodo-FA. One of the limitations of these formulations is that FA is able to target both, blood fibrinogen and fibrin present within blood clots. As such, in in vitro imaging with FA-AuNPs, it was difficult to differentiate between the blood pool and blood clots, which prevented this formulation from moving into a swine model at this time. Future development of a fibrin-specific aptamer would be recommended to further increase the ability of these formulations to fully define blood clot boundaries through targeting of fibrin meshwork. 


\subsection{Supporting information}

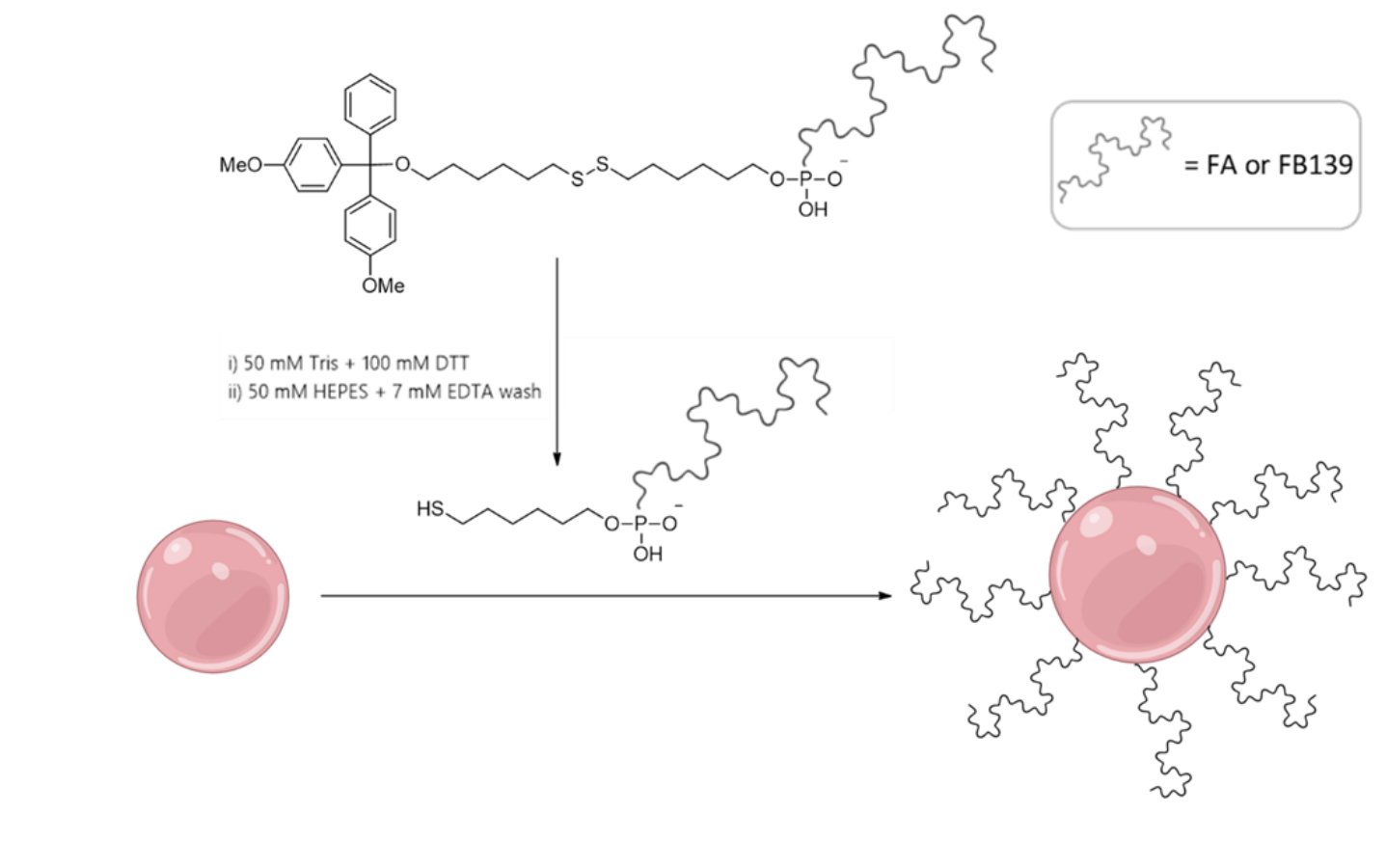

Scheme S4.1: Reaction scheme outlining the cleavage of the thiol-modifier C6 S-S and subsequent conjugation to the surface of AuNPs to produce FA-AuNPs.

\subsubsection{Additional results}

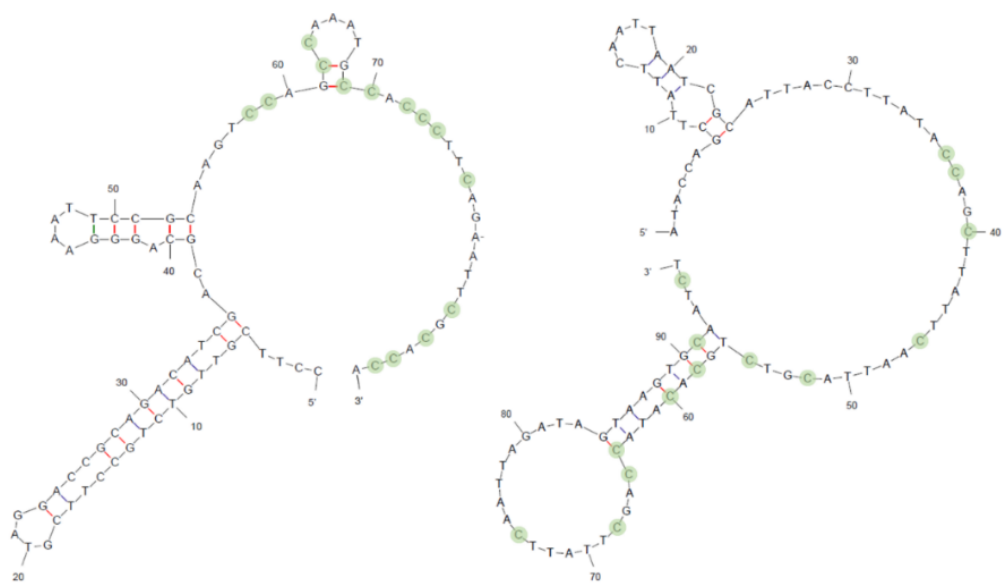

Figure S4.1: Predicted secondary structure of FA (left) with $\Delta G=-9.15 \mathrm{kcal} / \mathrm{mol}$ at $37^{\circ} \mathrm{C}$ and $\mathrm{FB} 139$ aptamer (right) with $\Delta \mathrm{G}=-4.17 \mathrm{kcal} / \mathrm{mol}$ at $37^{\circ} \mathrm{C} ; 5$-iodocystine bases are highlighted in green. Both sequences were folded by Mfold. ${ }^{203}$ 


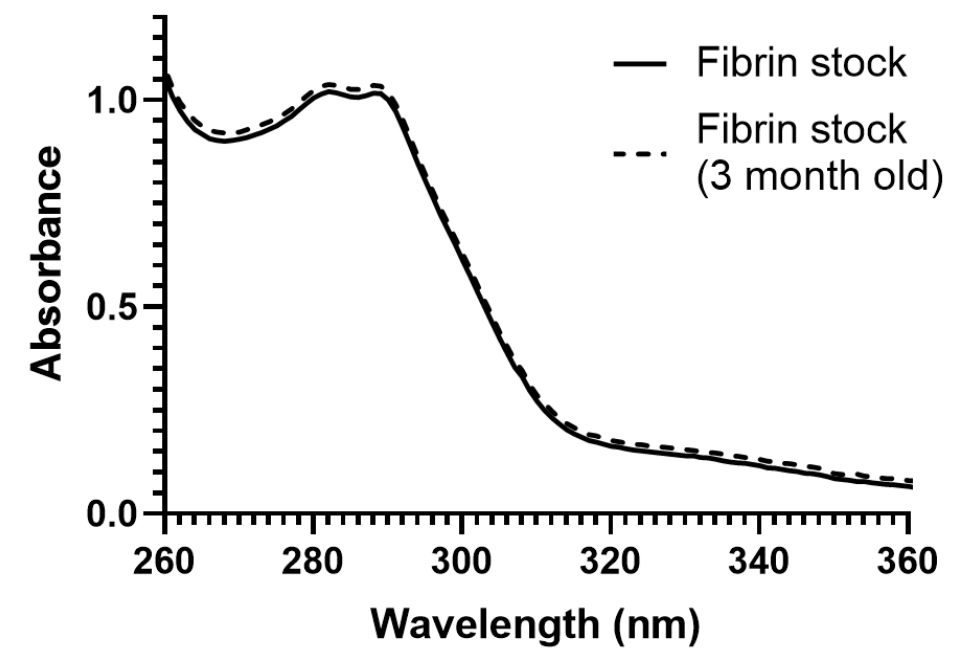

Figure S4.2: UV-Vis spectrum of lyophilized fibrin powder solubilized in $1 \mathrm{M} \mathrm{NaOH}$, assessing stability in solution form after 3 months of storage. The peak at $280 \mathrm{~nm}$ was monitored, corresponding to the absorbance of fibrinogen constituents. 
Table S4.1: One-side total saturation fit results in calculating apparent dissociation constant for FA binding fibrin

\begin{tabular}{c|c}
\multicolumn{2}{c}{ One site -- Total } \\
\hline \multicolumn{2}{c}{ Best-fit values } \\
\hline$B_{\max }$ & 2.525 \\
$K_{d}$ & 5.268 \\
$N S$ & 0.008136 \\
Background & -3.851
\end{tabular}

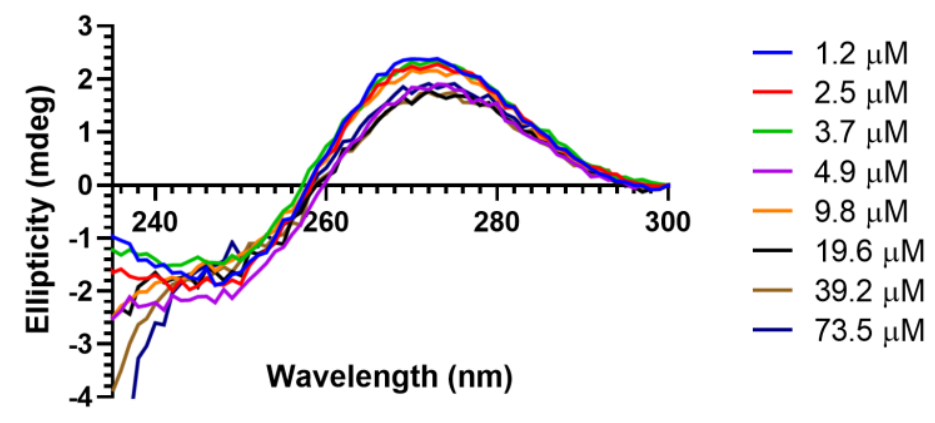

Figure S4.3: CD spectrum of FB139 in the presence of increasing concentration of fibrin protein.
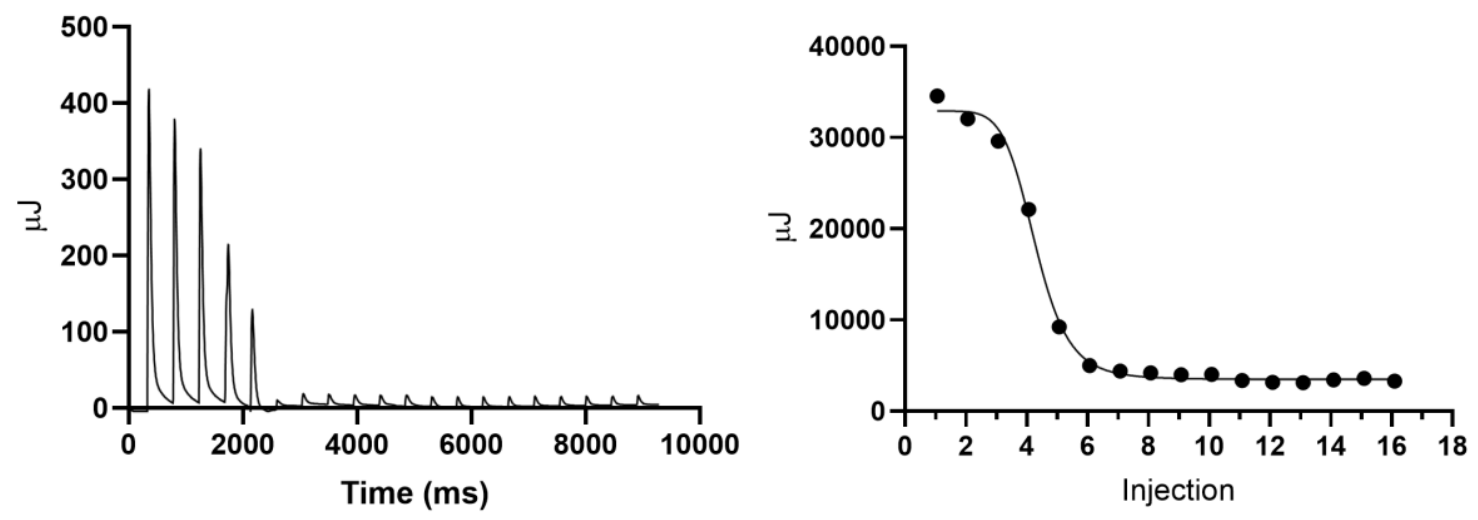

Figure S4.4: ITC thermogram for FA: (Left) heat changes measured during each injection of chemically titrated fibrin target; (Right) binding isotherm of unmodified FA with fibrin target used for calculation of apparent $K_{d}$ in BindWorks. 

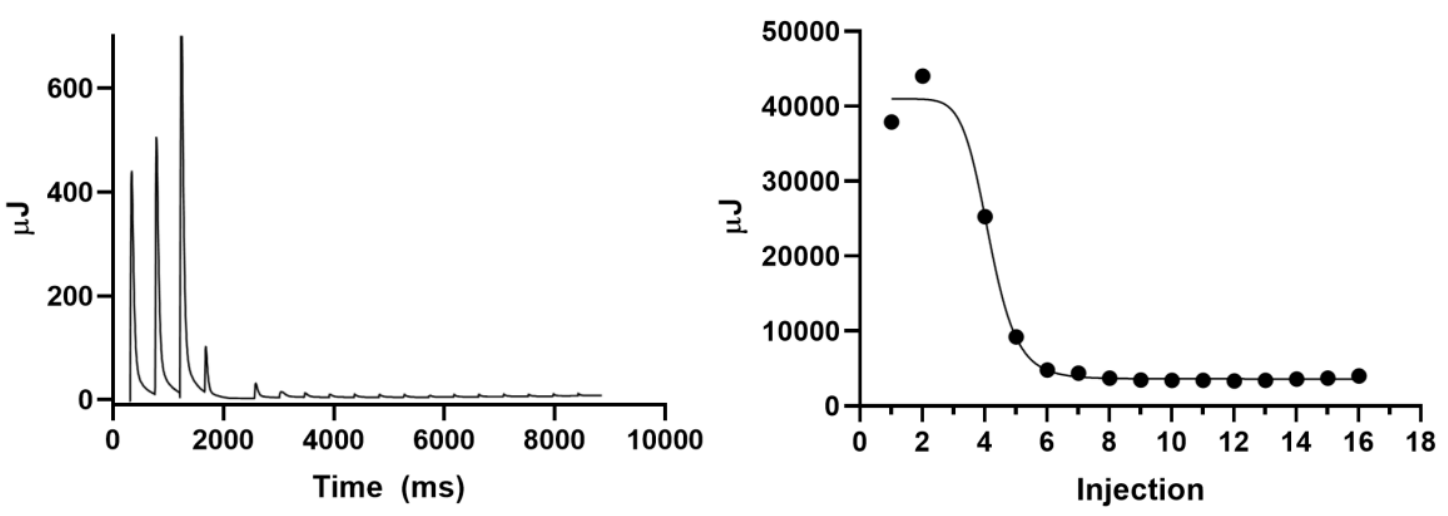

Figure S4.5: ITC thermogram for iodo-FA: (Left) heat changes measured during each injection of chemically titrated fibrin target; (Right) binding isotherm of Iodo-FA with fibrin target used for calculation of apparent $K_{d}$ in BindWorks (note that $1^{\text {st }}$ injection was excluded from analysis) 


\section{Chapter 5: Aptamer functionalized gold-coated iron-oxide nanoparticles as dual imaging agents in MRI and CT}




\subsection{Statement of contribution}

Core-shell nanoparticles were synthesized by A Koudrina, with the help of C Chartrand. Human blood samples were prepared by A Koudrina. MC DeRosa assisted with experimental design. TEM imaging was performed by JJ Wang. All MRI imaging was done by CO Cron, while all CT imaging was done by J O’Brien at the Ottawa Hospital. EC Tsai is the clinical collaborator on this project.

\subsection{Resulting publications}

This chapter is based on an article entitled "Aptamer functionalized gold-coated iron-oxide nanoparticles as dual imaging agents in MRI and CT" submitted for preparation in ACS Nanoscale Advances (Koudrina A, Chartrand C, Cron GO, O’Brien J, Tsai EC, DeRosa MC. RCS Nanoscale Advances. Manuscript ID: NA-COM-09-2020-000781) 


\subsection{Introduction}

The hemostatic system regulates responses of the biological system to injury in a variety of acute events. Coagulation, otherwise known as secondary hemostasis, is necessary in many scenarios, including but not limited to minor injury, trauma or surgery. However, the hemostatic system is delicately balanced, and malfunctions may lead to formation of vascular occlusions which are highly undesirable. Simply said, the formation of blood clots, or thrombi, can be broken down into two categories, comprising of "good clots" and "bad clots". $31,34,236$ This can be directly correlated to acute vascular events, such as strokes. In case of a hemorrhagic stroke, "good clots" act as a seal at the location of the vessel injury. On the other hand, distal or local formation of a "bad clot" can result in an ischemic stroke. ${ }^{153}$ Fibrin, as the main protein constituent of thrombi, is formed during secondary hemostasis and can, therefore, be used as a pathologic biomarker of thrombosis. ${ }^{139}$ The ability to conduct whole body clot assessment has been identified as a solution for streamlined definitive diagnosis ${ }^{48}$ A single contrast material that is applicable towards different imaging modalities would allow for such assessment, while targeting of the said material would allow for molecular imaging of specific components of thrombi. ${ }^{31,48,165}$ Among others, magnetic resonance imaging (MRI) and computed tomography (CT) are the imaging modalities that can be used to diagnose vascular conditions described here. ${ }^{154}$

MRI is governed by the same principles as nuclear magnetic resonance in that it utilizes the ability of non-zero spin nuclei to create a local magnetic field when placed within a strong external magnetic field. Since, within biological systems, hydrogen protons exist in abundance, they are highly susceptible to these effects when placed in a static 
magnetic field. When protons are excited through the application of a radio frequency pulse, their relaxation back to the original lower state is referred to as relaxivity. Relaxation times of all protons are recorded and reconstructed into three-dimensional images, which essentially map the location of water and fat within the biological system. ${ }^{14,140,155}$ Ultrasmall superparamagnetic nanoparticles (USPIONs) $(\sim 3-50 \mathrm{~nm})$ have shown to be effective as T2 contrast agents used to enhance tissue contrast. Though some FDA approved examples exist, such as ferumoxide for liver imaging and ferumoxsil for gastrointestinal imaging, they are not currently used in clinic. ${ }^{139}$ These materials produce hypointense, or dark, contrast by shortening spin-spin relaxation time of nearby protons. Specifically, iron exhibits a magnetic moment due to the presence of unpaired electrons, and, when narrowing the scope to $\mathrm{Fe}_{3} \mathrm{O}_{4}$, superparamagnetism is the result of two different ions $\left(\mathrm{Fe}^{2+}\right.$ and $\left.\mathrm{Fe}^{3+}\right)$ with unequal opposing moments leaving a spontaneous net magnetic moment. The susceptibility difference between the USPIONs and the surrounding medium results in a localized inhomogeneity that is visualized as contrast. ${ }^{140,237}$ Essentially, the bulk effect on the protons provided by nearby water molecules is used to assess pathology, which means that this is inherently mainly based on nonspecific distribution. ${ }^{139}$ However, one major advantage of USPIONs is that their large surface-to-volume ratio allows for administration of lower concentrations. ${ }^{140,175}$

CT utilizes the ability of materials to block ionizing energy. Cross-sectional images are obtained through a combination of X-ray attenuation measurements taken around a single axis of rotation; these images are further processed to provide the final threedimensional reconstruction. X-ray attenuation varies based on the composition of the material being scanned and is measured on the Hounsfield scale. With everything 
normalized to the attenuation of water, assigning the measurement of $0 \mathrm{HU}$, highly dense calcified materials, like bone, will have attenuation values of $1000 \mathrm{HU}$, while air will measure at -1000 HU. ${ }^{143,144}$ Most commonly used CT contrast agents are iodine-based compounds, which are typically made up of a central 2,4,6-triiodinated benzene ring. ${ }^{146}$ Unfortunately, they exhibit high renal toxicity and low imaging time, which has led to a number of investigations towards alternative contrast materials. ${ }^{238}$ The overarching consideration during the selection of an effective contrast agents is that, when the incident $\mathrm{X}$-ray energy is equal to or greater than the binding energy of the K-shell electron of an atom, an increase in absorption coefficient is observed as contrast. ${ }^{143,144}$ With this in mind, gold nanoparticles have been identified as an excellent alternative to the iodine-based contrast agents, as they experience better X-ray attenuation, thereby providing higher contrast enhancement, and they can be easily modified to achieve molecular targeting. ${ }^{239}$

Core-shell nanoparticles (CSN) are composite materials consisting of a core that is different in composition from the shell. ${ }^{238,240,241}$ This affords CSNs a number of properties that gives them utility in a wide range of disciplines, including bioimaging and biosensor applications, targeted drug and gene delivery. ${ }^{238,241}$ A popular example of CSN used in biomedical imaging is superparamagnetic gold-coated iron-oxide nanoparticles. Several advantages of gold-coated iron-oxide nanoparticles $\left(\mathrm{Fe}_{3} \mathrm{O}_{4}-\mathrm{AuNPs}\right)$ have been identified, including their decreased cytotoxicity, increased biocompatibility, and ability to be surface functionalized. ${ }^{238}$ The inert gold coating is the main contributor to such diverse characteristics, while the iron oxide core possesses superparamagnetic. ${ }^{238,239}$ In fact, functional complementarity of gold and iron oxide allows for these types of nanoparticles to be used in multimodal imaging, including MRI and CT. ${ }^{238}$ Multimodality allows for 
unity of imaging techniques that, when utilized separately, suffer from variable limitations, but when combined, provide congruity based on complementary strengths. MRI offers high spatial resolution, and good soft tissue contrast, while providing both anatomical and functional information, which are all limitations of CT, whereas CT presents highsensitivity, which is a limitation of MRI. ${ }^{239}$ This, in combination with the aforementioned advantages of composite nanomaterials like $\mathrm{Fe}_{3} \mathrm{O}_{4}-\mathrm{AuNPs}$, not only provides an opportunity for lowering the potential toxicity associated with clinically used contrast, but also allows for optimization of distribution and residence time in tissues and, therefore, more precise imaging. ${ }^{57,238}$ In addition, even non-functionalized nanoparticles are able to passively target tumor vasculature to a limited degree, through enhanced permeability and retention (EPR) effect, by preferentially accumulating in tumor vessels. ${ }^{66,67,242}$ To expand their application and achieve highly resolved targeted imaging, $\mathrm{Fe}_{3} \mathrm{O}_{4}$-AuNPs have been modified with targeting elements geared towards specific molecular markers. ${ }^{175,238,239} \mathrm{~A}$ number of targeting ligands exist and have been researched; however, aptamers offer a several advantageous characteristics. ${ }^{210}$

Aptamers are oligonucleotide-based recognition elements that have been reported for a wide range of targeting. They offer a number of advantages over the more established affinity tools such as antibodies, including superior stability, ability to be chemically synthesized and modified, while remaining non-immunogenic and non-toxic. ${ }^{5-7,12,13}$ These attractive properties make aptamers an excellent candidate for use in functionalization of CSN. Such recent example was outlined by Azhdarzadeh et al., where SPIONs were coated with gold and modified with an aptamer, MUC-1, for targeting of aberrant glycosylated form of mucin on tumor cell surface of colon cancer. ${ }^{243}$ The gold coating was used for its 
high photothermal conversion efficiency, as well as for its ability to form gold-sulfur bond utilized to conjugate MUC-1, while the therapeutic effect was monitored with the help of SPIONs. They were able to show that aptamer-functionalized nanoparticles experienced a significantly higher uptake than their counterparts, confirming the utility of aptamers in targeted theranostics. ${ }^{243}$

We hypothesized that, using a fibrinogen aptamer (FA)-functionalized gold-coated iron-oxide nanoparticle-based contrast agent would allow for fibrin targeting. As such, specific accumulation of this material would streamline sensitive assessment of thrombi in T2-weighted MRI or CT, simultaneously lowering required injection concentrations. The feasibility of using molecular imaging of fibrin with targeted CSN was assessed in vitro using T2-weighted MR and CT imaging. FA is an aptamer that was previously selected for human fibrinogen by a different group. The candidate chosen for this work was one that did not possess any modifications while exhibiting good binding affinity towards fibrinogen. ${ }^{28}$ Our group previously demonstrated that FA can be used to bind the polymeric form, fibrin, targeting of which allowed to establish significant contrast enhancement of blood clots. ${ }^{215}$

\subsection{Materials and methods}

\subsubsection{Chemicals and instruments}

Iron (III) acetylacetonate $(\mathrm{Fe}(\mathrm{acac}) 3), 99 \%$, phenyl ether (99\%), oleic acid (99\%), oleylamine (70\%), anhydrous sodium citrate (99\%), 4-dimethyl(amino)pyridine (99\%), hexanes, and absolute ethanol were purchased from SigmaAldrich (Oakville, ON, Canada). 1,2-hexadecanediol was purchased from TCI (Portland, Oregon, USA). Gold (III) acetate $\left(\mathrm{Au}(\mathrm{ac})_{3}\right)$ was purchased from Alpha Aesar (Haverhill, Massachusetts, USA). 300 mesh x 
$83 \mu \mathrm{m}$ pitch copper TEM grids were purchased from Electron Microscopy Sciences (Hatfield, Pennsylvania, USA). All glassware used for the $\mathrm{Fe}_{3} \mathrm{O}_{4}$-AuNP synthesis was washed with aqua regia (3:1 mixture concentrated $\mathrm{HCl} / \mathrm{HNO}_{3}$ ) and rinsed thoroughly with deionized water. The glassware was then rinsed with acetone before being dried in an overnight or until used for synthesis. Unless otherwise stated, all synthetic procedures were performed using standard Schlenk techniques under an argon (5.0, 99.999 \%) atmosphere.

All DNA was synthesized using Bioautomation MerMade 6 oligonucleotide synthesizer (Plano, TX, USA), using standard phosphoramidite chemistry. Phosphoramidites, synthesis reagents, and thiol C6 S-S modifier were all purchased from Glen Research (Sterling, VA, USA). Ultra-High Purity 5.0 argon was purchased from Praxair Canada (Mississauga, ON, Canada). Standard support columns and acetonitrile were purchased from BioAutomation (Plano, TX, USA). All DNA was purified using Glen-Pak cartridges purchased from Glen Research (Sterling, VA, USA) using the appropriate protocol for the modifier used during the synthesis. All purified DNA was sent to Novatia, LLC (Monmouth Jct, NJ, USA) in order to obtain molecular weight confirmation of the products by ESI-MS. De-salting and separation after the cleavage of the disulfide bond was done using Amicon-Ultra $0.5 \mathrm{~mL} 3 \mathrm{kDa}$ cut-off ultra-centrifugal units purchased from Fisher Scientific Canada (Ottawa, ON, Canada).

Blood was obtained from The Ottawa Hospital Research Institute with approval from the Ottawa Health Science Network Research Ethics Board, OHSN-REB (application \# 20150544-01H). All MR Imaging was done using an animal GE/Agilent MR901 Discovery 7T MRI Scanner (The University of Ottawa, Preclinical Imaging Core). All CT 
Imaging was done using General Electric HD 750 Discovery CT Scanner (The University of Ottawa).

\subsubsection{Preparation of iron oxide $\left(\mathrm{Fe}_{3} \mathrm{O}_{4}\right)$ nanoparticles}

$\mathrm{Fe}_{3} \mathrm{O}_{4}$ nanoparticle seeds (as well as the final $\mathrm{Fe}_{3} \mathrm{O}_{4}$-AuNPs) were synthesized using a previously established protocol by Smith et al. ${ }^{241} \mathrm{In}$ brief, $0.71 \mathrm{~g} \mathrm{Fe}(\mathrm{acac})_{3}(2$ mmol) was mixed in $20 \mathrm{~mL}$ of phenyl ether with $2 \mathrm{~mL}$ of oleic acid $(\sim 6 \mathrm{mmol})$ and $2 \mathrm{~mL}$ of oleylamine ( 4 mmol) under argon atmosphere with vigorous stirring. Once dissolved, $2.58 \mathrm{~g}$ of 1,2-hexadecanediol $(10 \mathrm{mmol})$ was added into the solution and a water-cooled condenser was affixed to the flask. The final solution was slowly heated to $210^{\circ} \mathrm{C}$ (without exceeding this temperature) and refluxed for $2 \mathrm{~h}$. After $2 \mathrm{~h}$, a colour change was observed with bright red suspension turning dark brown. The reaction solution was cooled to room temperature under argon overnight and used directly in the subsequent step without separation.

\subsubsection{Direct coating of $\mathrm{Fe}_{3} \mathrm{O}_{4}$ nanoparticles to produce $\mathrm{Fe}_{3} \mathrm{O}_{4}-\mathrm{AuNPs}_{4}$}

$10 \mathrm{~mL}$ of the phenyl ether reaction solution of $\mathrm{Fe}_{3} \mathrm{O}_{4}$ nanoparticles $(\sim 0.33 \mathrm{mmol}$ $\mathrm{Fe}_{3} \mathrm{O}_{4}$ ) were added to $30 \mathrm{~mL}$ of phenyl ether with vigorous stirring. To that, $0.83 \mathrm{~g}$ of $\mathrm{Au}(\mathrm{ac})_{3}(2.2 \mathrm{mmol}), 3.1 \mathrm{~g}$ of 1,2-hexadecanediol $(12 \mathrm{mmol}), 0.5 \mathrm{~mL}$ of oleic acid $(\sim 1.5$ $\mathrm{mmol})$, and $3 \mathrm{~mL}$ of oleylamine $(\sim 6 \mathrm{mmol})$ were added quickly. The mole ratio of the $\mathrm{Au}$ precursor to the iron oxide nanoparticles was approximately 7:1. Under argon atmosphere and with vigorous stirring, the reaction solution was heated slowly to $180-190{ }^{\circ} \mathrm{C}$ (10 ${ }^{\circ} \mathrm{C} / \mathrm{min}$ ) and was maintained at this temperature under reflux for $3 \mathrm{~h}$. The reaction solution was cooled to room temperature under argon overnight. $5 \mathrm{~mL}$ of the cooled solution was combined with $15 \mathrm{~mL}$ of ethanol and agitated gently, resulting in visible aggregation of 
nanoparticles. The aggregates were magnetically separated by placing the flask on top of 6 rare earth magnets for 5-10 mins. The resulting supernatant was decanted as waste and the precipitated nanoparticles were washed three times with $15 \mathrm{~mL}$ of absolute ethanol. The nanoparticles were de-dispersed in $10 \mathrm{~mL}$ of hexanes, containing equal parts oleic acid and oleylamine ( $\sim 75 \mathrm{mM}, 0.25 \mathrm{~mL})$. This procedure was repeated several times in order to obtain the necessary volume of $\mathrm{Fe}_{3} \mathrm{O}_{4}$-AuNPs.

\subsubsection{Sodium citrate ligand exchange and conjugation with aptamer}

The following steps were not performed under argon atmosphere. $5 \mathrm{~mL}$ of previously prepared $\mathrm{Fe}_{3} \mathrm{O}_{4}$-AuNPs in hexanes was precipitated as above, by magnetic separation using $15 \mathrm{~mL}$ of ethanol. The nanoparticles were washed three times with $15 \mathrm{~mL}$ of absolute ethanol and redispersed in $3 \mathrm{~mL}$ of $1 \mathrm{M}$ TMAOH containing $0.2 \mathrm{~g}$ of sodium citrate. The $\mathrm{pH}$ of solution was slowly adjusted to approximately 6.5 with dilute $\mathrm{HCl}$. The solution was sonicated at room temperature for 15 mins. The nanoparticles were subsequently collected using a magnet and redispersed in $5 \mathrm{~mL}$ of Milli-Q deionized water and sonicated for a further 5 mins at room temperature.

Thiol-FA/FB139 (60 nmol) was vortexed in $75 \mu \mathrm{L}$ of $50 \mathrm{mM}$ Tris $(\mathrm{pH} 8.4)$ containing $100 \mathrm{mM}$ dithiothreitol (DTT) at ambient temperature for 30 minutes in order to cleave the 5'-disulfide in preparation for conjugation with $\mathrm{Fe}_{3} \mathrm{O}_{4}$-AuNPs. Cleaved FA/FB139 was washed using BioRad Micro Bio-Spin Chromatography columns, using EDTA buffer (7 mM, pH 7.0) and subsequently de-salted using Amicon Ultra $0.5 \mathrm{~mL}$ Centrifugal Filters with HEPES buffer (50 mM, 7 mM EDTA, pH 7.0). An aliquot of the final solution of $\mathrm{Fe}_{3} \mathrm{O}_{4}$-AuNPs $(100 \mu \mathrm{L})$ was then combined with $100 \mu \mathrm{L}$ of thiolated (cleaved, $100 \mu \mathrm{M}$ ) FA/FB139. This was shaken overnight to allow for surface- 
functionalization. In order to remove any unbound sequences, FA/FB139- $\mathrm{Fe}_{3} \mathrm{O}_{4}-\mathrm{AuNPs}$ were collected on a rare earth magnet, decanting the remaining solution. They were subsequently redispersed in deionized water and sonicated for 5 mins at room temperature.

\subsubsection{Characterization of $\mathrm{Fe}_{3} \mathrm{O}_{4}-\mathrm{AuNPs}$}

UV-Vis absorption characterization of the $\mathrm{Fe}_{3} \mathrm{O}_{4}$-AuNPs was performed using a Cary 300 Bio UV-Visible spectrophotometer (Varian, Santa Clara CA). Peaks at $404 \mathrm{~nm}$ and $530-540 \mathrm{~nm}$ corresponded to the $\mathrm{Fe}_{3} \mathrm{O}_{4}$-core and thin Au-coating, respectively. Concentrations were calculated using molar extinction coefficients of $\mathrm{Fe}_{3} \mathrm{O}_{4}$-AuNPs were also analyzed at every step of the synthesis using Transmission Electron Microscopy taken with a FEI Technai G2 F20 TEM at the Carleton University Nano-imaging Facility, with a field emission source at a voltage of $200 \mathrm{kV}$ using Gatan Microscopy Suite 2V. All images

were taken on dry 300 mesh $\times 83 \mu \mathrm{m}$ pitch carbon coated copper TEM grids at room temperature. Grids were prepared by placing $4 \mu \mathrm{L}$ of $\mathrm{Fe}_{3} \mathrm{O}_{4}$-AuNP (in various solvents) directly on top, followed by minimum $4 \mathrm{~h}$ of drying.

\subsubsection{In vitro T2-weighted MRI (human blood)}

Whole human blood was clotted using a previously established thrombectomy protocol. ${ }^{152}$ In brief, $1 \mathrm{~mL}$ of $\mathrm{CaCl}_{2}$-thrombin solution was prepared using $0.252 \mathrm{M} \mathrm{CaCl}_{2}$ and $100 \mathrm{U}$ of thrombin. This solution was subsequently mixed with $5 \mathrm{~mL}$ of whole blood and left to form a clot at room temperature for 30 minutes. The large clots were sectioned into smaller clots, approximately $1 \mathrm{~cm}$ by $1 \mathrm{~cm}$ in size. Each blood clot was combined with $1 \mathrm{~mL}$ of either heparinized blood or PBS buffer, $\mathrm{pH}$ 7.4. To each sample, $100 \mu \mathrm{L}$ of $60 \mathrm{nM}$ FA- $\mathrm{Fe}_{3} \mathrm{O}_{4}$-AuNPs (or $\mathrm{FB} 139-\mathrm{Fe}_{3} \mathrm{O}_{4}$-AuNPs for control treatments) was injected and left to incubate for 45 mins. T2-weighted scans ( 7 Tesla, echo time $=42 \mathrm{~ms}$, repetition time $\geq 6 \mathrm{~s}$ ) 
were performed over a 20-minute span. Contrast enhancement was analyzed using ImageJ software.

\subsubsection{In vitro CT imaging (human blood)}

Blood clots were produced from human blood using the same protocol as in section 5.4.6. Each blood clot was combined with $1 \mathrm{~mL}$ of either heparinized blood or PBS buffer, $\mathrm{pH} 7.4$, and samples were set up in triplicate. To each sample, $100 \mu \mathrm{L}$ of $40 \mathrm{nM} \mathrm{FA}-\mathrm{Fe}_{3} \mathrm{O}_{4}-$ AuNPs (or FB139- $\mathrm{Fe}_{3} \mathrm{O}_{4}-\mathrm{AuNPs}$ for control treatments) was injected and left to incubate for 45 mins. All CT Imaging was done using dual energy protocols at $80-140 \mathrm{kVp}$.

\subsection{Results and discussion}

\subsubsection{Synthesis of $\mathrm{FA}-\mathrm{Fe}_{3} \mathrm{O}_{4}-\mathrm{AuNPs}$}

Multifunctional nanoparticle-based systems have previously shown utility in cancer diagnosis and treatment, with magnetic nanoparticles $\left(\mathrm{Fe}_{3} \mathrm{O}_{4} \mathrm{NPs}\right)$ at the forefront of research towards utilization in biomedical fields. Most often, magnetic nanoparticles are designed to achieve multifunctionality through their inherent targeting, imaging, and therapeutic capabilities. ${ }^{244}$ A recent review, Zamay and colleagues outlined the advantages of using aptamers as functional coating of magnetic nanoparticles to achieve active

targeting. ${ }^{245}$ A recent example of such formulation was outlined by Zhong et al., using an endoglin-targeting aptamer, mEND, to selectively deliver $\mathrm{Fe}_{3} \mathrm{O}_{4}$ nanoparticles to the surface of neovascular endothelial cells of hepatocellular carcinoma. This probe was confirmed to have excellent targeting and contrasting abilities in vitro and in vivo, while being highly biocompatible..$^{92}$ 
Unfortunately, a number of synthesis-related constraints have been identified. Among other parameters, it is important but challenging to control for the overall size and monodispersity. Magnetic properties and passive accumulation of these nanoparticles are reserved for those that are smaller than $50 \mathrm{~nm}$ in diameter. ${ }^{246,247}$ However, not only are $\mathrm{Fe}_{3} \mathrm{O}_{4}$ NPs relatively unstable, which leads to aggregation, they also elicit toxic effects within biological systems. Several methods of functionalization to increase biocompatibility have been proposed. Nevertheless, this is difficult to implement, as $\mathrm{Fe}_{3} \mathrm{O}_{4}$ NPs are not compatible with many surface chemistry modifications, and furthermore, it is tricky to control the uniformity of the coating. ${ }^{245,248}$ It is well understood that gold-coating provides desirable characteristics to $\mathrm{CSN}$, increasing stability, biocompatibility, and allowing for easy surface functionalization with a targeting ligand. ${ }^{248}$ Using the previously optimized method by Smith et al., $\mathrm{Fe}_{3} \mathrm{O}_{4}$-core was synthesized and coated with a thin layer of Au to allow for FA to be directly incorporated through the S-Au bond. Briefly, $\mathrm{Fe}(\mathrm{acac})_{3}$ was reduced in the presence of capping agents to produce spherical $\mathrm{Fe}_{3} \mathrm{O}_{4}$ seeds (Figure S5.1). Subsequently, $\mathrm{Au}\left(\mathrm{CH}_{3} \mathrm{COO}\right)_{3}$ was reduced and gold was deposited on the surface of $\mathrm{Fe}_{3} \mathrm{O}_{4}$ NP (Figure S5.2). The final CSN were transferred to $\mathrm{diH}_{2} \mathrm{O}$ and re-capped. Disulfide-modified FA was first reacted with dithiothreitol in order to cleave the disulfide bond and liberate a free reactive thiol group. It was then combined with the final $\mathrm{Fe}_{3} \mathrm{O}_{4}$ AuNPs to achieve FA-functionalized CSN (Figure S5.3, Scheme S5.1). ${ }^{241,249}$ Since a number of washes were required at each step of the synthesis and involved collection of the nanoparticles on rare earth magnets, it was confirmed that neither the gold-coating, nor the aptamer-conjugation perturbed the magnetic properties. This is crucial for providing contrast enhancement in T2-weighted MRI (magnetic properties confirmed in Figure 
S5.5). ${ }^{248}$ The structure and morphology of each synthesized component was confirmed by transmission electron microscopy (TEM) (Figure S5.1-S5.3). Synthesized $\mathrm{Fe}_{3} \mathrm{O}_{4}$-AuNPs and $\mathrm{FA}-\mathrm{Fe}_{3} \mathrm{O}_{4}$-AuNPs were quantified by UV-Vis absorption spectroscopy, monitoring for peaks in the 535-550 $\mathrm{nm}$ range, corresponding to the gold coating, and 260, corresponding to DNA (Figure S5.4). ${ }^{250}$ The final concentration was calculated to be $60 \mathrm{nM}$ based on the gold-coating and $95 \mu \mathrm{M}$ based on the aptamer. Though these techniques were useful in this context, future studies utilizing inductively coupled plasma mass spectrometry would be useful in identification of elemental mass size distribution. ${ }^{251}$

\subsubsection{In vitro T2-weighted MRI (human blood)}

The binding of FA to the fibrin polymer was previously confirmed by our own group. Briefly, binding is immediate upon interaction of FA with fibrin, but requires approximately 10 mins to accumulate to significant amounts. This is clinically relevant, on the timescale of a typical MRI scan. In addition, we were able to confirm that the aptamertargeted gadolinium contrast agent was capable of penetrating the fibrous network, producing significant hyperintense contrast throughout the fibrin clot in T2-weighted MRI. ${ }^{215}$ This formulation was designed as an alternative T2-weighted contrast agent, with the goal of further reducing the required injection concentration through utilization of superparamagnetism of the iron-core. Treatments were performed with blood clots produced from human blood to mimic the composition of a clot in a stroke model, while fluid blood was used as the pool to represent blood within vessels. T2-weighted scans, using a $7 \mathrm{~T}$ MRI scanner, were conducted to assess whether treatment with $\mathrm{FA}-\mathrm{Fe}_{3} \mathrm{O}_{4}$ AuNPs would result in specific accumulation of the contrast at the location of the clot and thereby produce significant and visually observable contrast enhancement. Contrast 
enhancement achieved in incubations of $\mathrm{FA}-\mathrm{Fe}_{3} \mathrm{O}_{4}$-AuNPs with blood clots suspended in blood pool or PBS was compared to the control treatments of blood clots with FB139$\mathrm{Fe}_{3} \mathrm{O}_{4}-\mathrm{AuNPs}$ and PBS. All measurements were performed after a 45-minute incubation to allow for equilibration of the interaction of the aptamer with the fibrin target. FB139 was chosen as the control sequence, as it is similar in length and characteristics, but was originally selected for a different target (toxin). ${ }^{169}$ The workflow of the sample preparation is outlined in Figure 5.1A. Visual assessment of the T2-weighted images confirmed that functionalization with FA was crucial for specific accumulation of $\mathrm{Fe}_{3} \mathrm{O}_{4}-\mathrm{AuNPs}$ on the surface of the blood clots. The clots that were treated with $\mathrm{FA}-\mathrm{Fe}_{3} \mathrm{O}_{4}-\mathrm{AuNPs}$ were highly resolved, due to the presence of hypointense contrast throughout the circumference of the

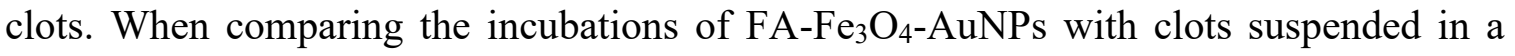
blood pool and those in PBS, the contrast enhancement is much more pronounced visually in the latter (Figure 5.1B). This is likely related to the presence of fibrinogen within the blood pool. Fibrinogen, being one of the natural components of whole blood, would be present at concentrations of $160-400 \mathrm{mg} / \mathrm{dL} .{ }^{195}$ As previously mentioned, FA was originally selected to bind human fibrinogen. ${ }^{28}$ Any binding of $\mathrm{FA}^{2}-\mathrm{Fe}_{3} \mathrm{O}_{4}-\mathrm{AuNPs}$ to fibrinogen in the blood pool may effectively decrease the number of available nanoparticles related to the injected concentration. While this binding is possible, it does not significantly decrease the measured contrast at the location of the clots (Figure 5.1C). Overall, FAdirected co-localization of $\mathrm{Fe}_{3} \mathrm{O}_{4}$-AuNPs results in high local concentrations of the contrast material and pronounced negative $\mathrm{T} 2$ contrast. When comparing to the control treatments, no observable clot delineation was found. Even though $\mathrm{FB} 139-\mathrm{Fe}_{3} \mathrm{O}_{4}-\mathrm{AuNPs}$ were present within the blood pool, the lack of target binding-associated co-localization resulted in 
overall diluted contrast material, the concentrations of which were not high enough to be observed. In fact, the images for incubations with $\mathrm{FB} 139-\mathrm{Fe}_{3} \mathrm{O}_{4}-\mathrm{AuNPs}$ were the same as incubations without the contrast present (in PBS) (Figure 5.1B).

In order to confirm if the visually observed trends are significant, contrast was measured based on the average pixel intensities (API) of each of the samples. The treatments were compared using One-way ANOVA test $(\mathrm{F}(3,20)=29.08, \mathrm{p}<0.0001$ at $\alpha=$ $0.05)$ indicating that there is a statistically significant difference between one or more. Posthoc Tukey HSD test confirmed that targeted treatments were significantly different from untargeted treatments $(p<0.01)$. Specifically, while contrast produced in incubations of the clots with $\mathrm{FA}-\mathrm{Fe}_{3} \mathrm{O}_{4}-\mathrm{AuNPs}$, in the presence of the blood pool and PBS, were not significantly different from one another $(\mathrm{p}=0.90)$, both were significantly different from the control incubations with $\mathrm{FB} 139-\mathrm{Fe}_{3} \mathrm{O}_{4}-\mathrm{AuNPs}(\mathrm{p}<0.01)$. The same was true when those treatments were compared to incubations with only PBS present $(\mathrm{p}<0.01)$. To further reaffirm the specificity of this contrast material, thereby confirming that contrast

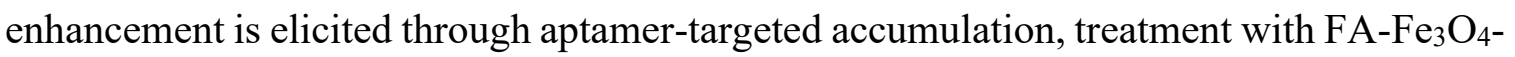
AuNPs and PBS were compared, demonstrating no significant difference $(\mathrm{p}=0.47)$. This analysis confirms that $\mathrm{FA}-\mathrm{Fe}_{3} \mathrm{O}_{4}-\mathrm{AuNPs}$ can be employed as targeted contrast material for imaging of blood clots, resulting in highly defined clot parameters. The average contrast measurements for each treatment are summarized in Table 5.1. 
Table 5.1: Average contrast of blood clots associated with each treatment after a 45-minute incubation, measured in T2-weighted MRI.

\begin{tabular}{|l|l|l|l|}
\hline $\begin{array}{l}\text { FA-Fe3O4-AuNPs } \\
\text { + blood clot }+ \\
\text { blood pool }\end{array}$ & $\begin{array}{l}\text { FA-Fe3O4-AuNPs } \\
\text { + blood clot + PBS }\end{array}$ & $\begin{array}{l}\text { FB139-Fe3O4- } \\
\text { AuNPs + blood clot } \\
+ \text { blood pool }\end{array}$ & $\begin{array}{l}\text { PBS + blood clot + } \\
\text { blood pool }\end{array}$ \\
\hline $546.3( \pm 48.4)$ & $551.6( \pm 80.6)$ & $810.4( \pm 79.8)$ & $757.2( \pm 18.9)$ \\
\hline
\end{tabular}

A

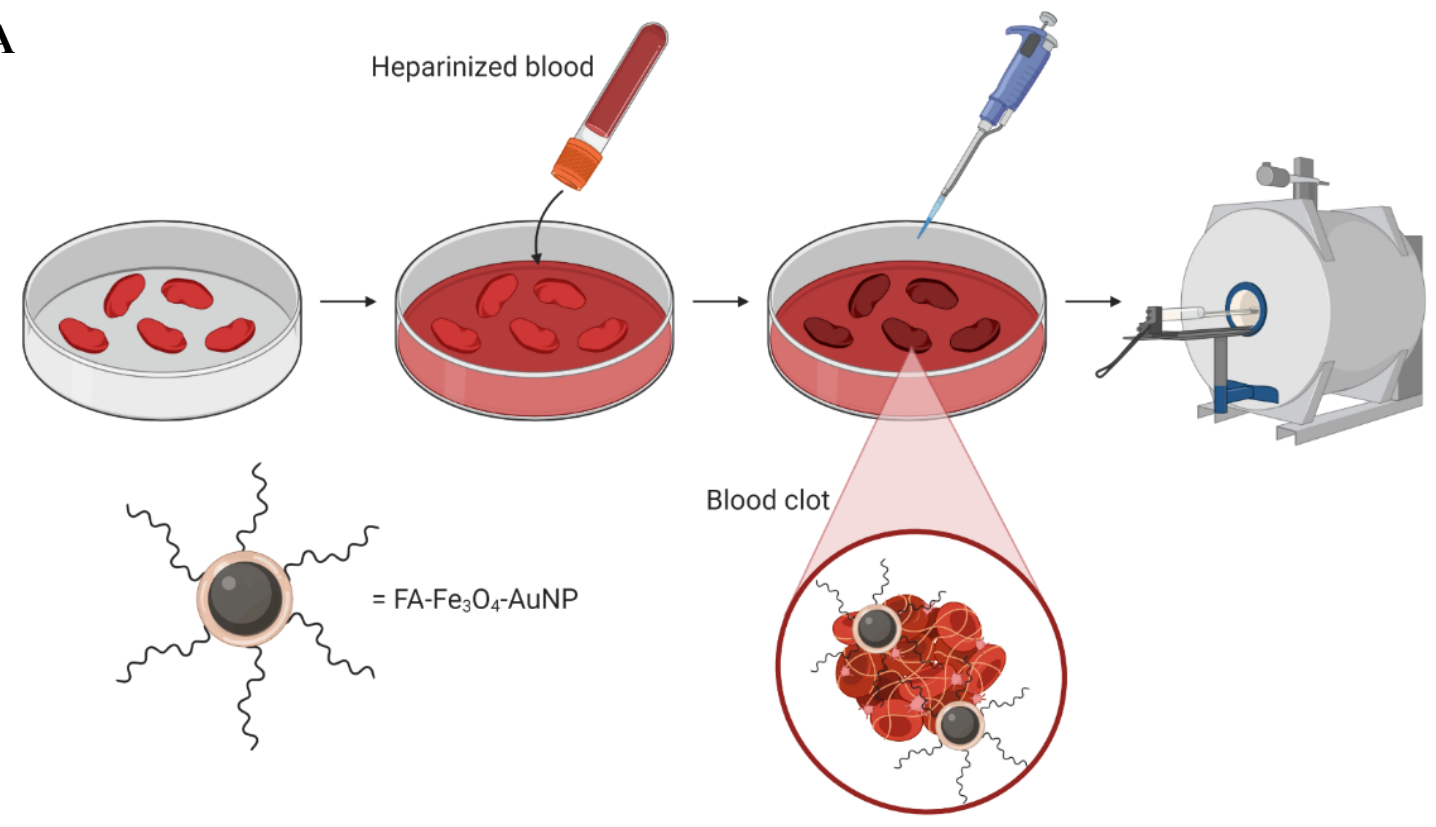

B

T2-weighted MRI (triplicate samples)

$\mathrm{FA}_{\mathrm{Fe}} \mathrm{O}_{4}$-AuNPs + blood clot + blood pool

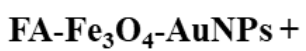
blood clot + PBS

FB139- $\mathrm{Fe}_{3} \mathrm{O}_{4}$-AuNPs + blood clot + blood pool

PBS + blood clot + blood pool

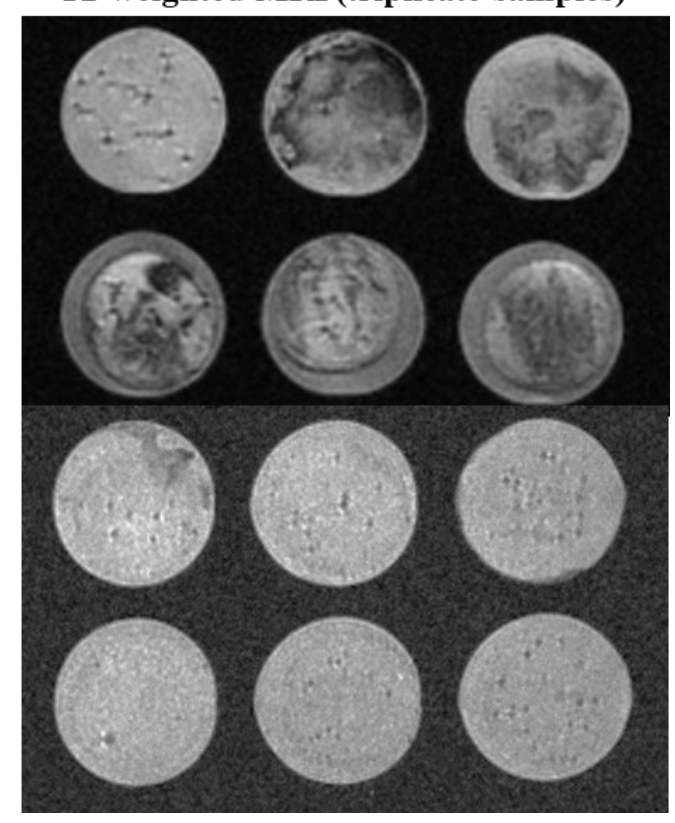


C

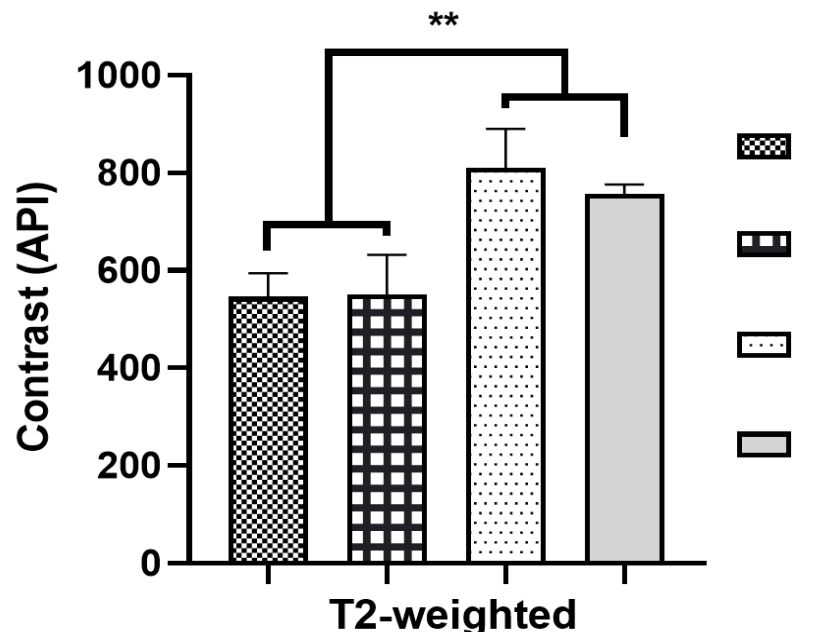

$\mathrm{FA}-\mathrm{Fe}_{3} \mathrm{O}_{4}-\mathrm{AuNPs}+$ blood clot + blood pool

$\mathrm{FA}-\mathrm{Fe}_{3} \mathrm{O}_{4}$-AuNPs + blood clot + PBS $\mathrm{FB139}-\mathrm{Fe}_{3} \mathrm{O}_{4}-\mathrm{AuNPs}+$ blood clot + blood pool PBS + blood clot + blood pool

Figure 5.1: (A) Schematic representation of sample preparation for in vitro MRI imaging, made in BioRender; (B) T2-weighted MR images of clotted blood (done in triplicate) that underwent the following treatments: $1^{\text {st }}$ row - clotted blood in a heparinized blood pool treated with $\mathrm{FA}_{-}-\mathrm{Fe}_{3} \mathrm{O}_{4}-$ AuNPs, $2^{\text {nd }}$ row - clotted blood in a PBS pool treated with FA-Fe3 $\mathrm{O}_{4}-\mathrm{AuNPs}, 3^{\text {rd }}$ row - clotted blood in

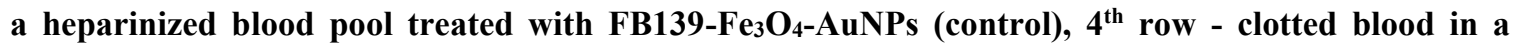
heparinized blood pool treated with PBS (control); (D) Average contrast enhancement of each sample, measured by ImageJ Software and compared using One-way ANOVA test $(F(3,20)=29.08, p<0.0001$ at $\alpha=0.05)$; Tukey post-hoc analyses revealed a significant difference between treatments $(* * p<0.01)$.

\subsubsection{Phantom and in vitro CT imaging (human blood)}

Based on the presence of the gold coating, we also sought to assess whether FA$\mathrm{Fe}_{3} \mathrm{O}_{4}$-AuNPs can be used as a CT contrast agent. AuNPs have been researched for applications in CT imaging, due to many favourable characteristics of gold, including its ability exhibit higher X-ray attenuation than iodine. ${ }^{252}$ With that in mind, $\mathrm{FA}-\mathrm{Fe}_{3} \mathrm{O}_{4}$ AuNPs were tested for their capability to produce measurable CT contrast. Concentrations achievable during the synthesis were considerably lower than that of Isovue, which was used as a control. Nevertheless, increasing concentrations of FA-Fe $\mathrm{O}_{4}-\mathrm{AuNPs}$ resulted in a linear increase of measured contrast (HU) (Figure 5.2). The slope of the linear 
relationship between the contrast and the concentration was measured to be $1697 \mathrm{HU} \mu \mathrm{M}^{-}$

1. This is considerably higher than that of AuNPs tested in Chapter 4.

A
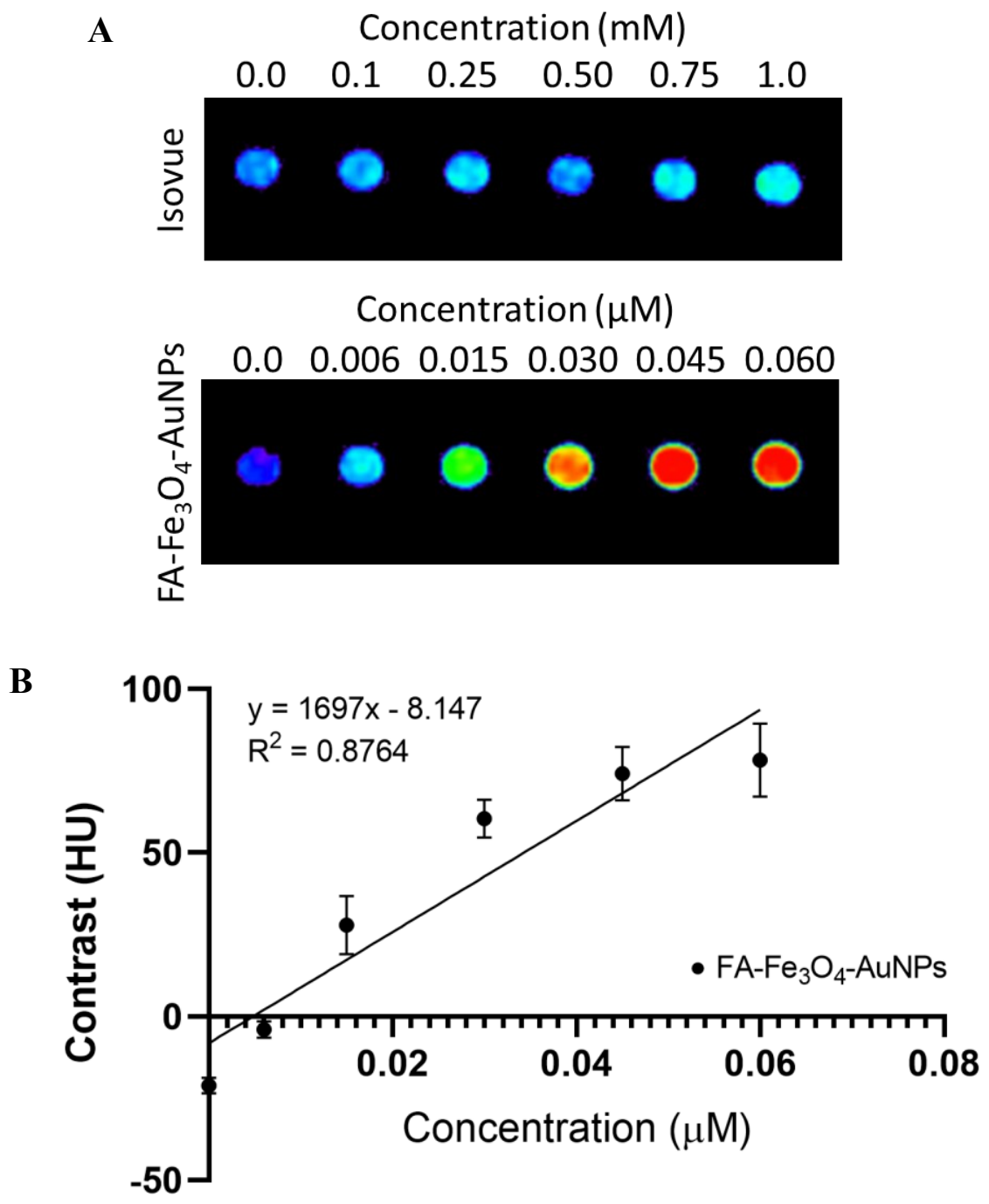

Figure 5.2: (A) CT contrast phantom images of Isovue (iopamidol) and $\mathrm{FA}-\mathrm{FA}-\mathrm{Fe}_{3} \mathrm{O}_{4}-\mathrm{AuNPs}_{\text {, both }}$ with increasing concentrations, at $100 \mathrm{kVp}$; (B) Plot of concentration-dependent contrast enhancement (HU), measured with software provided with the CT scanner, for $\mathrm{FA}_{-} \mathrm{Fe}_{3} \mathrm{O}_{4}-\mathrm{AuNPs}$.

Subsequently, $\mathrm{FA}-\mathrm{Fe}_{3} \mathrm{O}_{4}$-AuNPs were tested in vitro to verify that target-specific contrast enhancement is observed. All samples were prepared in the same manner as for MRI, with control samples including clinically available CT contrast agent, Isovue, FB139- 
$\mathrm{Fe}_{3} \mathrm{O}_{4}$-AuNPs, and PBS. Unfortunately, no observable or statistical difference was detected when comparing treatments with $\mathrm{FA}-\mathrm{Fe}_{3} \mathrm{O}_{4}-\mathrm{AuNPs}$ to those with $\mathrm{FB} 139-\mathrm{Fe}_{3} \mathrm{O}_{4}-\mathrm{AuNPs}$, Isovue, and PBS. We can infer that the gold coating was not sufficient enough at $60 \mathrm{nM}$ concentrations to produce CT contrast and likely would need to be much thicker to be successful. Therefore, the susceptibility of $\mathrm{FA}-\mathrm{Fe}_{3} \mathrm{O}_{4}-\mathrm{AuNPs}$ to dilution in the final volume of the samples was considerable (Figure S5.6).

To the best of our knowledge, this is the first fibrin-targeting contrast material synthesized using CSNs, specifically comprising of an iron-oxide core and gold-coating. As previously demonstrated by our group, we were able to use a formerly selected aptamer which binds monomeric fibrinogen. ${ }^{215}$ This streamlined the development of fibrin-specific MRI contrast material. In addition, validation of this method could also open doors for the discovery of other aptamers which are selected using monomeric proteins, specifically in situations where the polymeric form is insoluble. An important consideration for implementation of this type of selection would be whether the structural elements of the monomer remain largely unchanged when polymerized. In the context of this novel contrast material, it was designed to increase stability and ease of functionalization through the incorporation of gold coating, provide targeting capabilities through surfacemodification with FA, and possess superparamagnetic properties through the composition

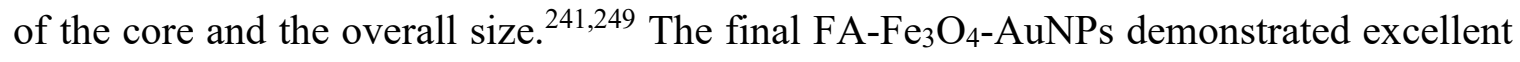
dispersion in TEM and was easily functionalized for fibrin targeting. The size of the nanoparticles was appropriate to experience superparamagnetism and serve as T2enhancing contrast agent. ${ }^{239}$ Even at low nanomolar concentrations, FA- $\mathrm{Fe}_{3} \mathrm{O}_{4}$-AuNPs were able to produce significant contrast enhancement that was readily visualized in in 
vitro T2-weighted MRI. This was expected, as FA is able to target the fibrin clot, thereby increasing the local concentration of $\mathrm{FA}-\mathrm{Fe}_{3} \mathrm{O}_{4}-\mathrm{AuNPs}$ and enhancing the magnetic effect of the $\mathrm{Fe}_{3} \mathrm{O}_{4}$-core on surrounding protons. Ligand-binding was important for observable contrast to be developed, allowing for lower overall concentrations to be used upon injection of contrast media. Although $\mathrm{Fe}_{3} \mathrm{O}_{4}$-AuNP-associated contrast is hypointense in T2-weighted imaging and could be considered unfavourable as a "turn off" response, it was target-specific and intense enough to observe. This was especially highlighted in highresolution T2-weighted imaging (acquired over several hours), though this type of imaging is not clinically relevant and was not discussed. (Figure S5.7). It is clear that $\mathrm{Fe}_{3} \mathrm{O}_{4}-\mathrm{AuNPs}$ accumulated specifically on surface of the clot, binding accessible fibrinogen monomer structures.

Ligand-targeting in the development of fibrin-specific contrast media has been previously explored, as outlined by Katie Ciesienski. ${ }^{253}$ Most commonly, fibrin is targeted by a short peptide, EP-2104R, which has been used in formulations with Gd-based contrast media. Fibrin-targeting has also been achieved through the use of anti-fibrin antibodies, conjugated to the surface of manganese-based nanoparticles. ${ }^{196,253,254}$ With the help of these molecular recognition elements, fibrin can be detected by direct accumulation of contrast at its location. This is advantageous in many scenarios, particularly as it pertains to stroke imaging. That is, thrombi are currently identified through looking for a void in contrast flow, which drastically limits diagnostic capabilities. In addition, characterization of thrombi is very restricted, typically not allowing to gather information such as fibrin content. ${ }^{139,253}$ Our aptamer-targeted formulation would allow for such assessment, with contrast accumulation related to the presence of fibrin. Moreover, it would allow for use of 
lower concentrations, limiting potential undesirable site-effects associated with current clinical contrast agents. ${ }^{53}$ Meanwhile, the gold coating would protect the iron-oxide core increasing stability and lowering toxicity in biological systems. ${ }^{255}$ If this formulation is successful, it could eventually lead to its utilization in whole-body MRI, simplifying diagnosis of conditions that involve clotting, as well as monitoring their follow-up. 


\subsection{Supporting information}

\subsubsection{Additional methods}

\subsubsection{Assessment of magnetic properties of $\mathrm{FA}-\mathrm{Fe}_{3} \mathrm{O}_{4}-\mathrm{AuNPs}$}

To evaluate if surface functionalization of $\mathrm{Fe}_{3} \mathrm{O}_{4}$-AuNPs with $\mathrm{FA}$ and the subsequent binding of $\mathrm{FA}-\mathrm{Fe}_{3} \mathrm{O}_{4}-\mathrm{AuNPs}$ to fibrin perturbs the magnetic properties, $100 \mu \mathrm{L}$ of $100 \mathrm{nM} \mathrm{FA}-\mathrm{Fe}_{3} \mathrm{O}_{4}$-AuNPs was incubated with $0.005 \mathrm{~g}$ of lyophilized fibrin from human plasma for 1 hour. As a control, fibrin was incubated with bare $\mathrm{Fe}_{3} \mathrm{O}_{4}$-AuNPs. After incubation, all samples were washed 10x with PBS buffer, $\mathrm{pH} 7.4$ to wash away unbound nanoparticles. Rare earth magnet was used to assess whether FA-Fe $\mathrm{O}_{4}$-AuNPs-bound fibrin would exhibit magnetic properties.

\subsubsection{Additional results and discussion}

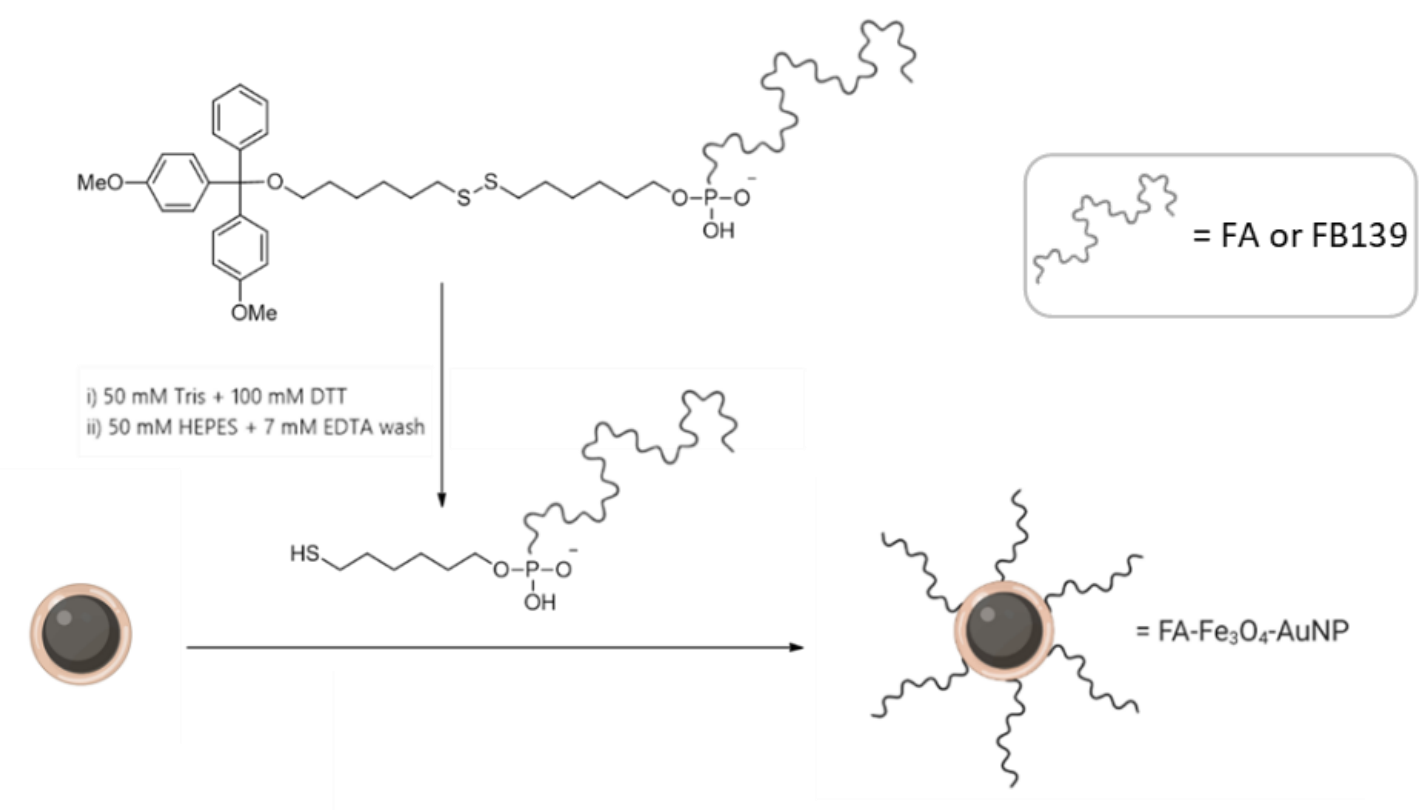

Scheme S5.1: Reaction scheme outlining the cleavage of the disulfide bond to liberate a free thiol group and its subsequent conjugation to the surface of $\mathrm{Fe}_{3} \mathrm{O}_{4}-\mathrm{AuNPs}$, using the gold shell 
A

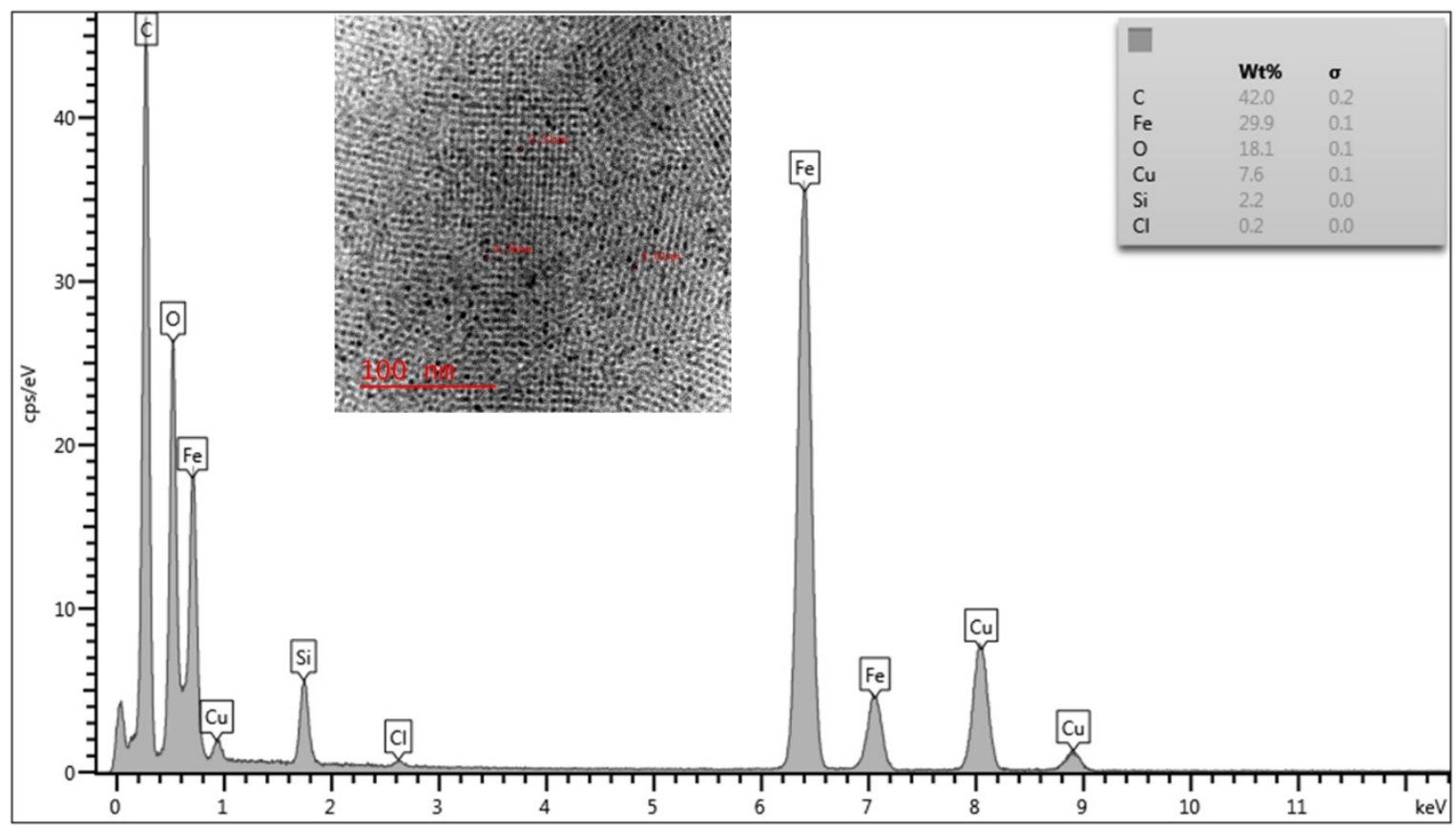

B

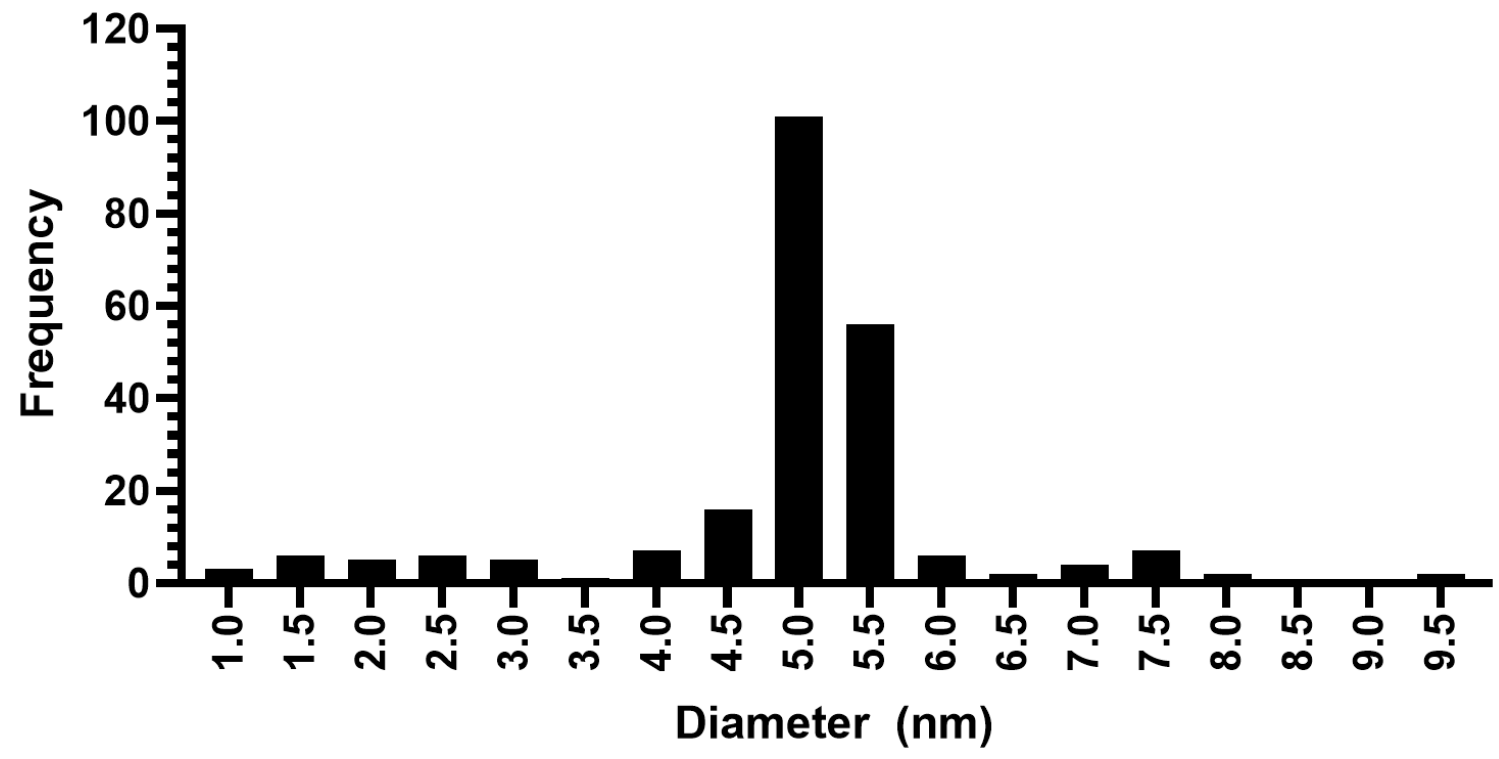

Figure S5.1: (A) Transmission electron microscopy (TEM) image with $100 \mathrm{~nm}$ scale bar and corresponding energy-dispersive $\mathrm{X}$-ray spectroscopy (EDS) spectrum of small, monodispersed $\mathrm{Fe}_{3} \mathrm{O}_{4}$ core. Strong EDS peaks for both Fe and $O$ indicate the presence of iron oxide. (B) Histogram demonstrating the size distribution, with average diameter of $4.95 \pm 1.27(n=229)$. 
A
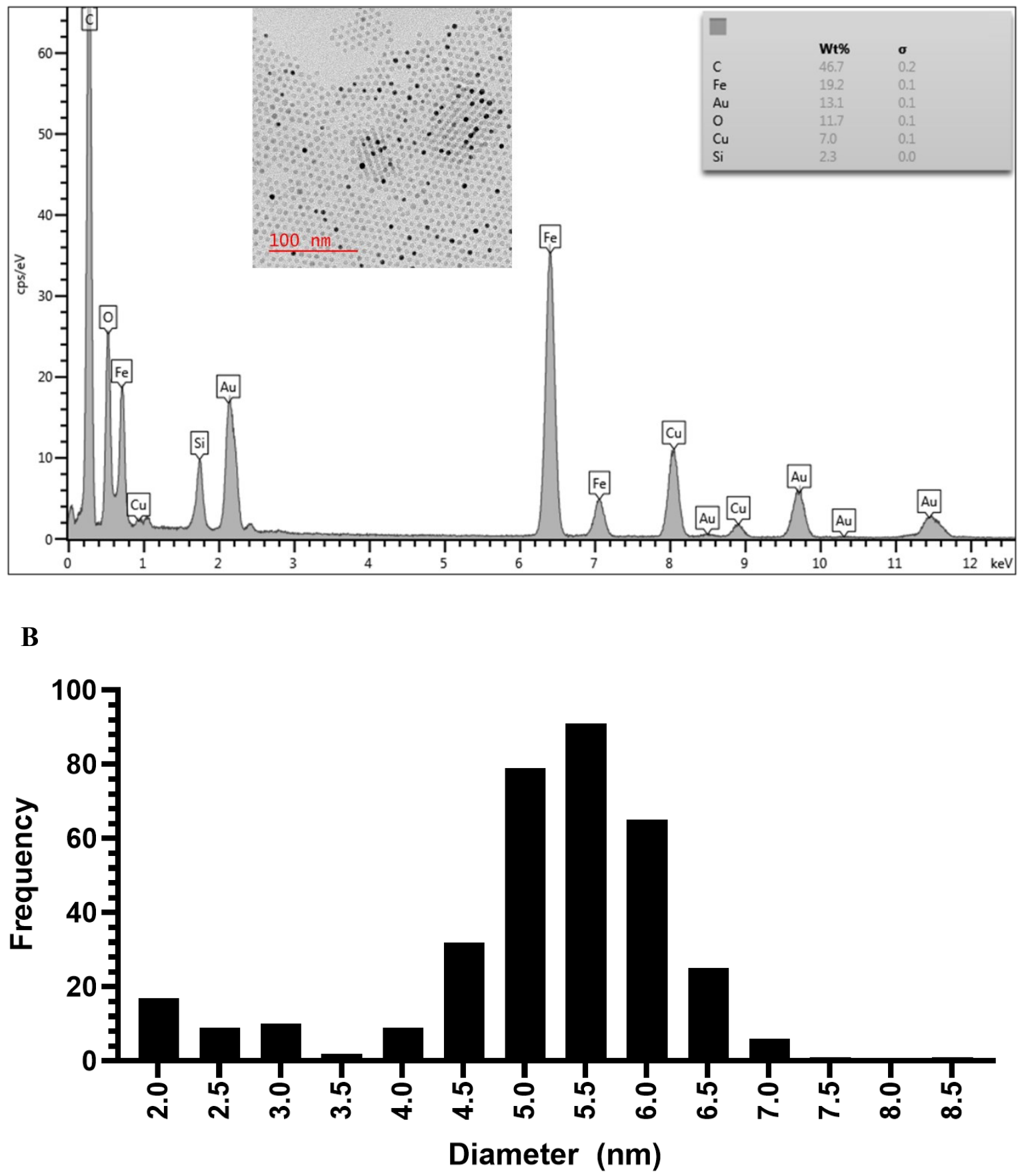

Figure S5.2: (A) TEM image with $100 \mathrm{~nm}$ scale bar and corresponding EDS spectrum of the $\mathrm{Fe}_{3} \mathrm{O}_{4}$ AuNPs in phenyl ether. Strong EDS peaks for both Fe and $\mathrm{O}$ indicate the presence of iron oxide, while the presence of a peak for Au confirms gold-coating. (B) Histogram demonstrating the size distribution, with average diameter of $5.13 \pm 1.14(n=347)$. 
$\mathbf{A}$

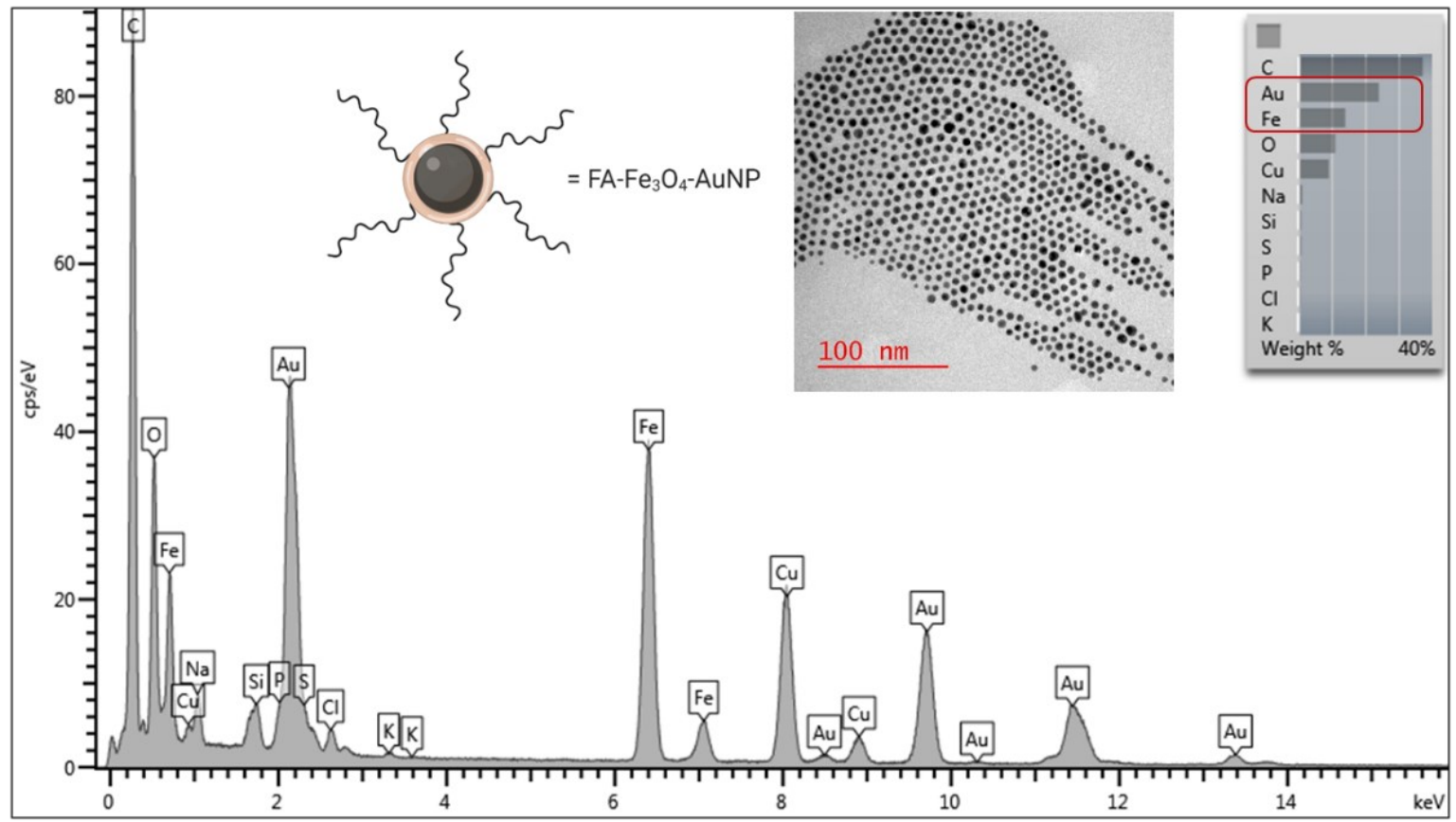

B

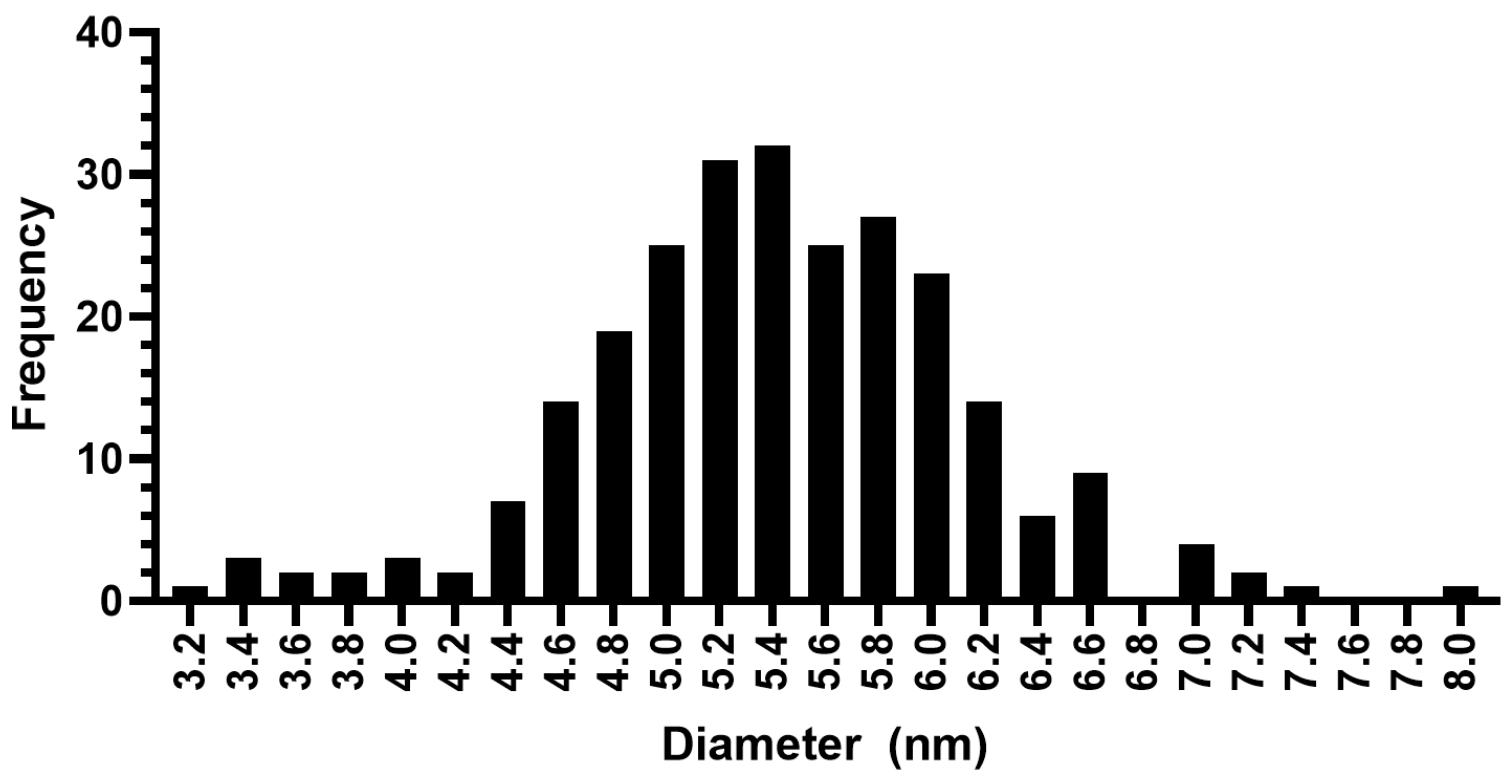

Figure S5.3: TEM image with $100 \mathrm{~nm}$ scale bar and corresponding EDS spectrum of $\mathrm{FA}^{-\mathrm{Fe}_{3} \mathrm{O}_{4}-\mathrm{AuNPs}}$ in $\operatorname{diH}_{2} \mathrm{O}$. (B) Histogram demonstrating the size distribution, with average diameter of $5.42 \pm 0.72$ $(\mathbf{n}=\mathbf{2 5 3})$. 

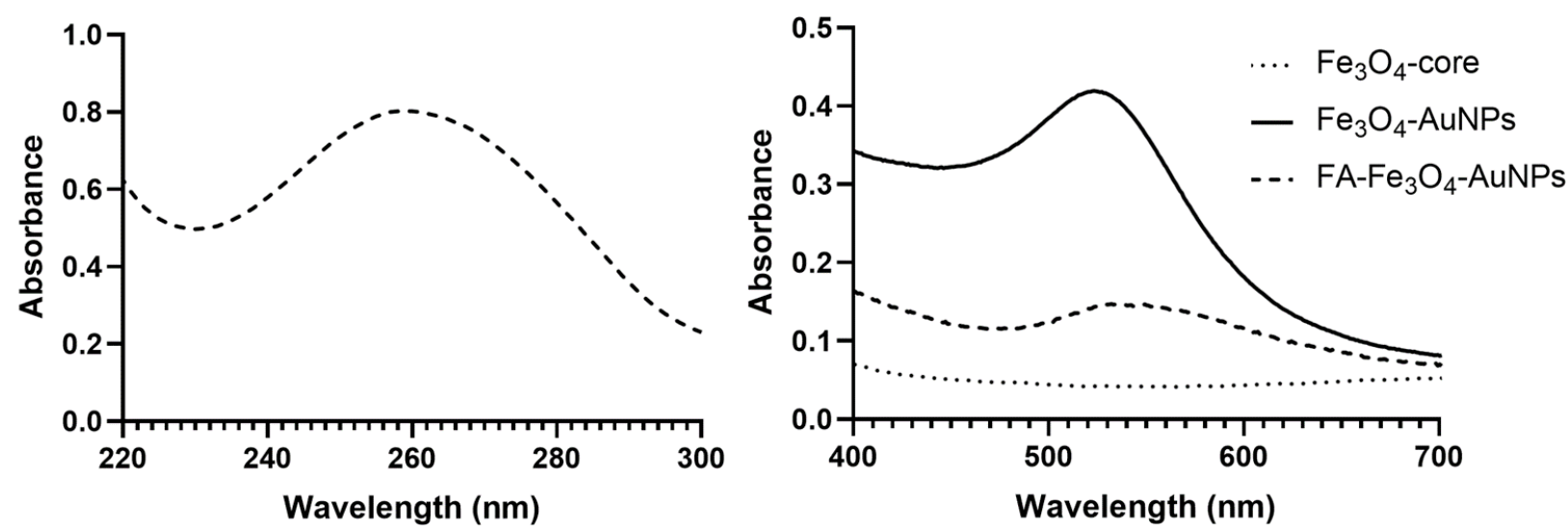

Figure S5.4: UV-Vis spectra of $\mathrm{Fe}_{3} \mathrm{O}_{4}-\mathrm{core}, \mathrm{Fe}_{3} \mathrm{O}_{4}-\mathrm{AuNPs}$, and $\mathrm{FA}-\mathrm{Fe}_{3} \mathrm{O}_{4}-\mathrm{AuNPs}$ in their respective buffers

The ability of FA to bind fibrin has previously been confirmed. ${ }^{215}$ In this formulation, it was important that such binding does not lead to loss of magnetic properties, as they are crucial to MRI. Incubation with $\mathrm{FA}-\mathrm{Fe}_{3} \mathrm{O}_{4}$-AuNPs allowed for binding to occur, as evident by the presence of dark colour, associated with $\mathrm{FA}-\mathrm{Fe}_{3} \mathrm{O}_{4}-\mathrm{AuNPs}$ bound on the surface of fibrin (Figure S5.5 - middle). This was not observed when fibrin was treated with bare $\mathrm{Fe}_{3} \mathrm{O}_{4}$-AuNPs, which also confirmed that PBS washes likely got rid of the majority of non-targeted nanoparticles (Figure S5.5 - right). Placing FA-Fe $\mathrm{O}_{4}$-AuNPs treated fibrin on a magnet resulted in accumulation of the smaller sized particles in the middle, indicating that $\mathrm{FA}-\mathrm{Fe}_{3} \mathrm{O}_{4}-\mathrm{AuNPs}$ retains its magnetic properties when bound to the target (Figure S5.5 - left). 


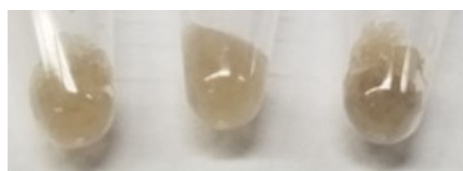

Fibrin + $\mathrm{Fe}_{3} \mathrm{O}_{4}$-AuNPs

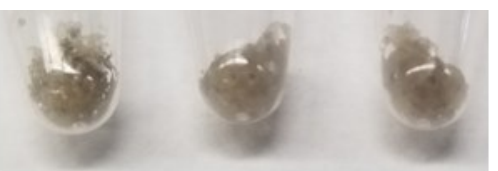

Fibrin +

$\mathrm{FA}-\mathrm{Fe}_{3} \mathrm{O}_{4}$-AuNPs

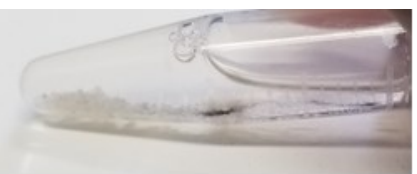

Fibrin +

$\mathrm{FA}-\mathrm{Fe}_{3} \mathrm{O}_{4}$-AuNPs on a magnet

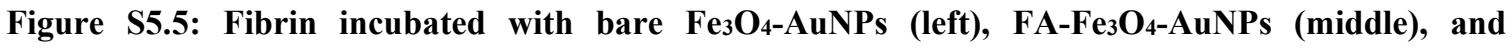
magnetized fibrin (with bound $\mathrm{FA}-\mathrm{Fe}_{3} \mathrm{O}_{4}$-AuNPs after 10x wash with $\mathrm{PBS}$ ) on a rare earth magnet. Fibrin magnetization is demonstrated by accumulation of small dark powder accumulating in the middle. 
A

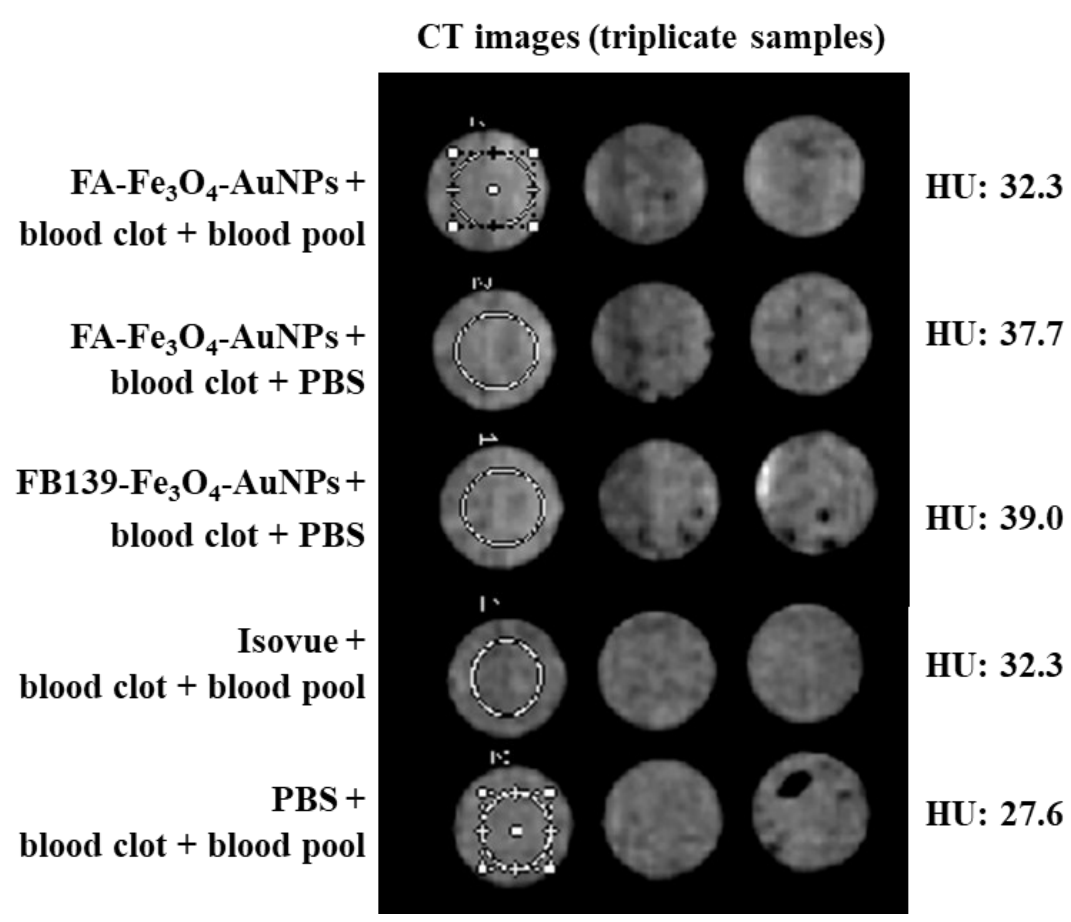

B

CT images

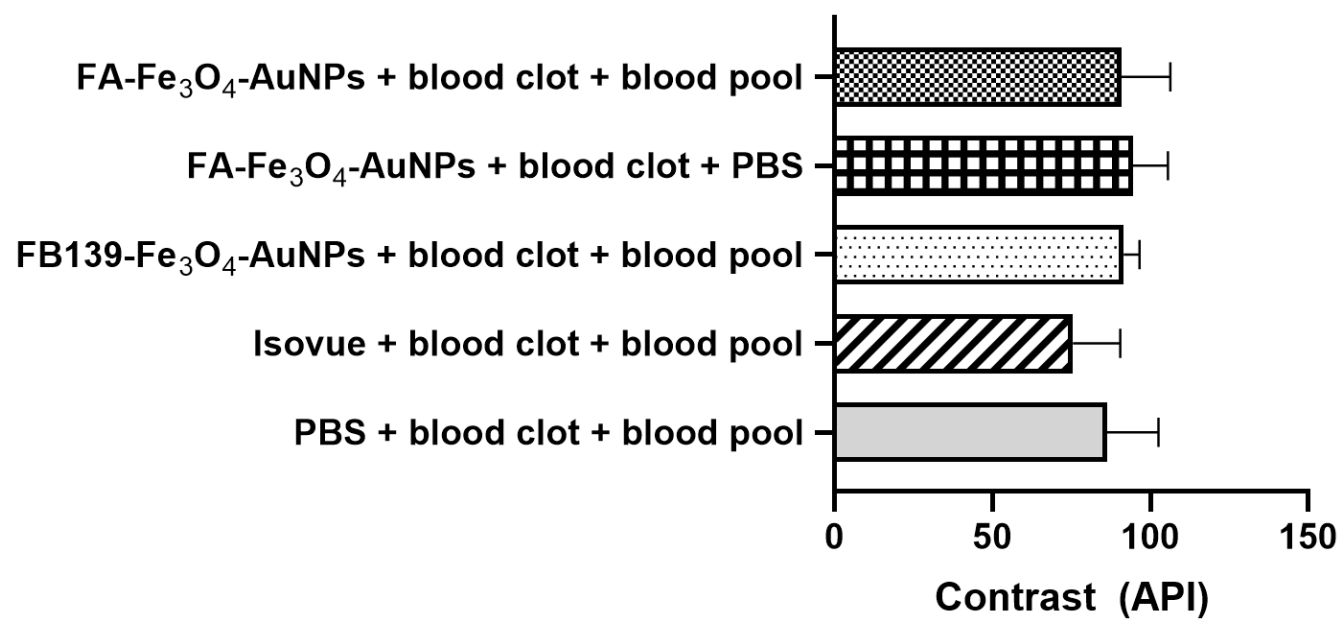

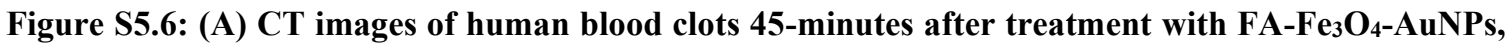

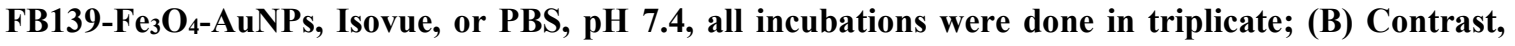
measured based on average pixel intensity using ImageJ software, associated with each treatment. 


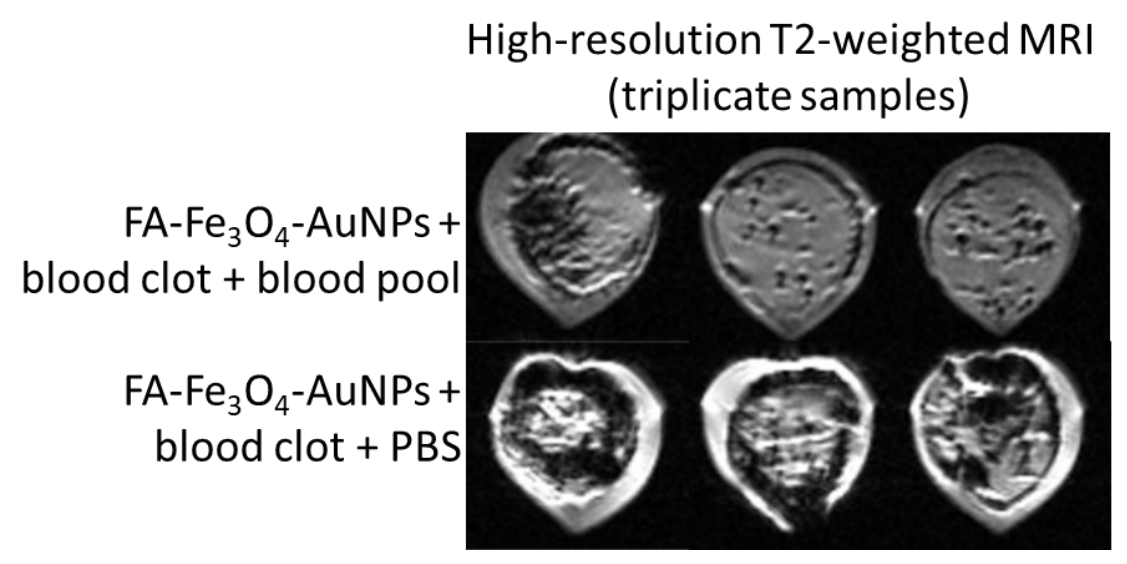

Figure S5.7: High-resolution T2-weighted images of blood clots that underwent the following

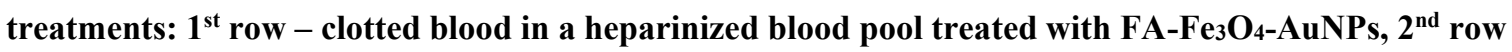
- clotted blood in a PBS pool treated with $\mathrm{FA}^{-\mathrm{Fe}_{3} \mathrm{O}_{4}-\mathrm{AuNPs}}$ 


\section{Chapter 6: Conclusions and future directions}

\subsection{Overall conclusions}

Vascular diseases represent a large public health burden and, therefore, land themselves at the forefront of research towards more effective diagnosis and treatments. $^{38,42,44,45}$ The research outlined in this thesis centered around two types of manifestations related to the presence of blood clots. Specifically, clots that are spontaneously formed as a result of hemostatic malfunctions, leading to ischemic strokes, and clots that are formed in an effort to seal the site of a hemorrhage in a hemorrhagic stroke. In both cases, it has been identified that full delineation of the clot pathology would streamline diagnosis and treatment of the aforementioned conditions. ${ }^{34-37}$ Since current imaging modalities are only able to pinpoint the general location of a vascular occlusion based on the overall flow (or absence thereof) of contrast, essentially diagnosing by exclusion, molecular imaging of blood clot constituents has been described as an excellent alternative. ${ }^{31,48,53,154,160,165}$ In addition, clinically available contrast agents for both MRI and CT imaging are known to cause a number of undesirable side-effects, and require high injection concentrations to produce contrast enhancement as they are distributed throughout the entirety of the vascular system. ${ }^{53,55,57,58}$

To lower the toxicity and increase the specificity, novel contrast formulations were produced for both MRI and CT. We sought to incorporate targeting elements into materials that are known to achieve measurable contrast enhancement in biological systems. Overall, four novel aptamer-targeted materials were synthesized and characterized. We were able to successfully streamline the production of these conjugates by using an aptamer that was previously selected to bind the fibrinogen monomer. Through a number of binding 
validation studies, it became evident that FA was able to bind the polymeric protein successfully and selectively. This proof-of-concept method opens doors for the development of other aptamer-targeted materials, given an aptamer that is selected for the monomeric form which remains predominantly unchanged when polymerized. That is, polymer targets may be difficult to select against, especially when insoluble, and selection against the monomeric form that is influenced towards a known region would make such elucidation more accessible.

All novel formulations proved to be capable of producing contrast enhancement that was more pronounced than the clinically available agents, even in the absence of target binding. This effect was further intensified in the presence of fibrin, with aptamer-targeting resulting in co-localization of the contrast formulations, leading to high local concentrations. In MRI, binding of Gd(III)-NOTA-FA to the fibrous clot resulted in conversion to T2-weighted enhancement, which can be used to increase specificity and highlight the location of the clot. In CT, when blood clots were treated with iodo-FA and FA-AuNPs, fibrin targeting allowed for use of millimolar and micromolar concentrations, respectively. This is orders of magnitude lower than the concentrations at which clinical contrast agents, such as iopamidol, are administered. Finally, the dual-contrast agent, FA$\mathrm{Fe}_{3} \mathrm{O}_{4}$-AuNPs, proved to be an excellent T2-weighted alternative in MRI imaging of blood clots, producing well resolved images through delineation of the clot surface. Unfortunately, the presence of the gold surface was not sufficient to produce observable CT contrast. Nevertheless, gold was used to allow for easy surface-functionalization with FA. Importantly, the presence, and presumed binding of FA with blood fibrinogen, did not disrupt the clotting cascade, ensuring that these aptamer-contrast formulations will not act 
as a thrombolytic. The overall characteristics of these novel formulations, as well as their strengths and weaknesses are summarized in Table 6.1.

Table 6.1: Summary of novel conjugates produced in this research, as well as their strengths and weaknesses identified at this time

\begin{tabular}{|c|c|c|c|c|c|}
\hline & $\begin{array}{l}\text { Aptamer- } \\
\text { targeted } \\
\text { contrast }\end{array}$ & $\begin{array}{l}\text { Contrast } \\
\text { enhancement } \\
\text { (clinical } \\
\text { scanners) }\end{array}$ & $\begin{array}{l}\text { Blood } \\
\text { clot } \\
\text { binding }\end{array}$ & Advantages & $\begin{array}{l}\text { Dis- } \\
\text { advantages }\end{array}$ \\
\hline$M R I$ & $\begin{array}{l}\text { Gd(III)- } \\
\text { NOTA- } \\
\text { FA }\end{array}$ & $\begin{array}{l}\mathrm{R} 1: 3.04 \mathrm{mM}^{-1} \mathrm{~s}^{-1} \\
\mathrm{R} 2: 38.7 \mathrm{mM}^{-1} \mathrm{~s}^{-1}\end{array}$ & $\begin{array}{l}\text { Interior } \\
\text { network }\end{array}$ & $\begin{array}{l}\text { High-resolution in T2- } \\
\text { weighted imaging }\end{array}$ & $\begin{array}{l}\text { May de- } \\
\text { chelate toxic } \\
\text { Gd(III) ion }\end{array}$ \\
\hline \multirow[t]{2}{*}{$C T$} & Iodo-FA & $40.5 \mathrm{HU} \mathrm{mM}^{-1}$ & $\begin{array}{l}\text { Interior } \\
\text { network }\end{array}$ & Higher iodine content & $\begin{array}{l}\text { Harder to } \\
\text { achieve high- } \\
\text { resolution } \\
\text { (fibrinogen } \\
\text { binding) }\end{array}$ \\
\hline & FA-AuNP & $563.6 \mathrm{HU} \mu \mathrm{M}^{-1}$ & $\begin{array}{l}\text { Surface- } \\
\text { binding }\end{array}$ & $\begin{array}{l}\text { Alternative material to } \\
\text { avoid allergic reaction }\end{array}$ & $\begin{array}{l}\text { Does not enter } \\
\text { blood clots }\end{array}$ \\
\hline $\begin{array}{l}\text { Dual } \\
\text { agent }\end{array}$ & $\begin{array}{l}\text { FA- } \\
\mathrm{Fe}_{3} \mathrm{O}_{4-} \\
\mathrm{AuNP}\end{array}$ & $\begin{array}{l}\text { MRI: N/A } \\
\text { CT: } 1697 \mathrm{HU} \\
\mu \mathrm{M}^{-1}\end{array}$ & $\begin{array}{l}\text { Interior } \\
\text { network }\end{array}$ & $\begin{array}{l}\text { MRI: high resolution in } \\
\text { T2-weighted imaging } \\
\mathrm{CT} \text { : alternative material } \\
\text { to avoid allergic } \\
\text { reaction } \\
\text { Both: single contrast } \\
\text { agent for two } \\
\text { modalities }\end{array}$ & $\begin{array}{l}\text { Extensive } \\
\text { synthesis with } \\
\text { insufficient } \\
\text { gold coating }\end{array}$ \\
\hline
\end{tabular}

Clinically available contrast agents have recently come under scrutiny for their potential toxicity. Specifically, gadolinium-based contrast agents are associated with acute nephrogenic systemic fibrosis, as well as brain, kidney, and bone tissue accumulation. ${ }^{53}$ Meanwhile, iodinated contrast media induce acute kidney injury and contrast-induced nephropathy. In addition, a number of patients experience moderate-to-severe allergic 
reactions to iodinated small molecules. ${ }^{58,227}$ The aptamer-targeted contrast formulations outlined in this thesis were designed to decrease the potential chemical toxicity. By increasing the overall ability to produce contrast enhancement, each of these novel formulations proved to be effective at considerably lower concentrations when compared to their clinically available counterparts. Aptamer-targeting resulted in local accumulation of the contrast material, providing high resolution images, thereby minimizing repeated administration. Alternative materials which exhibit minimal toxicity were produced to provide an alternative to patients that are particularly susceptible to toxic effects of gadolinium- and iodine-based agents. Based on their size, it is predicted that aptamertargeted gadolinium conjugates and iodinated aptamers will undergo renal clearance. On the other hand, nanoparticle-based formulations, including aptamer-functionalized gold nanoparticles and core-shell nanoparticles will likely undergo hepatic clearance. By increasing the overall size of the contrast formulation, the clearance route may be rerouted, which is particularly advantageous in patients with existing kidney issues. ${ }^{256}$

While proven to be successful in these proof-of-concept studies, it is important to recognize the limitations of these formulations. One of the drawbacks of using a previously selected aptamer is that, in the presence of fibrinogen within the biological system, a proportion of the FA-targeted contrast will bind fibrinogen in the blood pool. ${ }^{26}$ When blood clots were tested in the presence of a fluid blood pool in vitro, this binding had to be accounted for. When translated to in vivo testing, this limited fibrin targeting to such degree that the blood clots could not be well resolved. Moreover, since no stabilizing modifications were incorporated into the final formulations, it was not clear whether the aptamer formulations were affected by nucleases. In addition, the overall cost of the 
production of aptamer-targeted materials is higher than that of the currently utilized contrast agents. When opting for the use of these novel materials, the cost-to-benefit ratio must be considered.

\subsection{Future directions}

Given the success of peptide-targeted molecular imaging of fibrin, a selection of fibrin-specific aptamer would be recommended. A close analysis of the binding interaction of fibrin-targeting peptides with the fibrin network could assist in the design of the fibrin aptamer SELEX parameters. This aptamer can then be modified or conjugated in the same manner as outlined in this research, and its efficacy can be compared to that of peptidetargeted contrast formulations. With gadolinium-conjugated materials, it would be beneficial to conduct transmetalation studies, to determine if NOTA chelators are stable enough to be translated to in vivo studies. In addition, gadolinium nanoparticles may be an effective alternative to the chelator-based formulation, as these nanoparticles consist of a large number of the paramagnetic ion. For the dual-contrast formulation, it may be beneficial to explore methods that would allow for deposition of a thicker gold coating, which can be subsequently tested in $\mathrm{CT}$ imaging. For this, it may be necessary to test a range of coating thicknesses. In addition, nanoagents should be characterized using powder $\mathrm{XRD}$ for phase identification of these crystalline materials and inductively coupled plasma mass spectrometry to verify mass size distribution. In all formulations, it would be recommended to explore nucleotide modifications that will increase stability for future in vivo studies. As an example, locked nucleotides may be implemented and tested with nucleases to confirm stability. Once a fibrin-binding aptamer is selected, in an effort to lower the production cost, minimers should be further explored and their binding should 
be compared to that of the full strand with both types of target, solution-based fibrin and blood clots. 


\section{References}

(1) Crick, F. Nature 1970, 227, 561-563.

(2) Gilbert, W. Nature 1986, 319, 618.

(3) Zaug, A. J.; Cech, T. R. Science (80). 1986, 231 (4737), 470-475.

(4) Guerrier-Takada, C.; Gardiner, K.; Marsh, T.; Pace, N.; Altman, S. Cell 1983, 35 (3 Pt 2), 849-857.

(5) Ellington, A.; Szostak, J. Nature 1990, 346, 818-822.

(6) Tuerk, C.; Gold, L. Science 1990, 249 (4968), 505-510.

(7) Robertson, D. L.; Joyce, G. F. Nature 1990, 344 (6265), 467-468.

(8) Gold, L.; Janjic, N.; Jarvis, T.; Schneider, D.; Walker, J. J.; Wilcox, S. K.; Zichi, D. Cold Spring Harb. Perspect Biol 2012, 4 (3), 1-9.

(9) Garst, A. D.; Edwards, A. L.; Batey, R. T. Cold Spring Harb Perspect Biol 2011, 3 (6), 1-13.

(10) Colas, P.; Cohen, B.; Jessen, T.; Grishina, I.; Mccoyt, J.; Brent, R. Nature 1996, $380,548-550$.

(11) Stoltenburg, R.; Reinemann, C.; Strehlitz, B. Biomol. Eng. 2007, 24 (4), 381-403.

(12) Ruigrok, V. J. B.; Levisson, M.; Eppink, M. H. M.; Smidt, H.; Van Der Oost, J. Biochem. J. 2011, 436 (1), 1-13.

(13) Keefe, A. D.; Pai, S.; Ellington, A. Nat. Rev. Drug Discov. 2010, 9 (7), 537-550.

(14) Bernard, E. D.; Beking, M. A.; Rajamanickam, K.; Tsai, E. C.; DeRosa, M. C. J. Biol. Inorg. Chem. 2012, 17 (8), 1159-1175.

(15) Jenison, R. D.; Gill, S. C.; Pardi, A.; Polisky, B. Science (80). 1994, 263 (5152), $1425-1429$. 
(16) Cruz-Toledo, J.; McKeague, M.; Zhang, X.; Giamberardino, A.; McConnell, E.; Francis, T.; DeRosa, M. C.; Dumontier, M. Database 2012, 2012, 1-8.

(17) Tolle, F.; Mayer, G. Chem. Sci. 2013, 4 (1), 60-67.

(18) Mayer, G. Angew. Chemie - Int. Ed. 2009, 48 (15), 2672-2689.

(19) Zhang, Y.; Lai, B. S.; Juhas, M. Molecules 2019, 24 (5), 1-22.

(20) Blind, M.; Blank, M. Mol. Ther. - Nucleic Acids 2015, 4 (1), e223.

(21) Darmostuk, M.; Rimpelova, S.; Gbelcova, H.; Ruml, T. Biotechnol. Adv. 2015, 33 (6 pt 2), 1141-1161.

(22) McKeague, M.; Derosa, M. C. J. Nucleic Acids 2012, 2012, 748913.

(23) Bruno, J. G. Pharmaceuticals 2013, 6 (3), 340-357.

(24) Ni, S.; Yao, H.; Wang, L.; Lu, J.; Jiang, F.; Lu, A.; Zhang, G. Int. J. Mol. Sci. 2017, $18(8)$.

(25) Moreno, A.; Pitoc, G. A.; Ganson, N. J.; Layzer, J. M.; Hershfield, M. S.; Tarantal, A. F.; Sullenger, B. A. Cell Chem. Biol. 2019, 26 (5), 634-644.e3.

(26) Kattula S; JR, B.; Wolberg AS. Arterioscler. Thromb. Vasc. Biol. 2017, 37 (3), e13-e21.

(27) Wolberg, A. S. Blood Rev. 2007, 21 (3), 131-142.

(28) Li, M.; Lin, N.; Huang, Z.; Du, L.; Altier, C.; Fang, H. J. Am. Chem. Soc. 2008, $130(38), 12636-12638$.

(29) Adamczyk, B.; Struwe, W. B.; Ercan, A.; Nigrovic, P. A.; Rudd, P. M. J. Proteome Res. 2013, 12 (1), 444-454.

(30) Guedes, A. F.; Carvalho, F. A.; Domingues, M. M.; Macrae, F. L.; McPherson, H. R.; Sabban, A.; Martins, I. C.; Duval, C.; Santos, N. C.; Ariens, R. A. 
Nanomedicine 2018, 13 (19), 2491-2505.

(31) Stassen, J.; Arnout, J.; Deckmyn, H. Curr. Med. Chem. 2012, 11 (17), 2245-2260.

(32) Krishnaswamy S. J Thromb Haemost 2013, 11 (1), 265-276.

(33) Gale, A. J. Toxicol. Pathol. 2011, 39 (1), 273-280.

(34) Lipets, E. N.; Ataullakhanov, F. I. Thromb. J. 2015, 13 (1), 1-15.

(35) Schafer, A. I. Ann. Intern. Med. 1985, 102, 814-828.

(36) Nakashima, M. O.; Rogers, H. J. Blood Res. 2014, 49 (2), 85-94.

(37) Vilar, R.; Fish, R. J.; Casini, A.; Neerman-Arbez, M. Haematologica 2020, 105 (2), 284-296.

(38) Donkor, E. S. Stroke Res. Treat. 2018, 2018, 1-10.

(39) Gerard, C.; Busl, K. M. Curr. Treat. Options Neurol. 2014, 16 (1).

(40) Wilkinson, D. A.; Pandey, A. S.; Thompson, B. G.; Keep, R. F.; Hua, Y.; Xi, G. Neuropharmacology 2018, 134, 240-248.

(41) Caceres, J. A.; Goldstein, J. N. Emerg. Med. Clin. North Am. 2012, 30 (3), 771794.

(42) Brown, R. J.; Broderick, J. P. Lancet Neurol. 2014, 13 (4), 393-404.

(43) Etminan, N.; Rinkel, G. J. Nat. Rev. Neurol. 2016, 12 (12), 699-713.

(44) van Gijn, J.; Kerr, R. S.; Rinkel, G. J. E. Lancet 2007, 369, 306-318.

(45) Keedy, A. McGill J. Med. 2006, 9 (2), 141-146.

(46) Gasparotti, R.; Liserre, R. Eur. Radiol. 2005, 15 (3), 441-447.

(47) Danière, F.; Gascou, G.; de Champfleur, N. M.; Machi, P.; Leboucq, N.; Riquelme, C.; Ruiz, C.; Bonafé, A.; Costalat, V. Diagn. Interv. Imaging 2015, 96 (7-8), 677-686. 
(48) Overoye-Chan, K.; Koerner, S.; Looby, R. J.; Kolodziej, A. F.; Zech, S. G.; Deng, Q.; Chasse, J. M.; McMurry, T. J.; Caravan, P. J. Am. Chem. Soc. 2008, 130 (18), $6025-6039$.

(49) Catanese, L.; Tarsia, J.; Fisher, M. Circ. Res. 2017, 120, 541-558.

(50) Deb, P.; Sharma, S.; Hassan, K. M. Pathophysiology 2010, 17 (3), 197-218.

(51) Ng, F. C.; Campbell, B. C. V. Curr. Neurol. Neurosci. Rep. 2019, 19 (8).

(52) Kamalian, S.; Lev, M. H. Radiol. Clin. North Am. 2019, 57 (4), 717-732.

(53) Rogosnitzky, M.; Branch, S. BioMetals 2016, 29 (3), 365-376.

(54) Chen, Z.; Wang, Y.; Lin, Y.; Zhang, J.; Yang, F.; Zhou, Q.; Liao, Y. Biomed Res. Int. 2014, 2014, 1-12.

(55) Wallyn, J.; Anton, N.; Akram, S.; Vandamme, T. F. Pharm. Res. 2019, 36 (78), 134.

(56) Bae, K. T. Radiology 2010, 256 (1), 32-61.

(57) Ramalho, J.; Semelka, R. C.; Ramalho, M.; Nunes, R. H.; AlObaidy, M.; Castillo, M. Am. J. Neuroradiol. 2016, 37 (7), 1192-1198.

(58) Andreucci, M.; Faga, T.; Serra, R.; De Sarro, G.; Michael, A. Drug. Healthc. Patient Saf. 2017, 9, 25-37.

(59) Warram, J.; de Boer, E.; Sorace, A.; Chung, T.; Kim, H.; Pleijhuis, R.; van Dam, G.; Rosenthal, E. Cancer Metastasis Rev 2015, 33 (0), 809-822.

(60) Vithanarachchi, S. M.; Allen, M. J. Curr Mol Imaging 2013, 1 (1), 12-25.

(61) Tolmachev, V.; Orlova, A. Cancers (Basel). 2020, 12 (3), 1-21.

(62) Jokerst, J. V.; Miao, Z.; Zavaleta, C.; Cheng, Z.; Gambhir, S. S. Small 2011, 7 (5), $625-633$. 
(63) Gao, J.; Chen, K.; Miao, Z.; Ren, G.; Chen, X.; Gambhir, S. S.; Chenga, Z. Biomaterials 2011, 32 (8), 2141-2148.

(64) Ståhl, S.; Gräslund, T.; Eriksson Karlström, A.; Frejd, F. Y.; Nygren, P. Å.; Löfblom, J. Trends Biotechnol. 2017, 35 (8), 691-712.

(65) Rosen, J. E.; Yoffe, S.; Meerasa, A.; Verma, M.; Gu, F. X. J. Nanomed. Nanotechnol. 2011, 2 (5), 1-12.

(66) Nakamura, Y.; Mochida, A.; Choyke, P. L.; Kobayashi, H. Bioconjug. Chem. 2016, 27 (10), 2225-2238.

(67) Wang, L.; Huang, J.; Chen, H.; Wu, H.; Xu, Y.; Li, Y.; Yi, H.; Wang, Y. A.; Yang, L.; Mao, H. ACS Nano 2017, 11 (5), 4582-4592.

(68) Li, L.; Jiang, W.; Luo, K.; Song, H.; Lan, F.; Wu, Y.; Gu, Z. Theranostics 2013, 3 (8), 595-615.

(69) Bean, C.; Livingston, J. J. Appl. Phys. 1959, 30 (4), 120-129.

(70) Laurent, S.; Elst, L. Vander; Muller, R. N. Chem. Contrast Agents Med. Magn. Reson. Imaging Second Ed. 2013, 427-447.

(71) You, J.; Shi, M.; Dong, L.; Xu, K. Int. J. Nanomedicine 2018, 13, 4607-4625.

(72) Dai, Y.; Wu, C.; Wang, S.; Li, Q.; Zhang, M. Nanomedicine Nanotechnology, Biol. Med. 2018, 14, 547-555.

(73) Pellico, J.; Ellis, C. M.; Davis, J. J. Contrast Media Mol. Imaging 2019, 2019, 113.

(74) Xia, Y.; Xu, C.; Zhang, X.; Ning, P.; Wang, Z.; Tian, J.; Chen, X. Nanoscale 2019, $11,5822-5838$.

(75) Xi, D.; Dong, S.; Meng, X.; Lu, Q.; Meng, L.; Ye, J. RSC Adv. 2012, 2 (33), 
$12515-12524$.

(76) Mahan, M. M.; Doiron, A. L. J. Nanomater. 2018, 2018, 1-15.

(77) Wang, R.; Ms, M.; Deng, J.; He, D.; Yang, E.; Ms, M.; Yang, W.; Ms, M.; Shi, D.; Jiang, Y.; Qiu, Z.; Webster, T. J.; Shen, Y. Nanomedicine Nanotechnology, Biol. Med. 2019, 16, 195-205.

(78) Clough, T. J.; Baxan, N.; Coakley, E. J.; Rivas, C.; Zhao, L.; Leclerc, I.; MartinezSanchez, A.; Rutter, G. A.; Long, N. J. RSC Dalt. Trans. 2020, 49 (15), 4732 4740.

(79) Schuerle, S.; Furubayashi, M.; Soleimany, A. P.; Gwisai, T.; Huang, W.; Voigt, C.; Bhatia, S. N. ACS Synth. Biol. 2020, 9, 392-401.

(80) Chen, Y.; Fu, Y.; Li, X.; Chen, H.; Wang, Z.; Zhang, H. RSC Adv. 2019, 9 (30), 17093-17100.

(81) Journal, A. I.; Xu, W.; Zhang, S.; Zhou, Q.; Chen, W. Artif. Cells, Nanomedicine, Biotechnol. 2019, 47 (1), 2440-2448.

(82) Ahmad, M. Y.; Ahmad, M. W.; Cha, H.; Oh, I.-T.; Tegafaw, T.; Miao, X.; Ho, S. L.; Marasini, S.; Ghazanfari, A.; Yue, H.; Ryeom, H.-K.; Lee, J.; Chae, K. S.; Lee, G. H. Eur. J. Inorg. Chem. 2018, 2018, 3070-3079.

(83) Tsvirkun, D.; Ben-Nun, Y.; Merquiol, E.; Zlotver, I.; Meir, K.; Weiss-Sadan, T.; Matok, I.; Popovtzer, R.; Blum, G. J. Am. Chem. Soc. 2018, 140, 12010-12020.

(84) Wu, M.; Zhang, Y.; Zhang, Y.; Wu, M.; Wu, M.; Wu, H.; Cao, L.; Li, L.; Li, X.; Zhang, X. RSC Adv. 2018, 8, 1706-1716.

(85) Li, X.; Wu, M.; Wang, J.; Dou, Y. Nanomedicine Nanotechnology, Biol. Med. 2019, $15(1), 252-263$. 
(86) Feng, Y.; Chen, H.; Shao, B.; Zhao, S.; Wang, Z.; You, H. ACS Appl. Bio Mater. 2018, 10, 25511-25518.

(87) Wang, T.; Jia, G.; Cheng, C.; Wang, Q.; Li, X.; Liu, Y.; He, C.; Chen, L.; Suna, G.; Zuo, C. R. Soc. Chem. 2018, 42, 11565-11572.

(88) Zu, G.; Cao, Y.; Dong, J.; Zhou, Q.; Rijn, P. Van; Liu, M.; Pei, R. ACS Appl. Bio Mater. 2018, 2, 406-416.

(89) Zhang, L.-X.; Li, K.-F.; Wang, H.; Gu, M.-J.; Liu, L.-S.; Zheng, Z.-Z.; Han, N.-Y.; Yang, Z.-J.; Fan, T.-Y. AAPS PharmChiTech 2017, 18 (5), 1564-1571.

(90) Huang, X.; Zhong, J.; Ren, J.; Wen, D.; Zhao, W.; Huan, Y. Oncol. Lett. 2019, 18, $265-274$.

(91) Yan, H.; Gao, X.; Zhang, Y.; Chang, W.; Li, J.; Li, X.; Du, Q.; Li, C. ACS Appl. Mater. Interfaces 2018, 10, 17047-17057.

(92) Zhong, L.; Zou, H.; Huang, Y.; Gong, W.; He, J.; Tan, J.; Lai, Z.; Li, Y. J. Biomed. Nanotechnol. 2019, 15 (2), 352-362.

(93) Fang, Y.; Lin, S.; Yang, F.; Situ, J.; Lin, S.; Luo, Y. Biomed Res. Int. 2020, 2020, $1-12$.

(94) Cao, Y.; Wu, T.; Dai, W.; Dong, H.; Zhang, X. Chem. Mater. 2019, 31, 91059114.

(95) Alibolandi, M.; Hoseini, F.; Mohammadi, M.; Ramezani, P.; Einafshar, E.; Taghdisi, S. M.; Ramezani, M.; Abnous, K. Int. J. Pharm. 2018, 549 (1-2), 67-75.

(96) Mohammadzadeh, P.; Cohan, R. A.; Ghoreishi, S. M.; Bitarafan-Rajabi, A.; Ardestani, M. S. Sci. Rep. 2017, 7 (1), 1-16.

(97) Harris, D. C. Quantitative Chemical Analysis, 8th ed.; 2010. 
(98) Blackburn, G. M.; Gait, M. J.; Loakes, D.; Williams, D. Nucleic Acids in Chemistry and Biology; Royal Society of Chemistry, 2006.

(99) Kypr, J.; Kejnovská, I.; Renčiuk, D.; Vorlíčková, M. Nucleic Acids Res. 2009, 37 (6), 1713-1725.

(100) Chang, Y.; Chen, C. K.; Hou, M. Int. J. Mol. Sci. 2012, 13, 3394-3413.

(101) Kelly, S.; Price, N. Curr. Protein Pept. Sci. 2005, 1 (4), 349-384.

(102) Jing, M.; Bowser, M. T. Anal. Chim. Acta 2011, 686 (1-2), 9-18.

(103) Johnson, W. C. Circ. Dichroism Conform. Anal. Biomol. 1996, 433-468.

(104) Freyer, M. W.; Lewis, E. A. Methods Cell Biol. 2008, 84 (07), 79-113.

(105) Freire, E.; Mayorga, O. L.; Straume, M. Anal. Chem. 1990, 62 (18), 950A-959A.

(106) Duhr, S.; Braun, D. Proc. Natl. Acad. Sci. U. S. A. 2006, 103 (52), 19678-19682.

(107) Jerabek-Willemsen, M.; André, T.; Wanner, R.; Roth, H. M.; Duhr, S.; Baaske, P.; Breitsprecher, D. J. Mol. Struct. 2014, 1077, 101-113.

(108) Fontaine, S. D.; Reid, R.; Robinson, L.; Ashley, G. W.; Santi, D. V. Bioconjug. Chem. 2015, $26(1), 145-152$.

(109) Gijs, M.; Dammicco, S.; Warnier, C.; Aerts, A.; Impens, N. R. E. N.; D’Huyvetter, M.; Léonard, M.; Baatout, S.; Luxen, A. J. Label. Compd. Radiopharm. 2016, 59 (2), 63-71.

(110) Liu, G.; Dou, S.; Liu, Y.; Liang, M.; Chen, L.; Cheng, D.; Greiner, D.; Rusckowski, M.; Hnatowich, D. Nucl Med Biol 2011, 38 (2), 1-11.

(111) Getz, E. B.; Xiao, M.; Chakrabarty, T.; Cooke, R.; Selvin, P. R. Anal. Biochem. 1999, 273 (1), 73-80.

(112) Häkkinen, H. Nat. Chem. 2012, 4 (6), 443-455. 
(113) Li, F.; Zhang, H.; Dever, B.; Li, X. F.; Le, X. C. Bioconjug. Chem. 2013, 24 (11), $1790-1797$.

(114) Barge, A.; Cravotto, G.; Gianolio, E.; Fedeli, F. Contrast Media Mol. Imaging 2006, $1,184-188$.

(115) Gunning, W. T.; Calomeni, E. P. J. Histotechnol. 2000, 23 (3), 237-246.

(116) Fultz, B.; Howe, J. M. Transmission Electron Microscopy and Diffractometry of Materials; 3rd, Ed.; Springer-Verlag Berlin Heidelberg, 2008.

(117) Eskandari, M. J.; Gostariani, R. Transmission Electron Microscopy of Nanomaterials. In Electron Crystallography; 2020.

(118) Hodoroaba, V. D. Energy-Dispersive X-Ray Spectroscopy (EDS); Elsevier Inc., 2020.

(119) Scimeca, M.; Bischetti, S.; Lamsira, H. K.; Bonfiglio, R.; Bonanno, E. Eur. J. Histochem. 2018, 62 (1), 89-99.

(120) Wang, M. C.; Pins, G. D.; Silver, F. H. Mater. Sci. Eng. C 1995, 3 (2), 131-135.

(121) Spotnitz, W. D. ISRN Surg. 2014, 2014, 1-28.

(122) Grover, V. P. B.; Tognarelli, J. M.; Crossey, M. M. E.; Cox, I. J.; Taylor-robinson, S. D.; Mcphail, M. J. W. J. Clin. Exp. Hepatol. 2015, 5 (3), 246-255.

(123) Weishaupt, D.; Kochli, V. D.; Marincek, B. How Does MRI Work? An Introduction to the Physics and Function of Magnetic Resonance Imaging; Springer-Verlag Berlin Heidelberg, 2006.

(124) Plewes, D. B.; Kucharczyk, W. J. Magn. Reson. Imaging 2012, 35 (5), 1038-1054.

(125) Liney, G. MRI in Clinical Practice; Springer-Verlag London Limited, 2006.

(126) McMahon, K. L.; Cowin, G.; Galloway, G. J. Orthop. Sports Phys. Ther. 2011, 41 
(11), 806-819.

(127) Shah, N. J.; Mo, K. Neuromethods 2012, 71, 75-98.

(128) Smith, R. C.; Lange, R. C. Understanding Magnetic Resonance Imaging; America, C. P. L. U. S. of, Ed.; 1998.

(129) Webb, A. Introduction to Biomedical Imaging, IEEE Press.; John Wiley \& Sons, Inc.

(130) Freeman, R. Magnetic Resonance in Chemistry and Medicine; Oxford University Press, 2003.

(131) Kuperman, V. Magnetic Resonance Imaging: Physical Principles and Applicatoins; Academic Press: A Harcourt Science and Technology Company, 2000.

(132) Jeol. JNM-ECA II/JNM-ECX II/JNM-ECS Series Applications User’s Manual.

(133) Caravan, P.; Ellison, J. J.; McMurry, T. J.; Lauffer, R. B. Chem. Rev. 1999, 99 (9), $2293-2352$.

(134) De Leõn-Rodríguez, L. M.; Martins, A. F.; Pinho, M. C.; Rofsky, N. M.; Sherry, A. D. Basic MR Relaxation Mechanisms and Contrast Agent Design; 2015; Vol. 42.

(135) Price, E. W.; Orvig, C. Chem. Soc. Rev. 2014, 43 (1), 260-290.

(136) Pyykko, P. Nat. Chem. 2015, 7 (August), 2015.

(137) Toth, E.; Helm, L.; Merbach, A. Relaxivity of Gadolinium (III) Complexes: Theory and Mechanism. In The Chemistry of Contrast Agents in Medical Magnetic Resonance Imaging; John Wiley \& Sons, Ltd, 2013; pp 26-81.

(138) Jacques, V.; Dumas, S.; Sun, W.-C.; Troughton, J. S.; Greenfield, M. T.; Caravan, 
P.; Manuscript, A. Invest. Radiol. 2010, 45 (10), 613-624.

(139) Wahsner, J.; Gale, E. M.; Rodríguez-Rodríguez, A.; Caravan, P. Chem. Rev. 2019, $119(2), 957-1057$.

(140) Vallabani, N. V. S.; Singh, S.; Karakoti, A. S. Curr. Drug Metab. 2018, 20 (6), $457-472$.

(141) Goldman, L. W. J. Nucl. Med. Technol. 2009, 35 (3), 115-129.

(142) Cogbill, T. H.; Ziegelbein, K. J. Surg. Clin. North Am. 2011, 91 (1), 1-14.

(143) Lusic, H.; Grinstaff, M. W. Chem Riv 2013, 113 (3), 1-64.

(144) Yeh, B. M.; FitzGerald, P. F.; Edic, P. M.; Lambert, J. W.; Colborn, R. E.; Marino, M. E.; Evans, P. M.; Roberts, J. C.; Wang, Z. J.; Wong, M. J.; Bonitatibus, P. J., J. Adv. Drug Deliv. Rev. 2017, 113, 201-222.

(145) Ginat, D. T.; Gupta, R. Annu Rev Biomed Eng 2014, 16, 431-453.

(146) Singh, J.; Daftary, A. J. Nucl. Med. Technol. 2008, 36 (2), 69-74.

(147) Bae, K. T. Radiology 2010, 256 (1), 32-61.

(148) Schneider, C. A.; Rasband, W. S.; Eliceiri, K. W. Nat. Methods 2012, 9 (7), 671675.

(149) Collins, T. J. Biotechniques 2007, 43 (1S), S25-S30.

(150) Bompiani, K. M.; Lohrmann, J. L.; Pitoc, G. A.; Frederiksen, J. W.; Mackensen, G. B.; Sullenger, B. A. Chem. Biol. 2014, 21 (8), 935-944.

(151) Woodruff, R. S.; Sullenger, B. A. Arterioscler. Thromb. Vasc. Biol. 2015, 35 (10), 2083-2091.

(152) Srinivasan, V. M.; Chen, S. R.; Camstra, K. M.; Chintalapani, G.; Kan, P. Neurosurg. Focus 2017, 42 (4), 1-6. 
(153) Pera, J.; Undas, A.; Topor-Madry, R.; Jagiella, J.; Klimkowicz-Mrowiec, A.; Slowik, A. Stroke 2012, 43 (5), 1412-1414.

(154) Kim, J.; Park, J. E.; Nahrendorf, M.; Kim, D. E. J. Stroke 2016, 18 (3), 286-296.

(155) Edelman, R. R.; Warach, S. N. Engl. J. Med. 1993, 328 (10), 708-716.

(156) Choi, J. W.; Moon, W. J. Korean J. Radiol. 2019, 20 (1), 134-147.

(157) Klemm, P. J.; Floyd 3rd, W. C.; Smiles, D. E.; Fréchet, J. M. J.; Raymond, K. N.; Manuscript, A. Contrast Media Mol. Imaging 2012, 7 (1), 95-99.

(158) Xiao, Y. D.; Paudel, R.; Liu, J.; Ma, C.; Zhang, Z. S.; Zhou, S. K. Int. J. Mol. Med. 2016, $38(5), 1319-1326$.

(159) Lee, M. J.; Kim, M. J.; Yoon, C. S.; Song, S. Y.; Park, K.; Kim, W. S. Korean J. Radiol. 2011, 12 (3), 358-364.

(160) Hadjiathanasiou, A.; Schuss, P.; Brandecker, S.; Welchowski, T.; Schmid, M.; Vatter, H.; Güresir, E. BMC Neurol. 2020, 20 (1), 1-12.

(161) Yu, S.; Watson, A. D.; Bismuth, B. Chem Riv 1999, 99 (9), 2353-2377.

(162) Ponce, A. T.; Hong, K. L. Biomedicines 2019, 7 (3).

(163) Soule, E. E.; Bompiani, K. M.; Woodruff, R. S.; Sullenger, B. A. Nucleic Acid Ther. 2016, 26 (1), 1-9.

(164) Andia, M. E.; Saha, P.; Jenkins, J.; Modarai, B.; Wiethoff, A. J.; Phinikaridou, A.; Grover, S. P.; Patel, A. S.; Schaeffter, T.; Smith, A.; Botnar, R. M. Arterioscler. Thromb. Vasc. Biol. 2014, 34 (6), 1193-1198.

(165) Sirol, M.; Fuster, V.; Badimon, J. J.; Fallon, J. T.; Moreno, P. R.; Toussaint, J. F.; Fayad, Z. A. Circulation 2005, 112 (11), 1594-1600.

(166) Vymazal, J.; Spuentrup, E.; Cardenas-Molina, G.; Wiethoff, A. J.; Hartmann, M. 
G.; Caravan, P.; Parsons, E. C. J. Invest. Radiol. 2009, 44 (11), 697-704.

(167) Ciesienski, K. L.; Yang, Y.; Ay, I.; Chonde, D. B.; Loving, G. S.; Rietz, T. A.; Catana, C.; Caravan, P. Mol Pharm 2013, 10 (3), 1100-1110.

(168) Hamilton, C. J.; Germann, M. W. Colon. Acad. Alliance Undergrad. Res. J. 2011, $2(11), 1-14$.

(169) McKeague, M.; Bradley, C. R.; de Girolamo, A.; Visconti, A.; David Miller, J.; DeRosa, M. C. Int. J. Mol. Sci. 2010, 11 (12), 4864-4881.

(170) Hisada, Y.; Yasunaga, M.; Hanaoka, S.; Saijou, S.; Sugino, T.; Tsuji, A.; Saga, T.; Tsumoto, K.; Manabe, S.; Kuroda, J. I.; Kuratsu, J. I.; Matsumura, Y. Sci. Rep. 2013, 3,1-7.

(171) Terasawa, F.; Kani, S.; Hongo, M.; Okumura, N. Thromb. Res. 2006, 118 (5), $651-661$.

(172) Birenbaum, D.; Bancroft, L. W.; Felsberg, G. J. West. J. Emerg. Med. 2011, 12 (1), $67-76$.

(173) Xu, W.; Lu, Y. Chem. Commun. 2011, 47 (17), 4998-5000.

(174) Yigit, M. V.; Mazumdar, D.; Lu, Y. Bioconjug. Chem. 2008, 19, 412-417.

(175) Zhang, K.; Cao, Y.; Pei, R.; Zhang, Y.; Zhang, T.; Liu, M.; Kuang, Y.; Zu, G.; Zhang, K.; Cao, Y.; Pei, R. J. Nanosci. Nanotechnol. 2018, 18 (6), 3759-3774.

(176) Li, J.; Li, Q.; He, M.; Ding, F.; Cai, L.; Zhao, M.; Dong, L.; Wang, Q.; Xu, K. RSC Adv. 2019, 9 (60), 34837-34846.

(177) Gu, M. J.; Li, K. F.; Zhang, L. X.; Wang, H.; Liu, L. S.; Zheng, Z. Z.; Han, N. Y.; Yang, Z. J.; Fan, T. Y. Int. J. Nanomedicine 2015, 10, 5187-5204.

(178) Geraldes, C. F. G. C.; Sherry, A. D.; Brown, R. D. Magn. Reson. Med. 1986, 3, 
$242-250$.

(179) Kumar, A.; Zhang, S.; Hao, G.; Hassan, G.; Ramezani, S.; Sagiyama, K. Bioconjug. Chem. 2015, 26, 549-558.

(180) Knop, R.; Frank, J.; Dwyer, A.; Girton, M.; Naegele, M.; Schrader, M.; Cobb, J.; Gansow, O.; Maegerstadt, M.; Brechbiel, M.; Baltzer, L.; Doppman, J. J. Comput. Assist. Tomogr. 1987, 11 (1), 35-42.

(181) Caravan, P. Chem. Soc. Rev. 2006, 35 (6), 512-523.

(182) Rohrer, M.; Bauer, H.; Mintorovitch, J. Invest. Radiol. 2005, 40 (11), 715-724.

(183) Rashid, H. U.; Martines, M. A. U.; Jorge, J.; de Moraes, P. M.; Umar, M. N.; Khan, K.; Rehman, H. U. Bioorganic Med. Chem. 2016, 24 (22), 5663-5684.

(184) Geraldes, C. F. G. C.; Brown, R. D.; Brucher, E.; Koenig, S. H.; Sherry, A. D.; Spiller, M. Magn. Reson. Med. 1992, 27 (2), 284-295.

(185) Rainard, J. M.; Pandarakalam, G. C.; Mcelroy, S. P. SLAS Discov. 2018, 23 (3), $225-241$.

(186) Scheuermann, T. H.; Padrick, S. B.; Gardner, K. H.; Brautigam, C. A. Anal. Biochem. 2016, 496, 79-93.

(187) Kretschy, N.; Sack, M.; Somoza, M. M. Bioconjug. Chem. 2016, 27 (3), 840-848.

(188) Collen, D.; Kudryk, B.; Hessel, B.; Blomback, B. J. Biol. Chem. 1975, 250 (15), $5808-5817$.

(189) Bhattacharjee, A.; Bansal, M. 2005, 57 (March), 161-172.

(190) Shoulders, M. D.; Raines, R. T. 2009, 929-958.

(191) Christoffersson, J. O.; Olsson, L. E.; Sjöberg, S. Acta radiol. 1991, 32 (5), 426431. 
(192) Weinmann, H.-J.; Brasch, R. C.; Press, W.-R.; Wesbey, G. E. AJR 1984, 142 (3), $619-624$.

(193) Heese, F.; Robson, P.; Hall, L. D. IEEE Sens. J. 2005, 5 (2), 277-280.

(194) Jeon, S. B.; Kang, D. W. J. Neurol. Neurosurg. Psychiatry 2007, 78 (8), 871.

(195) Asselta, R.; Duga, S.; Tenchini, M. L. J. Thromb. Haemost. 2006, 4 (10), 21152129.

(196) Caravan, P. Acc. Chem. Res. 2009, 42 (7), 851-862.

(197) Wufsus, A. R.; Macera, N. E.; Neeves, K. B. Biophys. J. 2013, 104 (8), $1812-$ 1823.

(198) Merbach, A.; Helm, L.; Toth, E. The Chemistry of Contrast Agents in Medical Magnetic Resonance Imaging, 2nd ed.; Wiley, Ed.; 2013.

(199) Cheng, C.; Chen, Y. H.; Lennox, K. A.; Behlke, M. A.; Davidson, B. L. Mol. Ther. - Nucleic Acids 2013, 2 (e67), 1-9.

(200) McConnell, E. M.; Ventura, K.; Dwyer, Z.; Hunt, V.; Koudrina, A.; Holahan, M. R.; Derosa, M. C. ACS Chem. Neurosci. 2019, 10 (1), 371-383.

(201) Macdonald, J.; Henri, J.; Goodman, L.; Xiang, D.; Duan, W.; Shigdar, S. ACS Chem. Neurosci. 2017, 8 (4), 777-784.

(202) Alias, S.; Lang, I. M. Pulm. Circ. 2013, 3 (4), 728-738.

(203) Zuker, M. Nucleic Acids Res. 2003, 31 (13), 3406-3415.

(204) Wang, X.; Liu, Z.; Pang, Y. R. Soc. Chem. Adv. 2017, 7, 29966-29984.

(205) Hoffman, M.; Monroe III, D. M. Thromb Haemost 2001, 85, 958-965.

(206) Blasi, F.; Oliveira, B. L.; Rietz, T. A.; Rotile, N. J.; Naha, P. C.; Cormode, D. P.; Izquierdo-garcia, D.; Catana, C.; Caravan, P. Arterioscler. Thromb. Vasc. Biol. 
2015, 35 (10), 2114-2121.

(207) Fleck, D.; Albadawi, H.; Shamoun, F.; Knuttinen, G.; Naidu, S.; Oklu, R. Cardiovasc. Diagn. Ther. 2017, 7 (Suppl 3), S228-S237.

(208) Mitchell, B. D.; Chinnadurai, P.; Chintalapani, G.; Morsi, H. A.; Shaltoni, H.; Mawad, M. E. Am. J. Neuroradiol. 2015, 36 (7), 1317-1320.

(209) Cormode, D. P.; Naha, P. C.; Fayad, Z. A. Contrast Media Mol Imaging 2014, 9 (1), 37-52.

(210) Koudrina, A.; DeRosa, M. C. ACS Omega 2020, Manuscript ID: ao-202002650r.R2.

(211) Dougherty, C. A.; Cai, W.; Hong, H. Curr Top Med Chem 2015, 15 (12), 1138 1152.

(212) Hernandez, C.; Birktoft, J. J.; Ohayon, Y. P.; Chandrasekaran, A. R.; Abdallah, H.; Sha, R.; Stojanoff, V.; Mao, C.; Seeman, N. C. Cell Chem. Biol. 2017, 24 (11), 1401-1406.e2.

(213) Ferrer, E.; Wiersma, M.; Kazimierczak, B.; Müller, C. W.; Eritja, R. Bioconjug. Chem. 1997, 8 (5), 757-761.

(214) Toyohara, J.; Hayashi, A.; Sato, M.; Tanaka, H.; Haraguchi, K.; Yoshimura, Y.; Yonekura, Y.; Fujibayashi, Y. J. Nucl. Med. 2002, 43 (9), 1218-1226.

(215) Koudrina, A.; McConnell, E. M.; Zurakowski, J. A.; Cron, G. O.; Chen, S.; Tsai, E. C.; DeRosa, M. C. ACS Appl. Mater. Interfaces 2020, Manuscript ID: am-2020166668.

(216) Liu, B.; Liu, J. Anal. Methods 2017, 9 (18), 2633-2643.

(217) Gounis, M. J.; Nogueira, R. G.; Mehra, M.; Chueh, J.; Wakhloo, A. K. J. 
Neurointerv. Surg. 2013, 5 (suppl 1), i85 LP-i89.

(218) Thavarajah, R.; Mudimbaimannar, V. K.; Elizabeth, J.; Rao, U. K.; Ranganathan, K. Chemical and Physical Basics of Routine Formaldehyde Fixation. Journal of Oral and Maxillofacial Pathology. 2012, pp 400-405.

(219) Odeh, F.; Nsairat, H.; Alshaer, W.; Ismail, M. A.; Esawi, E.; Qaqish, B.; Bawab, A. A.; Ismail, S. I. Molecules 2019, 25 (3), 1-51.

(220) Kim, J. Y.; Ryu, J. H.; Schellingerhout, D.; Sun, I. C.; Lee, S. K.; Jeon, S.; Kim, J.; Kwon, I. C.; Nahrendorf, M.; Ahn, C. H.; Kim, K.; Kim, D. E. Theranostics 2015, $5(10), 1098-1114$.

(221) Chiu, T. C.; Huang, C. C. Sensors 2009, 9 (12), 10356-10388.

(222) Rippe, K. BIF Futur. 1997, 12, 20-26.

(223) McKeague, M.; De Girolamo, A.; Valenzano, S.; Pascale, M.; Ruscito, A.; Velu, R.; Frost, N. R.; Hill, K.; Smith, M.; McConnell, E. M.; DeRosa, M. C. Anal. Chem. 2015, 87 (17), 8608-8612.

(224) Sakamoto, T.; Ennifar, E.; Nakamura, Y. Biochimie 2018, 145, 91-97.

(225) Raman, S. P.; Mahesh, M.; Blasko, R. V; Fishman, E. K. JACR 2013, 10 (11), $840-846$.

(226) Zhang, H.; Shang, Y.; Li, Y. H.; Sun, S. K.; Yin, X. B. ACS Appl. Mater. Interfaces 2019, 11 (2), 1886-1895.

(227) Beckett, K. R.; Moriarity, A. K.; Langer, J. M. Radiographics 2015, 35 (6), 17381750.

(228) Sanghavi, P. S.; Jankharia, B. G. Indian J. Radiol. Imaging 2019, 29 (3), 289-298. (229) Aleman, M. M.; Walton, B. L.; Byrnes, J. R.; Wolberg, A. S. Thromb. Res. 2014, 
$133(1), 1-7$.

(230) Nute, J. L.; Roux, L. Le; Chandler, A. G.; Baladandayuthapani, V.;

Schellingerhout, D.; Cody, D. D. Invest. Radiol. 2015, 50 (1), 9-16.

(231) Mangla, S.; Choi, J. H.; Barone, F. C.; Novotney, C.; Libien, J.; Lin, E. BMC Res. Notes 2015, 8 (808), 1-6.

(232) Yamamoto, D.; Koizumi, H.; Ishima, D.; Kuroda, H.; Shibahara, I.; Niki, J.;

Miyasaka, K.; Watanabe, T.; Kondo, R.; Kumabe, T. Tohoku J. Exp. Med. 2019, 249 (3), 185-192.

(233) Geary, R. S.; Norris, D.; Yu, R.; Bennett, C. F. Adv. Drug Deliv. Rev. 2015, 87, 46-51.

(234) Perschbacher, K.; Smestad, J. A.; Peters, J. P.; Standiford, M. M.; Denic, A.; Wootla, B.; Warrington, A. E.; Rodriguez, M.; Maher, L. J. Nucleic Acid Ther. 2015, 25 (1), 11-19.

(235) Kovacevic, K. D.; Gilbert, J. C.; Jilma, B. Adv. Drug Deliv. Rev. 2018, 134, 36-50.

(236) Periayah MH; Halim AS; Saad AZM. Int. J. Hematol. Stem Cell Res. 2017, 11 (4), $319-327$.

(237) Korchinski, D. J.; Taha, M.; Yang, R.; Nathoo, N.; Dunn, J. F. Magn. Reson. Insights 2015, 8 (S1), 15-29.

(238) Chatterjee, K.; Sarkar, S.; Jagajjanani Rao, K.; Paria, S. Adv. Colloid Interface Sci. 2014, 209, 8-39.

(239) Thomas, R.; Park, I.; Jeong, Y. Y. Int. J. Mol. Sci. 2013, 14, 15910-15930.

(240) Gawande, M. B.; Goswami, A.; Asefa, T.; Guo, H.; Biradar, A. V.; Peng, D. L.; Zboril, R.; Varma, R. S. Chem. Soc. Rev. 2015, 44 (21), 7540-7590. 
(241) Smith, M.; McKeague, M.; DeRosa, M. C. MethodsX 2019, 6, 333-354.

(242) Janko, C.; Ratschker, T.; Nguyen, K.; Zschiesche, L.; Tietze, R.; Lyer, S.; Alexiou, C. Front. Oncol. 2019, 9 (FEB), 1-9.

(243) Azhdarzadeh, M.; Atyabi, F.; Saei, A. A.; Varnamkhasti, B. S.; Omidi, Y.; Fateh, M.; Ghavami, M.; Shanehsazzadeh, S.; Dinarvand, R. Colloids Surfaces B Biointerfaces 2016, 143, 224-232.

(244) Zhao, S.; Yu, X.; Qian, Y.; Chen, W.; Shen, J. Theranostics 2020, 10 (14), 62786309.

(245) Zamay, G. S.; Zamay, T. N.; Lukyanenko, K. A.; Kichkailo, A. S. Biomedicines 2020, $8(59), 1-14$.

(246) Guardia, P.; Labarta, A.; Batlle, X. J. Phys. Chem. C 2011, 115 (2), 390-396.

(247) Blasiak, B.; Van Veggel, F. C. J. M.; Tomanek, B. J. Nanomater. 2013, 2013, 112.

(248) Moraes Silva, S.; Tavallaie, R.; Sandiford, L.; Tilley, R. D.; Gooding, J. J. Chem. Commun. 2016, 52 (48), 7528-7540.

(249) Robinson, I.; Tung, L. D.; Maenosono, S.; Wälti, C.; Thanh, N. T. K. Nanoscale 2010, 2 (12), 2624-2630.

(250) Wang, L.; Luo, J.; Fan, Q.; Suzuki, M.; Suzuki, I. S.; Engelhard, M. H.; Lin, Y.; Kim, N.; Wang, J. Q.; Zhong, C. J. J. Phys. Chem. B 2005, 109 (46), 2159321601.

(251) Zhou, X. X.; Liu, J. F.; Jiang, G. Bin. Environ. Sci. Technol. 2017, 51 (7), 38923901.

(252) Mahan, M. M.; Doiron, A. L. J. Nanom 2018, 2018, 1-15. 
(253) Ciesienski, K. L.; Caravan, P. Curr. Cardiovasc. Imaging Rep. 2011, 4 (1), 77-84.

(254) Atanasova, I.; Sojoodi, M.; Leitaõ, H. S.; Shuvaev, S.; Geraldes, C. F. G. C.; Masia, R.; Guimaraes, A. S.; Tanabe, K. K.; Fuchs, B. C.; Caravan, P. Invest. Radiol. 2020, 55 (4), 209-216.

(255) Kumagai, M.; Sarmat, T. K.; Cabral, H.; Kaida, S.; Sekino, M.; Herlambang, N.; Osada, K.; Kano, M. R.; Nishiyama, N.; Kataoka, K. Enhanced in Vivo Magnetic Resonance Imaging of Tumors by PEGylated Iron-Oxide-Gold Core-Shell Nanoparticles with Prolonged Blood Circulation Properties. Macromolecular Rapid Communications. 2010, pp 1521-1528.

(256) Longmire, M.; Choyke, P. L.; Kobayashi, H. Nanomedicine 2008, 3 (5), 703-717. 